\title{
Assessment of Salmonids and Their Habitat Conditions in the Walla Walla River Basin
}

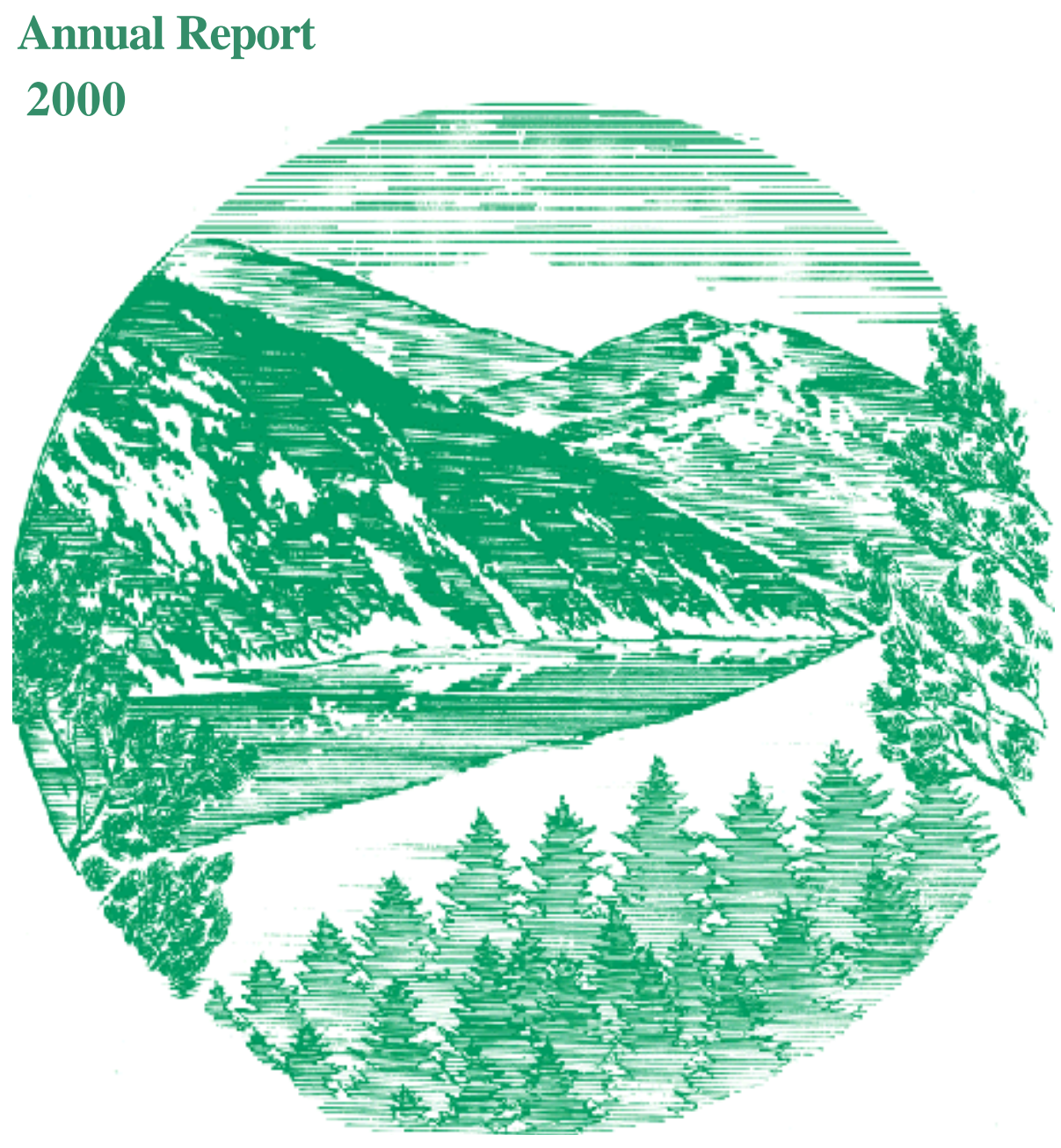

DOE/BP-00000485-1

November 2001 
This Document should be cited as follows:

Coyle, Terrence, David Karl, Glen Mendel, "Assessment of Salmonids and Their Habitat Conditions in the Walla Walla River Basin", Project No. 1998-02000, 116 electronic

pages, (BPA Report DOE/BP-00000485-1)

\author{
Bonneville Power Administration \\ P.O. Box 3621 \\ Portland, Oregon 97208
}

This report was funded by the Bonneville Power Administration (BPA), U.S. Department of Energy, as part of BPA's program to protect, mitigate, and enhance fish and wildlife affected by the development and operation of hydroelectric facilities on the Columbia River and its tributaries. The views in this report are the author's and do not necessarily represent the views of BPA. 


\section{Assessment of Salmonids and Their Habitat Conditions in the Walla Walla River Basin of Washington:}

\section{Annual Report}

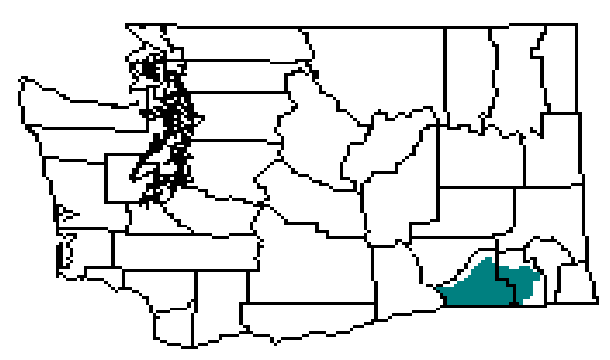

By

Glen Mendel, David Karl, \& Terrence Coyle Washington Department of Fish and Wildlife Fish Program - Fish Management Division 529 W. Main St. Dayton, WA 99328

\section{For}

\section{U.S. Department of Energy}

Bonneville Power Administration

Environment, Fish and Wildlife

P.O. Box 3621

Portland, OR 97208

Project Number 199802000

Contract Number 00000485-00001

November 2001 


\section{Acknowledgments}

Many people and organizations contributed to this project. First we would like to thank the Bonneville Power Administration for funding this project, and Charlie Craig (BPA) for his patience and assistance.

This project could not have progressed without the approval of landowners in granting access to their lands. We sincerely appreciate their cooperation and consideration.

We appreciate the assistance from the WDFW Snake River Lab. They shared equipment and provided us with some data and DNA samples from the Touchet River, as well as assisted with some electrofishing and spawning surveys. Other WDFW personnel also provided valuable assistance. Mike “Clem” Gembala, Arthur Curry, Kristin Lyonnais, Jeff McCowen, Lacey Gallaher, and Bart Hunking assisted with data collection and data entry.

Bill Neve and John Covert (DOE) provided valuable assistance both establishing and operating the constant recording stream flow monitors. Jon Germond (ODFW) and his staff collected DNA samples from steelhead and bull trout in the upper Walla Walla River. Thanks to W. S. "Wib" Wagoner for operating the adult steelhead trap on Yellowhawk Creek. Jim Shaklee was in charge of DNA analyses. We thank them all.

Joe Bumgarner, Bill Neve, and Craig Contour reviewed this draft and provided comments for revisions. We appreciate their assistance in preparation of this report. 


\section{Table of Contents}

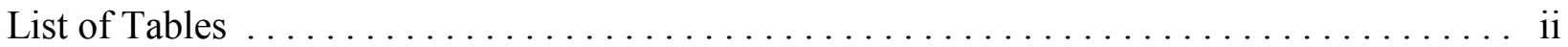

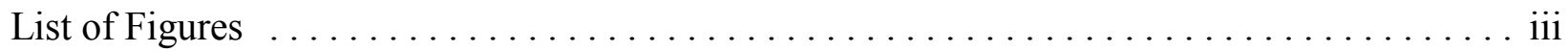

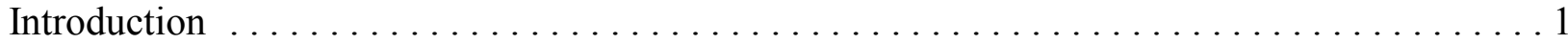

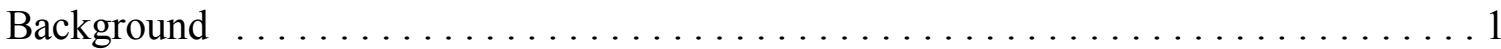

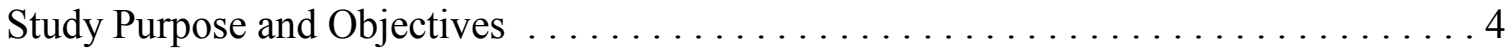

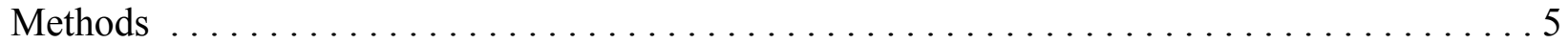

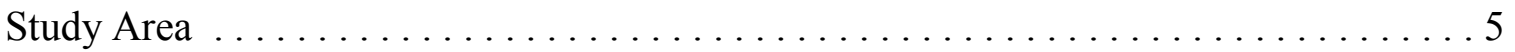

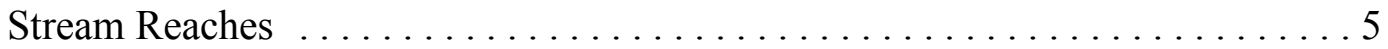

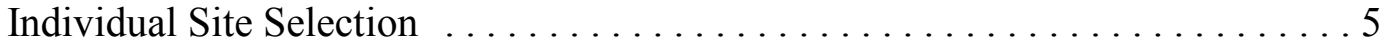

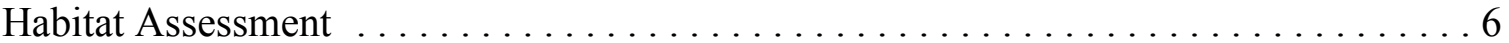

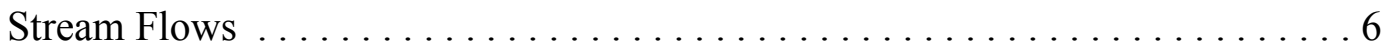

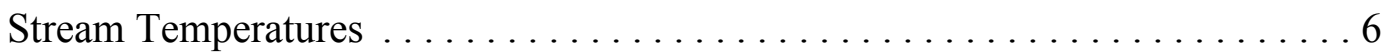

Water Quality .................................... 9

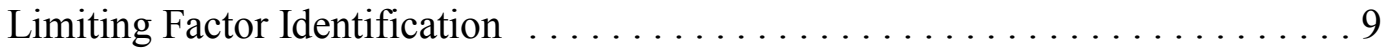

Fish Stock Assessment .................................. 10

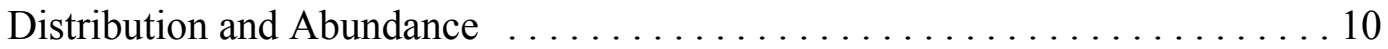

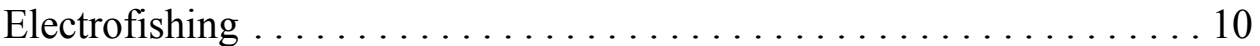

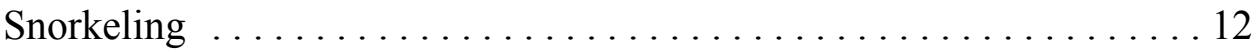

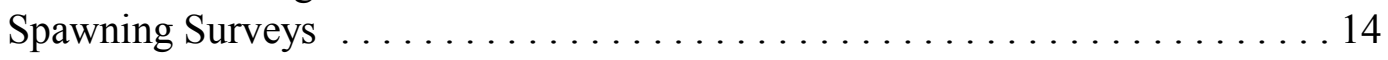

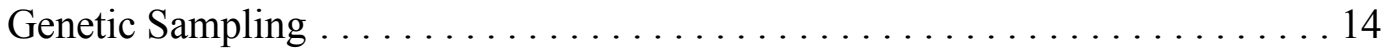

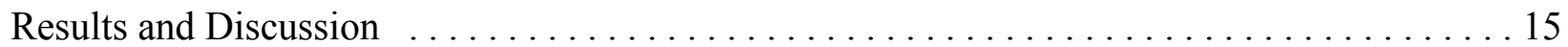

Habitat Assessment . . . . . . . . . . . . . . . . . . . . . . . . . . . 15

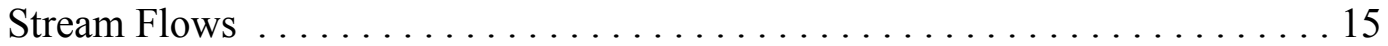

Stream Temperatures ................................ 19

Water Quality ....................................... 19

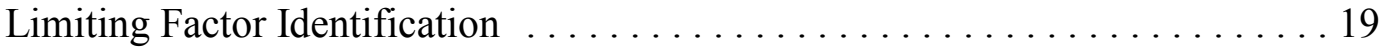

Fish Stock Assessment ..................................... 20

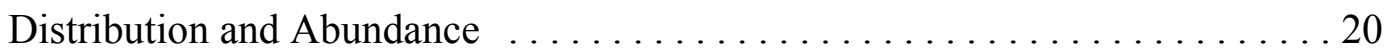

Electrofishing .................................... 31

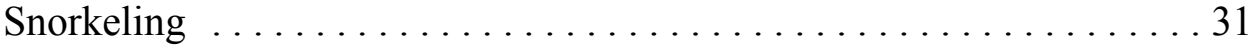

Non-Salmonid Species Abundance and Distribution ............. 31

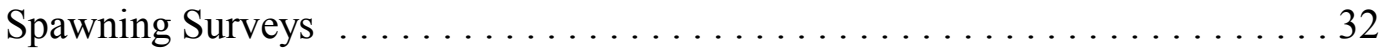

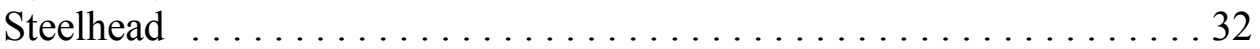

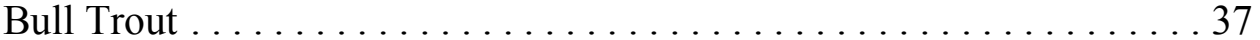

Genetic Sampling ................................... 39

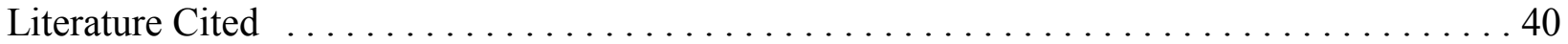




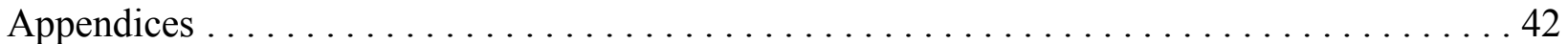

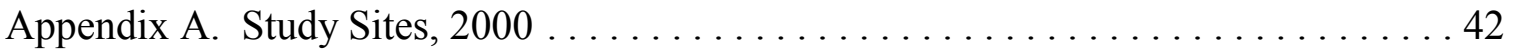

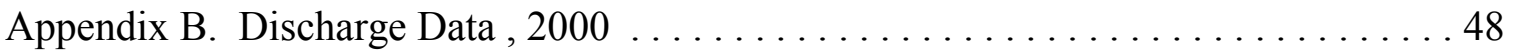

Appendix C - Stream Temperature Graphs (EF), $2000 \ldots \ldots \ldots \ldots \ldots \ldots \ldots \ldots \ldots \ldots$

Appendix C1 - Additional Stream Temperature Graphs (EF), 2000 . . . . . . . . 74

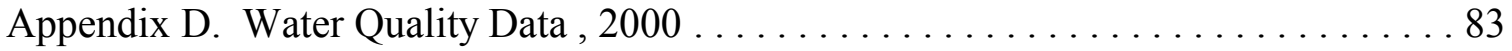

Appendix E. Qualitative Electrofishing, $2000 \ldots \ldots \ldots \ldots \ldots \ldots \ldots$. . . . . . 87

Appendix F. Relative Abundance of Non-Salmonids, 2000 . . . . . . . . . . . 96

Appendix G. Preliminary Results of Instream Flow Incremental

Methodology for the Walla Walla River and Mill Creek, 1999 . . . . . . . . . . . 99 


\section{List of Tables}

Table 1. Categories of relative abundance (per site) for non-salmonids . . . . . . . . . 12

Table 2. Densities of salmonids from electrofishing sites in the Touchet River and some of its tributaries, summer and fall, $2000 \ldots \ldots \ldots \ldots \ldots \ldots \ldots \ldots \ldots \ldots$

Table 3. Densities of salmonids from electrofishing sites in the Walla Walla River

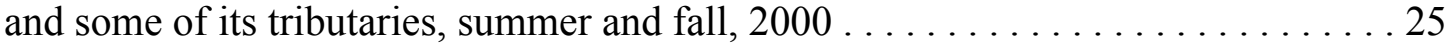

Table 4. Biomass of salmonids from electrofishing sites in the Touchet River and some of its tributaries, summer and fall, $2000 \ldots \ldots \ldots \ldots \ldots \ldots \ldots$

Table 5. Biomass of salmonids from electrofishing sites in the Walla Walla River and some of its tributaries, summer and fall, $2000 \ldots \ldots \ldots \ldots \ldots \ldots \ldots \ldots \ldots$

Table 6. Densities of salmonids from snorkel surveys in the Touchet River and Walla Walla River, summer and fall, $2000 \ldots \ldots \ldots \ldots \ldots \ldots \ldots \ldots$

Table 7. Steelhead spawning survey summary for some of the tributaries of The Walla Walla River in Washington State, 2000 . . . . . . . . . . . . . . . . 32

Table 8. Bull trout spawning survey summary for the Wolf Fork of the

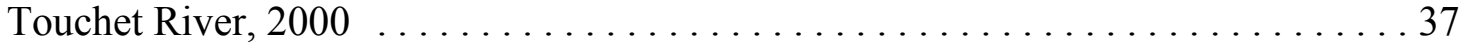

Table 9. Bull trout spawning survey summary for the Burnt Fork of the

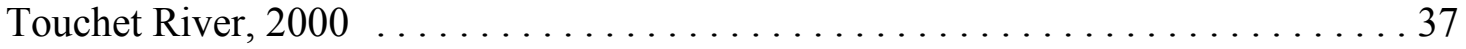




\section{List of Figures}

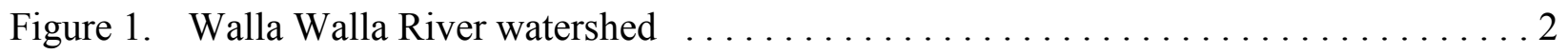

Figure 2. Relative locations of flow monitoring sites in the Walla Walla Basin, 2000 . . . . 7

Figure 3. Relative locations of temperature sites in the Walla Walla Basin, $2000 \ldots \ldots \ldots$. . 8

Figure 4. Relative locations of quantitative electrofishing and snorkeling sites in the Walla Walla Basin, 2000 .............................. 11

Figure 5. Relative locations of qualitative electrofishing sites in the WallaWalla

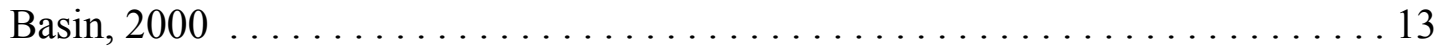

Figure 6. Walla Walla River stream discharge (CFS) and daily maximum water temperatures (EF) every four hours, above Pepper Bridge, $2000 \ldots \ldots \ldots \ldots \ldots$

Figure 7. Yellowhawk Creek stream discharge (CFS) and daily maximum water temperatures (EF) every four hours, near confluence with the Walla Walla River, $2000 \ldots \ldots 16$

Figure 8. Walla Walla River stream discharge (CFS) and daily maximum water temperatures (EF) every four hours, below Mojonnier Rd and Burlingame Dam, 2000 . . . . . 17

Figure 9. Walla Walla River stream discharge (CFS) and daily maximum water temperatures (EF)every four hours, above Detour Road, $2000 \ldots \ldots \ldots \ldots \ldots \ldots \ldots \ldots \ldots$

Figure 10. Touchet River stream discharge (CFS) and daily maximum water temperatures (EF) every four hours, below Sims Road Bridge, $2000 \ldots \ldots \ldots \ldots \ldots \ldots \ldots \ldots$

Figure 11. Touchet River stream discharge (CFS) and daily maximum water temperatures (EF) every four hours, above Cummins Rd Bridge, $2000 \ldots \ldots \ldots \ldots \ldots \ldots \ldots$

Figure 12. Bull trout spawning survey summary for the Wolf Fork of the Touchet

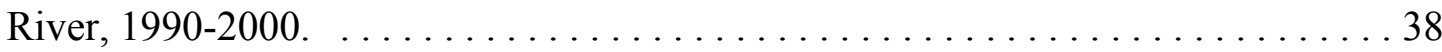

Figure 13. Bull trout spawning survey summary for the North Fork of the Touchet River, 1994-2000. 


\section{Introduction}

Concerns about the decline of native salmon and trout populations have increased among natural resource managers and the public in recent years. As a result, a multitude of initiatives have been implemented at the local, state, and federal government levels. These initiatives include management plans and actions intended to protect and restore salmonid fishes and their habitats.

In 1998 bull trout were listed under the Endangered Species Act (ESA), as "Threatened", for the Walla Walla River and its tributaries. Steelhead were listed as "Threatened" in 1999 for the mid-Columbia River and its tributaries. These ESA listings emphasize the need for information about the threatened salmonid populations and their habitats.

The Washington Department of Fish and Wildlife (WDFW) is entrusted with "the preservation, protection, and perpetuation of fish and wildlife....[and to] maximize public recreational or commercial opportunities without impairing the supply of fish and wildlife (WAC 77.12.010)." In consideration of this mandate, the WDFW submitted a proposal in December 1997 to the Bonneville Power Administration (BPA) for a study to assess salmonid distribution, relative abundance, genetics, and the condition of their habitats in the Walla Walla River basin.

The primary purposes of this project are to collect baseline biological and habitat data, to identify major data gaps, and to draw conclusions whenever possible. The study reported herein details the findings of the 2000 field season (March to November, 2000).

\section{Background}

The Walla Walla River and its major tributaries, including the Touchet River, comprise a watershed of 1,758 square miles (ACOE 1997) and 2,454 major stream miles (Knutson et al. 1992). The majority of the watershed (73\%) lies within Washington State, with the remainder in Oregon (Figure 1). The Walla Walla River originates from a fine network of deeply incised streams on the western slopes of the Blue Mountains. The Touchet River originates from similar streams on the northwestern slopes of the Blue Mountains, and also from seasonal streams draining Palouse hillsides to the north. The Walla Walla River drains into the Columbia River near Wallula Gap, about 21 miles above McNary Dam and 6 miles above the Oregon border. The Touchet River drains into the Walla Walla River just west of the town of Touchet, WA.

Historic and contemporary land-use practices have had a profound impact on the salmonid species abundance and distribution in the watershed. Fish habitat in area streams has been severely degraded by urban and agricultural development, grazing, tilling, logging, recreational activities, and flood control structures. Agricultural diversions have severely impacted stream flows in the Walla Walla River since the 1880s (Nielson 1950). Nearly all (99\%) of the surface water diversions within Washington are for the purpose of irrigation (Pacific Groundwater Group 


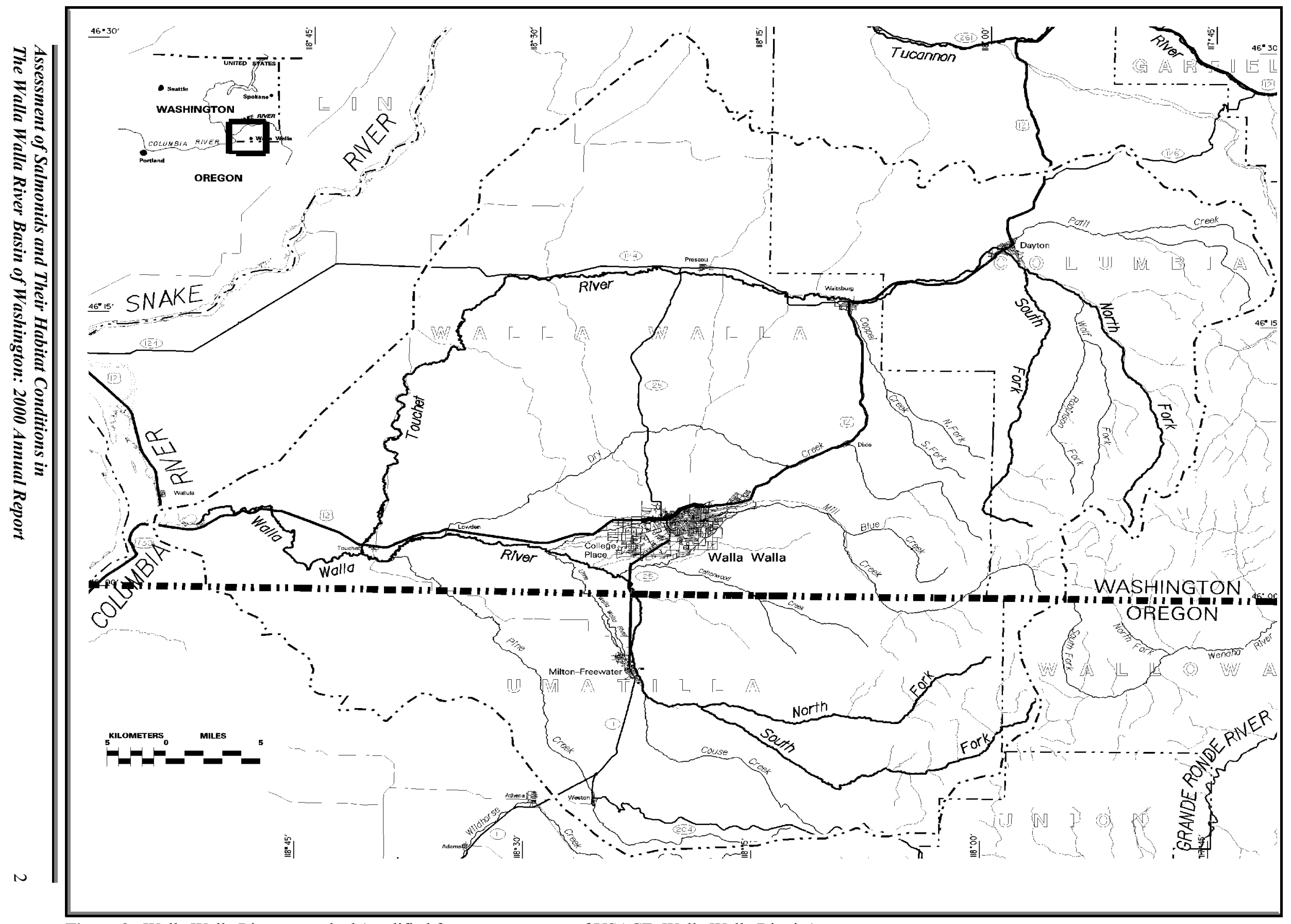

Figure 2. Walla Walla River watershed (modified from map courtesy of USACE, Walla Walla District). 
1995). The reduced stream flows created by irrigation withdrawals adversely impact salmonid survival within the basin. Additionally, many unscreened or partly screened diversions and fish passage barriers exist within the basin.

Out-of-basin impacts to local fish populations have been substantial. Salmon migrating to or from the ocean must pass through four dams and reservoirs on the Columbia River before reaching their destination. Juvenile and adult salmonid mortalities occur as they pass through each reservoir or dam. Other impacts include over-harvest, habitat destruction in the lower Columbia River and estuaries, predation, and industrial pollution.

Historically the basin probably produced substantial runs of both spring chinook and summer steelhead. The last substantial run of wild chinook took place in 1925; thereafter chinook populations continued a precipitous decline, and the species is considered extirpated in the basin (Nielson 1950, ACOE 1997). Anecdotal accounts and reports of historic fisheries in adjacent basins, indicate that chum and coho could have occurred in substantial numbers in the Walla Walla Basin (Pirtle 1957), but little written documentation exists. Endemic steelhead persist throughout much of the basin, but the population is considered depressed (WDF and WDW 1993). Annually, approximately 200,000 non-endemic hatchery steelhead (Lyons Ferry stock) are released in the middle Touchet and lower Walla Walla rivers under the Lower Snake River Compensation Program (LSRCP) to provide harvest mitigation for the four lower Snake River dams.

Not all native salmonids in the basin are anadromous. Whitefish, bull trout and rainbow/redband trout exist within the basin. However, only rainbow/redband trout retain a wide distribution. In the past, bull trout are thought to have been widely distributed in the basin. Currently, bull trout distribution is generally limited to montane upper tributaries of the Touchet River, Walla Walla River, and Mill Creek (Mongillo 1993). However, bull trout are known to migrate into the middle or lower reaches of these rivers during the winter months. Many factors have led to the decline of bull trout in southeast Washington. Damaged riparian vegetation, increased sedimentation, and decreased water flows have resulted in elevated water temperatures beyond the tolerance of this cold water species (Mongillo,1993). Introduced rainbow trout or brown trout may have increased competition or predation for bull trout.

Several non-native fish species have been introduced to support recreational fishing, or they have strayed into the basin. The Washington Department of Game (now WDFW) began stocking brown trout (Salmo trutta) in the Touchet River in the July, 1965. Stocking was discontinued in 1999 due to concerns about competition, hybridization, and predation with native bull trout, steelhead, or rainbow/redband trout. Carp were introduced as early as 1884 (Walla Walla Daily Journal 1884). Channel catfish, smallmouth bass, and bluegill are some of the warm water fish that now occur in the lower basin. Additionally, in 1999, three-spine stickleback (Gasterosteus aculeatus) were found in the Walla Walla river by WDFW personnel involved with this project. 


\section{Study Purpose and Objectives}

The purpose of this study is to determine fish passage, rearing, and spawning conditions for steelhead and for potential reintroduction of chinook salmon, and to assess steelhead and bull trout distribution, densities, habitat, and genetic composition in the Walla Walla watershed.

Specific objectives and tasks were outlined in WDFW's proposal and statement of work to the Bonneville Power Administration (BPA Project \# 199802000). Some tasks had to be scaled back or postponed. Multi-year study objectives include:

1. Assess baseline habitat conditions for salmonids in the Washington portion of the Walla Walla watershed;

2. Determine salmonid distribution and relative abundance in the Washington portion of the Walla Walla watershed; and

3. Identify genetic stocks of steelhead and bull trout in the Walla Walla watershed.

\section{Specific objectives and tasks were outlined in the statement of work. Tasks included:}

- Establish constant recording temperature and flow data loggers in the Walla Walla River basin, to identify available water for salmon passage and rearing, as well as temperature limitations for salmonid passage, spawning and rearing;

- Conduct an Instream Flow Incremental Methodology (IFIM) study, in order to quantify available habitat as it relates to stream discharge (flow);

- Conduct biweekly manual stream flow and temperature measurements to calibrate instream monitor data outputs, and to provide data for reaches that did not have instream discharge monitors;

- Monitor water quality by sampling dissolved oxygen, $\mathrm{pH}$, turbidity, and conductivity;

- Conduct electrofishing to determine salmonid distribution, abundance, and habitat use;

- Conduct snorkel surveys during the spring and summer to supplement electrofishing data and for seasonal density comparisons;

- Conduct periodic flights of the lower Walla Walla and Touchet Rivers to determine continuity of stream flows for adequate fish passage and rearing;

- Conduct general habitat surveys in portions of the stream with potential for salmonid use to quantify habitat conditions and identify limiting factors (This task has been deferred).

- Conduct steelhead and bull trout spawning surveys to determine spawning timing and distribution, and to establish an index of relative abundance.

- Collect tissue samples from bull trout and steelhead for genetic analyses. 


\section{Study Area}

The study area encompasses the greater Walla Walla River basin in Washington State (Figure 1). The Walla Walla River, the Touchet River, and Mill Creek are the major rivers within the basin. The Walla Walla River in Washington, lower Mill Creek, and the Touchet River and thier tributaries, were the primary study reaches in 2000 .

\section{Stream Reaches}

Representative stream reaches were identified based on general physical characteristics and readily identifiable landmarks (Appendix A). General physical characteristics included: slope, width, depth, and temperature; as well as, predominant adjacent land uses. Landmarks included towns, roads, and bridges.

\section{Individual Site Selection}

Most of the study streams are in private ownership, therefore it was necessary to obtain permission from landowners to access potential sites. Owners of property bordering the study streams were identified from county assessment records and contacted in person or by telephone. For convenience, public land was utilized whenever possible. Study sites were distributed to comprehensively cover the study area (Appendix A), and sites are listed and identified in order from upstream to downstream.

River miles were determined by measuring 1:24000 USGS topographic maps with a map wheel. River miles were determined by measuring the distance between the confluence of each stream and the study site. These locations should be considered approximate due to the limited precision of this method.

Electrofishing sites were selected randomly from access areas. Selections of top and bottom net locations were also randomized. Site lengths sometimes had to be modified to avoid unsuitable stream features, such as deep pools, rapids, multiple channels, and/or for safety concerns.

Snorkeling sites were designed to extend and compliment the area initially surveyed by electrofishing. Sites were located using the same randomization process used for establishing electrofishing sites. 


\section{Habitat Assessment}

\section{Stream Flows}

Stream discharge was measured using two methods. Manual flow measurements were taken at selected sites according to standard techniques (Armour and Platts 1983) using a Swoffer model 2100 flow meter. Discharge was calculated in cubic feet per second (cfs) with Quattro Pro $(\mathrm{C}$ spreadsheets. The second method involved the use of continuous flow data loggers (Unidata America, Model KB/DSP 128K). The monitors were placed at three sites on the Walla Walla River, and two sites on the lower Touchet River (Appendix A, Figure 2). WDFW contracted with the Washington Department of Ecology (WDOE) to maintain the monitors and collect the data. Manual flow measurements were taken approximately every two weeks by WDFW near each of the flow monitors to correlate the discharge and stage readings recorded by the monitors. An index site was a location where discharge measurements were taken approximately every two weeks, compared to periodic flow sites which were flows taken just occasionally (Appendix A, Figure 2).

IFIM - We subcontracted with Hal Beecher (WDFW) and Brad Caldwell (WDOE) to conduct an IFIM study on the Walla Walla River and lower Mill Creek in 1999. Preliminary results are provided in Appendix G.

\section{Stream Temperatures}

We used three methods to collect water temperatures. Manual water temperature $(E F)$ was measured at each site using standard field thermometers. The second method involved the use of temperature data loggers (Onset Corporation, Optic StowAway, or TidbiT Temp Data Logger ${ }^{\circledR}$ ), which were set to continuously measure temperatures in $\mathrm{EF}$ at 30 minute intervals. The monitors were placed at sites throughout the Walla Walla River basin (Appendix A, Figure 3). WDFW maintained the temperature monitors and downloaded the data using an Optic Stowaway Shuttle ${ }^{\circledR}$. Temperature data were exported from Onset Boxcar 4.0 software into Quattro Pro spreadsheets. Daily minimum, maximum, and mean temperatures were prepared using a Quattro Pro spreadsheet macro.

The third method involved the use of continuous flow and temperature data loggers (Unidata America, Model KB/DSP 128K). The monitors collect both stream discharge (stage value) and temperature data every 15 seconds and stores the data every four hours as averages for discharge and minimum, maximum, and mean temperatures. The monitors were used to collect temperatures as a substitute for the stowaway temperature loggers at their respective sites (Appendix A, Figure 3). The accuracy of field thermometers and data loggers was evaluated using a laboratory calibrated thermometer (Kessler Instruments).

Stream temperatures were also collected by the Army Corp of Engineers using temperature loggers provided by the Walla Walla Conservation District (Appendix C1, Figure 3). 


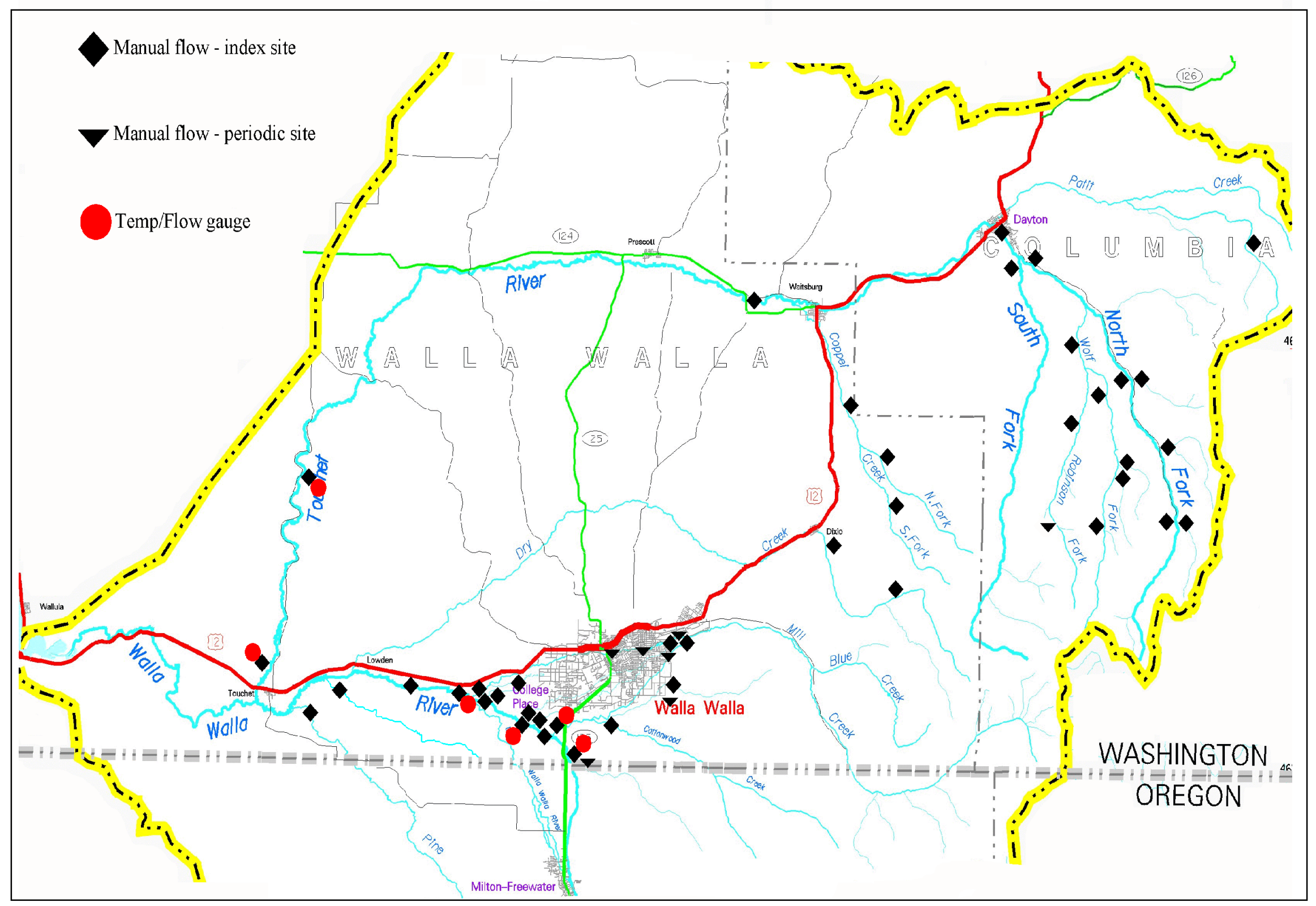

Figure 2. Relative locations of flow monitoring sites in the Walla Walla Basin, 2000. 


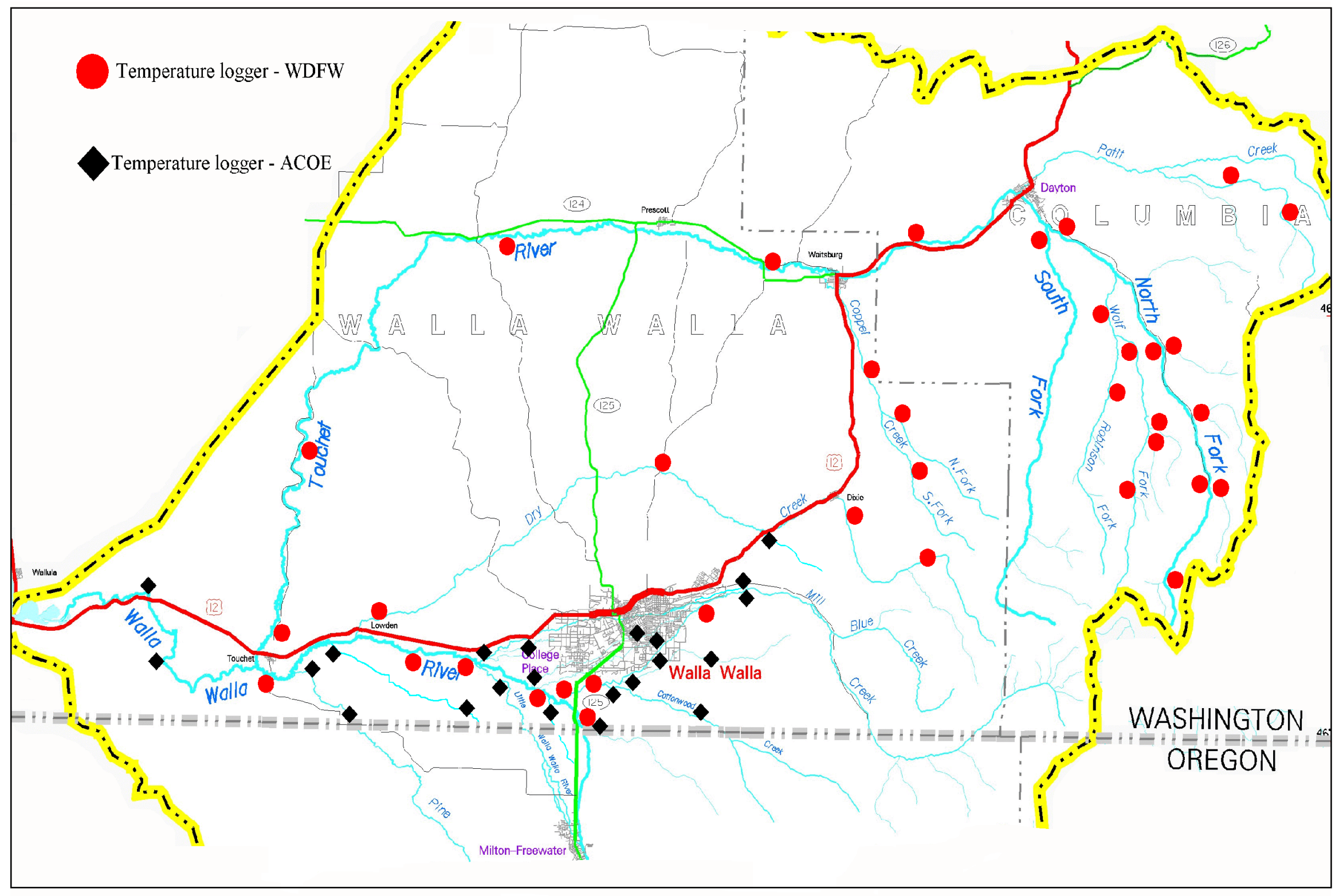

Figure 3. Relative locations of temperature logger sites in the Walla Walla Basin, 2000. 


\section{Water Quality}

Miscellaneous water quality data were collected by WDFW during the 2000 field season. Sampled water quality variables included conductivity (umhos/ $\mathrm{cm}$ ), and $\mathrm{pH}$ (Appendix D). Conductivity data was collected using an Orion Model 115 conductivity meter. We collected $\mathrm{pH}$ data using a VWR brand model $2000 \mathrm{pH}$ meter. Meters were calibrated according to manufacturer specifications prior to collection efforts.

\section{Limiting Factor Identification}

One of the study goals was to identify and document physical barriers to salmonid passage, spawning and rearing. Field personnel noted the presence of potential barriers and provided the information to local biologists to coordinate habitat rehabilitation efforts. The activity of two major irrigation diversion structures, Hofer Dam on the Touchet River, and Burlingame Diversion on the Walla Walla River, were also noted throughout the season.

Physiological barriers to salmonid passage and survival, in the form of excessive temperatures, inadequate flows, and degraded habitat were also identified by examining tables and graphs of data collected by instream monitors and manual sampling. Maximum temperatures, as well as the number of days with temperatures exceeding $75 \mathrm{EF}$ (lethal to salmonids if prolonged), and presence or absence of salmonid fishes at study sites, were factors taken into consideration. 


\section{Fish Stock Assessment}

\section{Distribution and Abundance}

\section{Electrofishing}

A Smith-Root Model 11A or 12B electrofishing backpack unit was used to collect fish so we could calculate densities at various study sites in the Walla Walla basin (Figures 3, 4). We used pulsed DC (direct current) between 400 and 600 volts. Sites were delimited by block nets spanning the channel, usually placed approximately 30 meters apart. Block nets prevented fish from entering or leaving the site, so that densities could be calculated (Platts et al. 1983). The operator began at the upstream net and worked downstream, covering the entire wetted width. One "pass" was completed when the downstream net was reached. All sites received at least two sequential passes. A $60 \%$ reduction was required between the first and second passes for each salmonid species and estimated age class. If the $60 \%$ reduction was not met, a third pass was conducted. Stunned fish were collected with dip nets and placed in buckets until they could be sampled for lengths and weights. Collected fish were

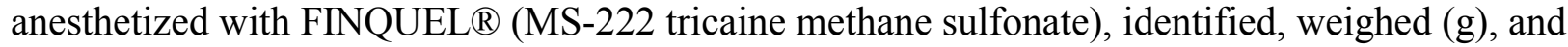
measured to fork length $(\mathrm{mm})$.

Fork lengths collected during quantitative electrofishing were used to create length frequency histograms. The histograms were used to determine age classes (Mendel et al. 1999). These age class delineations were checked against ages determined from reading fish scales that were collected from several of the stream reaches. Age class groupings were specific for each stream reach.

A removal-depletion software program developed by the U.S. Forest Service (Van Deventer and Platts, 1983) was used to calculate population densities $\left(\# / 100 \mathrm{~m}^{2}\right)$ for each salmonid species, by age class. The average weight (grams) of each age class was multiplied by its density to calculate biomass $\left(\mathrm{g} / 100 \mathrm{~m}^{2}\right)$ per age class.

Area sampled was determined by multiplying site length by the average of four or more site width measurements. A brief description of the riparian, bank stability, substrate, pools/riffle ratio, and the presence of large organic debris ( LOD) was recorded for each site.

Fish identification included genus and species for all Salmonids, and most Cottids, and Cyprinidae; and genus only for Catostomidae, and Petromyzontidae. Our sampling protocol was to collect 10-20 of each non-salmonid species at each site. Non-salmonid species were assigned a relative abundance ranking value based on general observations made during electrofishing (Table 1). 


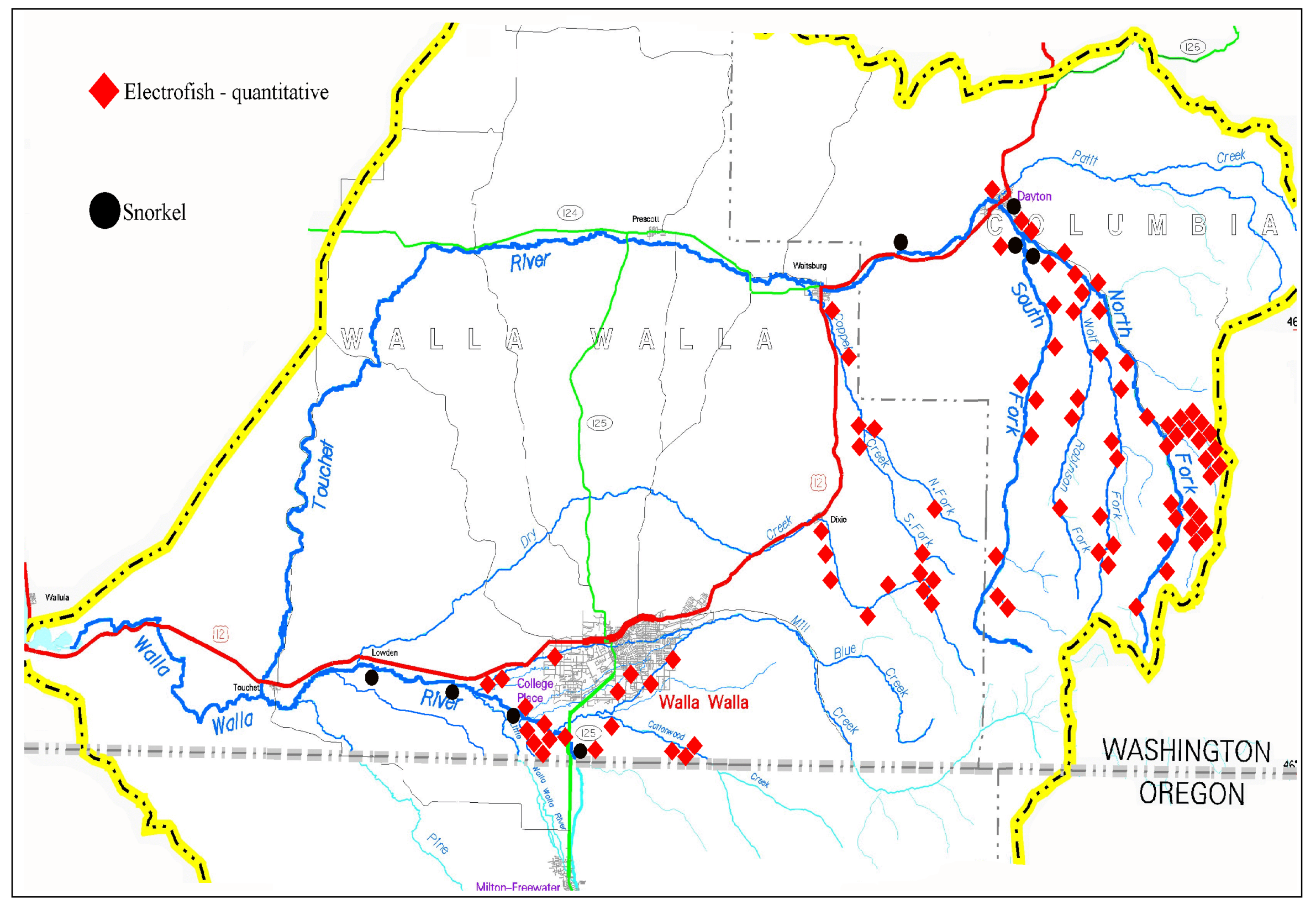

Figure 4. Relative locations of quantitative electrofishing and snorkeling sites in the Walla Walla Basin, 2000. 
Relative abundance for non-salmonid species were treated semi-quantitatively. For each species in each site, a relative abundance was determined. The relative abundance was assigned a corresponding ranking value (Table 1). Ranked values were averaged to determine a relative abundance for each species per designated reach. Relative abundance data were tabulated to provide qualitative comparisons between reaches and species.

\begin{tabular}{|lcc|}
\hline Table 1. Categories of relative abundance (per site) for non-salmonids. & \\
\hline Category & Count (individuals seen) & Ranking Value \\
\hline Absent & 0 & 0 \\
Rare & $1-3$ & 1 \\
Uncommon & $4-10$ & 2 \\
Common & $11-100$ & 3 \\
Abundant & $100+$ & 4 \\
\hline
\end{tabular}

We also conducted "qualitative" electrofishing surveys in several Walla Walla and Touchet River tributaries (Figure 4). These surveys enabled us to cover large areas relatively quickly as they did not entail the use of block nets or repeat passes with the electrofisher. We electrofished while moving upstream and capturing fish to determine species presence, size of fish (age class), and their relative abundance. We also noted the presence or general abundance of non-salmonids. This method supplemented our intensive "quantitative" electrofishing surveys and our snorkel surveys to provide a more complete view of salmonid distribution and abundance.

\section{Snorkeling}

Snorkeling sites were generally 90-120 meters in length. Snorkelers moved upstream, counting and identifying species, and estimating the age class of all salmonid fishes. Counts were recorded on PVC armbands. General abundance of non-salmonids were also noted. Snorkel surveys could be performed in deeper water, braided channels, and at other locations where electrofishing was not feasible or effective. Another advantage of snorkeling was that we were able to cover a large stream area in a short period of time and still provide density estimates. Snorkel surveys were conducted at selected sites both in the spring and summer for comparisons of salmonid distribution and densities temporally and by geographic location (Figure 3).

Salmonids observed during snorkel surveys were classified by age class based on their estimated size. Snorkelers reported genus classifications for all non-salmonid fish. Age class and relative abundance of non-salmonids were estimated and recorded. Site length and width measurements were taken to calculate the area surveyed. Brief habitat descriptions were recorded . 


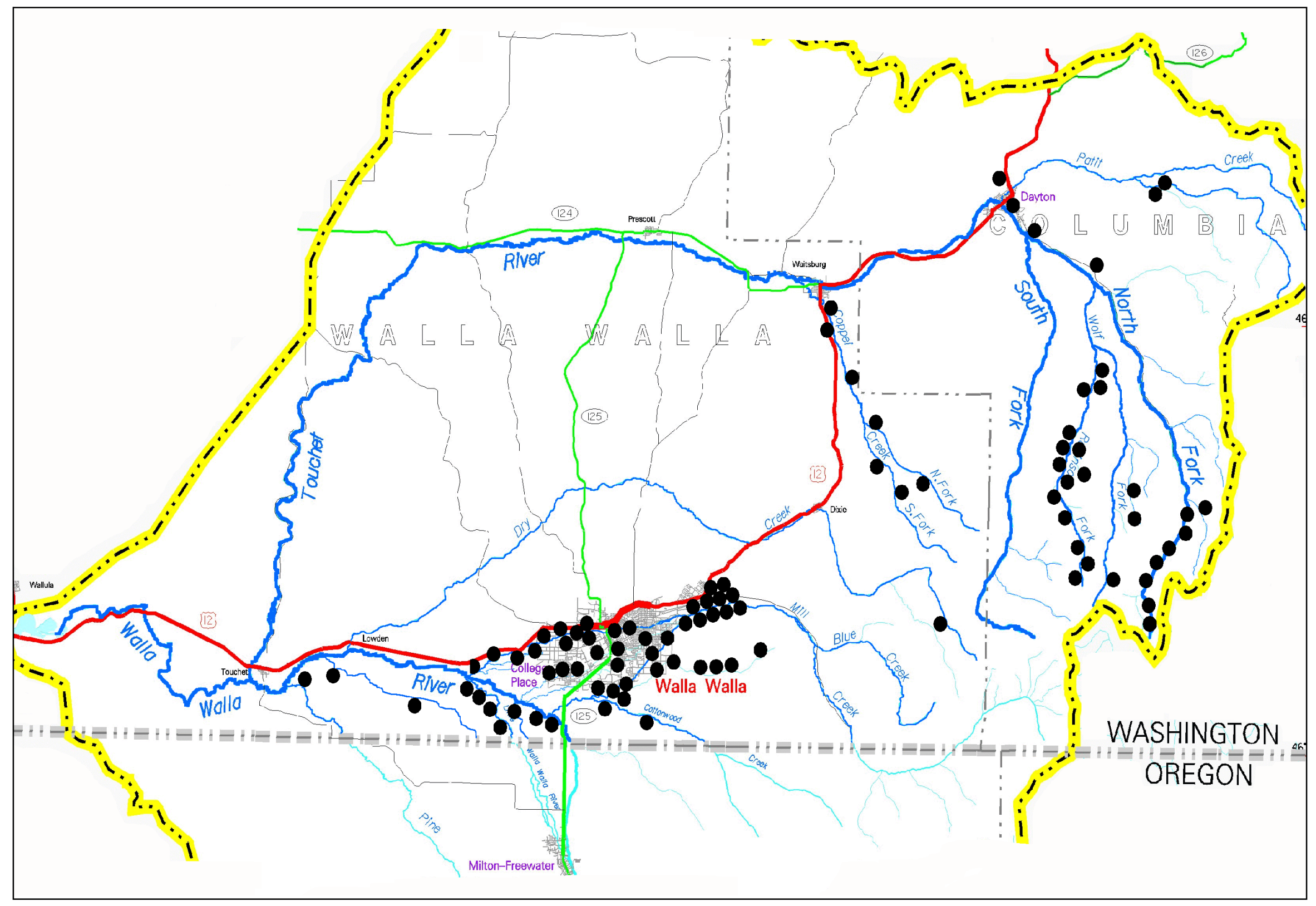

Figure 5. Relative locations of qualitative electrofishing sites in the Walla Walla Basin, 2000. 


\section{Spawning Surveys}

Spawning surveys were conducted in the same manner for both steelhead and bull trout. Surveyors walked downstream and visually identified spawning fish and/or redds (nests). Redds were easily identified, characterized by an area of clean gravel with a large depression and mound. Each redd observed was assigned a two-part identification code representing the survey number and the redd number. A flag was hung in adjacent vegetation, and marked with the identification (ID) code, the date, and the surveyor's initials, so the same redd would not be counted in subsequent surveys. Each redd was recorded in a notebook with the date, time, ID code, general description of the redd and its location. Counts were tallied for each designated stream reach.

\section{Genetic Sampling}

Sampling of salmonid tissues was undertaken by WDFW, cooperating agencies, and volunteer personnel for later genetic analyses. Fin clips or opercle punches were obtained from adult steelhead, rainbow trout/steelhead, bull trout, and whitefish. Tissue was collected at established fish traps on the Walla Walla River, Touchet River, Mill Creek, and Yellowhawk Creek and during electrofishing efforts within the basin. Fin clips were placed in tubes of $95 \%$ ethanol for preservation, labeled and transported to the WDFW Genetics Stock Identification Lab in Olympia. Fin clips provide sufficient DNA material for genetic analysis, without killing the fish (Olsen et al. 1996). A non-lethal method of genetic sampling was preferred due to the current ESA listings for bull trout and wild steelhead in the Walla Walla River basin. 


\section{Results and Discussion}

\section{Habitat Assessment}

\section{Stream Flows}

Stream flows in the Walla Walla River basin follow a fundamental pattern initiated by a rapid decline in discharge in late June, followed by low summer flows and increased discharge in the fall and winter. However, sites in proximity to major irrigation facilities exhibited more erratic stream flow patterns. Irrigation withdrawals included pumps, "push-up"dams for gravity diversions and irrigation district dams and canals. The reduced flows represent the end of the spring runoff, water diversions for agricultural irrigation, and the lack of summer precipitation in the basin. The recharge in the fall is generated because of fall precipitation and after most water diversions are discontinued or reduced.

In 2000, irrigators, under a settlement agreement with the USFWS, maintained a minimum instream flow of 13 cfs at Nursery Bridge in Milton Freewater, OR. Nursery Bridge is approximately four miles upstream from the Washington state line. The additional water made an immediate impact in Oregon by considerably reducing the historic dewatered area. A continuous flow monitor placed at the Washington state line showed a slight increase in flow, conceivably caused by additional groundwater recharge. Unfortunately, we had multiple problems with the flow monitor at the state line, therefore the data is inconclusive. Also, under the auspices of the settlement agreement, Touchet Gardenia Co., in Washington maintained at least a $10 \mathrm{cfs}$ streamflow past Burlingame Dam during their spring, early summer, and fall irrigation season.

Reduced flows downstream of major irrigation diversions were observed during the field season (Appendix B). Specific observations included: (1) dewatering of the Walla Walla River channel for about 0.5 miles around Tum-a-lum Bridge, in Oregon, with some recharge near the Oregon State line; (2) sharp flow reductions below Burlingame Diversion in mid to late June and again in mid-October through mid- November (as recorded by manual and instream flow monitors); and (3) a steep decline in flows on the Touchet River below Hofer Diversion in August (as recorded by manual and instream flow monitors).

The 1999-2000 winter provided an average snowpack in the Blue mountains. Spring/summer discharges for the Touchet River were generally lower for the same sites in 2000 than they were in 1999. In the Blue mountains, the Touchet station recorded a maximum snow water equivalent of 36.1 inches March and April 2000. Comparatively, in 1999 the maximum snow water equivalent recorded for March and April were 55.9 and 56.8 (WA NRCS website ftp://ftp.wcc.nrcs.usda.gov). 


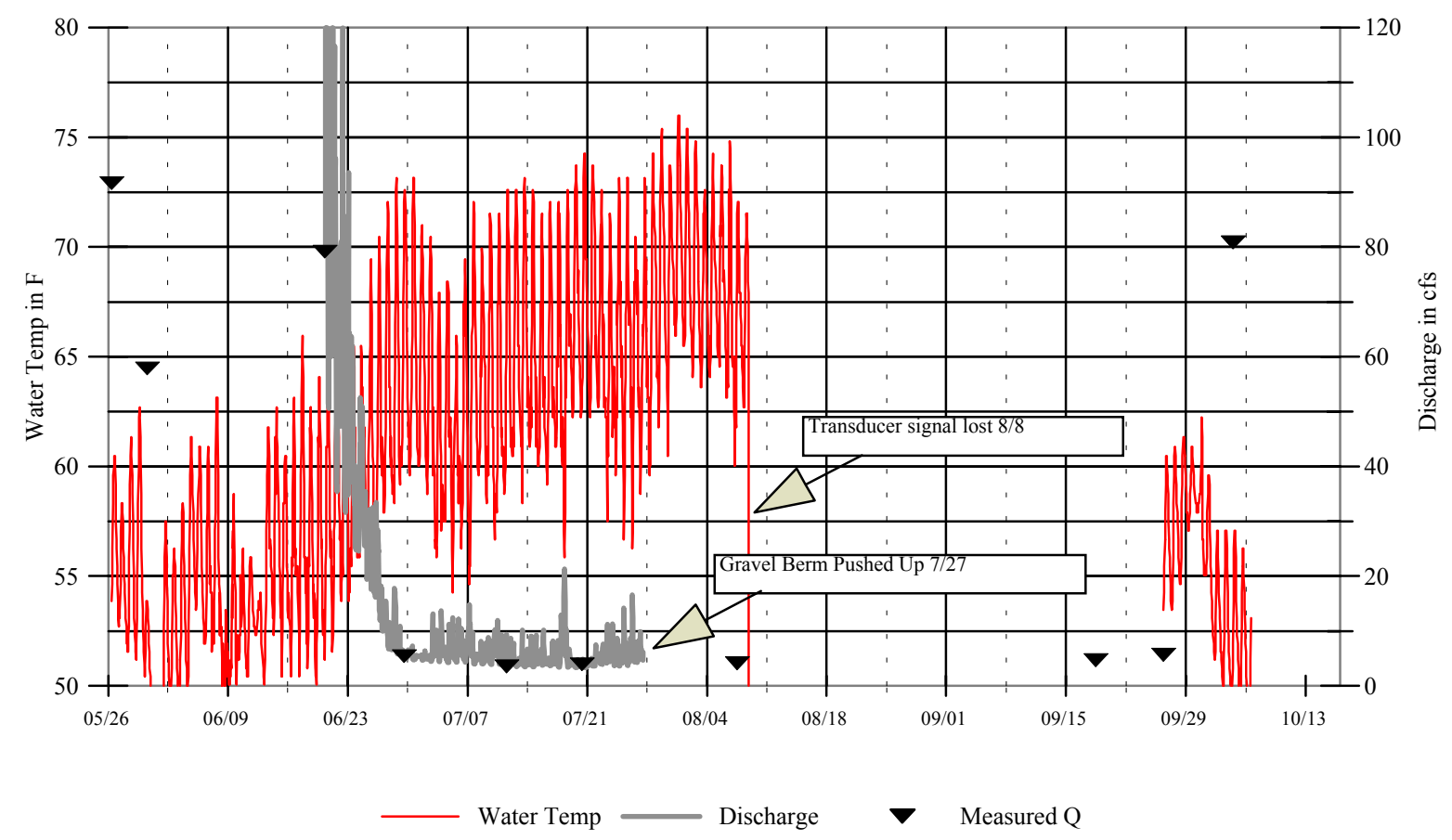

Figure 6. Walla Walla River stream discharge (CFS) and daily maximum water temperatures (EF) every four hours, above Pepper Rd Bridge(WW-2), 2000. (Field Q = manual stream discharge measurement)

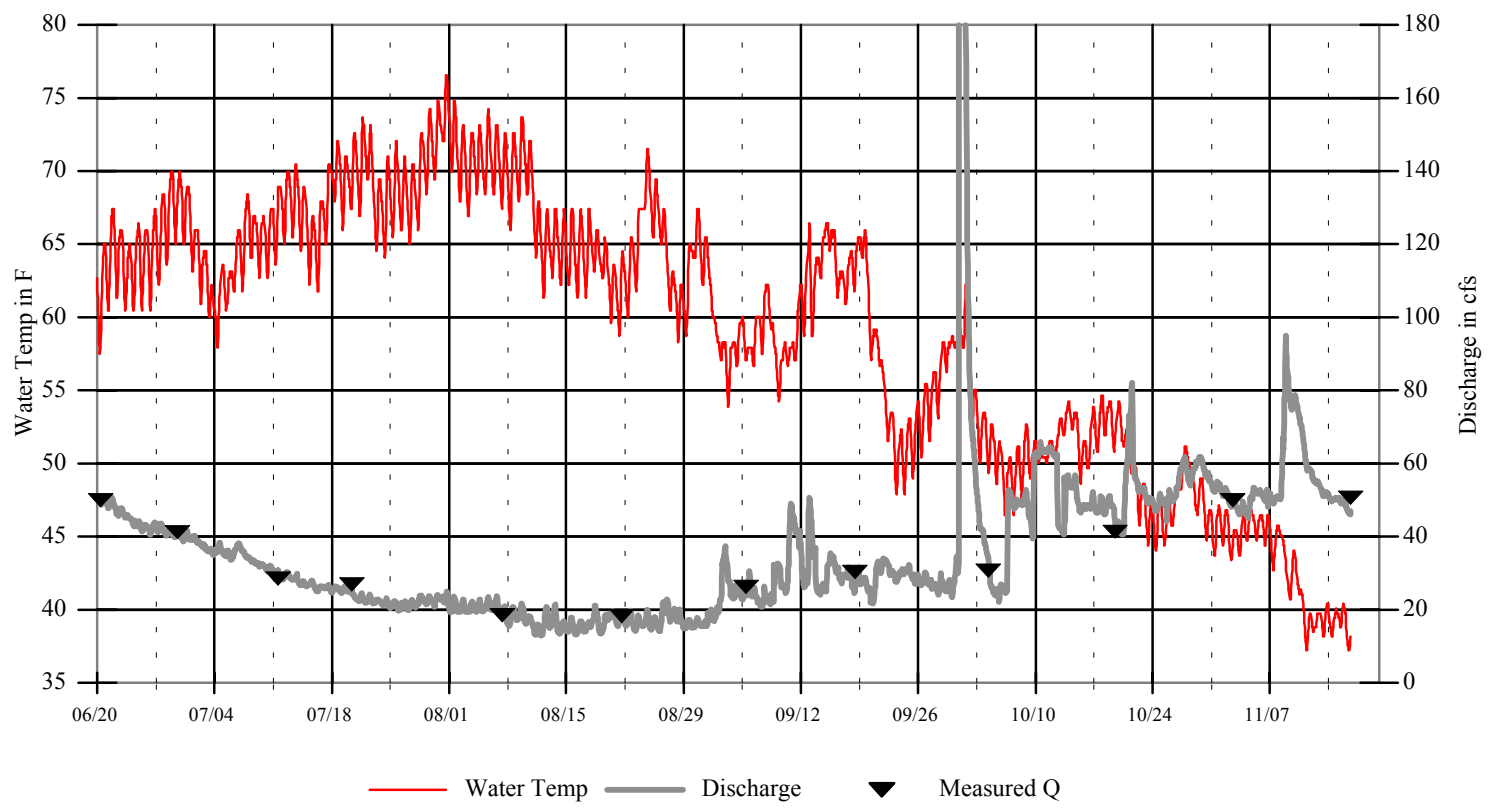

Figure 7. Yellowhawk Creek stream discharge (CFS) and daily maximum water temperatures (EF) every four hours, near confluence with the Walla Walla River (YC-8), 2000. (Field Q = manual stream discharge measurement) 


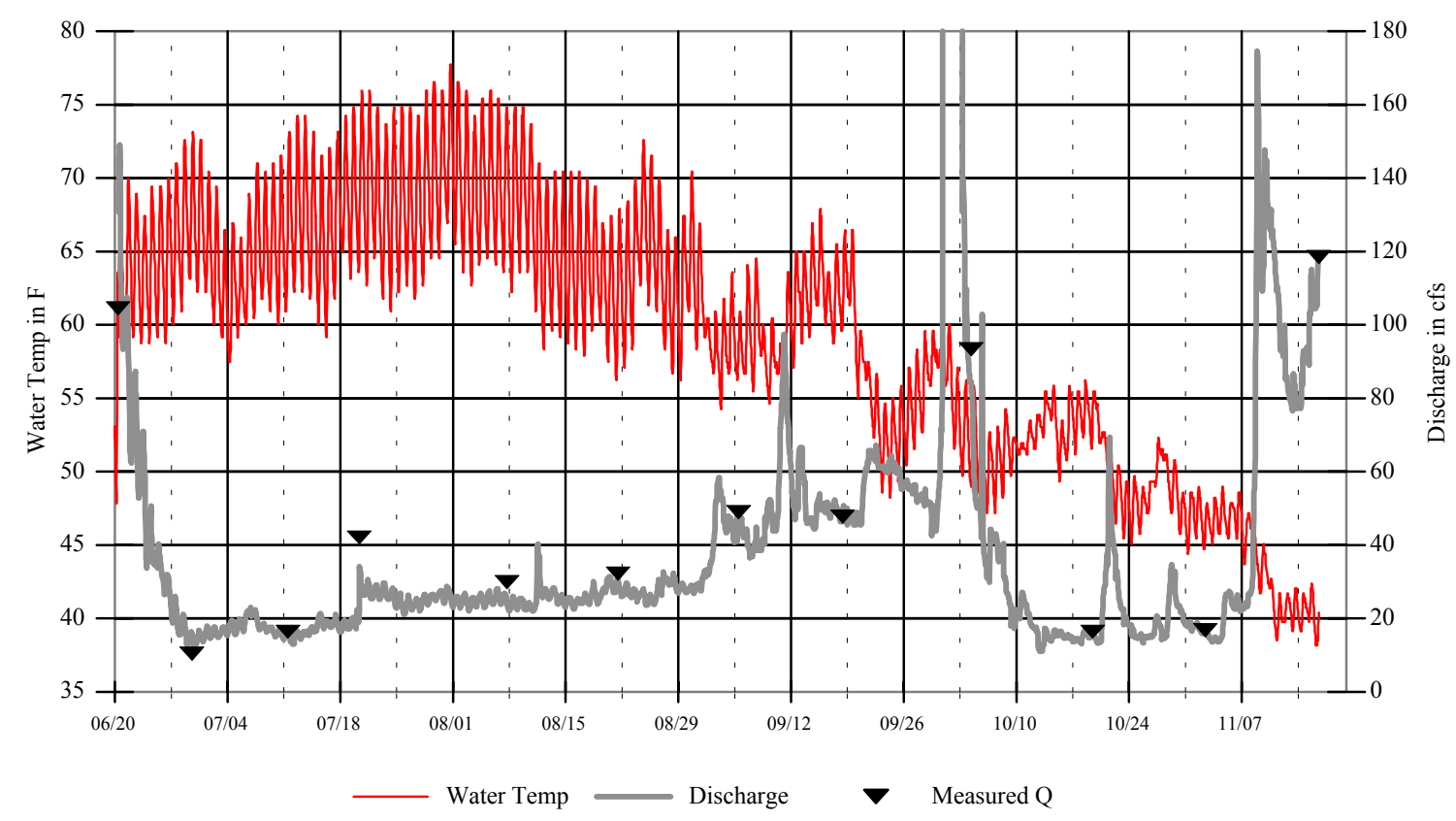

Figure 8. Walla Walla River stream discharge (CFS) and daily maximum water temperatures (EF) every four hours, below Mojonnier Bridge and Burlingame Dam (WW-6), 2000 (Field Q = manual stream discharge measurement).

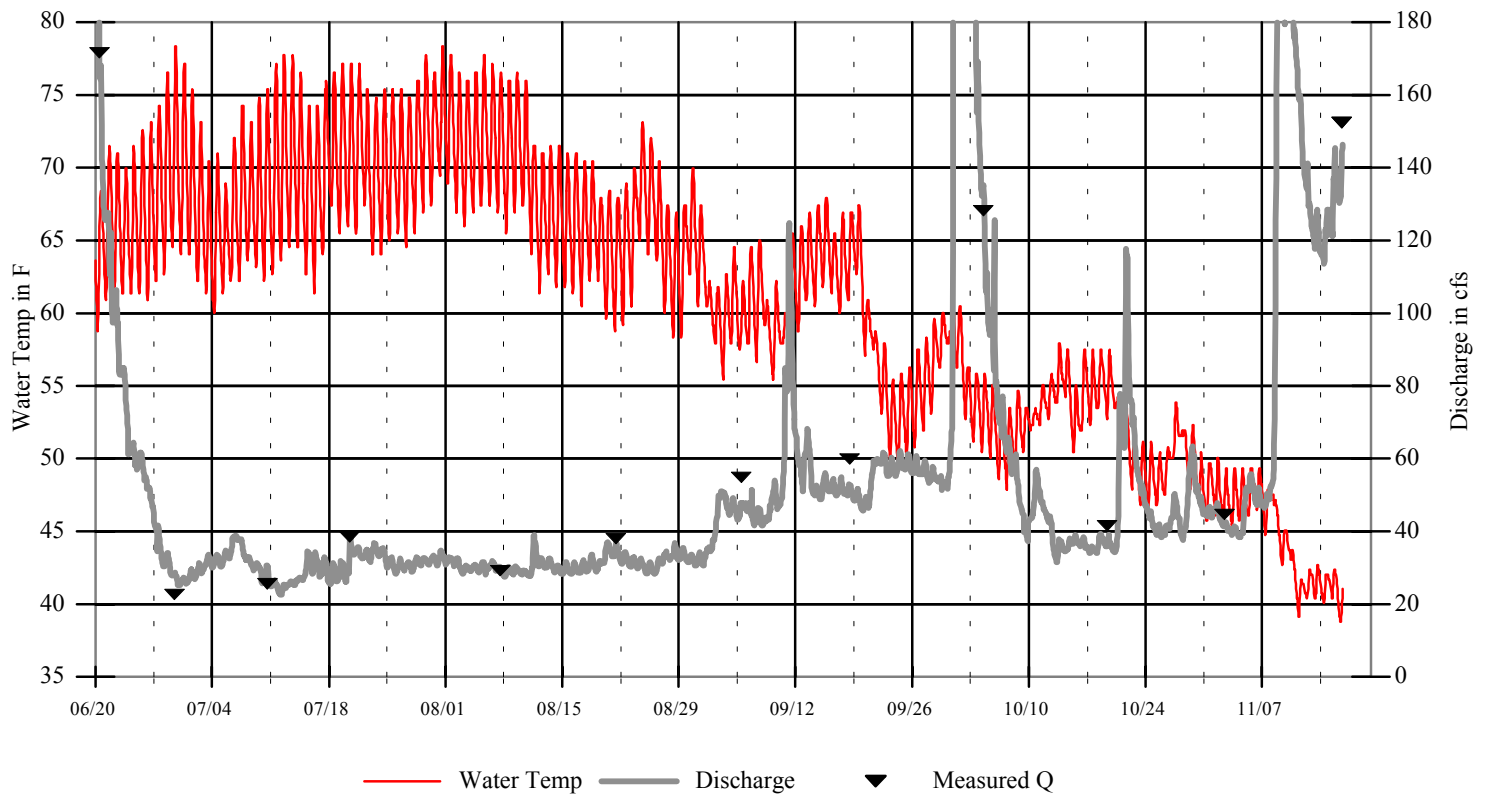

Figure 9. Walla Walla River stream discharge (CFS) and daily maximum water temperatures (EF) every four hours, above Detour Rd Bridge (ww-9), 2000 (Field Q = manual stream discharge measurement). 


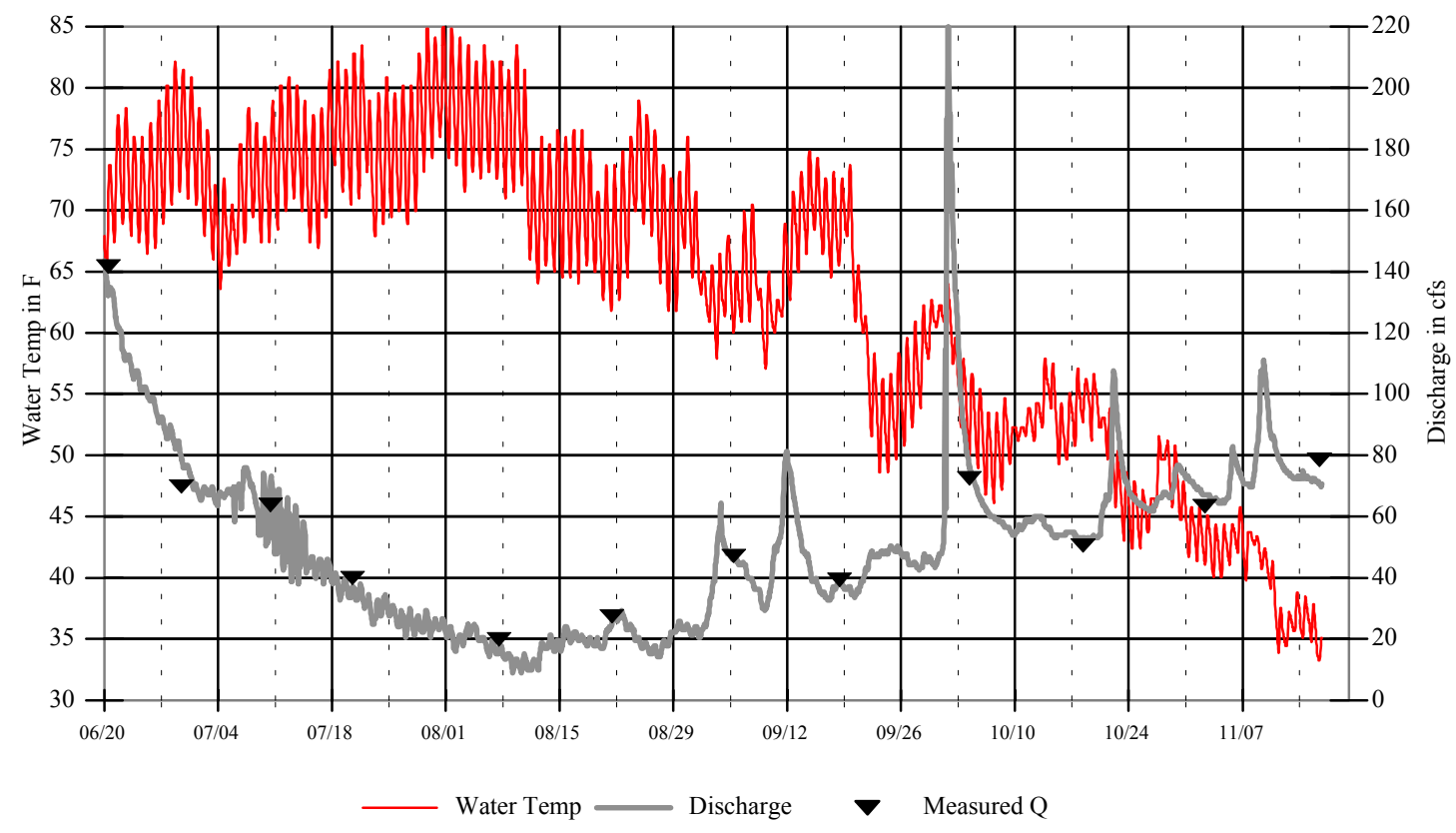

Figure 10. Touchet River stream discharge (CFS) and daily maximum water temperatures (EF) every four hours, below Sims Rd Bridge(TR-9), 2000 (Field Q = manual stream discharge measurement).

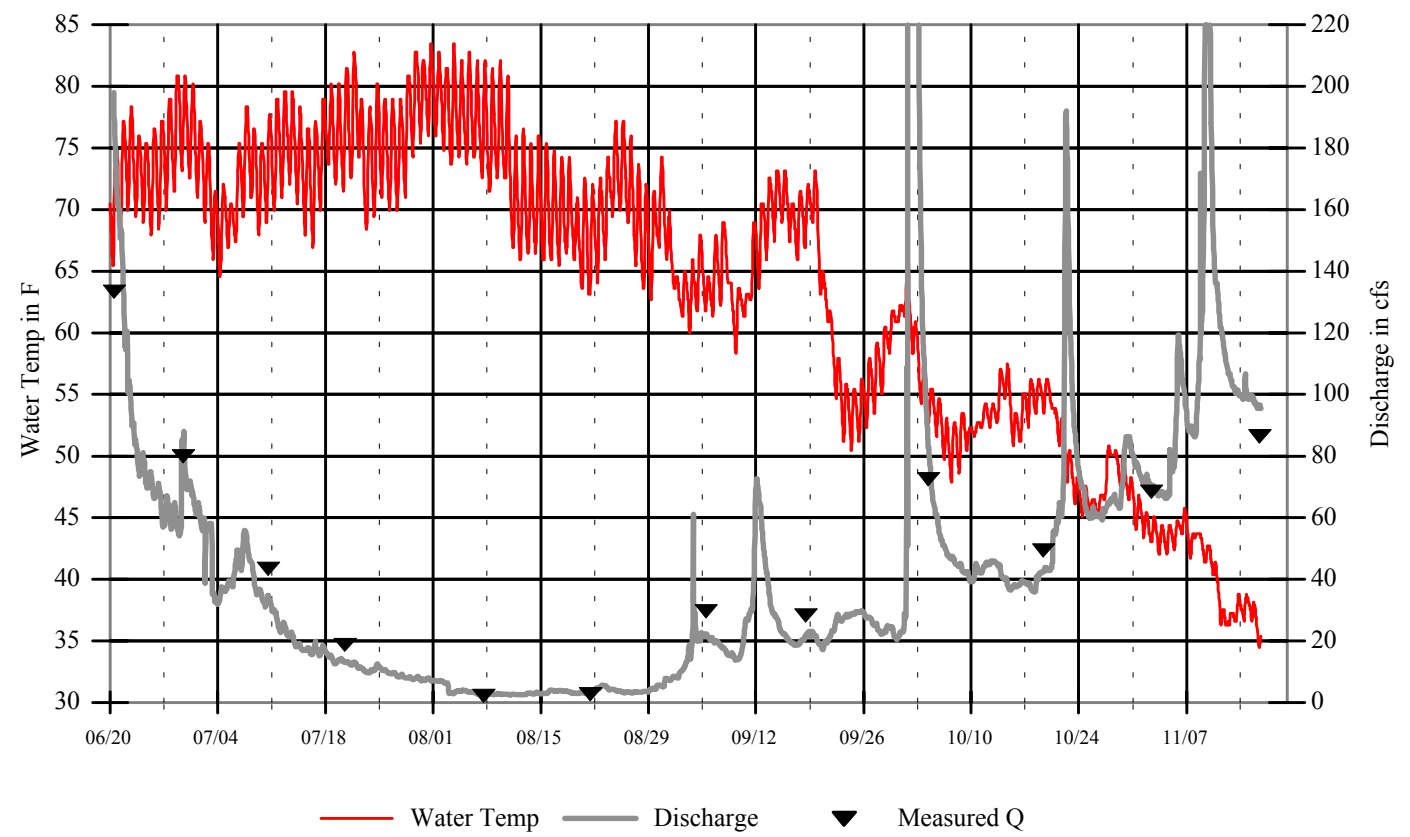

Figure 11. Touchet River stream discharge (CFS) and daily maximum water temperatures (EF) every four hours, above Cummins Rd Bridge (TR-10), 2000 (Field Q = manual stream discharge measurement). 
Preliminary results of the IFIM stream discharge modeling for the Walla Walla River and lower Mill Creek in 1999 are provided in Appendix G.

\section{Stream Temperatures}

Stream temperature monitoring for the 2000 season was focused primarily in the mainstem Walla Walla River and the Touchet River and its tributaries (Appendix C \& C1). Despite low water flows and very little precipitation, water temperatures in 2000 were similar to water temperatures in 1999 throughout the Walla Walla basin. A cold weather pattern and heavy rains during early and mid June caused water temperatures to drop throughout the basin. The low water temperatures in June interrupted the normal summer temperature development and with a declining photo-period, stream temperatures remained relatively moderate throughout the rest of the summer. Sites where maximum water temperatures were less than or equal to $65 \mathrm{EF}$ during summer months were generally located in tributaries associated with the Blue mountains; Bluewood Culvert (NFT1), Spangler Ck (SC6), NF Touchet (NFT7), Lewis Ck (LC12), Wolf Fork (WF5,WF9), Whitney Ck (WH2), Coates Creek (C1), and Upper Robinson Fork (RF4). Maximum daily temperatures at some instream monitoring sites routinely exceeded lethal temperatures for salmonids (75-84EF, Bjornn and Reiser 1991) during mid-summer, when the photo-period is long and evening cooling is brief. Sites with maximum water temperatures greater than $75 \mathrm{EF}$ included the Washington state portion of the Walla Walla River (WW2, WW6,WW8, WW9, WW10, WW11), Walla Walla River at Byrely Bridge (COE), Walla Walla River at Nine Mile (COE), Yellowhawk Creek(YC1, YC8), Birch Creek (COE), Caldwell Creek (COE), Garrison Creek $1 \& 2$ (COE), Mill Creek $1 \& 2$ (COE), Mud Creek (COE), Pine Creek (COE), the Touchet River below Dayton (TR6, TR7, TR8, TR10), Dry Creek (DC4, DC5, DC6), Lower Robinson Fork (RF14), Lower South Fork Touchet (SFT9), South Patit Creek (SFP2), and the mainstem of Coppei Creek (MC3). Sites in the mid and lower Touchet and Walla Walla rivers frequently had daily maximum temperatures that were high enough (above 68EF) to inhibit migration of adults and young, and to sharply reduce survival of embryos and fry (Bjornn and Reiser 1991,Figure 6). However, at night, temperatures would usually decrease to within reasonable physiological limits for steelhead/rainbow trout $(<65-70 \mathrm{EF})$.

\section{Water Quality}

Miscellaneous water quality measurements were taken by WDFW during the 2000 season (Appendix D). We anticipated having more water quality data, but we had equipment difficulties throughout the season.

\section{Limiting Factor Identification}

A number of barriers or impediments to salmonid passage and rearing were identified during the 2000 field season. A portion of those barriers were physical (e.g., structures or dewatered streambeds) that physically blocked salmonid movement. Physiological barriers and impediments to salmonid passage and rearing were extensive in terms of stream miles affected. The primary physiological factor was 
temperature, although high $\mathrm{pH}$ and low dissolved oxygen levels were also documented in some midor lower mainstem river reaches. Temperature possibly represents the most critical physiological barrier to salmonids, particularly for passage or rearing. Temperature related barriers for salmonids generally occur in lower areas of the Touchet and Walla Walla Rivers and their tributaries. Stream reaches with mean water temperatures exceeding $75 \mathrm{EF}$ during the summer are associated with low densities of salmonids (Mendel et. al.,1999). Most of the salmonids in these marginal thermal areas are age $0+$ rainbow/steelhead trout.

One barrier found on Russell Creek was a Civilian Conservation Corp diversion structure that was built in the 1930's or 40's. The stream bed had eroded and incised the stream channel over the years forming an impassible barrier about 15-20 ft tall. During qualitative electrofishing efforts one $190 \mathrm{~mm}$ rainbow/steelhead trout was found just below the barrier, and at least three different age classes of rainbow/steelhead trout were found downstream of the barrier. No fish of any kind were found at several sites above the barrier.

Turbidity, sedimentation, lack of pools and cover, and other habitat factors, may also present challenges to migrating, breeding and rearing salmonids. Extensive and intensive surveys of habitat conditions to identify limiting factors were deferred because of lack of adequate staff time .

\section{Fish Stock Assessment}

\section{Distribution and Abundance}

Densities and biomass of four salmonid species were calculated from electrofishing and snorkel surveys (Tables 2-6). Adult rainbow densities represent wild or unknown origin trout unless noted. Identified salmonid species included: mountain whitefish (Prosopium williamsoni), brown trout (Salmo trutta), bull trout (Salvelinus confluentus), and rainbow/steelhead trout (Oncorhynchus mykiss).

Rainbow/steelhead trout represent the most common salmonid found in the Walla Walla Basin. Age $0+$ rainbow/steelhead densities are typically higher than older age classes for most sites. Age 1+ rainbow/steelhead trout predominated in Lewis Creek, Spangler Creek, Burnt Fork, Coppei Creek, North Fork Coppei Creek, South Fork Coppei Creek, Dry Creek, Yellowhawk Creek, and Caldwell Creek. Large or "legal sized"( $\square 8$ in.) rainbow trout were found in very low densities throughout the basin. The numbers of age $0+$ steelhead found in the mainstem Walla Walla River suggests that spawning is commonly occurring within the Washington portion of the river.

Other salmonid species had a limited distribution (Tables 2-6, Appendix E). Bull trout distribution was greatest in the North Fork and the Wolf Fork of the Touchet River. In 2000, a new population of bull trout was identified in the Burnt Fork Touchet, also a few fish from two age classes were found in the South Fork Touchet. Age 1+ age class/ sub adult bull trout were observed in the South Fork around the mouth of Griffin Fork. Bull trout in the Burnt Fork ranged between 102mm-273mm, potentially representing 4 age classes (Appendix E). Low densities of bull trout were observed in 
North Fork Touchet tributaries; Lewis Creek, Spangler Creek, and Corral Creek. Mountain whitefish were rare, and they were found in low densities at only a few sites in the Walla Walla River (WW4, WW6), North Fork Touchet (NFT12, 14,15, 16), Wolf Fork (WF12) and the Touchet River (TR4 ). Brown trout were found in low densities (but included some very large individuals) in the mainstem in the North Fork Touchet, Wolf Fork, and the Touchet River around Dayton. Juvenile brown trout production appeared to be limited in 2000, but greater than observed in 1999. No juvenile chinook salmon were observed during the 2000 field season, although 4 adult Spring Chinook were observed in the Touchet River at the Dayton intake trap in late spring. One of the adult chinook had a radio transmitter and was tracked for a short time by the WDFW Snake River Lab. 
Table 2. Densities of salmonids from electrofishing sites in the Touchet River and some of its tributaries, summer and fall 2000. Sites are listed in order from upstream to downstream.

\begin{tabular}{|c|c|c|c|c|c|c|c|c|c|c|c|c|}
\hline \multirow{4}{*}{\begin{tabular}{|l} 
Stream \\
Reach
\end{tabular}} & \multirow[b]{3}{*}{ Date } & \multirow{3}{*}{$\begin{array}{c}\text { Site } \\
\text { Length }\end{array}$} & \multirow{3}{*}{$\begin{array}{l}\text { Mean } \\
\text { Width }\end{array}$} & \multirow[b]{3}{*}{ Area } & \multicolumn{8}{|c|}{ Densities (\#/100 m²) } \\
\hline & & & & & \multicolumn{4}{|c|}{ Rainbow/steelhead } & \multirow[b]{3}{*}{$\begin{array}{c}\text { Other } \\
\text { Species }^{\text {b }}\end{array}$} & \multirow{2}{*}{\multicolumn{3}{|c|}{ Age/size }} \\
\hline & & & & & & Age & ize & & & & & \\
\hline & $(\mathrm{mm} / \mathrm{dd})$ & (m) & (m) & $\left(\mathrm{m}^{2}\right)$ & $\mathbf{0 +}$ & $1+$ & $\square 8$ in & Total & & $\mathbf{0 +}$ & $1+$ & $\square 8$ in \\
\hline \multicolumn{13}{|c|}{ N. Fork Touchet } \\
\hline NFT1 & $07 / 31$ & 45.0 & 2.7 & 123.0 & 0 & 0 & 0 & 0 & BT & 4.9 & 38.2 & 0 \\
\hline NFT3 & $09 / 20$ & 37.6 & 4.3 & 160.2 & 0 & 1.2 & 0 & 1.2 & BT & 5.6 & 3.7 & 0 \\
\hline NFT6 & $08 / 03$ & 30.0 & 4.1 & 123.0 & 1.6 & 16.3 & 1.6 & 19.5 & BT & 0.8 & 0 & 0 \\
\hline NFT8 & $08 / 03$ & 30.0 & 6.3 & 187.8 & 19.2 & 20.2 & 0 & 39.4 & BT & 0 & 0.5 & 0 \\
\hline NFT9 & 08/08 & 30.0 & 6.5 & 194.0 & $14.4^{\mathrm{a}}$ & $13.4^{\mathrm{a}}$ & 0 & 27.8 & BT & 0 & 0 & 0.5 \\
\hline NFT10 & $08 / 08$ & 40.0 & 6.1 & 244.0 & 26.6 & 11.1 & 0.4 & 38.1 & & & & \\
\hline NFT11 & $08 / 02$ & 30.0 & 6.1 & 184.2 & 21.7 & $5.4^{\mathrm{a}}$ & 0.5 & 27.6 & & & & \\
\hline NFT12 & 08/09 & 35.0 & 5.9 & 207.2 & 19.8 & $9.7^{\mathrm{a}}$ & 1.0 & 30.5 & $\begin{array}{l}\text { BRT } \\
\text { MTW }\end{array}$ & $\begin{array}{l}0 \\
0\end{array}$ & $\begin{array}{l}0 \\
0\end{array}$ & $\begin{array}{l}0.5 \\
0.5\end{array}$ \\
\hline NFT13 & 08/09 & 30.0 & 6.5 & 196.2 & 20.4 & 7.1 & 0.5 & 28.0 & BRT & 0.5 & 0 & 0 \\
\hline NFT14 & 08/09 & 38.8 & 8.8 & 341.4 & $7.6^{\mathrm{a}}$ & $7.3^{\mathrm{a}}$ & 0.6 & 15.5 & MTW & 0 & 0 & 1.2 \\
\hline NFT15 & $08 / 15$ & 40.0 & 9.1 & 365.3 & $16.1^{\mathrm{a}}$ & $20.0^{\mathrm{a}}$ & 0.9 & 37.0 & $\begin{array}{l}\text { BRT } \\
\text { MTW }\end{array}$ & $\begin{array}{l}0 \\
0\end{array}$ & $\begin{array}{r}1.1 \\
0\end{array}$ & 0.8 \\
\hline NFT16 & $08 / 14$ & 37.0 & 11.6 & 427.4 & $29.3^{a}$ & 11.2 & 0.7 & 41.2 & $\begin{array}{l}\text { BRT } \\
\text { MTW }\end{array}$ & $\begin{array}{r}0.2 \\
0\end{array}$ & $\begin{array}{l}0.2 \\
0.2\end{array}$ & $\begin{array}{r}1.6^{\mathrm{a}} \\
0\end{array}$ \\
\hline NFT17 & $08 / 14$ & 37.0 & 10.2 & 376.2 & 16.5 & 5.1 & 0 & 21.6 & BRT & 0.5 & 0 & $\begin{array}{r}0.5 \\
0\end{array}$ \\
\hline \multicolumn{13}{|c|}{ Spangler Creek } \\
\hline $\mathrm{SC} 1$ & $07 / 18$ & 24.2 & 2.5 & 61.0 & 1.6 & 11.5 & 0 & 13.1 & BT & 0 & 14.8 & 0 \\
\hline SC2 & $07 / 18$ & 17.3 & 2.8 & 48.4 & 4.1 & 18.6 & 0 & 22.7 & BT & 0 & 10.3 & 0 \\
\hline SC3 & $07 / 18$ & 22.3 & 3.0 & 67.3 & 0 & 10.4 & 0 & 10.4 & BT & 0 & 4.5 & 0 \\
\hline SC4 & 08/02 & 30.6 & 2.7 & 83.8 & 6.0 & 17.9 & 0 & 23.9 & BT & 0 & 6.0 & 0 \\
\hline SC5 & 08/01 & 62.0 & 3.3 & 205.1 & 0.5 & 9.8 & 0.5 & 10.8 & & & & \\
\hline SC6 & 08/01 & 37.5 & 3.1 & 114.8 & $5.2^{\mathrm{a}}$ & 14.8 & 0 & 20.0 & & & & \\
\hline \multicolumn{13}{|c|}{ Lewis Creek } \\
\hline LC1 & $07 / 17$ & 48.0 & 2.8 & 133.6 & 0.7 & 0.7 & 0.7 & 2.1 & & & & \\
\hline LC2 & $07 / 17$ & 25.0 & 2.5 & 62.5 & 0 & 0 & 0 & 0 & & & & \\
\hline LC3 & $07 / 17$ & 30.0 & 2.6 & 80.0 & 0 & 0 & 0 & 0 & & & & \\
\hline LC4 & $07 / 17$ & 30.0 & 2.0 & 59.4 & 0 & 15.2 & 1.7 & 16.9 & & & & \\
\hline LC5 & $07 / 17$ & 30.0 & 1.9 & 56.0 & 1.8 & 12.5 & 0 & 14.3 & & & & \\
\hline LC6 & $07 / 17$ & 30.0 & 2.8 & 82.8 & 3.6 & 19.3 & 0 & 22.9 & BT & 0 & 1.2 & 0 \\
\hline LC7 & $07 / 13$ & 30.0 & 1.9 & 57.8 & 0 & 13.9 & 0 & 13.9 & BT & 0 & 3.5 & 0 \\
\hline LC8 & $07 / 13$ & 30.0 & 3.3 & 100.2 & 0 & 10.0 & 0 & 10.0 & BT & 0 & 5.0 & 0 \\
\hline LC9 & $07 / 13$ & 30.0 & 2.6 & 76.8 & 0 & 15.6 & 0 & 15.6 & BT & 0 & 1.3 & 0 \\
\hline LC10 & $07 / 12$ & 30.0 & 2.8 & 83.0 & 0 & 9.6 & 1.2 & 10.8 & & & & \\
\hline LC11 & $07 / 10$ & 30.0 & 3.1 & 94.3 & 0 & 10.6 & 1.1 & 11.7 & & & & \\
\hline LC12 & $07 / 12$ & 46.6 & 3.6 & 168.5 & 0 & 19.0 & 0 & 19.0 & & & & \\
\hline
\end{tabular}

Assessment of Salmonids and Their Habitat Conditions in

The Walla Walla River Basin of Washington: 2000 Annual Report 
Table 2. (Continued) Densities of salmonids from electrofishing sites in the Touchet River and some of its tributaries, summer and fall 2000. Sites are listed in order from upstream to downstream.

\begin{tabular}{|c|c|c|c|c|c|c|c|c|c|c|c|c|}
\hline \multirow{4}{*}{$\begin{array}{l}\text { Stream } \\
\text { Reach } \\
\text { Site } \\
\text { Name }\end{array}$} & \multirow{4}{*}{$\begin{array}{c}\text { Date } \\
(\mathrm{mm} / \mathrm{dd})\end{array}$} & \multirow{4}{*}{$\begin{array}{c}\begin{array}{c}\text { Site } \\
\text { Length }\end{array} \\
(\mathrm{m})\end{array}$} & \multirow{4}{*}{$\begin{array}{r}\text { Mean } \\
\text { Width } \\
(m)\end{array}$} & \multirow{4}{*}{$\begin{array}{l}\text { Area } \\
\left(\mathbf{m}^{2}\right)\end{array}$} & \multicolumn{8}{|c|}{ Densities (\#/100 m²) } \\
\hline & & & & & \multicolumn{4}{|c|}{ Rainbow/steelhead } & \multirow[b]{3}{*}{$\begin{array}{c}\text { Other } \\
\text { Species }^{\text {b }}\end{array}$} & \multirow{2}{*}{\multicolumn{3}{|c|}{ Age/size }} \\
\hline & & & & & \multicolumn{4}{|c|}{ Age/size } & & & & \\
\hline & & & & & $\mathbf{0 +}$ & $1+$ & $\square 8$ in & Total & & 0+ & $1+$ & $\square 8$ in \\
\hline \multicolumn{13}{|c|}{ Wolf Fork of the Touchet } \\
\hline WF2 & $08 / 10$ & 71.0 & 3.4 & 241.4 & 0 & 1.7 & 0 & 1.7 & BT & 0 & 11.2 & 0 \\
\hline WF3 & $08 / 02$ & 50.0 & 3.5 & 175.0 & 0.6 & 4.0 & 1.1 & 5.7 & BT & 8.6 & 6.3 & 0.6 \\
\hline WF4 & $08 / 02$ & 50.0 & 3.0 & 149.0 & 0 & 15.4 & 0 & 15.4 & BT & 4.0 & 8.1 & 0 \\
\hline WF5 & $08 / 02$ & 50.0 & 5.4 & 269.0 & 0.7 & 8.9 & 1.1 & 10.7 & BT & 7.1 & 13 & 0.7 \\
\hline WF6 & $08 / 02$ & 50.0 & 7.7 & 384.0 & 6.8 & 9.9 & 0 & 16.7 & BT & 0.3 & 0 & 0.3 \\
\hline WF7 & $08 / 03$ & 50.0 & 7.0 & 348.0 & 17.0 & 8.9 & 0.3 & 26.2 & & & & \\
\hline WF8 & $08 / 01$ & 40.0 & 9.2 & 366.4 & 17.7 & 9.6 & 0 & 27.3 & BT & 0 & 0 & 0.3 \\
\hline WF10 & $08 / 01$ & 50.0 & 7.5 & 374.0 & 17.9 & 8.0 & 0 & 25.9 & BRT & 1.4 & 0 & 0 \\
\hline \multirow[t]{2}{*}{ WF11 } & $07 / 25$ & 50.0 & 10.5 & 532.0 & 22.0 & 4.3 & 0.2 & 26.5 & BRT & 0.5 & 0 & 0 \\
\hline & & & & & & & & & $\mathrm{BT}$ & 0.5 & 0.3 & 0 \\
\hline \multirow[t]{3}{*}{ WF12 } & $07 / 26$ & 50.0 & 6.5 & 451.8 & 19.9 & 7.5 & 0 & 27.4 & MTW & 0 & 0 & 1.2 \\
\hline & & & & & & & & & BRT & 4.0 & 0 & 0.2 \\
\hline & & & & & & & & & BT & 0 & 0.2 & 0 \\
\hline \multicolumn{13}{|c|}{ Robinson Fork of the Touchet } \\
\hline RF3 & $07 / 19$ & 30.0 & 3.2 & 95.4 & 6.3 & 22.0 & 0 & 28.3 & & & & \\
\hline RF8 & $07 / 19$ & 30.0 & 3.7 & 111.0 & 55.0 & 13.5 & 0 & 68.5 & & & & \\
\hline RF11 & 08/02 & 30.0 & 5.6 & 166.8 & 30.0 & $7.8^{\mathrm{a}}$ & 0 & 37.8 & & & & \\
\hline RF13 & $08 / 02$ & 30.0 & 4.6 & 138.6 & 10.8 & 14.4 & 0 & 25.2 & & & & \\
\hline \multicolumn{13}{|c|}{ Burnt Fork of the S. Touchet } \\
\hline $\mathrm{BF} 3$ & 08/07 & 25.0 & 2.7 & 67.9 & 4.4 & 22.1 & 0 & 26.5 & & & & \\
\hline \multicolumn{13}{|c|}{ South Fork of the Touchet } \\
\hline SF1 & $08 / 10$ & 50.0 & 5.6 & 278 & 11.9 & 41.0 & 1.4 & 54.3 & BT & 0 & 2.2 & 0.7 \\
\hline SF2 & $08 / 10$ & 50.0 & 5.5 & 277 & 12.6 & 14.4 & 0 & 27.0 & & & & \\
\hline SF3 & $07 / 27$ & 50.0 & 4.9 & 245 & 2.0 & 9.0 & 0.8 & 11.8 & & & & \\
\hline SF4 & $08 / 24$ & 30.0 & 4.4 & 132.0 & 47.0 & 19.7 & 0.7 & 67.4 & & & & \\
\hline SF5 & $07 / 20$ & 30.0 & 6.5 & 196.2 & 14.3 & 1.0 & 0 & 15.3 & & & & \\
\hline SF6 & $07 / 27$ & 50.0 & 5.9 & 297.0 & 17.9 & 10.4 & 0 & 28.3 & & & & \\
\hline SF7 & $07 / 19$ & 35.0 & 4.7 & 165.2 & 10.3 & 14.5 & 0.6 & 25.4 & & & & \\
\hline SF8 & $07 / 20$ & 30.0 & 5.0 & 151.2 & 4.0 & 0 & 0 & 4.0 & & & & \\
\hline
\end{tabular}


Table 2. (Continued) Densities of salmonids from electrofishing sites in the Touchet River and some of its tributaries, summer and fall 2000. Sites are listed in order from upstream to downstream.

\begin{tabular}{|c|c|c|c|c|c|c|c|c|c|c|c|c|}
\hline \multirow{4}{*}{$\begin{array}{l}\text { Stream } \\
\text { Reach } \\
\text { Site } \\
\text { Name }\end{array}$} & \multirow[b]{3}{*}{ Date } & \multirow{3}{*}{$\begin{array}{c}\text { Site } \\
\text { Length }\end{array}$} & \multirow{3}{*}{$\begin{array}{l}\text { Mean } \\
\text { Width }\end{array}$} & \multirow[b]{3}{*}{ Area } & \multicolumn{8}{|c|}{ Densities (\#/100 m²) } \\
\hline & & & & & \multicolumn{4}{|c|}{ Rainbow/steelhead } & \multirow[b]{3}{*}{$\begin{array}{c}\text { Other } \\
\text { Species }^{b}\end{array}$} & \multirow{2}{*}{\multicolumn{3}{|c|}{ Age/size }} \\
\hline & & & & & & Ag & size & & & & & \\
\hline & $(\mathrm{mm} / \mathrm{dd})$ & (m) & (m) & $\left(\mathbf{m}^{2}\right)$ & $\mathbf{0 +}$ & $1+$ & $\square 8$ in & Total & & $\mathbf{0 +}$ & $1+$ & $\square 8$ in \\
\hline \multicolumn{13}{|c|}{ Touchet River } \\
\hline TR1 & $08 / 30$ & 45.0 & 11.4 & 513.0 & 2.3 & 8.2 & 3.1 & 13.6 & \multirow{4}{*}{$\begin{array}{l}\text { BRT } \\
\text { BRT } \\
\text { MTW }\end{array}$} & \multirow{4}{*}{$\begin{array}{l}0.2 \\
0.3 \\
0.2\end{array}$} & 0 & \multirow{4}{*}{$\begin{array}{r}0 \\
0.2 \\
0\end{array}$} \\
\hline TR4 & $08 / 31$ & 50.0 & 12.5 & 625.0 & 31.7 & 6.1 & 2.2 & 40.0 & & & 0 & \\
\hline & & & & & & & & & & & 0 & \\
\hline TR5 & $08 / 31$ & 40.0 & 14.4 & 576.8 & 5.4 & 1.4 & 1.0 & 7.8 & & & & \\
\hline \multicolumn{13}{|c|}{ North Coppei Creek } \\
\hline NFC1 & $08 / 17$ & 30.0 & 3.1 & 93.0 & 0 & 3.2 & 1.1 & 4.3 & & & & \\
\hline NFC3 & $08 / 17$ & 30.0 & 1.6 & 48.6 & 24.7 & 41.2 & 0 & 65.9 & & & & \\
\hline NFC5 & $08 / 17$ & 30.0 & 2.3 & 69.6 & 5.7 & 54.6 & 2.9 & 63.2 & & & & \\
\hline \multicolumn{13}{|c|}{ South Coppei Creek } \\
\hline SFC1 & $08 / 17$ & 30.0 & 4.0 & 118.8 & $9.3^{\mathrm{a}}$ & 21.9 & 0 & 31.2 & & & & \\
\hline SFC5 & $08 / 17$ & 30.0 & 3.1 & 93.6 & 4.3 & 32.1 & 0 & 36.4 & & & & \\
\hline \multicolumn{13}{|c|}{ Coppei Creek } \\
\hline $\mathrm{MC} 2$ & $08 / 17$ & 30 & 3.0 & 90.0 & 1.1 & 5.6 & 0 & 6.7 & & & & \\
\hline MC3 & $08 / 17$ & 50 & 3.0 & 148.0 & 0.7 & 4.1 & 0 & 4.8 & & & & \\
\hline
\end{tabular}


Table 3. Densities of salmonids from electrofishing sites in the Walla Walla River and some of its tributaries, summer and fall 2000. Sites are listed in order from upstream to downstream.

\begin{tabular}{|c|c|c|c|c|c|c|c|c|c|c|c|c|}
\hline \multirow{4}{*}{$\begin{array}{l}\text { Stream } \\
\text { Reach } \\
\text { Site } \\
\text { Name }\end{array}$} & \multirow[b]{3}{*}{ Date } & \multirow{3}{*}{$\begin{array}{c}\text { Site } \\
\text { Length }\end{array}$} & \multirow{3}{*}{$\begin{array}{r}\text { Mean } \\
\text { Width }\end{array}$} & \multirow[b]{3}{*}{ Area } & \multicolumn{8}{|c|}{ Densities $\left(\# / 100 \mathrm{~m}^{2}\right)$} \\
\hline & & & & & \multicolumn{4}{|c|}{ Rainbow/steelhead } & \multirow[b]{3}{*}{$\begin{array}{c}\text { Other } \\
\text { Species }^{\text {b }}\end{array}$} & \multirow{2}{*}{\multicolumn{3}{|c|}{ Age/size }} \\
\hline & & & & & \multicolumn{4}{|c|}{ Age/size } & & & & \\
\hline & $(\mathrm{mm} / \mathrm{dd})$ & (m) & (m) & $\left(\mathbf{m}^{2}\right)$ & $\mathbf{0 +}$ & $1+$ & $\square 8$ in & Total & & $\mathbf{0}+$ & $1+$ & $\square 8$ in \\
\hline \multicolumn{13}{|c|}{ Walla Walla River } \\
\hline WW1 & 08/01 & 30.6 & 5.0 & 152.4 & 12.5 & 4.6 & 0 & 17.1 & & & & \\
\hline WW3 & 08/01 & 30.0 & 8.4 & 252.6 & 1.6 & 0.8 & 0 & 2.4 & & & & \\
\hline WW4 & 08/01 & 31.0 & 5.7 & 177.3 & 3.4 & 0 & 1.1 & 4.5 & MTW & 0 & 0.6 & 0 \\
\hline WW6 & $08 / 10$ & 30.0 & 10.8 & 323.4 & 13.9 & 0.6 & 0 & 14.5 & MTW & 0.3 & 2.5 & 0 \\
\hline WW8 & $08 / 10$ & 30.0 & 5.5 & 166.2 & 2.4 & 0.6 & 0 & 3.0 & & & & \\
\hline WW9 & $08 / 10$ & 30.0 & 9.8 & 294.0 & 0 & 0 & 0 & 0 & & & & \\
\hline \multicolumn{13}{|c|}{ North Dry Creek } \\
\hline NFD2 & $08 / 24$ & 34.0 & 2.4 & 82.3 & 40.1 & 7.3 & 0 & 47.4 & & & & \\
\hline NFD3 & $08 / 29$ & 30.6 & 1.4 & 41.0 & 31.7 & 39.0 & 0 & 70.7 & & & & \\
\hline NFD4 & $08 / 29$ & 30.0 & 1.9 & 58.3 & 0 & 3.4 & 0 & 3.4 & & & & \\
\hline NFD6 & $08 / 29$ & 30.0 & 1.8 & 55.0 & 9.1 & 16.4 & 0 & 25.5 & & & & \\
\hline \multicolumn{13}{|c|}{ Dry Creek } \\
\hline DC1 & $08 / 29$ & 30.0 & 3.8 & 113.4 & 0 & 20.3 & 0 & 20.3 & & & & \\
\hline DC2 & $08 / 29$ & 30.0 & 3.7 & 111.6 & 2.7 & 5.4 & 0 & 8.1 & & & & \\
\hline DC3 & $08 / 30$ & 30.0 & 3.0 & 88.8 & 3.4 & 19.1 & 1.1 & 23.6 & & & & \\
\hline DC4 & $08 / 30$ & 40.0 & 4.7 & 189.6 & 4.2 & 11.6 & 0 & 15.8 & & & & \\
\hline \multicolumn{13}{|c|}{ Yellowhawk Creek } \\
\hline $\mathrm{YC} 1$ & $07 / 18$ & 35.0 & 6.2 & 217.7 & 0 & 0.5 & 0.5 & 1.0 & & & & \\
\hline $\mathrm{YC} 2$ & $08 / 28$ & 33.0 & 4.2 & 137.3 & 4.4 & 5.1 & 0 & 9.5 & & & & \\
\hline YC3 & $08 / 23$ & 105.0 & 4.2 & 437.5 & 2.7 & 8.7 & 0.5 & 11.9 & & & & \\
\hline YC5 & $08 / 28$ & 30.0 & 5.0 & 150.0 & 0 & 0 & 0 & 0 & & & & \\
\hline YC6 & $08 / 16$ & 30.0 & 4.7 & 140.0 & 0 & 0.7 & 0 & 0.7 & & & & \\
\hline YC7 & $08 / 28$ & 30.0 & 3.9 & 116.4 & 0.9 & 0.9 & 0 & 1.8 & & & & \\
\hline \multicolumn{13}{|c|}{ Cottonwood Creek } \\
\hline CWC1 & $07 / 10$ & 31.0 & 7.1 & 218.6 & 12.8 & 4.6 & 0 & 17.4 & & & & \\
\hline CWC2 & $07 / 12$ & 33.0 & 2.8 & 93.7 & 60.8 & 3.2 & 0 & 64.0 & & & & \\
\hline CWC3 & $07 / 06$ & 30.0 & 4.3 & 127.8 & 36.0 & 4.7 & 0 & 40.7 & & & & \\
\hline CWC6 & $07 / 05$ & 33.0 & 3.1 & 99.3 & 67.6 & 0 & 0 & 67.6 & & & & \\
\hline \multicolumn{13}{|c|}{ Caldwell Creek } \\
\hline CCC2 & $07 / 11$ & 30.0 & 1.6 & 48.0 & 0 & 2.1 & 0 & 2.1 & & & & \\
\hline
\end{tabular}


Table 3. (Continued) Densities of salmonids from electrofishing sites in the Walla Walla River and some of its tributaries, summer and fall 2000. Sites are listed in order from upstream to downstream.

\begin{tabular}{|c|c|c|c|c|c|c|c|c|c|c|c|c|}
\hline \multirow{4}{*}{$\begin{array}{l}\text { Stream } \\
\text { Reach }\end{array}$} & \multirow[b]{3}{*}{ Date } & \multirow{3}{*}{$\begin{array}{c}\text { Site } \\
\text { Length }\end{array}$} & \multirow{3}{*}{$\begin{array}{l}\text { Mean } \\
\text { Width }\end{array}$} & \multirow[b]{3}{*}{ Area } & \multicolumn{8}{|c|}{ Densities (\#/100 m²) } \\
\hline & & & & & \multicolumn{4}{|c|}{ Rainbow/steelhead } & \multirow[b]{3}{*}{$\begin{array}{c}\text { Other } \\
\text { Species }^{\text {b }}\end{array}$} & \multirow{2}{*}{\multicolumn{3}{|c|}{ Age/size }} \\
\hline & & & & & & & size & & & & & \\
\hline & $(\mathbf{m m} / \mathbf{d d})$ & (m) & (m) & $\left(\mathbf{m}^{2}\right)$ & $\mathbf{0 +}$ & $1+$ & $\square 8$ in & Total & & $\mathbf{0}+$ & $1+$ & $\square 8$ in \\
\hline \multicolumn{13}{|c|}{ Mill Creek } \\
\hline MC16 & $08 / 22$ & 56.2 & 3.4 & 191.1 & 0 & 0 & 0 & 0 & & & & \\
\hline MC20 & $08 / 22$ & 30.0 & 3.3 & 99.0 & 2.0 & 0 & 0 & 0 & & & & \\
\hline MC21 & $07 / 10$ & 30.0 & 6.1 & 183.0 & 1.6 & 0 & 0 & 0 & & & & \\
\hline \multicolumn{13}{|c|}{ East Little Walla Walla } \\
\hline ELW1 & $07 / 17$ & 30.0 & 3.9 & 117.0 & 0.9 & 0 & 0 & 0.9 & & & & \\
\hline ELW2 & $07 / 17$ & 30.0 & 3.4 & 102.0 & 0 & 1.0 & 0 & 1.0 & & & & \\
\hline ELW3 & $07 / 17$ & 30.0 & 3.7 & 111.0 & 0 & 0 & 0 & 0 & & & & \\
\hline \multicolumn{13}{|c|}{ Doan Creek } \\
\hline DC2 & $08 / 15$ & 30 & 1.1 & 33.0 & 9.1 & 0 & 0 & 9.1 & & & & \\
\hline
\end{tabular}


Table 4. Biomass of salmonids from electrofishing sites in the Touchet River and some of its tributaries, summer and fall 2000. Sites are listed in order from upstream to downstream.

Biomass $\left(\mathrm{g} / 100 \mathrm{~m}^{2}\right)$

\begin{tabular}{|c|c|c|c|c|c|c|c|c|c|c|c|c|}
\hline \multirow{3}{*}{$\begin{array}{l}\text { Stream } \\
\text { Reach } \\
\text { Site } \\
\text { Name }\end{array}$} & \multirow{3}{*}{$\begin{array}{c}\text { Date } \\
(\mathrm{mm} / \mathrm{dd})\end{array}$} & \multirow{3}{*}{$\begin{array}{c}\begin{array}{c}\text { Site } \\
\text { Length }\end{array} \\
(\mathrm{m})\end{array}$} & \multirow{3}{*}{$\begin{array}{r}\text { Mean } \\
\text { Width } \\
(m)\end{array}$} & \multirow{3}{*}{$\begin{array}{l}\text { Area } \\
\left(\mathbf{m}^{2}\right)\end{array}$} & \multicolumn{4}{|c|}{ Rainbow/steelhead } & \multirow{3}{*}{$\begin{array}{l}\text { Other } \\
\text { Species }\end{array}$} & \multirow{2}{*}{\multicolumn{3}{|c|}{ Age/size }} \\
\hline & & & & & \multicolumn{4}{|c|}{ Age/size } & & & & \\
\hline & & & & & 0+ & $1+$ & $\square 8$ in & Total & & $\mathbf{0 +}$ & $1+$ & $\square 8$ in \\
\hline \multicolumn{13}{|c|}{ N. Fork Touchet } \\
\hline NFT1 & $07 / 31$ & 45.0 & 2.7 & 123.0 & 0 & 0 & 0 & 0 & BT & 3.0 & 718.4 & 0 \\
\hline NFT3 & $09 / 20$ & 37.6 & 4.3 & 160.2 & 0 & 29.2 & 0 & 29.2 & BT & 5.6 & 109.0 & 0 \\
\hline NFT6 & $08 / 03$ & 30.0 & 4.1 & 123.0 & 3.7 & 443.1 & 257.8 & 704.6 & BT & 12. & 0 & 0 \\
\hline NFT8 & $08 / 03$ & 30.0 & 6.3 & 187.8 & 39.1 & 501.4 & 0 & 540.5 & BT & 8 & 51.9 & 0 \\
\hline NFT9 & 08/08 & 30.0 & 6.5 & 194.0 & $14.2^{\mathrm{a}}$ & $338.4^{\mathrm{a}}$ & 0 & 352.6 & BT & 0 & 0 & 125.3 \\
\hline NFT10 & 08/08 & 40.0 & 6.1 & 244.0 & 72.9 & 286.0 & 66.4 & 425.3 & & 0 & & \\
\hline NFT11 & 08/02 & 30.0 & 6.1 & 184.2 & 74.7 & $125.5^{\mathrm{a}}$ & 55.1 & 255.3 & & & & \\
\hline NFT12 & 08/09 & 35.0 & 5.9 & 207.2 & 57.2 & $288.1^{\mathrm{a}}$ & 149.6 & 494.9 & $\begin{array}{l}\text { BRT } \\
\text { MTW }\end{array}$ & $\begin{array}{l}0 \\
0\end{array}$ & $\begin{array}{l}0 \\
0\end{array}$ & $\begin{array}{r}1204.1 \\
142.6\end{array}$ \\
\hline NFT13 & 08/09 & 30.0 & 6.5 & 196.2 & 58.1 & 232.8 & 63.0 & 353.9 & BRT & 3.6 & 0 & 0 \\
\hline NFT14 & 08/09 & 38.8 & 8.8 & 341.4 & $29.8^{\mathrm{a}}$ & $245.0^{\mathrm{a}}$ & 93.2 & 368.0 & MTW & 1.6 & 0 & 277.1 \\
\hline NFT15 & 08/15 & 40.0 & 9.1 & 365.3 & $76.6^{a}$ & $730.7^{\mathrm{a}}$ & 209.1 & 1016.4 & $\begin{array}{l}\text { BRT } \\
\text { MTW }\end{array}$ & $\begin{array}{l}0 \\
0\end{array}$ & $\begin{array}{r}16.0 \\
0\end{array}$ & $\begin{array}{r}1355.5 \\
496.3\end{array}$ \\
\hline NFT16 & $08 / 14$ & 37.0 & 11.6 & 427.4 & $106.1^{\mathrm{a}}$ & 421.1 & 12.8 & 540.0 & $\begin{array}{l}\text { BRT } \\
\text { MTW }\end{array}$ & $\begin{array}{r}3.9 \\
0\end{array}$ & $\begin{array}{r}2.5 \\
17.2\end{array}$ & $\begin{array}{r}0 \\
520.5\end{array}$ \\
\hline NFT17 & $08 / 14$ & 37.0 & 10.2 & 376.2 & 56.6 & 178.1 & 0 & 234.7 & BRT & 4.0 & 0 & 0 \\
\hline \multicolumn{13}{|c|}{ Spangler Creek } \\
\hline $\mathrm{SC} 1$ & $07 / 18$ & 24.2 & 2.5 & 61.0 & 3.1 & 393.5 & 0 & 396.6 & BT & 0 & 128.1 & 0 \\
\hline $\mathrm{SC} 2$ & $07 / 18$ & 17.3 & 2.8 & 48.4 & 11.1 & 671.1 & 0 & 688.2 & BT & 0 & 197.7 & 0 \\
\hline $\mathrm{SC} 3$ & 07/18 & 22.3 & 3.0 & 67.3 & 0 & 271.7 & 0 & 271.7 & BT & 0 & 91.6 & 0 \\
\hline SC4 & $08 / 02$ & 30.6 & 2.7 & 83.8 & 20.5 & 484.3 & 0 & 504.8 & BT & 0 & 57.6 & 0 \\
\hline SC5 & 08/01 & 62.0 & 3.3 & 205.1 & 2.1 & 284.2 & 45.2 & 331.5 & & & & \\
\hline SC6 & 08/01 & 37.5 & 3.1 & 114.8 & $24.6^{\mathrm{a}}$ & 420.1 & 0 & 444.7 & & & & \\
\hline \multicolumn{13}{|c|}{ Lewis Creek } \\
\hline $\mathrm{LC} 1$ & $07 / 17$ & 48.0 & 2.8 & 133.6 & 1.9 & 5.2 & 71.8 & 78.9 & & & & \\
\hline LC2 & $07 / 17$ & 25.0 & 2.5 & 62.5 & 0 & 0 & 0 & 0 & & & & \\
\hline LC3 & $07 / 17$ & 30.0 & 2.6 & 80.0 & 0 & 0 & 0 & 0 & & & & \\
\hline LC4 & $07 / 17$ & 30.0 & 2.0 & 59.4 & 0 & 508.0 & 147.1 & 655.1 & & & & \\
\hline LC5 & $07 / 17$ & 30.0 & 1.9 & 56.0 & 7.1 & 352.6 & 0 & 359.7 & & & & \\
\hline LC6 & $07 / 17$ & 30.0 & 2.8 & 82.8 & 14.6 & 661.8 & 0 & 676.4 & $\mathrm{BT}$ & 0 & 25.4 & 0 \\
\hline LC7 & $07 / 13$ & 30.0 & 1.9 & 57.8 & 0 & 349.4 & 0 & 349.4 & $\mathrm{BT}$ & 0 & 76.4 & 0 \\
\hline LC8 & $07 / 13$ & 30.0 & 3.3 & 100.2 & 0 & 347.1 & 0 & 347.1 & $\mathrm{BT}$ & 0 & 117.7 & 0 \\
\hline LC9 & $07 / 13$ & 30.0 & 2.6 & 76.8 & 0 & 550.2 & 0 & 550.2 & $\mathrm{BT}$ & 0 & 25.0 & 0 \\
\hline LC10 & $07 / 12$ & 30.0 & 2.8 & 83.0 & 0 & 198.3 & 113.4 & 311.7 & & & & \\
\hline LC11 & $07 / 10$ & 30.0 & 3.1 & 94.3 & 0 & 238.6 & 101.8 & 340.4 & & & & \\
\hline LC12 & $07 / 12$ & 46.6 & 3.6 & 168.5 & 0 & 565.3 & 0 & 565.3 & & & & \\
\hline
\end{tabular}

Calculated using the sum of the passes due to poor reduction between successive passes, minimum estimates only. $\mathrm{BT}=$ Bull Trout; BRT $=$ Brown Trout; MTW $=$ White Fish 
Table 4. (Continued) Biomass of salmonids from electrofishing sites in the Touchet River and some of its tributaries, summer and fall 2000. Sites are listed in order from upstream to downstream.

\begin{tabular}{|c|c|c|c|c|c|c|c|c|c|c|c|c|}
\hline \multirow{4}{*}{$\begin{array}{l}\text { Strea } \\
\text { m } \\
\text { Reach } \\
\text { Site } \\
\text { Name }\end{array}$} & \multirow{4}{*}{$\begin{array}{c}\text { Date } \\
(\mathrm{mm} / \mathrm{dd})\end{array}$} & \multirow{4}{*}{$\begin{array}{c}\begin{array}{c}\text { Site } \\
\text { Length }\end{array} \\
\text { (m) }\end{array}$} & \multirow{4}{*}{$\begin{array}{r}\begin{array}{r}\text { Mean } \\
\text { Width }\end{array} \\
(m)\end{array}$} & \multirow{4}{*}{$\begin{array}{l}\text { Area } \\
\left(\mathrm{m}^{2}\right)\end{array}$} & \multicolumn{8}{|c|}{$\operatorname{Biomass}\left(\mathrm{g} / 100 \mathrm{~m}^{2}\right)$} \\
\hline & & & & & \multicolumn{4}{|c|}{ Rainbow/steelhead } & \multirow{3}{*}{$\begin{array}{l}\text { Other } \\
\text { Species }\end{array}$} & \multirow{2}{*}{\multicolumn{3}{|c|}{ Age/size }} \\
\hline & & & & & \multicolumn{4}{|c|}{ Age/size } & & & & \\
\hline & & & & & 0+ & $1+$ & $\square 8$ in & Total & & $\mathbf{0 +}$ & $1+$ & $\square 8$ in \\
\hline \multicolumn{13}{|c|}{ Wolf Fork of the Touchet } \\
\hline WF2 & $08 / 10$ & 71.0 & 3.4 & 241.4 & NA & NA & NA & NA & BT & NA & NA & NA \\
\hline WF3 & 08/02 & 50.0 & 3.5 & 175.0 & 1.2 & 226.6 & 146.5 & 374.3 & BT & 7.9 & 204.2 & NA \\
\hline WF4 & 08/02 & 50.0 & 3.0 & 149.0 & 0 & 385.3 & 0 & 385.3 & BT & 3.2 & 185.7 & 0 \\
\hline WF5 & 08/02 & 50.0 & 5.4 & 269.0 & 0.3 & 144.7 & 165.0 & 310.0 & BT & 14.1 & 188.5 & NA \\
\hline WF6 & 08/02 & 50.0 & 7.7 & 384.0 & 7.8 & 233.2 & 0 & 241.0 & BT & 5.1 & 0 & 17.8 \\
\hline WF7 & $08 / 03$ & 50.0 & 7.0 & 348.0 & 27.2 & 258.0 & 45.0 & 330.2 & & & & \\
\hline WF8 & 08/01 & 40.0 & 9.2 & 366.4 & 31.5 & 167.2 & 0 & 198.7 & BT & 0 & 0 & 49.7 \\
\hline WF10 & 08/01 & 50.0 & 7.5 & 374.0 & NA & NA & NA & NA & BRT & NA & NA & NA \\
\hline \multirow[t]{2}{*}{ WF11 } & $07 / 25$ & 50.0 & 10.5 & 532.0 & 63.1 & 96.9 & 22.2 & 182.2 & BRT & 2.3 & 0 & 0 \\
\hline & & & & & & & & & BT & 2.7 & 8.5 & 0 \\
\hline \multirow[t]{3}{*}{ WF12 } & $07 / 26$ & 50.0 & 6.5 & 451.8 & NA & NA & NA & NA & MTW & NA & NA & NA \\
\hline & & & & & & & & & BRT & NA & NA & NA \\
\hline & & & & & & & & & $\mathrm{BT}$ & NA & NA & NA \\
\hline \multicolumn{13}{|c|}{ Robinson Fork of the Touchet } \\
\hline RF3 & $07 / 19$ & 30.0 & 3.2 & 95.4 & 2.1 & 556.5 & 0 & 558.6 & & & & \\
\hline RF8 & 07/19 & 30.0 & 3.7 & 111.0 & 55.7 & 186.5 & 0 & 242.2 & & & & \\
\hline RF11 & 08/02 & 30.0 & 5.6 & 166.8 & 50.5 & $184.6^{\mathrm{a}}$ & 0 & 235.1 & & & & \\
\hline RF13 & $08 / 02$ & 30.0 & 4.6 & 138.6 & 23.5 & 261.7 & 0 & 285.2 & & & & \\
\hline \multicolumn{13}{|c|}{ Burnt Fork of the S. Touchet } \\
\hline BF3 & $08 / 07$ & 25.0 & 2.7 & 67.9 & 15.0 & 713.5 & 0 & 728.5 & & & & \\
\hline \multicolumn{13}{|c|}{ South Fork of the Touchet } \\
\hline SF1 & $08 / 10$ & 50.0 & 5.6 & 278 & 23.8 & 880.4 & 143.6 & 1047.8 & & & & \\
\hline SF2 & $08 / 10$ & 50.0 & 5.5 & 277 & 23.2 & 255.5 & 0 & 278.7 & & & & \\
\hline SF3 & $07 / 27$ & 50.0 & 4.9 & 245 & 4.6 & 293.6 & 124.1 & 422.3 & & & & \\
\hline SF4 & $08 / 24$ & 30.0 & 4.4 & 132.0 & 164.5 & 490.5 & 72.6 & 727.6 & & & & \\
\hline SF5 & $07 / 20$ & 30.0 & 6.5 & 196.2 & 42.2 & 22.2 & 0 & 64.4 & & & & \\
\hline SF6 & $07 / 27$ & 50.0 & 5.9 & 297.0 & 57.2 & 203.2 & 0 & 260.4 & & & & \\
\hline SF7 & $07 / 19$ & 35.0 & 4.7 & 165.2 & 25.4 & 573.6 & 122.3 & 721.3 & & & & \\
\hline SF8 & $07 / 20$ & 30.0 & 5.0 & 151.2 & 9.2 & 0 & 0 & 9.2 & & & & \\
\hline
\end{tabular}


Table 4. (Continued) Biomass of salmonids from electrofishing sites in the Touchet River and some of its tributaries, summer and fall 2000. Sites are listed in order from upstream to downstream.

\begin{tabular}{|c|c|c|c|c|c|c|c|c|c|c|c|c|}
\hline \multirow{4}{*}{$\begin{array}{l}\begin{array}{l}\text { Stream } \\
\text { Reach }\end{array} \\
\text { Site } \\
\text { Name }\end{array}$} & \multirow{4}{*}{$\begin{array}{c}\text { Date } \\
(\mathrm{mm} / \mathrm{dd})\end{array}$} & \multirow{4}{*}{$\begin{array}{c}\begin{array}{c}\text { Site } \\
\text { Length }\end{array} \\
(\mathrm{m})\end{array}$} & \multirow{4}{*}{$\begin{array}{r}\text { Mean } \\
\text { Width } \\
(m)\end{array}$} & \multirow{4}{*}{$\begin{array}{l}\text { Area } \\
\left(\mathbf{m}^{2}\right)\end{array}$} & \multicolumn{8}{|c|}{$\operatorname{Biomass}\left(\mathrm{g} / 100 \mathrm{~m}^{2}\right)$} \\
\hline & & & & & \multicolumn{4}{|c|}{ Rainbow/steelhead } & \multirow[b]{3}{*}{$\begin{array}{c}\text { Other } \\
\text { Species }^{b}\end{array}$} & \multirow{2}{*}{\multicolumn{3}{|c|}{ Age/size }} \\
\hline & & & & & \multicolumn{4}{|c|}{ Age/size } & & & & \\
\hline & & & & & 0+ & $1+$ & $\square 8$ in & Total & & $\mathbf{0 +}$ & $1+$ & $\square 8$ in \\
\hline \multicolumn{13}{|c|}{ Touchet River } \\
\hline TR1 & $08 / 30$ & 45.0 & 11.4 & 513.0 & 27.4 & 435.0 & 503.2 & 965.6 & \multirow{3}{*}{$\begin{array}{l}\text { BRT } \\
\text { BRT } \\
\text { MTW }\end{array}$} & \multirow{3}{*}{$\begin{array}{l}4.0 \\
4.6 \\
2.6\end{array}$} & \multirow{3}{*}{$\begin{array}{l}0 \\
0 \\
0\end{array}$} & \multirow{3}{*}{$\begin{array}{r}0 \\
34.6 \\
0\end{array}$} \\
\hline TR4 & $08 / 31$ & 50.0 & 12.5 & 625.0 & 234.6 & 308.5 & 527.5 & 1070.6 & & & & \\
\hline TR5 & $08 / 31$ & 40.0 & 14.4 & 576.8 & 40.3 & 68.5 & 144.8 & 253.6 & & & & \\
\hline \multicolumn{13}{|c|}{ North Coppei Creek } \\
\hline NFC1 & $08 / 17$ & 30.0 & 3.1 & 93.0 & 0 & 85.8 & 108.4 & 194.2 & & & & \\
\hline NFC3 & 08/17 & 30.0 & 1.6 & 48.6 & 75.1 & 723.0 & 0 & 798.1 & & & & \\
\hline NFC5 & $08 / 17$ & 30.0 & 2.3 & 69.6 & 28.6 & 1036 & 562.8 & 1627.4 & & & & \\
\hline \multicolumn{13}{|c|}{ South Coppei Creek } \\
\hline SFC1 & $08 / 17$ & 30.0 & 4.0 & 118.8 & $36.3^{\mathrm{a}}$ & 412.6 & 0 & 448.9 & & & & \\
\hline SFC5 & $08 / 17$ & 30.0 & 3.1 & 93.6 & 18.3 & 655.8 & 0 & 674.1 & & & & \\
\hline \multicolumn{13}{|c|}{ Coppei Creek } \\
\hline MC2 & $08 / 17$ & 30 & 3.0 & 90.0 & 4.4 & 168.6 & 0 & 173.0 & & & & \\
\hline MC3 & $08 / 17$ & 50 & 3.0 & 148.0 & 4.1 & 160.9 & 0 & 165.0 & & & & \\
\hline
\end{tabular}


Table 5. Biomass of salmonids from electrofishing sites in the Walla Walla River and some of its tributaries, summer and fall 2000. Sites are listed in order from upstream to downstream.

\begin{tabular}{|c|c|c|c|c|c|c|c|c|c|c|c|c|}
\hline \multirow{4}{*}{$\begin{array}{l}\text { Stream } \\
\text { Reach }\end{array}$} & \multirow[b]{3}{*}{ Date } & \multirow{3}{*}{$\begin{array}{c}\text { Site } \\
\text { Length }\end{array}$} & \multirow{3}{*}{$\begin{array}{r}\text { Mean } \\
\text { Width }\end{array}$} & \multirow[b]{3}{*}{ Area } & \multicolumn{8}{|c|}{ Biomass $\left(\mathrm{g} / 100 \mathrm{~m}^{2}\right)$} \\
\hline & & & & & \multicolumn{4}{|c|}{ Rainbow/steelhead } & \multirow[b]{3}{*}{$\begin{array}{c}\text { Other } \\
\text { Species }^{\text {b }}\end{array}$} & \multirow{2}{*}{\multicolumn{3}{|c|}{ Age/size }} \\
\hline & & & & & \multicolumn{4}{|c|}{ Age/size } & & & & \\
\hline & $(\mathrm{mm} / \mathrm{dd})$ & (m) & (m) & $\left(\mathbf{m}^{2}\right)$ & $\mathbf{0 +}$ & $1+$ & $\square 8$ in & Total & & $\mathbf{0 +}$ & $1+$ & $\square 8$ in \\
\hline \multicolumn{13}{|c|}{ Walla Walla River } \\
\hline WW1 & $08 / 01$ & 30.6 & 5.0 & 152.4 & 69.1 & 164.8 & 0 & 233.9 & & & & \\
\hline WW3 & 08/01 & 30.0 & 8.4 & 252.6 & 12.2 & 32.3 & 0 & 44.5 & & & & \\
\hline WW4 & 08/01 & 31.0 & 5.7 & 177.3 & 22.1 & 0 & 159.6 & 181.7 & MTW & 0 & 7.7 & 0 \\
\hline WW6 & $08 / 10$ & 30.0 & 10.8 & 323.4 & 89.3 & 30.0 & 0 & 119.3 & MTW & 12.1 & 24.1 & 0 \\
\hline WW8 & $08 / 10$ & 30.0 & 5.5 & 166.2 & 19.7 & 52.9 & 0 & 72.6 & & & & \\
\hline WW9 & $08 / 10$ & 30.0 & 9.8 & 294.0 & & & & & & & & \\
\hline \multicolumn{13}{|c|}{ North Dry Creek } \\
\hline NFD2 & $08 / 24$ & 34.0 & 2.4 & 82.3 & 97.5 & 246.7 & 0 & 344.2 & & & & \\
\hline NFD3 & $08 / 29$ & 30.6 & 1.4 & 41.0 & 122.9 & 1180 & 0 & 1302.9 & & & & \\
\hline NFD4 & $08 / 29$ & 30.0 & 1.9 & 58.3 & 0 & 68.2 & 0 & 68.2 & & & & \\
\hline NFD6 & $08 / 29$ & 30.0 & 1.8 & 55.0 & 44.7 & 451.3 & 0 & 496.0 & & & & \\
\hline \multicolumn{13}{|c|}{ Dry Creek } \\
\hline DC1 & $08 / 29$ & 30.0 & 3.8 & 113.4 & 0 & 480.7 & 0 & 480.7 & & & & \\
\hline DC2 & 08/29 & 30.0 & 3.7 & 111.6 & 13.9 & 93.0 & 0 & 106.9 & & & & \\
\hline DC3 & $08 / 30$ & 30.0 & 3.0 & 88.8 & 17.0 & 504.0 & 161.5 & 682.5 & & & & \\
\hline DC4 & $08 / 30$ & 40.0 & 4.7 & 189.6 & 15.8 & 301.4 & 0 & 317.2 & & & & \\
\hline \multicolumn{13}{|c|}{ Yellowhawk Creek } \\
\hline $\mathrm{YC} 1$ & $07 / 18$ & 35.0 & 6.2 & 217.7 & 0 & 11.2 & 98.8 & 110.0 & & & & \\
\hline YC2 & $08 / 28$ & 33.0 & 4.2 & 137.3 & 15.1 & 163.5 & 0 & 178.6 & & & & \\
\hline YC3 & $08 / 23$ & 105.0 & 4.2 & 437.5 & 6.0 & 241.8 & 48.1 & 295.9 & & & & \\
\hline YC6 & $08 / 16$ & 30.0 & 4.7 & 140.0 & 0 & 14.4 & 0 & 14.4 & & & & \\
\hline YC7 & $08 / 28$ & 30.0 & 3.9 & 116.4 & 3.9 & 30.2 & 0 & 34.1 & & & & \\
\hline \multicolumn{13}{|c|}{ Cootonwood Creek } \\
\hline $\mathrm{CWC1}$ & $07 / 10$ & 31.0 & 7.1 & 218.6 & 56.5 & 157.5 & 0 & 214.0 & & & & \\
\hline CWC2 & 07/12 & 33.0 & 2.8 & 93.7 & 372.8 & 92.0 & 0 & 464.8 & & & & \\
\hline CWC3 & $07 / 16$ & 30.0 & 4.3 & 127.8 & 165.0 & 85.2 & 0 & 250.2 & & & & \\
\hline CWC6 & $07 / 15$ & 30.0 & 3.1 & 91.8 & 358.8 & 0 & 0 & 358.8 & & & & \\
\hline \multicolumn{13}{|c|}{ Caldwell Creek } \\
\hline $\mathrm{CCC} 2$ & 07/11 & 30.0 & 1.6 & 48.0 & 0 & 54.0 & 0 & 54.0 & & & & \\
\hline \multicolumn{13}{|c|}{ East Little Walla Walla } \\
\hline ELW1 & $07 / 17$ & 30.0 & 3.9 & 117.0 & 3.3 & 0 & 0 & 3.3 & & & & \\
\hline ELW2 & $07 / 17$ & 30.0 & 3.4 & 102.0 & 0 & 77.3 & 0 & 77.3 & & & & \\
\hline \multicolumn{13}{|c|}{ Doan Creek } \\
\hline DC2 & $08 / 15$ & 30 & 1.1 & 33.0 & 115.6 & 0 & 0 & 115.6 & & & & \\
\hline
\end{tabular}




\section{Electrofishing}

Densities of rainbow/steelhead trout ranged from 0 to 70.7 fish per $100 \mathrm{~m}^{2}$ at sampled sites (Tables 2 \& 3). Sub-yearling (age 0+) were the most abundant age class at sites in the mainstem and lower reaches. Yearling (age 1+) rainbow/steelhead were most abundant in tributaries and upper mainstem reaches (Tables 2-6). Biomass of salmonids ranged from 0 to1,627 grams per $100 \mathrm{~m}^{2}$ (Tables 4 \& 5). Rainbow/steelhead parr (age 1+) yielded the greatest biomass at most sites.

\section{Snorkeling}

Snorkeling surveys were cut short in 2000 because of high turbid flows in June.

\begin{tabular}{|c|c|c|c|c|c|c|c|c|c|c|c|c|}
\hline \multirow{4}{*}{$\begin{array}{l}\begin{array}{l}\text { Stream } \\
\text { Reach }\end{array} \\
\text { Site Name }\end{array}$} & \multirow[b]{4}{*}{$\begin{array}{l}\text { (Mo/ } \\
\text { day) }\end{array}$} & \multirow{4}{*}{$\begin{array}{c}\begin{array}{c}\text { Site } \\
\text { Length }\end{array} \\
\text { (m) } \\
\end{array}$} & \multirow{4}{*}{$\begin{array}{c}\text { Mean } \\
\text { Width }\end{array}$} & \multirow{4}{*}{$\begin{array}{l}\text { Area } \\
\left(\mathbf{m}^{2}\right)\end{array}$} & \multicolumn{8}{|c|}{ Densities (\#/100 m²) } \\
\hline & & & & & \multicolumn{4}{|c|}{ Rainbow/steelhead } & \multirow[b]{3}{*}{$\begin{array}{c}\text { Other } \\
\text { Species }^{\text {a }}\end{array}$} & \multirow{2}{*}{\multicolumn{3}{|c|}{ Age/size }} \\
\hline & & & & & \multicolumn{4}{|c|}{ Age/size } & & & & \\
\hline & & & & & $\mathbf{0 +}$ & $1+$ & $\geq 8$ in & Total & & \multirow[t]{2}{*}{$\mathbf{0 +}$} & $1+$ & $\geq 8$ in \\
\hline \multicolumn{12}{|c|}{ Touchet River } & \\
\hline TR2 & $(6 / 7)$ & 75.0 & 17 & 1275 & 2.4 & 0.2 & 0.9 & 3.5 & & & & \\
\hline TR6 & $(6 / 22)$ & 93.0 & 20 & 1860 & 6.3 & 0.5 & 0.6 & 7.4 & BRT & 0.8 & $\mathbf{0}$ & 0.1 \\
\hline \multicolumn{13}{|c|}{ SF Touchet River } \\
\hline SFT-8 & $(6 / 7)$ & 100.0 & 7.7 & 770 & 3.0 & 0.8 & 2.5 & 6.3 & & & & \\
\hline SFT-9 & $(6 / 7)$ & 75.0 & 10.1 & 758 & 1.7 & 0.4 & 2.6 & 4.5 & BRT & & & 0.1 \\
\hline \multicolumn{13}{|c|}{ Walla Walla River } \\
\hline WW1 & $(6 / 30)$ & 134.6 & 8.1 & 1090 & 18.5 & 1.6 & 0.3 & 20.4 & MTW & 0.1 & & \\
\hline WW5 & $(6 / 23)$ & 93 & 17.2 & 1599.6 & 204 & 0.7 & 0.3 & 3.4 & & & & \\
\hline WW9 & $(\mathbf{5} / \mathbf{3 0})$ & 110 & 13.7 & 1507 & 205 & 0.2 & 0.3 & 3 & & & & \\
\hline WW10 & $(\mathbf{5} / \mathbf{3 0})$ & 149 & 13.1 & 1951.9 & 1.2 & $\mathbf{0}$ & 0 & 1.2 & & & & \\
\hline
\end{tabular}

\section{Non-Salmonid Species Abundance and Distribution}

Speckled dace and sculpins were the most common non-salmonids found at most of our sampling sites (Appendix F). Speckled dace generally did not exist at upper sites where water temperatures were relatively cool. A longnose dace was observed during electrofishing in Mill Creek. Torrent sculpin distribution was limited to mid and lower river sites, and these fish were usually found in low densities. Paiute and Margined sculpin are found throughout the basin except in the lower sections of the mainstem Walla Walla and Touchet rivers. Northern Pikeminnow are distributed in lower sections of tributaries and mainstem rivers. Tailed frogs/ tadpoles were found only in upper sites in cold, clean water. During our efforts we have generally found bull trout where tailed frogs were present, but we have also found tailed frogs in headwater areas where bull trout are not present. 


\section{Spawning Surveys}

\section{Steelhead}

Steelhead spawning surveys were conducted in Coppei Creek, Dry Creek, and Patit Creek in 2000 between April and late May (Table 7). Five steelhead redds were observed in the lower 2.5 miles of South Patit Creek. Four of five steelhead observed in South Patit Creek were wild and the other was unknown. The North Fork, South Fork and mainstem Coppei Creek produced a total of 31 steelhead redds in the 14.3 stream miles surveyed, averaging 2.2 redds per mile (Table 7). Three wild, two unknown, and one hatchery steelhead were observed in the Coppei system. In addition, a $435 \mathrm{~mm}$ hatchery female steelhead that was spawned out was captured in Coppei Creek on January 25, 2000. Five steelhead redds were observed in Dry Creek in 7.2 stream miles surveyed. Two steelhead redds were observed in North Fork Dry Creek. In Cottonwood Creek, one steelhead redd and three steelhead were observed. One steelhead was a dead wild female, one was a wild male, and one was unknown. Surveys in the North Fork, Wolf Fork, Robinson Fork, and South Fork Touchet Rivers were conducted by the Snake River Lab, Dayton.

Table 7. Steelhead spawning survey summary for some of the tributaries of the Walla Walla River in Washington State, 2000.

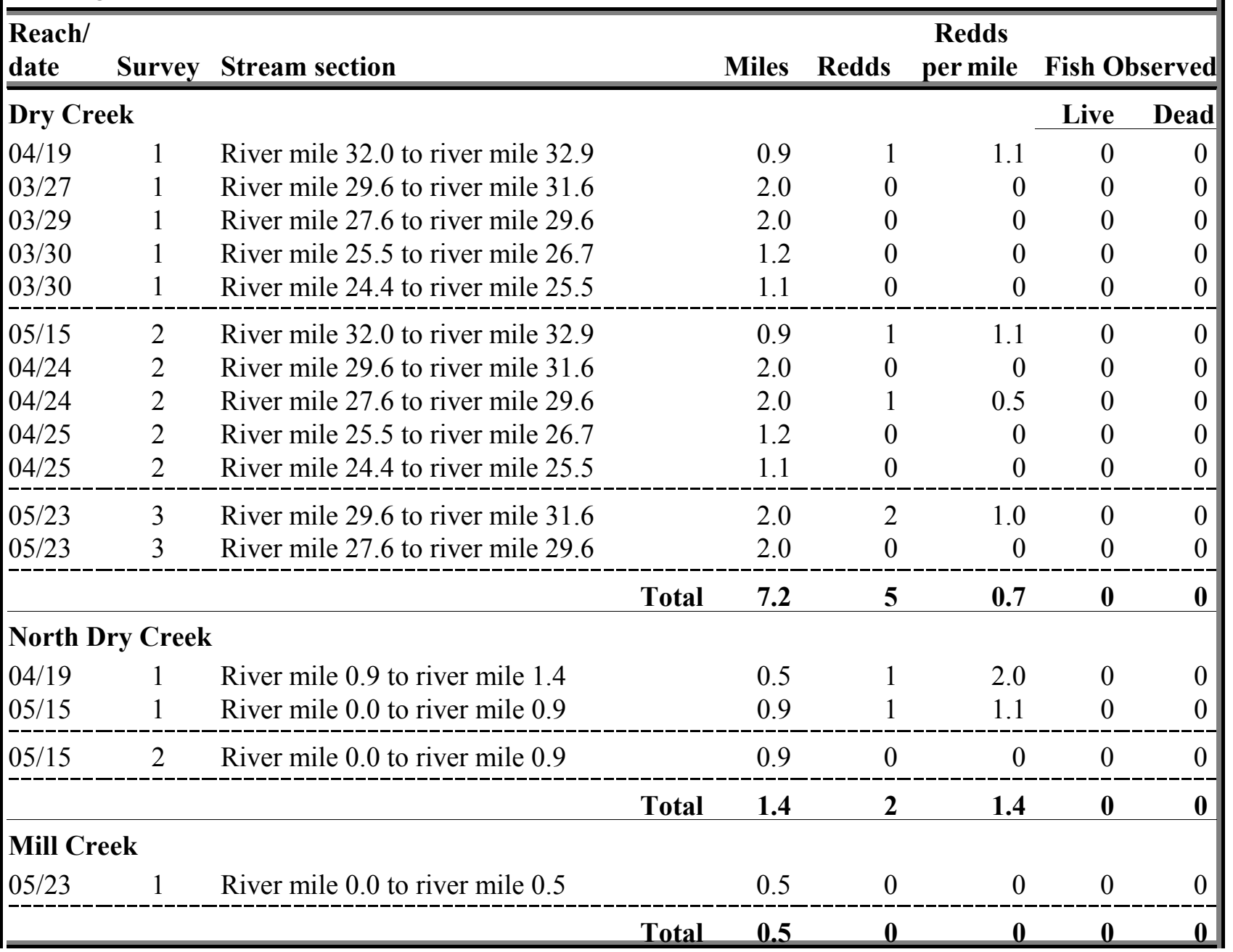


Table 7. (Continued) Steelhead spawning survey summary for some of the tributaries of the Walla Walla River in Washington State, 2000.

\begin{tabular}{|c|c|c|c|c|c|c|c|c|c|}
\hline $\begin{array}{l}\text { Reach/ } \\
\text { date }\end{array}$ & Survey & Stream section & & Miles & Redds & $\begin{array}{c}\text { Redds } \\
\text { per mile }\end{array}$ & Fish O & Dbservec & \\
\hline East Lit & tle Walla & Walla & & & & & Live & Dead & \\
\hline $03 / 14$ & 1 & River mile 0.6 to river mile 1.7 & & 1.1 & 0 & 0 & 2 & 0 & 0 \\
\hline $04 / 03$ & 2 & River mile 0.6 to river mile 1.7 & & 1.1 & 0 & 0 & 0 & 0 & 0 \\
\hline $04 / 25$ & 3 & River mile 0.6 to river mile 1.7 & & 1.1 & 0 & 0 & 0 & & 0 \\
\hline & & & Total & 1.1 & $\mathbf{0}$ & $\mathbf{0}$ & 2 & & $\mathbf{0}$ \\
\hline West Li & ttle Wall & a Walla & & & & & & & \\
\hline $03 / 14$ & 1 & River mile 3.0 to river mile 4.1 & & 1.1 & 0 & 0 & 0 & & 0 \\
\hline $04 / 03$ & 2 & River mile 3.0 to river mile 4.1 & & 1.1 & 1 & 0.9 & 0 & 0 & 0 \\
\hline $04 / 25$ & 3 & River mile 3.0 to river mile 4.1 & & 1.1 & 0 & 0 & 0 & 0 & 0 \\
\hline & & & Total & 1.1 & 1 & 0.9 & $\mathbf{0}$ & $\mathbf{0}$ & $\mathbf{0}$ \\
\hline Cottony & vood Cre & & & & & & & & \\
\hline $04 / 06$ & 1 & River mile 4.2 to river mile 5.3 & & 1.1 & 0 & 0 & 0 & & 0 \\
\hline $04 / 06$ & 1 & River mile 2.5 to river mile 4.2 & & 1.7 & 0 & 0 & 0 & & 0 \\
\hline $04 / 06$ & 1 & River mile 0.8 to river mile 2.5 & & 1.7 & 0 & 0 & 0 & 0 & 0 \\
\hline $04 / 25$ & 2 & River mile 4.2 to river mile 5.3 & & 1.1 & 0 & 0 & 0 & 0 & 0 \\
\hline $04 / 25$ & 2 & River mile 2.5 to river mile 4.2 & & 1.7 & 1 & 0.6 & 0 & & 0 \\
\hline $04 / 25$ & 2 & River mile 0.8 to river mile 2.5 & & 1.7 & 0 & 0 & 2 & 1 & 1 \\
\hline $05 / 15$ & 3 & River mile 4.2 to river mile 5.3 & & 1.1 & 0 & 0 & 0 & & 0 \\
\hline $05 / 15$ & 3 & River mile 2.5 to river mile 4.2 & & 1.7 & 0 & 0 & 0 & 0 & 0 \\
\hline $05 / 15$ & 3 & River mile 0.8 to river mile 2.5 & & 1.7 & 0 & 0 & 0 & 0 & 0 \\
\hline & & & Total & 4.5 & 1 & 0.2 & 2 & 1 & 1 \\
\hline Russell & Creek & & & & & & & & \\
\hline $04 / 06$ & 1 & River mile 6.7 to river mile 7.8 & & 1.1 & 0 & 0 & 0 & 0 & 0 \\
\hline $04 / 06$ & 1 & River mile 3.0 to river mile 5.8 & & 2.8 & 0 & 0 & 0 & 0 & 0 \\
\hline & & & Total & 3.9 & $\mathbf{0}$ & $\mathbf{0}$ & $\mathbf{0}$ & $\mathbf{0}$ & $\mathbf{0}$ \\
\hline Yellowh & lawk Cre & & & & & & & & \\
\hline $04 / 25$ & 1 & River mile 0.8 to river mile 2.7 & & 1.9 & 0 & 0 & 1 & 0 & 0 \\
\hline $05 / 22$ & 1 & River mile 7.1 to river mile 8.3 & & 1.2 & 0 & 0 & 0 & & 0 \\
\hline $05 / 16$ & 2 & River mile 0.8 to river mile 2.7 & & 1.5 & 1 & 0.7 & 0 & 0 & 0 \\
\hline & & & Total & 3.1 & 1 & 0.4 & 1 & & $\mathbf{0}$ \\
\hline
\end{tabular}




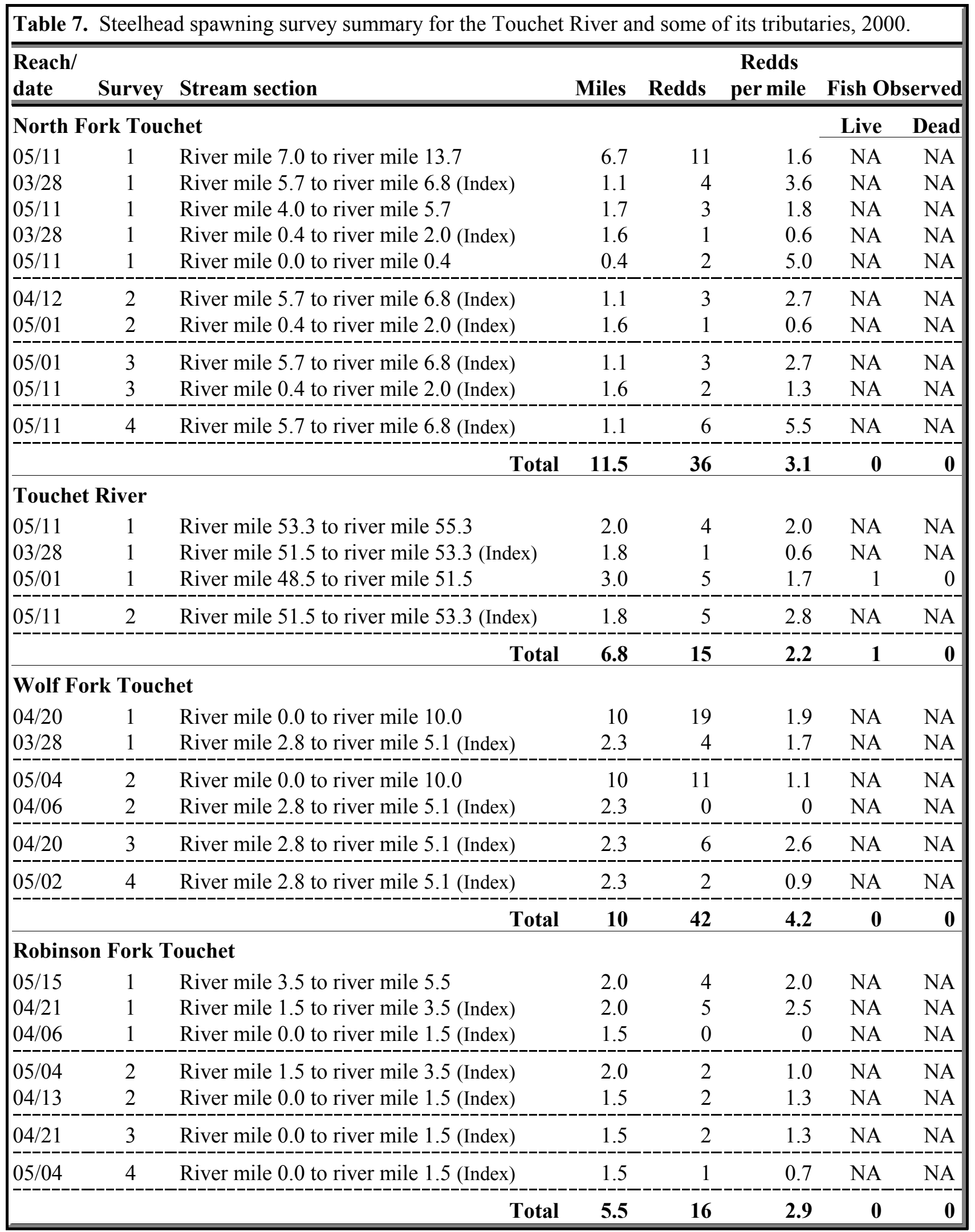


Table 7. (Continued) Steelhead spawning survey summary for the Touchet River and some of its tributaries, 2000.

\begin{tabular}{|c|c|c|c|c|c|c|c|}
\hline $\begin{array}{l}\text { Reach/ } \\
\text { date }\end{array}$ & Survey & Stream section & Miles & Redds & $\begin{array}{c}\text { Redds } \\
\text { per mile }\end{array}$ & \multicolumn{2}{|c|}{ Fish Observed } \\
\hline \multicolumn{6}{|c|}{ South Fork Touchet } & Live & Dead \\
\hline $05 / 02$ & 1 & River mile 12.7 to river mile 14.7 & 2.0 & 6 & 3.0 & NA & NA \\
\hline $05 / 02$ & 1 & River mile 9.7 to river mile 11.7 & 2.0 & 7 & 3.5 & NA & NA \\
\hline $05 / 02$ & 1 & River mile 7.7 to river mile 8.7 & 1.0 & 2 & 2 & NA & NA \\
\hline $03 / 27$ & 1 & River mile 5.0 to river mile 7.0 (Index) & 2.0 & 3 & 1.5 & NA & NA \\
\hline $03 / 27$ & 1 & River mile 2.0 to river mile 4.0 (Index) & 2.0 & 2 & 1 & NA & NA \\
\hline $04 / 10$ & 2 & River mile 5.0 to river mile 7.0 (Index) & 2.0 & 3 & 1.5 & NA & NA \\
\hline $04 / 10$ & 2 & River mile 2.0 to river mile 4.0 (Index) & 2.0 & 6 & 3.0 & NA & NA \\
\hline $05 / 01$ & 3 & River mile 5.0 to river mile 7.0 (Index) & 2.0 & 2 & 1.0 & NA & NA \\
\hline \multirow[t]{2}{*}{$05 / 01$} & 3 & River mile 2.0 to river mile 4.0 (Index) & 2.0 & 1 & 0.5 & NA & NA \\
\hline & & Total & 11.0 & 32 & 2.9 & $\mathbf{0}$ & $\mathbf{0}$ \\
\hline \multicolumn{8}{|c|}{ Lewis Creek } \\
\hline $04 / 25$ & 1 & River mile 1.2 to river mile 2.1 & 0.9 & 0 & 0 & 0 & 0 \\
\hline \multirow[t]{2}{*}{$04 / 25$} & 1 & River mile 0.0 to river mile 1.2 & 1.2 & 0 & 0 & 0 & 0 \\
\hline & & Total & 2.1 & $\mathbf{0}$ & $\mathbf{0}$ & $\mathbf{0}$ & $\mathbf{0}$ \\
\hline \multicolumn{8}{|c|}{ Jim Creek } \\
\hline \multirow[t]{2}{*}{ 05/01 } & 1 & River mile 0.0 to river mile 0.3 & 0.3 & 0 & 0 & 0 & 0 \\
\hline & & Total & 0.3 & $\mathbf{0}$ & $\mathbf{0}$ & $\mathbf{0}$ & $\mathbf{0}$ \\
\hline \multicolumn{8}{|c|}{ South Patit Creek } \\
\hline $05 / 01$ & 1 & River mile 2.1 to river mile 3.6 & 1.5 & 3 & 2.0 & 5 & 0 \\
\hline $05 / 01$ & 1 & River mile 1.1 to river mile 2.1 & 1.0 & 1 & 1.0 & 0 & 0 \\
\hline $05 / 19$ & 1 & River mile 2.1 to river mile 3.6 & 1.5 & 1 & 0.7 & 0 & 0 \\
\hline \multirow[t]{2}{*}{$05 / 19$} & 1 & River mile 1.1 to river mile 2.1 & 1.0 & 0 & 0 & 0 & 0 \\
\hline & & Total & 2.5 & 5 & 2 & 5 & $\mathbf{0}$ \\
\hline \multicolumn{8}{|c|}{ North Coppei Creek } \\
\hline $03 / 29$ & 1 & River mile 3.6 to river mile 5.1 & 1.5 & 0 & 0 & 0 & 0 \\
\hline $03 / 29$ & 1 & River mile 2.5 to river mile 3.6 & 1.1 & 0 & 0 & 0 & 0 \\
\hline $03 / 22$ & 1 & River mile 0.8 to river mile 1.4 & 0.6 & 2 & 3.3 & 0 & 0 \\
\hline $03 / 22$ & 1 & River mile 0.0 to river mile 0.8 & 0.8 & 1 & 1.3 & 1 & 0 \\
\hline $04 / 18$ & 2 & River mile 3.6 to river mile 5.1 & 1.5 & 0 & 0 & 0 & 0 \\
\hline $04 / 18$ & 2 & River mile 2.5 to river mile 3.6 & 1.1 & 0 & 0 & 0 & 0 \\
\hline $04 / 18$ & 2 & River mile 0.8 to river mile 1.4 & 0.6 & 0 & 0 & 0 & 0 \\
\hline $04 / 18$ & 2 & River mile 0.0 to river mile 0.8 & 0.8 & 0 & 0 & 0 & 0 \\
\hline $05 / 10$ & 2 & River mile 3.6 to river mile 5.1 & 1.5 & 0 & 0 & 0 & 0 \\
\hline $05 / 10$ & 2 & River mile 2.5 to river mile 3.6 & 1.1 & 0 & 0 & 0 & 0 \\
\hline $05 / 10$ & 2 & River mile 0.8 to river mile 1.4 & 0.6 & 0 & 0 & 0 & 0 \\
\hline \multirow[t]{2}{*}{ 05/10 } & 2 & River mile 0.0 to river mile 0.8 & 0.8 & 1 & 1.3 & 0 & 0 \\
\hline & & Total & 4.0 & 4 & 1.0 & 1 & $\mathbf{0}$ \\
\hline
\end{tabular}


Table 7. (Continued) Steelhead spawning survey summary for the Touchet River and some of its tributaries, 2000.

\begin{tabular}{|c|c|c|c|c|c|c|c|c|}
\hline $\begin{array}{l}\text { Reach/ } \\
\text { date }\end{array}$ & Survey & Stream section & & Miles & Redds & $\begin{array}{c}\text { Redds } \\
\text { per mile }\end{array}$ & Fish $\mathbf{O}$ & erved \\
\hline South & oppei C & eek & & & & & Live & Dead \\
\hline $03 / 30$ & 1 & River mile 4.3 to river mile 4.9 & & 0.6 & 1 & 1.7 & 0 & 0 \\
\hline $03 / 21$ & 1 & River mile 3.2 to river mile 4.3 & & 1.1 & 1 & 0.9 & 0 & 0 \\
\hline $03 / 21$ & 1 & River mile 2.1 to river mile 3.2 & & 1.1 & 1 & 0.9 & 0 & 0 \\
\hline $03 / 21$ & 1 & River mile 0.0 to river mile 2.1 & & 2.1 & 0 & 0 & 0 & 0 \\
\hline $04 / 18$ & 2 & River mile 4.3 to river mile 4.9 & & 0.6 & 0 & 0 & 0 & 0 \\
\hline $04 / 18$ & 2 & River mile 3.2 to river mile 4.3 & & 1.1 & 3 & 2.7 & 0 & 0 \\
\hline $04 / 18$ & 2 & River mile 2.1 to river mile 3.2 & & 1.1 & 3 & 2.7 & 0 & 0 \\
\hline $04 / 18$ & 2 & River mile 0.0 to river mile 2.1 & & 2.1 & 2 & 1.0 & 0 & 0 \\
\hline $05 / 10$ & 3 & River mile 3.2 to river mile 4.3 & & 1.1 & 0 & 0 & 0 & 0 \\
\hline $05 / 10$ & 3 & River mile 2.1 to river mile 3.2 & & 1.1 & 0 & 0 & 0 & 0 \\
\hline $05 / 10$ & 3 & River mile 0.0 to river mile 2.1 & & 2.1 & 5 & 2.4 & 0 & 0 \\
\hline & & & Total & 4.9 & 16 & 3.3 & $\mathbf{0}$ & $\mathbf{0}$ \\
\hline Coppei & Creek & & & & & & & \\
\hline $03 / 22$ & 1 & River mile 6.1 to river mile 7.2 & & 1.1 & 0 & 0 & 0 & 0 \\
\hline $03 / 27$ & 1 & River mile 4.6 to river mile 6.1 & & 1.5 & 2 & 1.3 & 0 & 0 \\
\hline $03 / 27$ & 1 & River mile 3.1 to river mile 4.6 & & 1.5 & 0 & 0 & 4 & 0 \\
\hline $03 / 30$ & 1 & River mile 1.8 to river mile 3.1 & & 1.3 & 0 & 0 & 0 & 0 \\
\hline $04 / 07$ & 2 & River mile 6.1 to river mile 7.2 & & 1.1 & 5 & 4.5 & 1 & 0 \\
\hline $04 / 19$ & 2 & River mile 4.6 to river mile 6.1 & & 1.5 & 0 & 0 & 0 & 0 \\
\hline $04 / 04$ & 2 & River mile 3.1 to river mile 4.6 & & 1.5 & 0 & 0 & 0 & 0 \\
\hline $04 / 19$ & 2 & River mile 1.8 to river mile 3.1 & & 1.3 & 0 & 0 & 0 & 0 \\
\hline $04 / 19$ & 3 & River mile 6.1 to river mile 7.2 & & 1.1 & 2 & 1.8 & 0 & 0 \\
\hline $05 / 15$ & 3 & River mile 4.6 to river mile 6.1 & & 1.5 & 1 & 0.7 & 0 & 0 \\
\hline $05 / 16$ & 3 & River mile 3.1 to river mile 4.6 & & 1.5 & 0 & 0 & 0 & 0 \\
\hline $05 / 15$ & 3 & River mile 1.8 to river mile 3.1 & & 1.3 & 0 & 0 & 0 & 0 \\
\hline $05 / 16$ & 4 & River mile 6.1 to river mile 7.2 & & 1.1 & 1 & 0.9 & 0 & 0 \\
\hline & & & Total & 5.4 & 11 & 2.0 & 5 & $\mathbf{0}$ \\
\hline
\end{tabular}


Bull trout spawning surveys were conducted in the upper Wolf Fork Touchet in 2000 (Table 8). Water temperatures in the Wolf Fork during bull trout spawning season were generally in the low to mid 40's (EF). A total of 48 bull trout and 64 redds were observed between river mile 7.3 and river mile 12.8. Redd counts in the Wolf Fork from 1990-2000 are presented in Figure 12.

In 2000, we also surveyed the Burnt Fork Touchet from its headwaters downstream to determine bull trout spawning distribution (Table 9.). Spawning distribution was limited to the lower 2.5 miles of the stream. A total of 4 redds and 6 bull trout were observed .

Table 8. Bull trout spawning survey summary for the Wolf Fork of the Touchet River, 2000.

\begin{tabular}{|c|c|c|c|c|c|}
\hline Reach/date & Survey & Stream section & Miles & Redds & $\begin{array}{c}\text { Redds } \\
\text { per mile }\end{array}$ \\
\hline $9 / 8$ & 1 & (A) River mile 11.3 to river mile 12.8 & 1.5 & 1 & 0.7 \\
\hline $9 / 8$ & 1 & (B) River mile 10.3 to river mile 11.3 & 1.0 & 7 & 7 \\
\hline $9 / 8$ & 1 & (C) River mile 9.6 to river mile 10.3 & 0.7 & 15 & 21.4 \\
\hline $9 / 8$ & 1 & (D) River mile 8.7 to river mile 9.6 & 0.9 & 3 & 3.3 \\
\hline $9 / 21$ & 2 & (A) River mile 11.3 to river mile 12.8 & 1.5 & 1 & 0.7 \\
\hline $9 / 21$ & 2 & (B) River mile 10.3 to river mile 11.3 & 1.0 & 7 & 7 \\
\hline $9 / 21$ & 2 & (C) River mile 9.6 to river mile 10.3 & 0.7 & 11 & 15.7 \\
\hline $9 / 21$ & 2 & (D) River mile 8.7 to river mile 9.6 & 0.9 & 3 & 3.3 \\
\hline $9 / 21$ & 2 & (E) River mile 7.3 to river mile 8.7 & 1.4 & 1 & 0.7 \\
\hline $10 / 10$ & 3 & (A) River mile 11.3 to river mile 12.8 & 1.5 & 1 & 0.7 \\
\hline $10 / 10$ & 3 & (B) River mile 10.3 to river mile 11.3 & 1.0 & 3 & 3 \\
\hline $10 / 10$ & 3 & (C) River mile 9.6 to river mile 10.3 & 0.7 & 4 & 5.7 \\
\hline $10 / 10$ & 3 & (D) River mile 8.7 to river mile 9.6 & 0.9 & 1 & 1.1 \\
\hline $10 / 10$ & 3 & (E) River mile 7.3 to river mile 8.7 & 1.4 & 1 & 0.7 \\
\hline $10 / 23$ & 4 & (B) River mile 10.3 to river mile 11.3 & 1.0 & 0 & 0 \\
\hline $10 / 23$ & 4 & (C) River mile 9.6 to river mile 10.3 & 0.7 & 3 & 4.3 \\
\hline $10 / 23$ & 4 & (D) River mile 8.7 to river mile 9.6 & 0.9 & 0 & 0 \\
\hline \multirow[t]{2}{*}{$10 / 23$} & 4 & (E) River mile 7.3 to river mile 8.7 & 1.4 & 2 & 1.4 \\
\hline & & & 5.5 & 64 & 11.6 \\
\hline
\end{tabular}

\begin{tabular}{|c|c|c|c|c|c|}
\hline Reach/date & Survey & Stream section & Miles & Redds & $\begin{array}{c}\text { Redds } \\
\text { per mile }\end{array}$ \\
\hline $9 / 12$ & 1 & River mile 3.0 to river mile 1.5 & 1.5 & 1 & 0.67 \\
\hline $9 / 27$ & 2 & River mile 3.0 to river mile 2.0 & 1.0 & 0 & 0 \\
\hline $10 / 16$ & 3 & River mile 3.0 to river mile 2.0 & 1.0 & 0 & 0 \\
\hline \multirow[t]{2}{*}{$10 / 16$} & 3 & River mile 2.0 to river mile 0.0 & 2.0 & 3 & 1.5 \\
\hline & & & 3.0 & 4 & 1.3 \\
\hline
\end{tabular}




\section{Total Bull Trout Redds/Year \\ Wolf Fork Touchet}

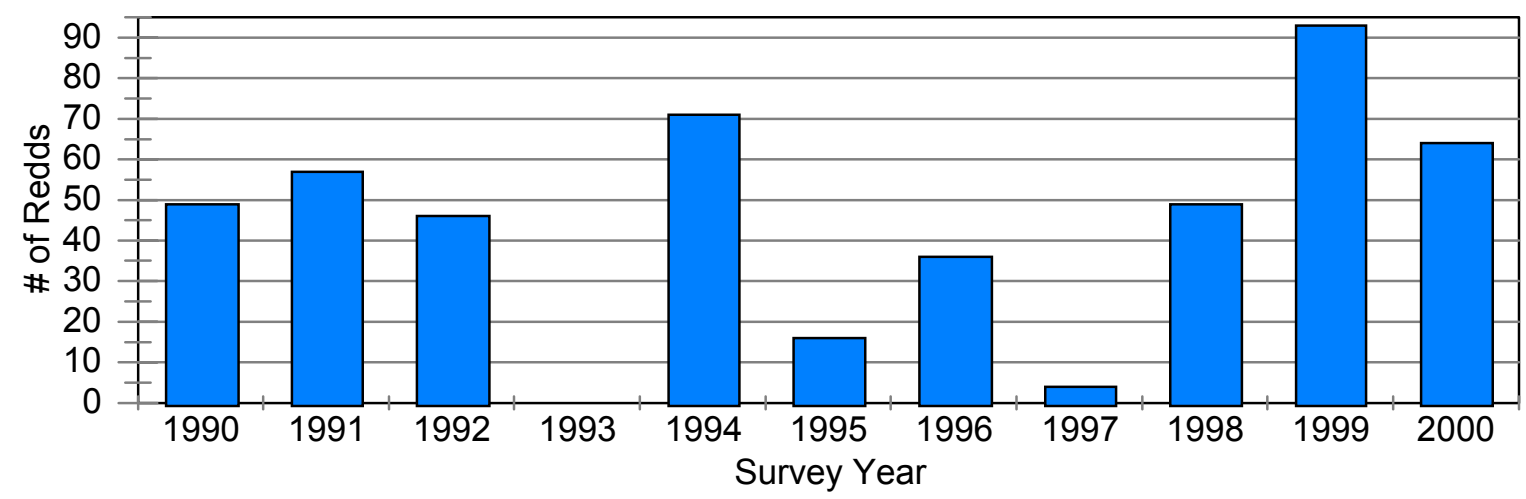

\begin{tabular}{|c|c|c|c|c|c|c|c|}
\hline \multirow[b]{3}{*}{ Year } & \multicolumn{6}{|c|}{ Reach Surveyed ${ }^{a}$} & \multirow[b]{3}{*}{$\begin{array}{l}\text { Total } \\
\text { Redds }\end{array}$} \\
\hline & $\mathbf{A}$ & B & C & D & $\mathbf{E}$ & $\mathbf{F}$ & \\
\hline & $\begin{array}{c}\text { River Mile } \\
12.8-11.3\end{array}$ & $\begin{array}{c}\text { River Mile } \\
11.3-10.3\end{array}$ & $\begin{array}{c}\text { River Mile } \\
10.3-9.6\end{array}$ & $\begin{array}{c}\text { River Mile } \\
9.6-8.7\end{array}$ & $\begin{array}{c}\text { River Mile } \\
8.7-7.3\end{array}$ & $\begin{array}{c}\text { River Mile } \\
7.3 \text { - } 6.8\end{array}$ & \\
\hline 1990 & & 18 & 31 & & & & 49 \\
\hline 1991 & & 20 & 37 & & & & 57 \\
\hline 1992 & & & $\overline{46}$ & & & & 46 \\
\hline $1993^{b}$ & & & & & & & 0 \\
\hline 1994 & & & 71 & & & & 71 \\
\hline 1995 & & & 16 & & & & 16 \\
\hline 1996 & & & 36 & & & & 36 \\
\hline $1997^{\mathrm{c}}$ & & & & & 4 & & 4 \\
\hline 1998 & 11 & 7 & 18 & 12 & 0 & & 48 \\
\hline 1999 & 32 & 14 & 34 & 11 & 2 & & 93 \\
\hline 2000 & 3 & 17 & 33 & 7 & 4 & & 64 \\
\hline
\end{tabular}

a. A: RM 11.3 = USFS boundary, B: RM 10.3 = Tate Cr., C: RM 9.6 = Newby's ford, D: RM 8.7 = second bridge down, E: RM 7.3 = Whitney Cr., F: RM 6.8 = County bridge.

b. No survey.

c. One survey done late in October and too far down stream.

Figure 12. Bull trout spawning survey summary for the Wolf Fork of the Touchet River, 1990-2000.

In 2000, WDFW participated with the USFS in a bull trout spawning survey of the North Fork Touchet River. Thirty-six bull trout and 43 redds were observed between Bluewood Creek and Spangler Creek. The Forest Service has been conducting bull trout spawning surveys since 1994 on the North Fork Touchet, during which time redd numbers have steadily increased (Figure 13). 


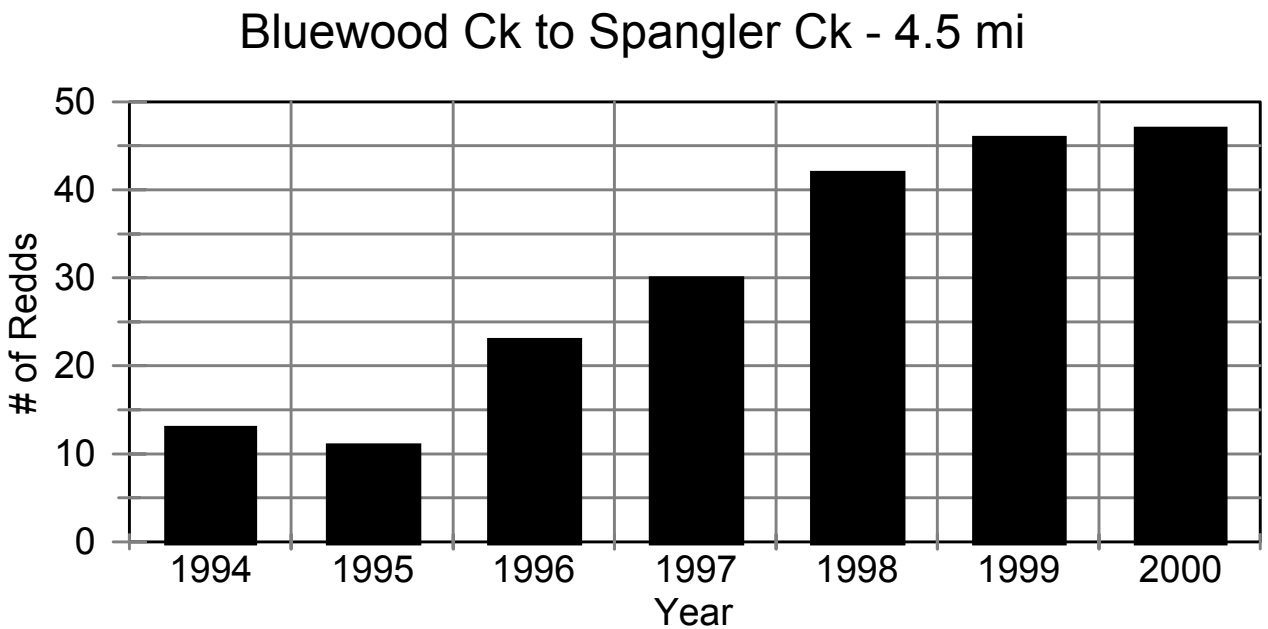

Figure 13. Bull trout redd counts for the North Fork Touchet, 1994-2000

\section{Genetic Sampling}

Fin clips were collected from a total of 103 bull trout during the 2000 season; 14 adult bull trout were collected from the trap at Nursery Bridge in Oregon, 21 adult bull trout from the trap on the Touchet River in Dayton, Washington, and 50 adult bull trout were collected at the Mill Creek watershed intake dam in Oregon. Eighteen other bull trout samples were collected from adults and juveniles during electrofishing surveys.

Fin clip or opercle punch tissue samples were collected from 45 adult steelhead in the Walla Walla basin during the 2000 season; 30 from the trap at Dayton Touchet River, 11 from WDFW trap on Yellowhawk Creek, 2 from Coppei Creek and 2 from Cottonwood Creek..

Tissue samples were collected for 180 juvenile rainbow/steelhead trout using electrofishing equipment; 60 fish from the North Fork Touchet, 60 fish from the Robinson Fork Touchet, and 60 fish from Coppei Creek. Fish were collected only if they were approximately age $1+$ or older $(\geq 80$ $\mathrm{mm})$. We collected no more than 6 fish each from several sites to minimize the chances of collecting siblings.

Tissue samples were collected from a total of 38 mountain whitefish in the Walla Walla basin in 2000; 20 from Mill Creek at the watershed intake dam, 15 from the Touchet River at the Dayton trap, and 3 from the Walla Walla River below Burlingame dam during electrofishing sampling.

Some of the genetic samples were sent to the WDFW Genetics Stock Identification Lab for DNA analysis; others are being held at the fish management office in Dayton. 
Armour, C. L. and W. S. Platts 1983. Field methods and statistical analyses for monitoring small salmonid streams. US Fish and Wildlife Service. FWS/OBS-83/33. 200 pages.

Bjornn, T. and D. Reiser, 1991. Habitat Requirements of Salmonids in Streams. In Influences of Forest and Rangeland on Salmonid Fishes and their Habitats. W. Meehan (editor). Am. Fish. Soc. Special Pub. 19.

Collier, M., R. H. Webb, and J. C. Schmidt 1996. Dams and rivers: primer on the downstream effects of dams. US Geological Survey, Circular 1126. Tucson. 94 pages.

Knutson, K. L.,S. Jackson, T. Lovgren, M.. Hunter, and D. McDonald 1992 Washington Rivers Information System: resident and anadromous fish data, 1:100,000 scale update. Washington Department of Wildlife, Olympia. 25 pages plus extensive appendices.

Mendel, G., V. Naef, and D. Karl. 1999. Assessment of Salmonid Fishes and their Habitat Condiditons in the Walla Walla River Basin - 1998 Annual Report. Report to BPA. Project 9820. Report \# FPA 99-01. 94 pages.

Mongillo, P. E. 1993. The distribution and status of bull trout/dolly varden in Washington State. Washington Department of Wildlife, Olympia. 45 pages.

Nielson, R. S. 1950. Survey of the Columbia River and its Tributaries, Part 5. US Fish and Wildlife Service, Scientific Report, No. 38. 41 pages.

Olsen, J. B., J. K. Wenburg, and P. Benson, 1996. Semi-automated multilocus genotyping of Pacific salmon (Oncorhynchus spp.) Using microsatellites. Molecular. Marine Biol. and Biotech. 5:259272.

Pacific Groundwater Group. 1995. Draft Initial Watershed Assessment, Water Resources Inventory Area 32, Walla Walla Watershed - Open file Technical Report 95-11. Seattle, 47 pages, plus appendices.

Pirtle, R. 1957. Field studies to establish the size and timing of runs of anadromous species of fish in the Columbia and Snake rivers and their distribution above the confluence of the Snake River. Final Report to the US Army Corps of Engineers, Idaho Fish and Game, Boise. 49pp plus appendices.

Platts, W. S., W. F. Megahan, and G.W. Minshall. 1983. Methods for evaluating stream, riparian, and biotic conditions. USDA Forest Service. Ogden. GTR.INT-138. 70 pages.

US Army Corps of Engineers (ACOE) 1992. Walla Walla River Basin, Oregon and Washington: reconnaissance report. Walla Walla. 43 pages, plus extensive appendices. 
US Army Corps of Engineers (ACOE) 1997. Walla Walla River Watershed, Oregon and Washington: reconnaissance report. Walla Walla. 78 pages, plus extensive appendices.

Van Deventer, J. S. and Platts, W. S. 1983. Sampling and estimating fish populations from streams. Trans. N. Am. Wildl. And Nat. Res. Conf. 48: 349-354.

Walla Walla Daily Journal. 1884. Young carp: the arrival of a government fish car at Walla Walla.

Washington Department of Fisheries (WDF) and Washington Department of Wildlife (WDW). 1993. 1992 Washington State salmon and steelhead stock inventory: Columbia River stocks. Olympia, WA. 579 pages.

Wydoski, R.S. and R. R. Whitney 1979. Inland fishes of Washington. University of Washington Press, Seattle. 220 pages. 


\begin{tabular}{|c|c|c|c|c|c|}
\hline Reach & Site \# & $\mathbf{R M}^{\mathbf{a}}$ & Location & $\begin{array}{l}\text { Sample } \\
\text { Type }^{\mathrm{b}}\end{array}$ & Comments \\
\hline $\begin{array}{l}\text { NF Touchet } \\
\text { River }\end{array}$ & $\begin{array}{l}\text { NFT-1 } \\
\text { NFT-2 } \\
\text { NFT-3 } \\
\text { NFT-4 } \\
\text { NFT-5 } \\
\text { NFT-6 } \\
\text { NFT-7 } \\
\text { NFT-8 } \\
\text { NFT-9 } \\
\text { NFT-10 } \\
\text { NFT-11 } \\
\text { NFT-12 } \\
\text { NFT-13 } \\
\text { NFT-14 } \\
\text { NFT-15 } \\
\text { NFT-16 } \\
\text { NFT-17 }\end{array}$ & $\begin{array}{r}18.4 \\
17.0 \\
16.6 \\
16.0 \\
15.6 \\
15.3 \\
13.7 \\
12.7 \\
10.7 \\
9.3 \\
7.5 \\
5.7 \\
4.4 \\
3.1 \\
2.1 \\
1.6 \\
0\end{array}$ & 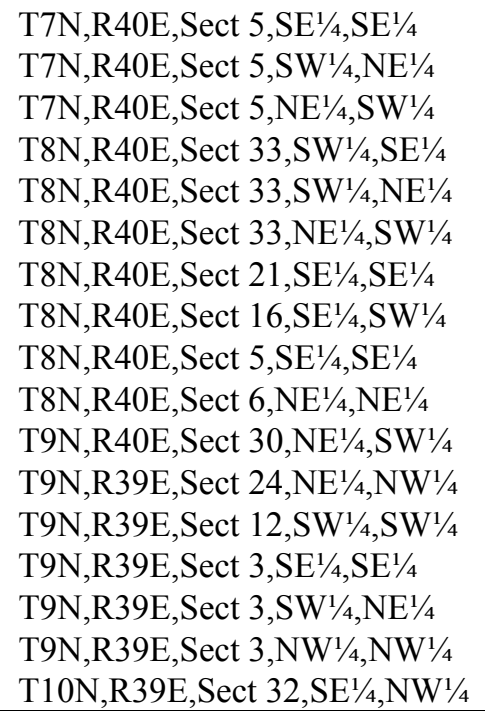 & $\begin{array}{l}\text { EQ,T } \\
\text { EL } \\
\text { EL,EQ } \\
\text { EL } \\
\text { EL } \\
\text { EQ } \\
\text { T,F } \\
\text { EQ } \\
\text { EQ } \\
\text { EQ } \\
\text { EQ,T,F } \\
\text { EQ } \\
\text { EQ } \\
\text { EQ } \\
\text { EQ } \\
\text { EQ,T,F } \\
\text { EQ }\end{array}$ & $\begin{array}{l}\text { Bluewood culvert } \\
3.3 \mathrm{mi} \text { above Spangler } \\
3.0 \mathrm{mi} \text { above Spangler turn } \\
2.3 \mathrm{mi} \text { above Spangler turn } \\
1.8 \mathrm{mi} \text { above Spangler turn } \\
\text { 1.5 mi above Spangler turn } \\
\text { Spangler mouth } \\
\text { Hompegg Falls } \\
\text { Just below Lewis Ck mouth } \\
\text { blue mailbox } \\
\text { Jim Ck mouth } \\
\text { 1.6 mi above Wolf Fk turn } \\
0.3 \text { mi above Wolf Fk turn } \\
\text { Orchard } \\
\text { Vernon Rd } \\
\text { Above Baileysburg } \\
\text { Just above confluence }\end{array}$ \\
\hline Corral Ck & $\begin{array}{l}\mathrm{CC}-1 \\
\mathrm{CC}-2 \\
\mathrm{CC}-3\end{array}$ & $\begin{array}{l}0.6 \\
0.2 \\
0.1 \\
\end{array}$ & $\begin{array}{l}\text { T7N,R40E,Sect 17,NW1/4,SW1/4 } \\
\text { T7N,R40E,Sect 18,NE1/4,NE1/4 } \\
\text { T7N,R40E,Sect 7,SE1/4,SE1/4 }\end{array}$ & $\begin{array}{l}\text { EL } \\
\text { EL } \\
\text { EL,F }\end{array}$ & $\begin{array}{l}2^{\text {nd }} \text { stream crossing } \\
5 \text { min above } 1^{\text {st }} \text { site } \\
1^{\text {st }} \text { stream crossing }\end{array}$ \\
\hline Spangler $\mathrm{Ck}$ & $\begin{array}{l}\text { SC-1 } \\
\text { SC-2 } \\
\text { SC-3 } \\
\text { SC-4 } \\
\text { SC-5 } \\
\text { SC-6 }\end{array}$ & $\begin{array}{l}1.5 \\
1.2 \\
1.2 \\
1.0 \\
0.3 \\
0.2\end{array}$ & $\begin{array}{l}\text { T8N,R40E,Sect 34,NE } 1 / 4, \mathrm{NW}^{1 / 4} / 4 \\
\text { T8N,R40E,Sect 34,NE1/4,NW1/4 } \\
\text { T8N,R40E,Sect 34,NE1/4,NW1/4 } \\
\text { T8N,R40E,Sect 27,SE1/4,SW1/4 } \\
\text { T8N,R40E,Sect 27,NW1/4,NW1/4 } \\
\text { T8N,R40E,Sect 28,NE1/4,NE1/4 }\end{array}$ & $\begin{array}{l}\text { EQ } \\
\text { EQ } \\
\text { EQ } \\
\text { EQ } \\
\text { EQ } \\
\text { EQ,T,F }\end{array}$ & $\begin{array}{l}\text { Top site } \\
\text { Site } 3 \\
\text { Site } 2 \\
1 \text { mi above Spangler mouth } \\
0.3 \text { mi above Spangler mouth } \\
0.2 \text { mi above Spangler mouth }\end{array}$ \\
\hline Patrick Sp Ck & PSC-1 & 0.1 & T8N,R40E,Sect 27,NW1/4,SE1/4 & EL & $\sim 100$ yards above mouth \\
\hline Lewis Ck & $\begin{array}{l}\text { LC-1 } \\
\text { LC-2 } \\
\text { LC-3 } \\
\text { LC-4 } \\
\text { LC-5 } \\
\text { LC-6 } \\
\text { LC-7 } \\
\text { LC-8 } \\
\text { LC-9 } \\
\text { LC-10 } \\
\text { LC-11 } \\
\text { LC-12 }\end{array}$ & $\begin{array}{l}3.8 \\
3.5 \\
3.1 \\
2.8 \\
2.4 \\
1.9 \\
1.7 \\
1.6 \\
1.4 \\
0.4 \\
0.2 \\
0.1 \\
\end{array}$ & 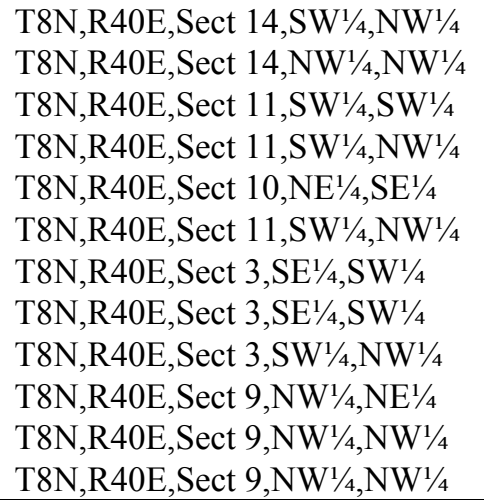 & $\begin{array}{l}\text { EQ } \\
\text { EQ } \\
\text { EQ } \\
\text { EQ } \\
\text { EQ } \\
\text { EQ } \\
\text { EQ } \\
\text { EQ } \\
\text { EQ } \\
\text { EQ } \\
\text { EQ } \\
\text { EQ,T,F }\end{array}$ & $\begin{array}{l}\text { Top/Headwaters } \\
0.3 \mathrm{mi} \text { down from top } \\
0.7 \mathrm{mi} \text { down from top } \\
1.7 \mathrm{mi} \text { above FS line } \\
1.3 \mathrm{mi} \text { above FS line } \\
0.8 \mathrm{mi} \text { above FS line } \\
0.7 \mathrm{mi} \text { above FS line } \\
0.5 \mathrm{mi} \text { above FS line } \\
0.2 \mathrm{mi} \text { above FS line } \\
1^{\text {st }} \text { bridge } \\
0.1 \mathrm{mi} \text { above NF Touchet Rd } \\
\text { At NF Touchet } \mathrm{R} \text { bridge }\end{array}$ \\
\hline Jim Creek & $\mathrm{JC}-1$ & 0.1 & T9N,R40E,Sect30,NE1/4,NE1/4 & $\mathrm{T}, \mathrm{F}$ & Below culvert \\
\hline Hatley Gulch & HG-1 & 0.7 & T9N,R39E,Sect 2,SE1/4,SE1/4 & EL & Hatley Gulch Rd \\
\hline Johnson & JH-1 & 1.0 & T10N,R39E,Sect 18,SE1/4,NW1/4 & EL & Eager Rd \\
\hline \multicolumn{6}{|c|}{$\begin{array}{ll} & \text { River mile. } \\
\text { b } & \text { EQ - Quantitative Electrofishing (density estimates); EL - Qualitative electrofishing; S - Snorkel; T - } \\
& \text { Temperature; F - Flow; W - Water Quality; G - Flow gauge. } \\
\text { c Index discharge sites. } \\
\text { d }\end{array}$} \\
\hline
\end{tabular}




\begin{tabular}{|c|c|c|c|c|c|}
\hline Reach & Site \# & $\mathbf{R M}^{\mathbf{a}}$ & Location & $\begin{array}{l}\text { Sample } \\
\text { Type }^{\mathrm{b}}\end{array}$ & Comments \\
\hline Davis Hollow & DH-1 & 0.3 & T10N,R39E,Sect 32,SE1/4,NE1/4 & EL & Baileysburg \\
\hline Cougar Canyon & $\mathrm{CC}-1$ & 0.1 & $\mathrm{~T} 10 \mathrm{~N}, \mathrm{R} 40 \mathrm{E}, \mathrm{Sect} 20, \mathrm{SW}^{1} / 4, \mathrm{SE}^{1} / 4$ & EL & Up Cougar Canyon Rd \\
\hline Mustard Hollow & MH-1 & 0.5 & T10N,R39E,Sect29,NW1/4,SE1/4 & EL & By cemetary \\
\hline Wolf Fork & $\begin{array}{l}\text { WF-1 } \\
\text { WF-2 } \\
\text { WF-3 } \\
\text { WF-4 } \\
\text { WF-5 } \\
\text { WF-6 } \\
\text { WF-7 } \\
\text { WF-8 } \\
\text { WF-9 } \\
\text { WF-10 } \\
\text { WF-11 } \\
\text { WF-12 }\end{array}$ & $\begin{array}{r}13.2 \\
12.7 \\
11.8 \\
11.1 \\
10.0 \\
7.6 \\
6.6 \\
5.2 \\
4.0 \\
3.2 \\
1.7 \\
0\end{array}$ & 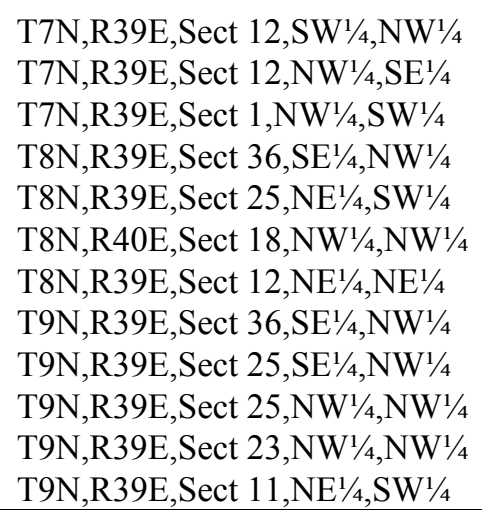 & $\begin{array}{l}\mathrm{EL}^{\mathrm{d}} \\
\mathrm{EQ}^{\mathrm{d}} \\
\mathrm{EQ}^{\mathrm{d}} \\
\mathrm{EQ}^{\mathrm{d}} \\
\mathrm{T}, \mathrm{F}, \mathrm{W}, \mathrm{EQ}^{\mathrm{d}} \\
\mathrm{EQ}^{\mathrm{d}} \\
\mathrm{EQ}^{\mathrm{d}} \\
\mathrm{EQ}^{\mathrm{d}} \\
\mathrm{T}, \mathrm{F}^{\mathrm{c}} \\
\mathrm{EQ}^{\mathrm{d}} \\
\mathrm{F}^{\mathrm{c}}, \mathrm{T}, \mathrm{EQ}^{\mathrm{d}} \\
\mathrm{EQ}^{\mathrm{d}}\end{array}$ & $\begin{array}{l}\text { 1.5 mi above FS line } \\
0.3 \text { mi below meadow } \\
0.5 \text { mi below FS line } \\
1.0 \text { mi above Green Fly } \\
\text { Below Green Fly Canyon } \\
\text { Upstream of Whitney Ck } \\
\text { Below Coates Ck } \\
3^{\text {rd }} \text { brg above Robinson Fk } \\
2^{\text {nd }} \text { bridge } \\
0.2 \text { mi above Robinson Fk } \\
\text { Holmberg Rd Bridge } \\
\text { Just above mouth }\end{array}$ \\
\hline Coates Creek & $\mathrm{C}-1$ & 0.1 & T8N,R40E,Sect 7,SW1/4,NE1/4 & $\mathrm{T}, \mathrm{F}^{\mathrm{c}}$ & Above Rd \\
\hline Whitney Creek & $\begin{array}{l}\text { WH-1 } \\
\text { WH-2 }\end{array}$ & $\begin{array}{l}3.5 \\
0.3 \\
\end{array}$ & $\begin{array}{l}\text { T8N,R40E,Sect 30,NW1/4,NE } 1 / 4 \\
\text { T8N,R40E,Sect 18,NW1/4,NE1/4 }\end{array}$ & $\begin{array}{l}\mathrm{EL} \\
\mathrm{T}, \mathrm{W}, \mathrm{F}^{\mathrm{c}}\end{array}$ & $\begin{array}{l}\text { Headwaters to FS line } \\
0.2 \text { mi above Wolf Fk Rd. }\end{array}$ \\
\hline Robinson Fork & $\begin{array}{l}\text { RF-1 } \\
\text { RF-2 } \\
\text { RF-3 } \\
\text { RF-4 } \\
\text { RF-5 } \\
\text { RF-6 } \\
\text { RF-7 } \\
\text { RF-8 } \\
\text { RF-9 } \\
\text { RF-10 } \\
\text { RF-11 } \\
\text { RF-12 } \\
\text { RF-13 } \\
\text { RF-14 }\end{array}$ & $\begin{array}{l}7.1 \\
6.6 \\
6.3 \\
6.1 \\
5.6 \\
5.1 \\
4.6 \\
4.5 \\
4.4 \\
4.2 \\
3.8 \\
3.2 \\
2.3 \\
1.5\end{array}$ & 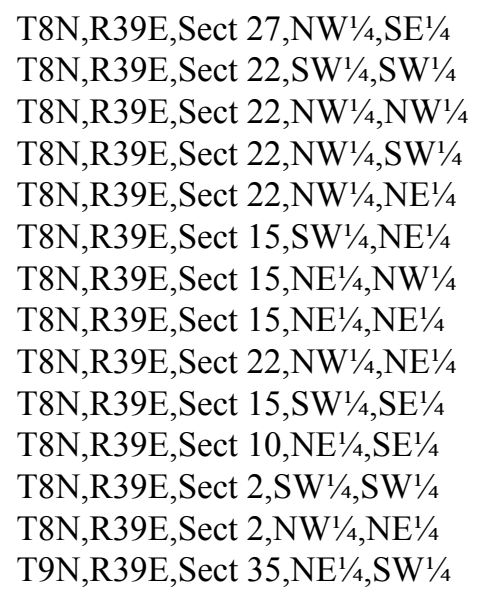 & $\begin{array}{l}\text { EL } \\
\text { EL } \\
\text { EQ,T,W,F }{ }^{c} \\
\text { EL } \\
\text { EL } \\
\text { EL } \\
\text { EL } \\
\text { EL } \\
\text { EL } \\
\text { EL } \\
\text { EQ } \\
\text { EL } \\
\text { EL,EQ } \\
\text { T,W,F }{ }^{c}\end{array}$ & $\begin{array}{l}\text { Robinson Fk tributaries } \\
5.5 \mathrm{mi} \text { above Broughton } \\
6.3 \mathrm{mi} \text { up Robinson Fk Rd } \\
5.0 \mathrm{mi} \text { above Broughton } \\
4.5 \mathrm{mi} \text { above Broughton } \\
4.0 \mathrm{mi} \text { above Broughton } \\
\text { 3.5 mi above Broughton } \\
\text { Up Robinson tributary } \\
\text { 3.0 mi above Broughton } \\
2.5 \mathrm{mi} \text { above Broughton } \\
1.9 \mathrm{mi} \text { above Broughton } \\
2.0 \mathrm{mi} \text { above Broughton } \\
1^{\text {st }} \text { bridge above gate } \\
\text { Below Robinson Fk bridge }\end{array}$ \\
\hline SF Touchet River & $\begin{array}{l}\text { SFT-1 } \\
\text { SFT-2 } \\
\text { SFT-3 } \\
\text { SFT-4 } \\
\text { SFT-5 } \\
\text { SFT-6 } \\
\text { SFT-7 } \\
\text { SFT-8 } \\
\text { SFT-9 }\end{array}$ & $\begin{array}{r}16.6 \\
13.4 \\
9.2 \\
7.2 \\
6.9 \\
5.0 \\
3.0 \\
0.4 \\
0.3\end{array}$ & $\begin{array}{l}\text { T7N,R39E,Sect 8,SW1/4,SW1/4 } \\
\text { T8N,R39E,Sect 31,SE1/4,SE1/4 } \\
\text { T8N,R39E,Sect 8,SE1/4,NW1/4 } \\
\text { T9N,R39E,Sect 32,SE1/4,SE1/4 } \\
\text { T9N,R39E,Sect 32,SE1/4,NE1/4 } \\
\text { T9N,R39E,Sect 28,NW1/4,NE¹/4 } \\
\text { T9N,R39E,Sect 16,NE1/4,NW¹/4 } \\
\text { T10N,R39E,Sect 32,SE1/4,SW1/4 } \\
\text { T10N,R39E,Sect 32,SE1/4,SW1/4 }\end{array}$ & $\begin{array}{l}\mathrm{EQ}^{\mathrm{d}} \\
\mathrm{EQ}^{\mathrm{d}} \\
\mathrm{EQ}^{\mathrm{d}} \\
\mathrm{EQ}^{\mathrm{d}} \\
\mathrm{EQ} \\
\mathrm{EQ}^{\mathrm{d}} \\
\mathrm{EQ}^{\mathrm{d}} \\
\mathrm{S}, \mathrm{EQ}^{\mathrm{d}} \\
\mathrm{T}, \mathrm{F}^{\mathrm{c}}, \mathrm{W}, \mathrm{S}\end{array}$ & $\begin{array}{l}\text { FS line } \\
\text { Below Griffin Fk } \\
\text { Mile post } 9 \\
\text { Mile post } 7 \\
6.5 \text { mi up SF Touchet Rd } \\
\text { Below powerlines } \\
1^{\text {st }} \text { bridge up SFT Rd } \\
0.1 \text { mi above Gephart Rd } \\
\text { Gephart Rd }\end{array}$ \\
\hline $\begin{array}{ll}\text { a } & \text { River mile. } \\
\text { b } & \text { EQ - Quantitat } \\
& \text { Temperature; } \\
\text { c } & \text { Index discharg } \\
\text { d } & \text { Sites electrofis } \\
\end{array}$ & $\begin{array}{l}\text { Flow; } \\
\text { es. } \\
\text { by } \mathrm{Sn}\end{array}$ & Rive & $\begin{array}{l}\text { density estimates); EL - Qualitat } \\
\text { Q Quality; G - Flow gauge. } \\
\text { Lab personnel. }\end{array}$ & trofishin & oorkel; T - \\
\hline
\end{tabular}




\begin{tabular}{|c|c|c|c|c|c|}
\hline Reach & Site \# & $\mathbf{R M}^{\mathbf{a}}$ & Location & $\begin{array}{l}\text { Sample } \\
\text { Type }^{\text {b }}\end{array}$ & Comments \\
\hline SF Patit Creek & $\begin{array}{l}\text { SFP-1 } \\
\text { SFP-2 } \\
\text { SFP-3 }\end{array}$ & $\begin{array}{l}6.7 \\
3.5 \\
1.1\end{array}$ & $\begin{array}{l}\text { T10N,R40E,Sect 25,SE1/4,SE1/4 } \\
\text { T10N,R40E,Sect 22,SE1/4,SW1/4 } \\
\text { T10N,R40E,Sect 22,SE1/4,SW1/4 }\end{array}$ & $\begin{array}{l}\mathrm{T}, \mathrm{W}, \mathrm{F}^{\mathrm{c}} \\
\mathrm{T} \\
\mathrm{EL}\end{array}$ & $\begin{array}{l}\text { End of Road } \\
\text { Blue Gate } \\
1.2 \text { mi up SF Patit Rd }\end{array}$ \\
\hline Touchet River & $\begin{array}{l}\text { TR-1 } \\
\text { TR-2 } \\
\text { TR-3 } \\
\text { TR-4 } \\
\text { TR-5 } \\
\text { TR-6 } \\
\text { TR-7 } \\
\text { TR-8 } \\
\text { TR-9 } \\
\text { TR-10 }\end{array}$ & $\begin{array}{r}54.5 \\
54.0 \\
53.8 \\
53.5 \\
52.3 \\
48.4 \\
40.5 \\
27.4 \\
11.3 \\
1.5\end{array}$ & 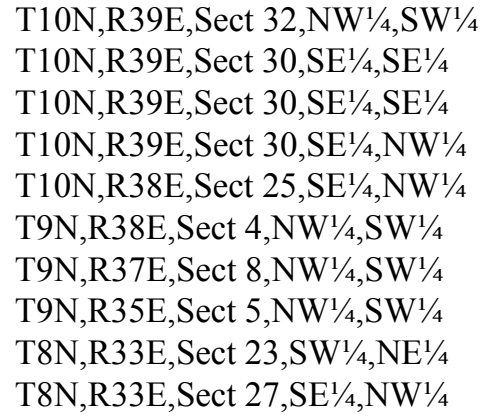 & $\begin{array}{l}\text { EQ,S } \\
\mathrm{S} \\
\mathrm{T}, \mathrm{S} \\
\mathrm{EQ}, \mathrm{F}^{\mathrm{c}} \\
\mathrm{EQ} \\
\mathrm{S}, \mathrm{T} \\
\mathrm{T}, \mathrm{F}^{\mathrm{c}} \\
\mathrm{T} \\
\mathrm{T}, \mathrm{F}^{\mathrm{c}}, \mathrm{G}, \mathrm{W} \\
\mathrm{T}, \mathrm{F}^{\mathrm{c}}, \mathrm{G}\end{array}$ & $\begin{array}{l}\text { N \& S fork confluence } \\
\text { Above intake } \\
\text { Snake River Lab } \\
\text { Flag pole } \\
\text { Sewer plant } \\
\text { L \& C State Park } \\
\text { Bolles Br. } \\
\text { Lamar Rd } \\
\text { Below Simms Rd. Br. } \\
\text { Cummins Bridge }\end{array}$ \\
\hline South Fork Coppei & $\begin{array}{l}\text { SFC-1 } \\
\text { SFC-2 } \\
\text { SFC-3 } \\
\text { SFC-4 } \\
\text { SFC-5 }\end{array}$ & $\begin{array}{l}4.8 \\
3.5 \\
3.2 \\
1.1 \\
0.8\end{array}$ & $\begin{array}{l}\text { T8N,R38E,Sect 33,NW1/4,NE1/4 } \\
\text { T8N,R38E,Sect 22,NE1/4,NE1/4 } \\
\text { T8N,R38E,Sect 20,SE1/4,SE1/4 } \\
\text { T8N,R38E,Sect 18,NW1/4,NE1/4 } \\
\text { T8N,R38E,Sect 18,NW1/4,NE1/4 }\end{array}$ & $\begin{array}{l}\text { EQ } \\
\text { EL } \\
T, F^{c} \\
\text { EL } \\
\text { EQ }\end{array}$ & $\begin{array}{l}\text { Below Barnes Rd./Ck } \\
\text { Geir Road } \\
\text { Canyon Culvert } \\
1 \text { mi above Walker Rd Brg } \\
\text { Walker Rd Bridge }\end{array}$ \\
\hline North Fork Coppei & $\begin{array}{l}\text { NFC-1 } \\
\text { NFC-2 } \\
\text { NFC-3 } \\
\text { NFC-4 } \\
\text { NFC-5 }\end{array}$ & $\begin{array}{l}5.2 \\
3.3 \\
1.1 \\
0.8 \\
0.1\end{array}$ & $\begin{array}{l}\text { T8N,R38E,Sect 27,SE1/4,NE1/4 } \\
\text { T8N,R38E,Sect 21,NE1/4,NW1/4 } \\
\text { T8N,R38E,Sect 8,SW1/4,SE1/4 } \\
\text { T8N,R38E,Sect 8,SW1/4,NW1/4 } \\
\text { T8N,R38E,Sect 7,NW } 1 / 4, \mathrm{NW}^{1 / 4}\end{array}$ & $\begin{array}{l}\text { EQ } \\
\text { EL } \\
\text { EQ } \\
\text { EL,T,F }{ }^{c} \\
\text { EQ }\end{array}$ & $\begin{array}{l}\text { DNR gate } \\
2.1 \text { mi below DNR gate } \\
\text { Power lines } \\
\text { Grain Elevators } \\
\text { Forks Bridge }\end{array}$ \\
\hline Mainstem Coppei & $\begin{array}{l}\text { MC-1 } \\
\text { MC-2 } \\
\text { MC-3 } \\
\text { MC-4 }\end{array}$ & $\begin{array}{l}4.0 \\
5.8 \\
4.6 \\
1.8\end{array}$ & $\begin{array}{l}\text { T9N,R37E,Sect 24,NW } 1 / 4, \mathrm{SW}^{1 / 4} \\
\text { T9N,R37E,Sect 36,SE1/4,SW1/4 } \\
\text { T9N,R37E,Sect 25,SW1/4,SE1/4 } \\
\text { T9N,R37E,Sect 14,NW } 1 / 4, \mathrm{SE}^{1 / 4}\end{array}$ & $\begin{array}{l}\text { EL } \\
\text { EL } \\
\text { EQ,T,F }{ }^{c} \\
\text { EQ,EL }\end{array}$ & $\begin{array}{l}0.5 \text { mi below McCowen Brg } \\
\text { Below power lines } \\
\text { Above McCowen } \mathrm{Rd} \text {. Br } \\
\text { Below Meinberg Rd. Br. }\end{array}$ \\
\hline Dry Creek & $\begin{array}{l}\text { DC-1 } \\
\text { DC-2 } \\
\text { DC-3 } \\
\text { DC-4 } \\
\text { DC-5 } \\
\text { DC-6 }\end{array}$ & $\begin{array}{r}31.2 \\
29.4 \\
28.8 \\
27.3 \\
17.4 \\
3.4 \\
\end{array}$ & 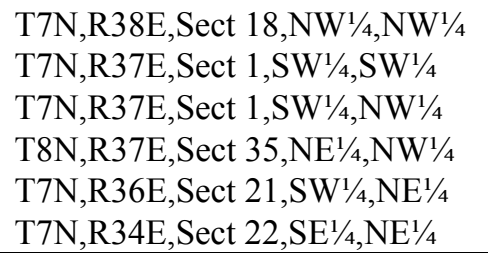 & $\begin{array}{l}\text { EQ } \\
\text { EQ } \\
\text { EQ } \\
\text { EQ,T,F } F^{c}, W \\
\text { T } \\
\text { T }\end{array}$ & $\begin{array}{l}\text { Biscuit Ridge bridge } \\
1.5 \text { mi below Biscuit Rd Br } \\
2.1 \text { mi up Biscuit Ridge Rd } \\
0.5 \text { mi up Biscuit Ridge Rd } \\
\text { Low Waitsburg Rd. Bridge } \\
\text { Talbott Rd Bridge }\end{array}$ \\
\hline Burnt Fork & $\begin{array}{l}\text { BF-1 } \\
\text { BF-2 } \\
\text { BF-3 }\end{array}$ & $\begin{array}{l}2.8 \\
2.4 \\
2.3\end{array}$ & $\begin{array}{l}\text { T7N,R39E,Sect 16,NE1/4,SW1/4 } \\
\text { T7N,R39E,Sect 16,NW1/4,NE1/4 } \\
\text { T7N,R39E,Sect 16,NW1/4,NW1/4 }\end{array}$ & $\begin{array}{l}\text { EL } \\
\text { EL } \\
\text { EQ }\end{array}$ & $\begin{array}{l}\text { Just below trib fork } \\
0.4 \text { mi below trib fork } \\
\text { Just above FS line }\end{array}$ \\
\hline N Fork Dry Crk & $\begin{array}{l}\text { NFD-1 } \\
\text { NFD-2 } \\
\text { NFD-3 } \\
\text { NFD-4 } \\
\text { NFD-5 } \\
\text { NFD-6 }\end{array}$ & $\begin{array}{l}4.8 \\
1.7 \\
1.1 \\
0.8 \\
0.4 \\
0.0\end{array}$ & 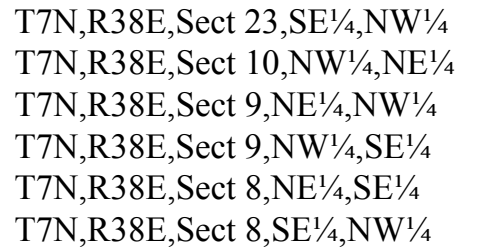 & $\begin{array}{l}\text { EL } \\
\text { EQ } \\
\text { EQ } \\
\text { EQ } \\
\text { T, F } \\
\text { EQ }\end{array}$ & $\begin{array}{l}\text { End of road } \\
4^{\text {th }} \text { stream crossing } \\
3^{\text {rd }} \text { stream crossing } \\
0.8 \text { mi up Scott Rd } \\
0.4 \text { mi up Scott Rd } \\
\text { NFD \& SFD confluence }\end{array}$ \\
\hline \multicolumn{6}{|c|}{$\begin{array}{ll}\text { a } & \text { River mile. } \\
\text { b } & \text { EQ - Quantitative Electrofishing (density estimates); } \\
\text { Temperature; F - Flow; W - Water Quality; G - Flov } \\
\text { c Index discharge sites. } \\
\text { d Sites electrofished by Snake River Lab personnel. }\end{array}$} \\
\hline
\end{tabular}




\begin{tabular}{|c|c|c|c|c|c|}
\hline Reach & Site \# & $\mathbf{R M}^{\mathbf{a}}$ & Location & $\begin{array}{l}\text { Sample } \\
\text { Type }^{\text {b }}\end{array}$ & Comments \\
\hline Mill Ck & $\begin{array}{l}\text { MC-1 } \\
\text { MC-2 } \\
\text { MC-3 } \\
\text { MC-4 } \\
\text { MC-5 } \\
\text { MC-6 } \\
\text { MC-7 } \\
\text { MC-8 } \\
\text { MC-9 } \\
\text { MC-10 } \\
\text { MC-11 } \\
\text { MC-12 } \\
\text { MC-13 } \\
\text { MC-14 } \\
\text { MC-15 } \\
\text { MC-16 } \\
\text { MC-17 } \\
\text { MC-18 } \\
\text { MC-19 } \\
\text { MC-20 } \\
\text { MC-21 }\end{array}$ & $\begin{array}{r}11.5 \\
11.4 \\
10.6 \\
10.5 \\
10.4 \\
10.4 \\
10.3 \\
10.1 \\
8.9 \\
8.1 \\
6.7 \\
6.7 \\
6.6 \\
4.8 \\
4.7 \\
4.1 \\
3.7 \\
3.3 \\
2.7 \\
1.2 \\
0.4\end{array}$ & 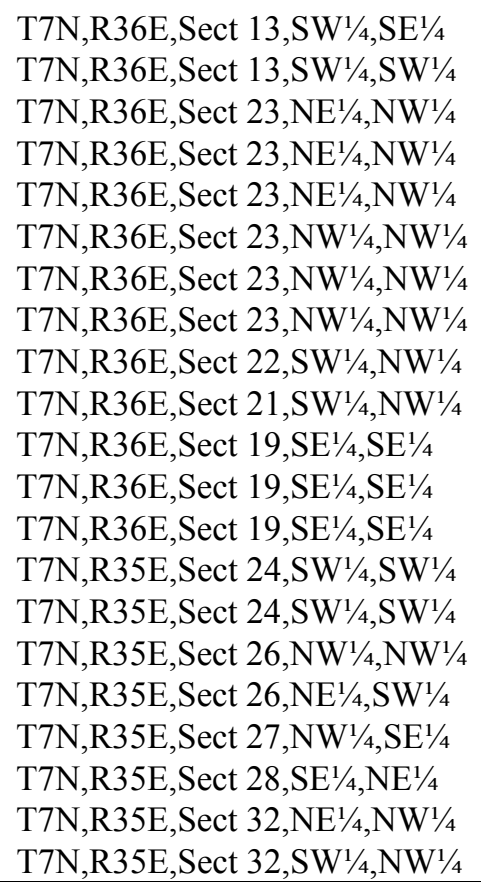 & $\begin{array}{l}\text { EL } \\
\text { EL } \\
\text { EL } \\
\text { EL } \\
\text { EL } \\
\text { EL } \\
\text { EL } \\
\text { EL } \\
\text { EL } \\
\text { F } \\
\text { EL } \\
\text { EL } \\
\text { F } \\
\text { EL } \\
\text { EL } \\
\text { EL,EQ } \\
\text { EL } \\
\text { EL } \\
\text { EL } \\
\text { EQ } \\
\text { EQ,F }\end{array}$ & $\begin{array}{l}\text { Rooks Park } \\
\text { Below Rooks Park } \\
\text { two weirs above diversion } \\
\text { Above diversion } \\
\text { At diversion } \\
\text { Below diversion } \\
\text { Across from WWCC } \\
\text { Below Titus Ck mouth } \\
\text { Above Wilbur Rd bridge } \\
\text { Wildwood Park } \\
9^{\text {th }} \text { street bridge } \\
9^{\text {th }} \text { street to Roosevelt } \\
\text { Below } 9^{\text {th }} \text { street bridge } \\
\text { Above Gose St bridge } \\
\text { Below Gose St bridge } \\
2.2 \text { mi above Wallula Br } \\
1.4 \text { mi above Wallula Br } \\
0.9 \text { mi above Wallula Br } \\
\text { Wallula Rd bridge } \\
0.2 \text { mi below Last Chance Rd } \\
\text { Swegle Rd }\end{array}$ \\
\hline Titus Ck & $\begin{array}{l}\text { TC-1 } \\
\text { TC-2 } \\
\text { TC-3 }\end{array}$ & $\begin{array}{l}0.3 \\
0.2 \\
0.1\end{array}$ & $\begin{array}{l}\text { T7N,R36E,Sect 14,SE 1/4,SW1/4 } \\
\text { T7N,R36E,Sect 23,NW1/4,NE1/4 } \\
\text { T7N,R36E,Sect 23,NW1/4,NE } 1 / 4\end{array}$ & $\begin{array}{l}\text { EL,F } \\
\text { EL } \\
\text { EL }\end{array}$ & $\begin{array}{l}\text { Above WWCC boundary } \\
\text { WWCC nursing building } \\
\text { Footbridge at WWCC }\end{array}$ \\
\hline Walla Walla $\mathrm{R}$ & $\begin{array}{l}\text { WW-1 } \\
\text { WW-2 } \\
\text { WW-3 } \\
\text { WW-4 } \\
\text { WW-5 } \\
\text { WW-6 } \\
\text { WW-7 } \\
\text { WW-8 } \\
\text { WW-9 } \\
\text { WW-10 } \\
\text { WW-11 }\end{array}$ & $\begin{array}{l}39.7 \\
39.6 \\
38.1 \\
37.2 \\
36.7 \\
36.5 \\
35.4 \\
34.0 \\
32.9 \\
29.3 \\
22.7 \\
\end{array}$ & 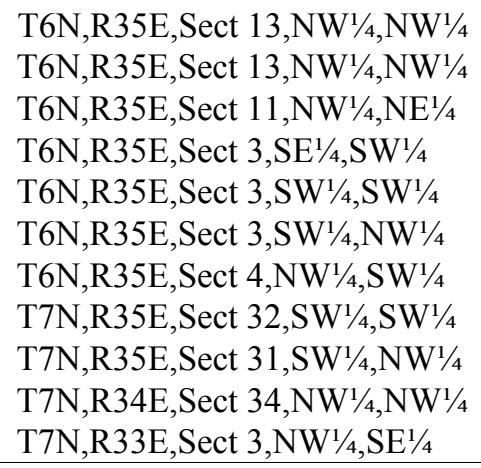 & $\begin{array}{l}\text { EQ,S } \\
\text { W,T,F } F^{c}, G \\
\text { EQ,EL } \\
\text { EQ,F' } \\
\text { S } \\
\text { EQ,F', W,G,T } \\
\text { W } \\
\text { EQ,T,F } \\
\text { S,T,F } F^{c}, W, G \\
\text { S,T,F } \\
\text { T }\end{array}$ & $\begin{array}{l}\text { Below Birch Ck mouth } \\
\text { Pepper Bridge Rd Br } \\
\text { Above Yellowhawk mouth } \\
0.5 \text { mi above Burlingame } \\
\text { Burlingame Diversion } \\
\text { Below Mojonnier Rd. } \\
\text { Above Last Chance Rd Br } \\
\text { Below Swegle Rd Br } \\
\text { Above Detour Rd Br } \\
\text { Above McDonald Rd Br } \\
\text { Touchet-Gardena Rd }\end{array}$ \\
\hline Birch Ck & $\mathrm{BC}-1$ & 0.1 & T6N,R35E,Sect13,NW1/4,NW1/4 & $\mathrm{F}$ & At mouth Walla Walla R. \\
\hline W. Little Walla & $\begin{array}{l}\text { WLW-1 } \\
\text { WLW-2 } \\
\text { WLW-3 } \\
\text { WLW-4 }\end{array}$ & $\begin{array}{r}4.1 \\
3 \\
0.7 \\
0.6\end{array}$ & $\begin{array}{l}\text { T6N,R35E,Sect 9,SE1/4,NW1/4 } \\
\text { T6N,R35E,Sect 5,SE1/4,SE1/4 } \\
\text { T6N,R35E,Sect 5,NW1/4,NW1/4 } \\
\text { T7N,R35E,Sect 31,SE¹/4,SE1/4 }\end{array}$ & $\begin{array}{l}\text { EL, } F^{c} \\
\text { EL,F } \\
\text { EL } \\
\text { EL }\end{array}$ & $\begin{array}{l}0.6 \text { mi up Valley Chapel Rd } \\
\text { Frog Hollow Rd } \\
\text { East of Swegle Rd } \\
\text { West of Swegle Rd }\end{array}$ \\
\hline E. Little Walla & $\begin{array}{l}\text { ELW-1 } \\
\text { ELW-2 } \\
\text { ELW-3 } \\
\text { ELW-4 }\end{array}$ & $\begin{array}{l}1.5 \\
1.0 \\
0.6 \\
0.2\end{array}$ & $\begin{array}{l}\text { T6N,R35E,Sect 14,SE1/4,SE1/4 } \\
\text { T6N,R35E,Sect 11,NW1/4,NW1/4 } \\
\text { T6N,R35E,Sect 10,SE1/4,SE1/4 } \\
\text { T6N,R35E,Sect 10,NW } 1 / 4, \mathrm{NW}^{1 / 4}\end{array}$ & $\begin{array}{l}\mathrm{EQ}, \mathrm{F}^{\mathrm{c}} \\
\mathrm{EQ} \\
\mathrm{EQ} \\
\mathrm{EL}\end{array}$ & $\begin{array}{l}\text { At river fork } \\
0.5 \text { mi below river fork } \\
\text { Springdale Rd Bridge } \\
\text { Upstream of mouth }\end{array}$ \\
\hline \multicolumn{6}{|c|}{$\begin{array}{l}\text { a River mile. } \\
\text { bQ - Quantitative Electrofishing (density estimates); EL - Qualitative electrofishing; S - Snorkel; T - } \\
\text { Temperature; F - Flow; W - Water Quality; G - Flow gauge. } \\
\text { c Index discharge sites. }\end{array}$} \\
\hline
\end{tabular}




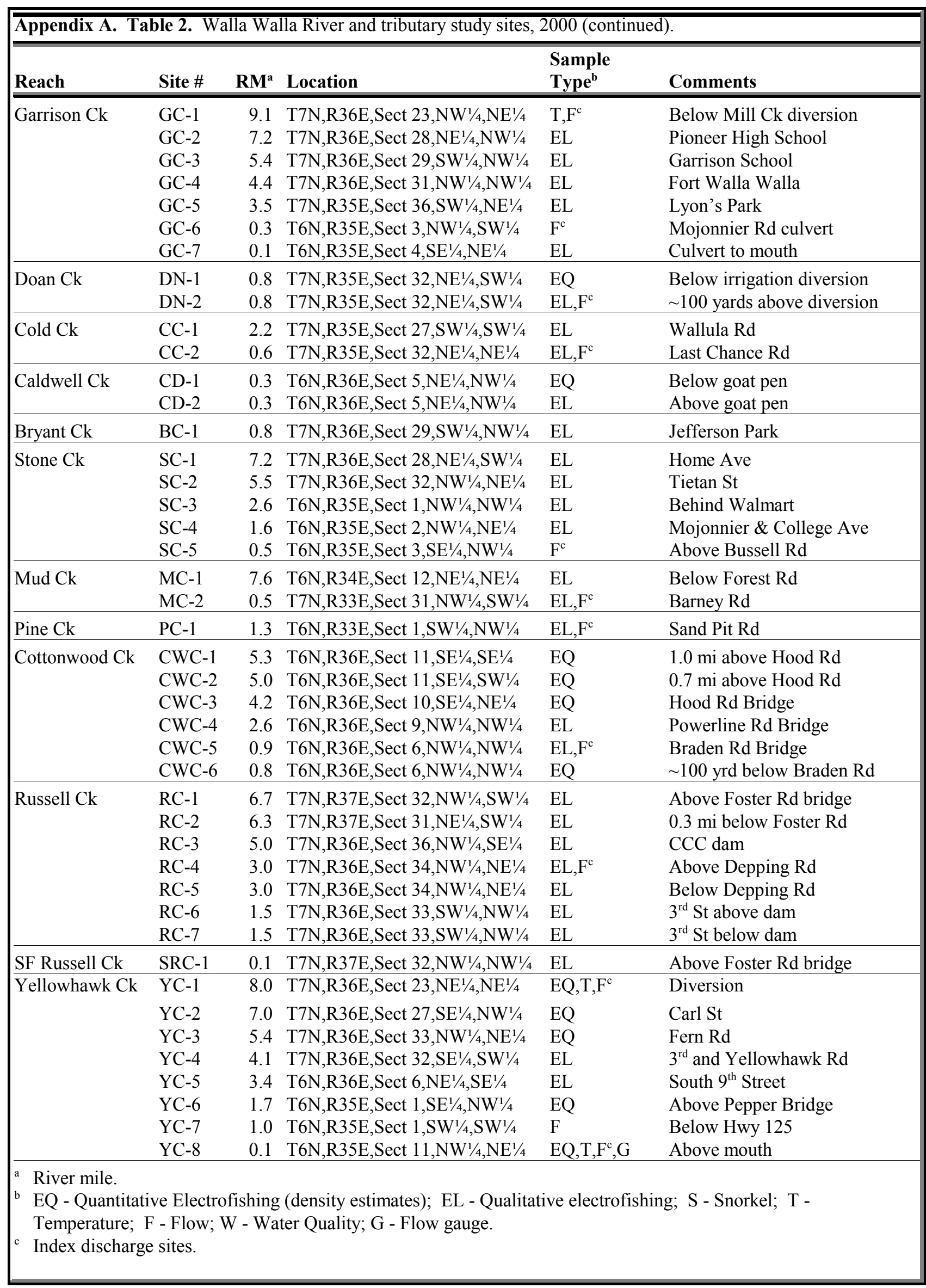




\section{Appendix B. Discharge Data 2000}

Assessment of Salmonids and Their Habitat Conditions in 


\begin{tabular}{|c|c|c|c|c|c|c|}
\hline Stream & Site & Date & CFS & $\operatorname{Temp}(\mathbf{F})$ & Time & Comments \\
\hline NF Touchet R & NFT-7 & $\begin{array}{r}5 / 4 \\
5 / 17 \\
6 / 1 \\
6 / 12 \\
6 / 27 \\
7 / 10 \\
7 / 20 \\
8 / 3 \\
8 / 21 \\
9 / 11 \\
9 / 19 \\
10 / 5 \\
10 / 18 \\
11 / 1 \\
11 / 15 \\
\end{array}$ & $\begin{array}{r}60.9 \\
44.2 \\
33.1 \\
47.5 \\
19.0 \\
11.3 \\
10.0 \\
7.7 \\
6.5 \\
8.4 \\
6.4 \\
6.0 \\
6.1 \\
6.6 \\
5.6 \\
\end{array}$ & $\begin{array}{l}40 \\
40 \\
42 \\
44 \\
50 \\
54 \\
51 \\
56 \\
50 \\
48 \\
57 \\
40 \\
45 \\
38 \\
33 \\
\end{array}$ & $\begin{array}{r}10: 27 \\
11: 29 \\
9: 48 \\
11: 46 \\
11: 15 \\
14: 10 \\
08: 48 \\
12: 29 \\
10: 55 \\
10: 15 \\
11: 47 \\
09: 13 \\
10: 47 \\
09: 31 \\
10: 42 \\
\end{array}$ & 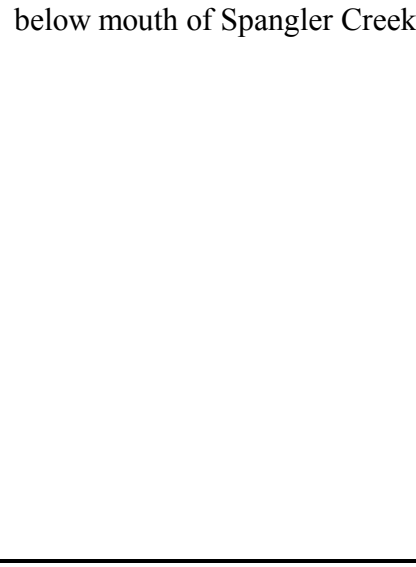 \\
\hline NF Touchet R & NFT-11 & $\begin{array}{r}5 / 4 \\
5 / 17 \\
6 / 1 \\
6 / 13 \\
6 / 27 \\
7 / 11 \\
7 / 20 \\
8 / 3 \\
8 / 21 \\
9 / 11 \\
9 / 19 \\
10 / 5 \\
10 / 18 \\
11 / 1 \\
11 / 15\end{array}$ & $\begin{array}{l}89.4 \\
72.0 \\
58.1 \\
59.2 \\
38.2 \\
33.7 \\
29.5 \\
21.9 \\
23.2 \\
31.0 \\
21.6 \\
24.8 \\
23.7 \\
26.2 \\
26.9\end{array}$ & $\begin{array}{r}47 \\
47 \\
51 \\
51 \\
60 \\
53 \\
58 \\
65 \\
58 \\
54.5 \\
59 \\
45.5 \\
49 \\
44 \\
35\end{array}$ & $\begin{array}{l}11: 50 \\
12: 05 \\
10: 56 \\
13: 21 \\
12: 23 \\
08: 25 \\
09: 42 \\
13: 20 \\
12: 00 \\
11: 45 \\
13: 02 \\
09: 58 \\
11: 28 \\
10: 21 \\
11: 28\end{array}$ & 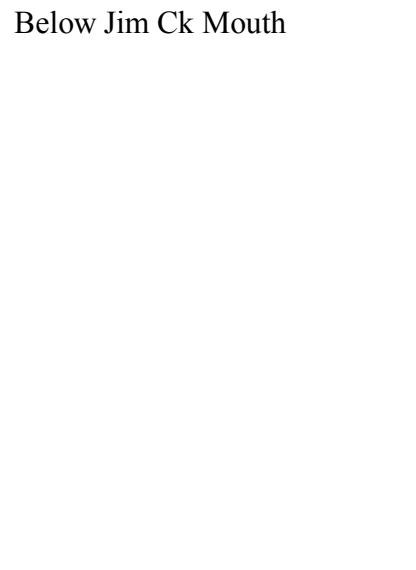 \\
\hline NF Touchet R & NFT-16 & $\begin{array}{r}5 / 25 \\
6 / 6 \\
6 / 15 \\
6 / 29 \\
7 / 11 \\
7 / 20 \\
8 / 9 \\
8 / 21 \\
9 / 8 \\
9 / 19 \\
10 / 5 \\
10 / 18 \\
11 / 1 \\
11 / 17 \\
\end{array}$ & \begin{tabular}{|r|}
114.5 \\
182.9 \\
131.6 \\
69.2 \\
63.9 \\
52.3 \\
47.3 \\
45.2 \\
43.2 \\
43.3 \\
52.6 \\
48.1 \\
53.6 \\
52.2 \\
\end{tabular} & $\begin{array}{l}61 \\
62 \\
55 \\
58 \\
61 \\
65 \\
61 \\
67 \\
58 \\
65 \\
52 \\
53 \\
47 \\
39 \\
\end{array}$ & $\begin{array}{l}15: 00 \\
13: 44 \\
10: 35 \\
09: 23 \\
10: 40 \\
11: 38 \\
09: 45 \\
14: 40 \\
15: 00 \\
15: 06 \\
12: 53 \\
13: 21 \\
12: 24 \\
14: 44 \\
\end{array}$ & Above Baileysburg \\
\hline Corral Ck & $\mathrm{CC}-3$ & $7 / 31$ & 0.5 & 52 & $13: 35$ & Snow park \\
\hline
\end{tabular}




\begin{tabular}{|c|c|c|c|c|c|c|}
\hline Stream & Site & Date & CFS & $\operatorname{Temp}(\mathbf{F})$ & Time & Comments \\
\hline Spangler Creek & SC-6 & $\begin{array}{r}5 / 4 \\
5 / 17 \\
6 / 1 \\
6 / 12 \\
6 / 27 \\
7 / 10 \\
7 / 20 \\
8 / 3 \\
8 / 21 \\
9 / 11 \\
9 / 19 \\
10 / 5 \\
10 / 18 \\
11 / 1 \\
11 / 15 \\
\end{array}$ & $\begin{array}{r}18.1 \\
13.3 \\
9.8 \\
13.2 \\
4.9 \\
4.3 \\
3.7 \\
3.1 \\
2.7 \\
3.1 \\
2.2 \\
2.1 \\
2.2 \\
2.3 \\
1.9 \\
\end{array}$ & $\begin{array}{r}41 \\
40 \\
40 \\
45 \\
49 \\
52 \\
50 \\
55 \\
48 \\
47 \\
52 \\
39 \\
44 \\
37.5 \\
32 \\
\end{array}$ & $\begin{array}{r}09: 45 \\
11: 09 \\
9: 13 \\
12: 03 \\
10: 40 \\
13: 55 \\
08: 30 \\
12: 16 \\
10: 35 \\
09: 45 \\
11: 33 \\
08: 58 \\
10: 36 \\
09: 16 \\
10: 30 \\
\end{array}$ & . \\
\hline Lewis Creek & LC-12 & $\begin{array}{r}5 / 4 \\
5 / 17 \\
6 / 1 \\
6 / 12 \\
6 / 27 \\
7 / 10 \\
7 / 20 \\
8 / 3 \\
8 / 21 \\
9 / 11 \\
9 / 19 \\
10 / 5 \\
10 / 18 \\
11 / 1 \\
11 / 15 \\
\end{array}$ & $\begin{array}{r}12.5 \\
8.7 \\
9.0 \\
8.7 \\
6.6 \\
7.0 \\
6.4 \\
6.0 \\
4.8 \\
5.6 \\
4.8 \\
4.8 \\
4.8 \\
4.8 \\
5.3 \\
\end{array}$ & $\begin{array}{r}44 \\
48 \\
46 \\
48 \\
53 \\
56 \\
52 \\
53 \\
49 \\
49.5 \\
53.5 \\
43 \\
46 \\
42 \\
37 \\
\end{array}$ & $\begin{array}{l}10: 55 \\
14: 14 \\
10: 12 \\
12: 37 \\
11: 34 \\
14: 27 \\
09: 03 \\
12: 48 \\
11: 15 \\
10: 50 \\
12: 50 \\
09: 31 \\
11: 01 \\
09: 45 \\
11: 06 \\
\end{array}$ & 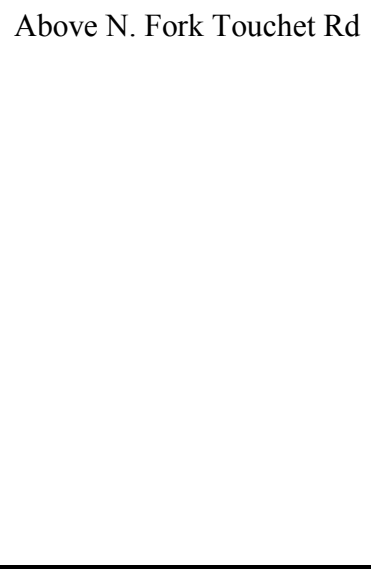 \\
\hline Jim Creek & JC-1 & $\begin{array}{r}5 / 4 \\
5 / 17 \\
6 / 1 \\
6 / 13 \\
6 / 27 \\
7 / 11 \\
7 / 20 \\
8 / 3 \\
8 / 21 \\
9 / 11 \\
9 / 19 \\
10 / 5 \\
10 / 18 \\
11 / 1 \\
11 / 15 \\
\end{array}$ & $\begin{array}{l}2.8 \\
2.7 \\
2.8 \\
2.9 \\
1.7 \\
1.1 \\
1.0 \\
1.0 \\
1.2 \\
1.2 \\
1.0 \\
1.1 \\
1.1 \\
1.3 \\
1.3 \\
\end{array}$ & $\begin{array}{r}49 \\
55 \\
51 \\
53 \\
60 \\
61 \\
58 \\
66 \\
55 \\
54.5 \\
59 \\
43 \\
49 \\
40 \\
35 \\
\end{array}$ & $\begin{array}{l}11: 21 \\
14: 38 \\
10: 33 \\
13: 00 \\
12: 00 \\
14: 50 \\
09: 27 \\
13: 04 \\
11: 35 \\
11: 25 \\
12: 50 \\
09: 45 \\
11: 13 \\
10: 03 \\
11: 28 \\
\end{array}$ & Culvert at Jim Ck Rd \\
\hline Wolf Fork & WF-5 & $\begin{array}{r}5 / 17 \\
6 / 19 \\
9 / 8 \\
10 / 11 \\
11 / 15\end{array}$ & $\begin{array}{l}41.6 \\
25.1 \\
16.9 \\
16.5 \\
14.4\end{array}$ & $\begin{array}{l}41 \\
48 \\
45 \\
44 \\
37\end{array}$ & $\begin{array}{l}13: 45 \\
12: 59 \\
12: 25 \\
12: 24 \\
12: 37\end{array}$ & Below Green Fly Canyon \\
\hline
\end{tabular}




\begin{tabular}{|c|c|c|c|c|c|c|}
\hline Stream & Site & Date & CFS & $\operatorname{Temp}(\mathbf{F})$ & Time & Comments \\
\hline Wolf Fork & WF-9 & $\begin{array}{r}6 / 1 \\
6 / 12 \\
6 / 27 \\
7 / 11 \\
7 / 20 \\
8 / 3 \\
8 / 21 \\
9 / 8 \\
9 / 19 \\
10 / 5 \\
10 / 18 \\
11 / 1 \\
11 / 15 \\
\end{array}$ & $\begin{array}{l}65.3 \\
77.0 \\
35.3 \\
33.2 \\
32.1 \\
28.2 \\
26.5 \\
27.8 \\
24.7 \\
26.9 \\
27.6 \\
28.8 \\
28.0 \\
\end{array}$ & $\begin{array}{r}54 \\
48 \\
58.5 \\
50 \\
54 \\
52 \\
56 \\
51 \\
56 \\
45 \\
48 \\
44 \\
37 \\
\end{array}$ & $\begin{array}{c}12: 54 \\
14: 51 \\
13: 42 \\
09: 40 \\
10: 44 \\
14: 20 \\
13: 20 \\
15: 13 \\
14: 14 \\
11: 42 \\
12: 28 \\
11: 19 \\
13: 55 \\
\end{array}$ & 更 \\
\hline Wolf Fork & WF-11 & $\begin{array}{r}5 / 4 \\
5 / 17 \\
6 / 1 \\
6 / 12 \\
6 / 27 \\
7 / 11 \\
7 / 20 \\
8 / 9 \\
8 / 21 \\
9 / 8 \\
9 / 19 \\
10 / 5 \\
10 / 18 \\
11 / 1 \\
11 / 15 \\
\end{array}$ & \begin{tabular}{|r|}
122.5 \\
76.2 \\
89.9 \\
93.4 \\
32.4 \\
28.1 \\
27.0 \\
26.1 \\
20.5 \\
20.7 \\
21.1 \\
21.5 \\
24.1 \\
23.5 \\
25.0 \\
\end{tabular} & $\begin{array}{l}50 \\
50 \\
57 \\
51 \\
65 \\
55 \\
59 \\
55 \\
60 \\
55 \\
59 \\
48 \\
49 \\
44 \\
37 \\
\end{array}$ & $\begin{array}{l}14: 13 \\
10: 48 \\
13: 55 \\
15: 45 \\
14: 51 \\
10: 20 \\
11: 17 \\
10: 16 \\
14: 13 \\
14: 49 \\
14: 47 \\
12: 27 \\
13: 00 \\
12: 06 \\
14: 25 \\
\end{array}$ & . \\
\hline Coates Creek & $\mathrm{C}-1$ & $\begin{array}{r}5 / 4 \\
5 / 17 \\
6 / 1 \\
6 / 14 \\
6 / 27 \\
7 / 11 \\
7 / 20 \\
8 / 3 \\
8 / 21 \\
9 / 8 \\
9 / 19 \\
10 / 5 \\
10 / 18 \\
11 / 1 \\
11 / 15 \\
\end{array}$ & $\begin{array}{l}7.5 \\
5.9 \\
6.0 \\
8.9 \\
3.1 \\
2.5 \\
2.4 \\
3.2 \\
1.9 \\
2.0 \\
2.0 \\
2.3 \\
1.9 \\
2.4 \\
2.2 \\
\end{array}$ & $\begin{array}{r}45 \\
46 \\
49 \\
55 \\
54.5 \\
51 \\
55 \\
58 \\
53 \\
50 \\
55.5 \\
43 \\
49 \\
41 \\
37 \\
\end{array}$ & $\begin{array}{l}13: 13 \\
14: 45 \\
12: 30 \\
14: 31 \\
13: 26 \\
09: 27 \\
10: 26 \\
13: 55 \\
12: 51 \\
14: 50 \\
13: 55 \\
11: 18 \\
12: 11 \\
11: 00 \\
13: 33 \\
\end{array}$ & . \\
\hline Whitney Creek & WC-2 & $\begin{array}{r}5 / 4 \\
5 / 17 \\
6 / 1 \\
6 / 12 \\
6 / 27 \\
7 / 11 \\
7 / 20 \\
8 / 3 \\
8 / 21\end{array}$ & $\begin{array}{r}13.4 \\
10.0 \\
8.7 \\
14.9 \\
6.7 \\
5.2 \\
4.3 \\
3.2 \\
4.1\end{array}$ & $\begin{array}{r}45 \\
45 \\
48 \\
47 \\
54.5 \\
51 \\
54 \\
57 \\
57\end{array}$ & $\begin{array}{l}12: 46 \\
14: 21 \\
12: 09 \\
14: 11 \\
13: 00 \\
09: 03 \\
10: 13 \\
13: 50 \\
12: 35\end{array}$ & 0.2 mi up Whitney Creek \\
\hline
\end{tabular}




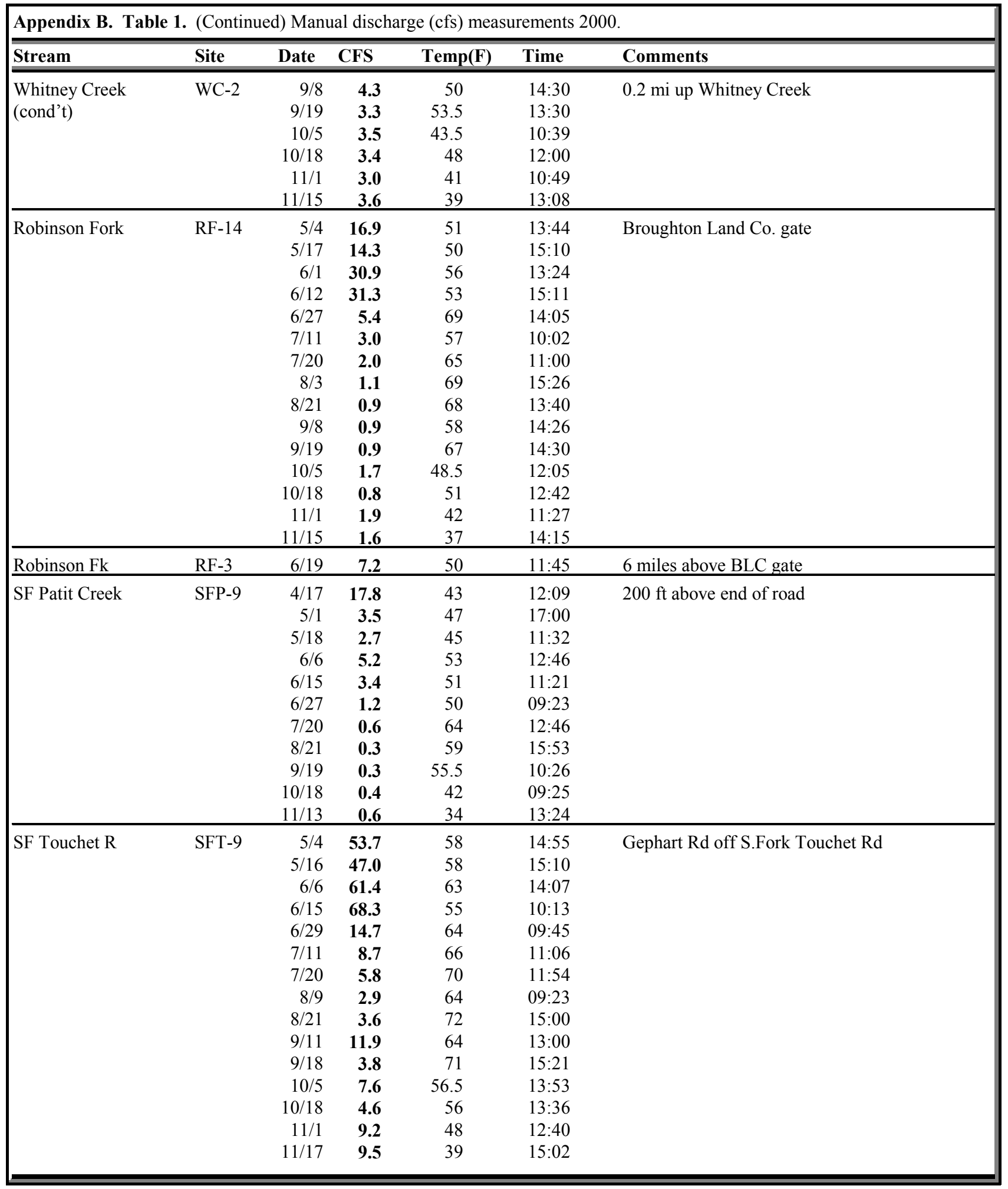




\begin{tabular}{|c|c|c|c|c|c|c|}
\hline Stream & Site & Date & CFS & Temp(F) & Time & Comments \\
\hline Touchet River & TR-4 & $\begin{array}{r}4 / 17 \\
5 / 12 \\
5 / 18 \\
6 / 6 \\
6 / 14 \\
6 / 29 \\
7 / 12 \\
7 / 20 \\
8 / 8 \\
8 / 21 \\
9 / 7 \\
9 / 18 \\
10 / 5 \\
10 / 23 \\
11 / 6 \\
11 / 20 \\
\end{array}$ & $\begin{array}{r}377.2 \\
204.9 \\
192.4 \\
187.7 \\
211.5 \\
78.5 \\
62.5 \\
57.4 \\
39.4 \\
43.0 \\
45.0 \\
48.7 \\
62.6 \\
67.6 \\
65.5 \\
61.6 \\
\end{array}$ & $\begin{array}{r}52 \\
47 \\
54 \\
63 \\
66 \\
62 \\
63 \\
71 \\
72 \\
67 \\
68 \\
65.5 \\
53 \\
44 \\
45 \\
34 \\
\end{array}$ & $\begin{array}{l}13: 25 \\
09: 08 \\
12: 30 \\
14: 50 \\
15: 15 \\
10: 20 \\
11: 25 \\
13: 40 \\
17: 00 \\
16: 45 \\
15: 20 \\
02: 56 \\
14: 24 \\
09: 44 \\
12: 35 \\
09: 15 \\
\end{array}$ & 更 \\
\hline Touchet River & TR-7 & $\begin{array}{r}4 / 11 \\
5 / 1 \\
5 / 16 \\
6 / 6 \\
6 / 29 \\
7 / 12 \\
8 / 8 \\
8 / 22 \\
9 / 5 \\
9 / 18 \\
10 / 4 \\
10 / 23 \\
11 / 1 \\
11 / 17 \\
\end{array}$ & $\begin{array}{r}297.3 \\
220.7 \\
211.8 \\
265.2 \\
82.1 \\
64.5 \\
36.9 \\
32.8 \\
49.2 \\
47.9 \\
68.4 \\
72.8 \\
72.1 \\
76.7 \\
\end{array}$ & $\begin{array}{r}57.5 \\
58 \\
60 \\
57 \\
69 \\
66 \\
77 \\
59 \\
57 \\
62.5 \\
49 \\
45 \\
50 \\
38 \\
\end{array}$ & $\begin{array}{r}15: 30 \\
15: 59 \\
13: 10 \\
08: 40 \\
10: 55 \\
10: 47 \\
16: 07 \\
8: 43 \\
09: 00 \\
09: 08 \\
08: 46 \\
09: 11 \\
14: 33 \\
13: 35 \\
\end{array}$ & (1) \\
\hline Touchet River & TR-9 & $\begin{array}{r}4 / 11 \\
5 / 1 \\
5 / 15 \\
5 / 25 \\
6 / 6 \\
6 / 20 \\
6 / 29 \\
7 / 10 \\
7 / 20 \\
8 / 7 \\
8 / 21 \\
9 / 5 \\
9 / 18 \\
10 / 4 \\
10 / 19 \\
11 / 2 \\
11 / 16\end{array}$ & $\begin{array}{r}303.2 \\
203.3 \\
199.0 \\
177.4 \\
243.5 \\
141.7 \\
69.9 \\
63.9 \\
40.0 \\
20.0 \\
27.5 \\
47.2 \\
39.4 \\
72.6 \\
50.6 \\
63.5 \\
78.6\end{array}$ & $\begin{array}{r}60 \\
63 \\
69 \\
64 \\
64 \\
68 \\
75 \\
75 \\
78 \\
70 \\
62 \\
60 \\
66.5 \\
50 \\
51 \\
41 \\
33.8\end{array}$ & $\begin{array}{l}14: 38 \\
14: 53 \\
15: 28 \\
13: 10 \\
09: 50 \\
12: 35 \\
11: 50 \\
13: 25 \\
13: 20 \\
13: 05 \\
10: 30 \\
10: 14 \\
10: 12 \\
09: 53 \\
07: 50 \\
09: 05 \\
09: 45\end{array}$ & Below Simms Road Br. \\
\hline
\end{tabular}




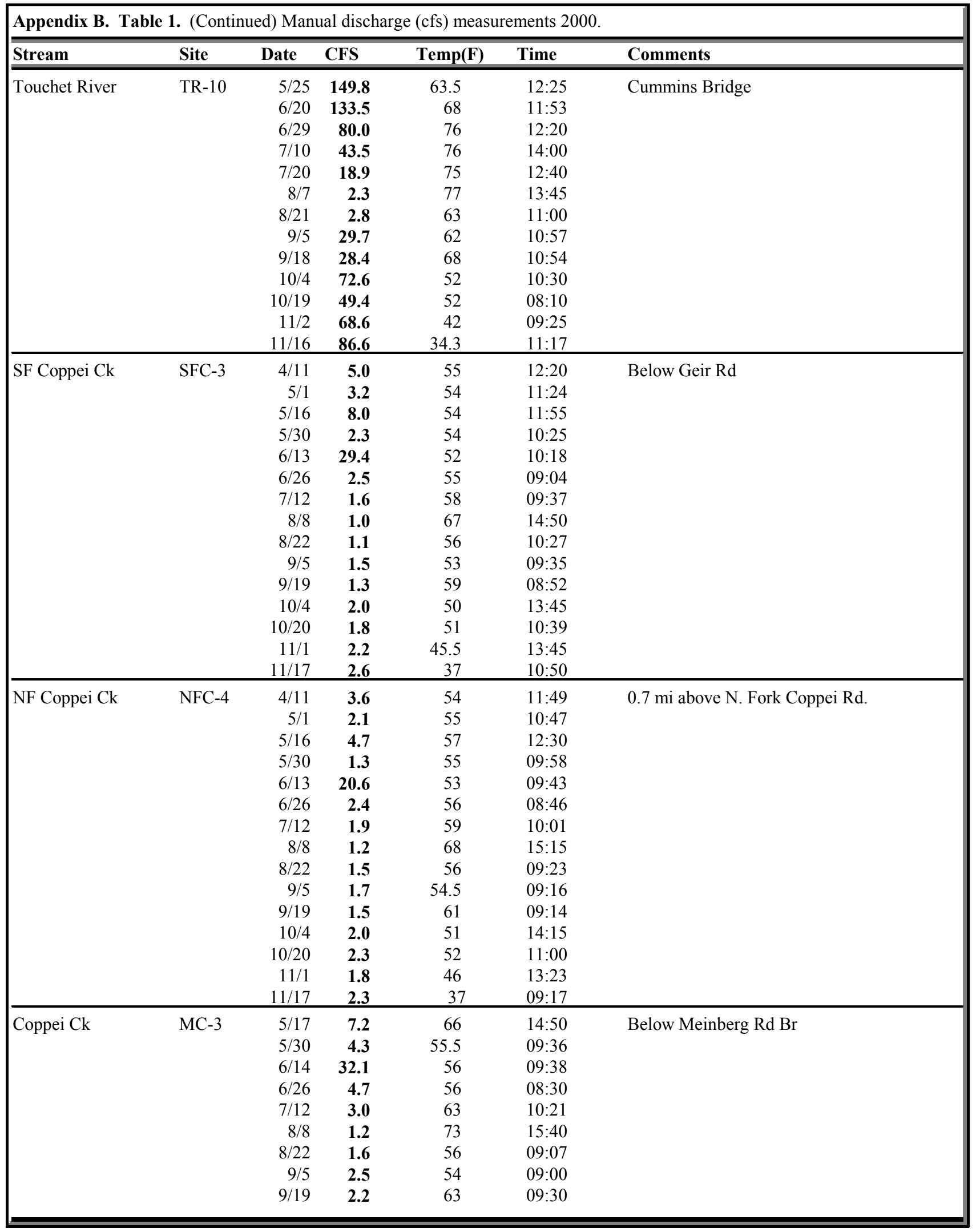




\begin{tabular}{|c|c|c|c|c|c|c|}
\hline Stream & Site & Date & CFS & Temp(F) & Time & Comments \\
\hline Coppei Ck (Cont'd) & $\mathrm{MC}-3$ & $\begin{array}{r}10 / 4 \\
10 / 20 \\
11 / 1 \\
11 / 17 \\
\end{array}$ & $\begin{array}{l}4.3 \\
3.7 \\
4.6 \\
5.9 \\
\end{array}$ & $\begin{array}{r}54.5 \\
53 \\
49 \\
36 \\
\end{array}$ & $\begin{array}{l}14: 36 \\
11: 30 \\
14: 05 \\
08: 45 \\
\end{array}$ & Below Meinberg Road Br. \\
\hline Dry Creek & DC-4 & $\begin{array}{r}4 / 11 \\
4 / 24 \\
5 / 1 \\
5 / 16 \\
5 / 30 \\
6 / 13 \\
6 / 26 \\
7 / 12 \\
8 / 8 \\
8 / 22 \\
9 / 5 \\
9 / 18 \\
10 / 4 \\
10 / 19 \\
11 / 6 \\
11 / 17 \\
\end{array}$ & $\begin{array}{r}16.5 \\
14.3 \\
9.1 \\
14.0 \\
4.4 \\
52.6 \\
5.5 \\
2.7 \\
1.0 \\
1.2 \\
1.9 \\
1.3 \\
4.1 \\
2.4 \\
3.7 \\
4.6 \\
\end{array}$ & $\begin{array}{r}53 \\
53 \\
57 \\
64 \\
55 \\
53 \\
57 \\
60 \\
72 \\
57.5 \\
55 \\
64 \\
51 \\
53 \\
43 \\
36.5 \\
\end{array}$ & $\begin{array}{l}12: 41 \\
14: 50 \\
12: 30 \\
14: 26 \\
11: 24 \\
11: 37 \\
09: 57 \\
09: 12 \\
14: 20 \\
11: 20 \\
10: 22 \\
15: 45 \\
13: 15 \\
14: 25 \\
11: 45 \\
11: 10\end{array}$ & 更 \\
\hline NF Dry Ck & NFD-5 & $\begin{array}{r}5 / 16 \\
5 / 30 \\
6 / 13 \\
6 / 26 \\
7 / 12 \\
8 / 8 \\
8 / 22 \\
9 / 5 \\
9 / 18 \\
10 / 4 \\
10 / 19 \\
11 / 6 \\
11 / 17 \\
\end{array}$ & $\begin{array}{r}7.9 \\
1.8 \\
19.2 \\
2.2 \\
1.4 \\
1.1 \\
0.7 \\
0.9 \\
1.1 \\
1.4 \\
1.0 \\
2.1 \\
2.1 \\
\end{array}$ & $\begin{array}{r}60 \\
52 \\
50.5 \\
53 \\
55 \\
64 \\
54 \\
53.5 \\
59 \\
48 \\
50 \\
42 \\
39 \\
\end{array}$ & $\begin{array}{l}14: 00 \\
11: 00 \\
11: 03 \\
09: 34 \\
08: 49 \\
14: 00 \\
10: 56 \\
10: 04 \\
15: 23 \\
12: 53 \\
14: 10 \\
11: 05 \\
10: 40 \\
\end{array}$ & 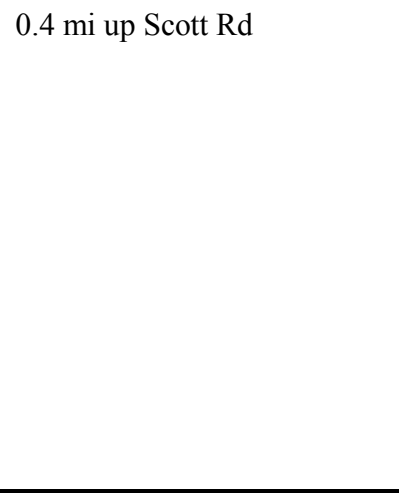 \\
\hline Walla Walla R. & WW-2 & $\begin{array}{r}5 / 16 \\
5 / 26 \\
5 / 30 \\
6 / 20 \\
6 / 29 \\
7 / 11 \\
7 / 20 \\
8 / 7 \\
8 / 21 \\
9 / 5 \\
9 / 18 \\
9 / 26 \\
9 / 18 \\
9 / 26 \\
10 / 4 \\
10 / 19 \\
11 / 2 \\
11 / 19\end{array}$ & $\begin{array}{r}226.7 \\
91.7 \\
57.9 \\
79.3 \\
5.5 \\
3.6 \\
4.0 \\
4.1 \\
* 0.2 \\
* 0.3 \\
4.3 \\
5.3 \\
4.7 \\
5.7 \\
81.0 \\
23.7 \\
48.6 \\
107.6\end{array}$ & $\begin{array}{r}58 \\
51.5 \\
56 \\
56 \\
71 \\
72 \\
65 \\
66 \\
69 \\
61 \\
63.5 \\
52 \\
63.5 \\
52 \\
50.5 \\
53.5 \\
47 \\
38\end{array}$ & $\begin{array}{r}12: 40 \\
9: 50 \\
13: 14 \\
9: 20 \\
14: 30 \\
15: 06 \\
09: 30 \\
10: 15 \\
16: 15 \\
11: 50 \\
12: 30 \\
09: 50 \\
12: 00 \\
09: 02 \\
11: 10 \\
12: 35 \\
13: 20 \\
13: 52\end{array}$ & $\begin{array}{l}\text { Above Pepper Bridge } \\
\text { *equipment failure } \\
\text { *equipment failure }\end{array}$ \\
\hline
\end{tabular}




\begin{tabular}{|c|c|c|c|c|c|c|}
\hline Stream & Site & Date & CFS & $\operatorname{Temp}(\mathbf{F})$ & Time & Comments \\
\hline Walla Walla R. & WW-4 & $\begin{array}{r}5 / 30 \\
6 / 26 \\
7 / 11 \\
8 / 7 \\
8 / 8 \\
9 / 5 \\
9 / 18 \\
10 / 19 \\
11 / 2 \\
11 / 16 \\
\end{array}$ & $\begin{array}{r}113.6 \\
83.0 \\
45.1 \\
27.6 \\
33.1 \\
48.7 \\
48.1 \\
81.9 \\
119.3 \\
193.2 \\
\end{array}$ & $\begin{array}{r}56.5 \\
63 \\
71 \\
65 \\
66 \\
57 \\
61.5 \\
52 \\
46 \\
38 \\
\end{array}$ & $\begin{array}{l}13: 59 \\
11: 50 \\
14: 15 \\
11: 20 \\
14: 23 \\
13: 17 \\
10: 50 \\
11: 20 \\
12: 15 \\
15: 24\end{array}$ & Above Burlingame Diversion \\
\hline Walla Walla R. & WW-6 & $\begin{array}{r}5 / 1 \\
5 / 15 \\
5 / 25 \\
5 / 30 \\
6 / 20 \\
6 / 29 \\
7 / 11 \\
7 / 20 \\
8 / 7 \\
8 / 21 \\
9 / 5 \\
9 / 18 \\
10 / 4 \\
10 / 19 \\
11 / 2 \\
11 / 16 \\
\end{array}$ & $\begin{array}{r}187.2 \\
260.1 \\
64.9 \\
30.0 \\
104.6 \\
10.5 \\
16.4 \\
42.2 \\
29.9 \\
32.2 \\
49.0 \\
47.7 \\
93.4 \\
16.5 \\
16.9 \\
118.5 \\
\end{array}$ & $\begin{array}{r}56 \\
61 \\
57 \\
57 \\
59 \\
71 \\
71 \\
69 \\
\mathrm{~N} / \mathrm{A} \\
65 \\
59.5 \\
61.5 \\
49 \\
52 \\
46 \\
41 \\
\end{array}$ & $\begin{array}{l}14: 00 \\
14: 10 \\
15: 28 \\
14: 23 \\
10: 21 \\
13: 40 \\
13: 54 \\
10: 45 \\
16: 45 \\
14: 05 \\
13: 40 \\
10: 30 \\
09: 28 \\
11: 00 \\
12: 00 \\
14: 53 \\
\end{array}$ & 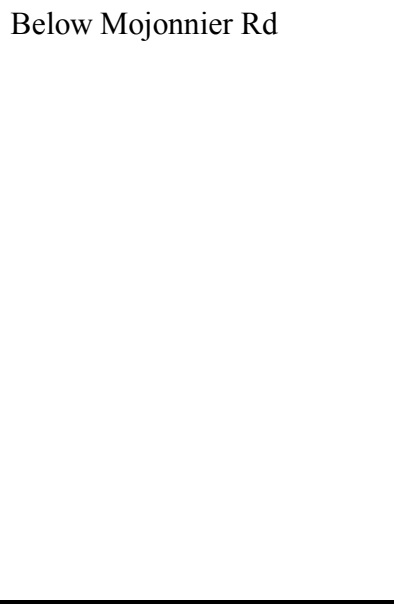 \\
\hline Walla Walla R. & WW-8 & $\begin{array}{r}5 / 16 \\
6 / 26 \\
7 / 11 \\
8 / 7 \\
8 / 21 \\
9 / 5 \\
9 / 18 \\
10 / 4 \\
10 / 19 \\
11 / 2 \\
11 / 16 \\
11 / 17 \\
\end{array}$ & $\begin{array}{r}220.0 \\
42.9 \\
22.2 \\
29.1 \\
36.7 \\
54.8 \\
56.3 \\
97.1 \\
23.7 \\
24.4 \\
122.6 \\
122.0 \\
\end{array}$ & $\begin{array}{r}58 \\
68 \\
73 \\
74 \\
64 \\
59 \\
65.5 \\
52.5 \\
52 \\
47 \\
40.5 \\
38.3 \\
\end{array}$ & $\begin{array}{l}12: 15 \\
14: 16 \\
12: 43 \\
16: 15 \\
12: 35 \\
13: 05 \\
13: 14 \\
12: 03 \\
09: 35 \\
11: 20 \\
14: 06 \\
11: 54\end{array}$ & 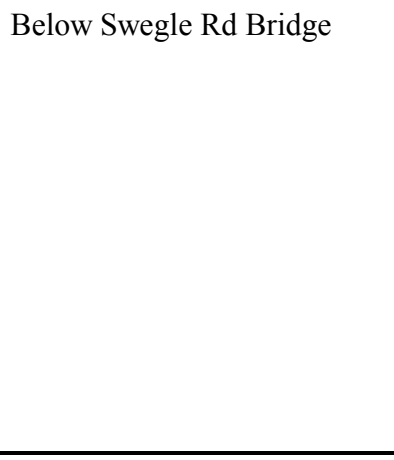 \\
\hline Walla Walla R. & WW-9 & $\begin{array}{r}4 / 11 \\
5 / 1 \\
5 / 15 \\
5 / 25 \\
6 / 20 \\
6 / 29 \\
7 / 10 \\
7 / 20 \\
8 / 7 \\
8 / 21 \\
9 / 5 \\
9 / 18 \\
10 / 4\end{array}$ & $\begin{array}{r}428.1 \\
258.4 \\
360.1 \\
124.2 \\
171.7 \\
22.7 \\
25.6 \\
38.5 \\
29.3 \\
38.1 \\
55.0 \\
59.9 \\
128.2\end{array}$ & $\begin{array}{r}52 \\
57 \\
62 \\
59 \\
62 \\
76 \\
75 \\
74 \\
75 \\
65 \\
60 \\
65.5 \\
53\end{array}$ & $\begin{array}{l}13: 40 \\
13: 25 \\
14: 41 \\
14: 30 \\
10: 58 \\
13: 05 \\
16: 00 \\
12: 00 \\
15: 30 \\
12: 17 \\
12: 28 \\
12: 40 \\
12: 32\end{array}$ & Below Detour Rd Bridge \\
\hline
\end{tabular}




\begin{tabular}{|c|c|c|c|c|c|c|}
\hline Stream & Site & Date & CFS & $\operatorname{Temp}(\mathbf{F})$ & Time & Comments \\
\hline $\begin{array}{l}\text { Walla Walla R. } \\
\text { (Cont'd) }\end{array}$ & WW-9 & $\begin{array}{r}10 / 19 \\
11 / 2 \\
11 / 16 \\
\end{array}$ & $\begin{array}{r}41.7 \\
44.7 \\
152.5 \\
\end{array}$ & $\begin{array}{l}52 \\
47 \\
41 \\
\end{array}$ & $\begin{array}{l}09: 17 \\
10: 30 \\
13: 20 \\
\end{array}$ & Below Detour Rd Bridge \\
\hline Walla Walla R. & WW-10 & $\begin{array}{r}6 / 26 \\
7 / 10 \\
8 / 7 \\
8 / 21 \\
9 / 5 \\
9 / 18 \\
10 / 4 \\
10 / 19 \\
11 / 2 \\
11 / 16 \\
\end{array}$ & $\begin{array}{r}36.6 \\
5.9 \\
11.0 \\
17.8 \\
34.3 \\
41.0 \\
112.2 \\
27.3 \\
35.2 \\
136.3 \\
\end{array}$ & $\begin{array}{r}76 \\
81 \\
81 \\
69 \\
61 \\
67.5 \\
54 \\
52 \\
46.5 \\
38 \\
\end{array}$ & $\begin{array}{l}14: 45 \\
14: 45 \\
15: 05 \\
11: 55 \\
11: 54 \\
12: 04 \\
12: 00 \\
08: 55 \\
10: 00 \\
10: 39 \\
\end{array}$ & Above McDonald Rd Bridge \\
\hline Birch Ck & & $\begin{array}{r}9 / 5 \\
9 / 18 \\
\end{array}$ & $\begin{array}{l}0.2 \\
0.3 \\
\end{array}$ & $\begin{array}{l}63 \\
63 \\
\end{array}$ & $\begin{array}{l}12: 01 \\
12: 40 \\
\end{array}$ & State line \\
\hline Yellowhawk Ck & YC-1 & $\begin{array}{l}5 / 25 \\
5 / 30 \\
\end{array}$ & $\begin{array}{r}38.5 \\
40.9 \\
\end{array}$ & $\begin{array}{l}59 \\
55 \\
\end{array}$ & $\begin{array}{l}12: 01 \\
11: 58 \\
\end{array}$ & Above diversion \\
\hline Yellowhawk Ck & YC-1 & $\begin{array}{r}6 / 13 \\
6 / 26 \\
7 / 11 \\
8 / 8 \\
8 / 22 \\
9 / 5 \\
9 / 18 \\
10 / 4 \\
10 / 19 \\
11 / 2 \\
11 / 17 \\
\end{array}$ & $\begin{array}{l}46.3 \\
35.0 \\
24.1 \\
15.8 \\
14.5 \\
28.9 \\
26.3 \\
20.7 \\
34.1 \\
48.4 \\
42.3 \\
\end{array}$ & $\begin{array}{c}54 \\
61 \\
75 \\
73 \\
65 \\
55 \\
66 \\
\text { NA } \\
54 \\
45 \\
39 \\
\end{array}$ & $\begin{array}{r}12: 30 \\
10: 31 \\
16: 31 \\
13: 15 \\
11: 54 \\
10: 58 \\
14: 22 \\
\text { NA } \\
13: 38 \\
14: 47 \\
12: 04 \\
\end{array}$ & Below diversion \\
\hline Yellowhawk Ck & YC-7 & $\begin{array}{l}5 / 17 \\
5 / 30 \\
6 / 13 \\
\end{array}$ & $\begin{array}{l}50.6 \\
43.3 \\
94.1 \\
\end{array}$ & $\begin{array}{l}58 \\
56 \\
56 \\
\end{array}$ & $\begin{array}{l}12: 20 \\
13: 32 \\
13: 52 \\
\end{array}$ & Highway 125 \\
\hline Yellowhawk Ck & YC-8 & $\begin{array}{r}5 / 26 \\
6 / 20 \\
6 / 29 \\
7 / 11 \\
7 / 20 \\
8 / 7 \\
8 / 21 \\
9 / 5 \\
9 / 18 \\
10 / 4 \\
10 / 19 \\
11 / 2 \\
11 / 16 \\
\end{array}$ & $\begin{array}{l}42.8 \\
50.0 \\
41.3 \\
28.6 \\
27.0 \\
18.5 \\
18.3 \\
26.3 \\
30.4 \\
30.8 \\
41.5 \\
50.0 \\
50.7 \\
\end{array}$ & $\begin{array}{r}56 \\
58 \\
68 \\
68 \\
75 \\
68 \\
63 \\
57 \\
62 \\
49 \\
54 \\
44 \\
38.8 \\
\end{array}$ & $\begin{array}{l}10: 50 \\
09: 48 \\
14: 02 \\
14: 32 \\
10: 00 \\
10: 40 \\
15: 18 \\
12: 31 \\
11: 07 \\
10: 00 \\
11: 32 \\
12: 30 \\
15: 50 \\
\end{array}$ & Above mouth \\
\hline Yellowhawk Ck & $\mathrm{YC}-2$ & $5 / 17$ & 24.6 & 54 & $10: 27$ & Carl St. \\
\hline Mill Creek & $\mathrm{MC}-13$ & $8 / 10$ & 3.0 & 71.5 & $15: 47$ & $9^{\text {th }}$ Street \\
\hline Mill Creek & MC-10 & $8 / 10$ & 0.4 & 57 & $17: 15$ & Wildwood Park \\
\hline Titus Creek & $\mathrm{TC}-1$ & $9 / 18$ & 2.7 & 64 & $14: 40$ & Community College \\
\hline
\end{tabular}




\begin{tabular}{|c|c|c|c|c|c|c|}
\hline Stream & Site & Date & CFS & $\operatorname{Temp}(\mathbf{F})$ & Time & Comments \\
\hline Mill Creek & MC-20 & $\begin{array}{r}5 / 17 \\
6 / 6 \\
6 / 26 \\
7 / 10 \\
8 / 7 \\
8 / 21 \\
9 / 5 \\
9 / 18 \\
10 / 4 \\
10 / 19 \\
11 / 2 \\
11 / 18 \\
\end{array}$ & $\begin{array}{r}71.8 \\
130.6 \\
10.3 \\
2.8 \\
1.1 \\
2.2 \\
3.7 \\
6.1 \\
37.6 \\
11.3 \\
13.1 \\
21.9 \\
\end{array}$ & $\begin{array}{r}65 \\
57 \\
70 \\
76 \\
76 \\
68 \\
64 \\
67.5 \\
56 \\
54 \\
49 \\
41.5 \\
\end{array}$ & $\begin{array}{l}14: 05 \\
10: 34 \\
13: 39 \\
16: 15 \\
16: 00 \\
13: 14 \\
13: 25 \\
13: 43 \\
13: 50 \\
10: 10 \\
10: 45 \\
11: 07\end{array}$ & Below Swegle Rd \\
\hline Garrison $\mathrm{Ck}$ & GC-1 & $\begin{array}{r}6 / 13 \\
6 / 26 \\
7 / 11 \\
8 / 8 \\
8 / 22 \\
9 / 5 \\
9 / 18 \\
10 / 4 \\
10 / 19 \\
11 / 2 \\
11 / 17 \\
\end{array}$ & $\begin{array}{l}5.6 \\
4.1 \\
4.0 \\
3.4 \\
4.8 \\
6.0 \\
3.8 \\
2.4 \\
5.0 \\
4.2 \\
4.3 \\
\end{array}$ & $\begin{array}{c}55 \\
61 \\
75 \\
73 \\
64 \\
54 \\
66 \\
\text { NA } \\
\text { NA } \\
45 \\
39 \\
\end{array}$ & $\begin{array}{r}12: 08 \\
10: 20 \\
16: 20 \\
13: 03 \\
11: 45 \\
10: 46 \\
14: 10 \\
\text { NA } \\
\text { NA } \\
14: 40 \\
11: 45 \\
\end{array}$ & 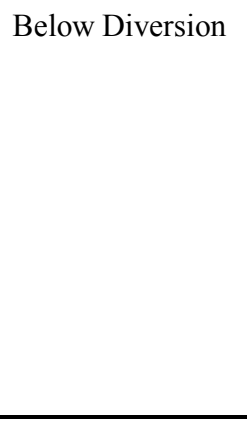 \\
\hline Garrison $\mathrm{Ck}$ & GC-6 & $\begin{array}{r}5 / 17 \\
5 / 30 \\
6 / 14 \\
6 / 26 \\
7 / 11 \\
8 / 8 \\
8 / 21 \\
9 / 5 \\
9 / 18 \\
10 / 4 \\
10 / 19 \\
11 / 2 \\
11 / 18 \\
\end{array}$ & $\begin{array}{r}4.9 \\
5.3 \\
8.8 \\
3.5 \\
2.6 \\
-- \\
1.9 \\
2.2 \\
3.1 \\
5.2 \\
3.6 \\
4.2 \\
5.4 \\
\end{array}$ & $\begin{array}{r}64 \\
60 \\
62 \\
65 \\
69 \\
\text { NA } \\
65 \\
61 \\
61 \\
53 \\
54 \\
47 \\
40.5 \\
\end{array}$ & $\begin{array}{l}13: 38 \\
14: 40 \\
11: 23 \\
12: 20 \\
13: 36 \\
00: 00 \\
13: 48 \\
14: 04 \\
10: 25 \\
09: 08 \\
10: 37 \\
11: 45 \\
12: 35 \\
\end{array}$ & $\begin{array}{l}\text { Mission Rd } \\
\text { No flow }\end{array}$ \\
\hline $\begin{array}{l}\text { E. Little Walla } \\
\text { Walla }\end{array}$ & ELW-1 & $\begin{array}{r}4 / 25 \\
5 / 15 \\
6 / 2 \\
6 / 13 \\
6 / 26 \\
7 / 11 \\
8 / 7 \\
8 / 21 \\
9 / 5 \\
9 / 18 \\
10 / 4 \\
10 / 19 \\
11 / 2 \\
11 / 18\end{array}$ & $\begin{array}{r}9.1 \\
9.3 \\
10.0 \\
11.3 \\
9.4 \\
6.0 \\
6.2 \\
5.5 \\
9.6 \\
11.9 \\
12.4 \\
10.7 \\
10.7 \\
8.9\end{array}$ & $\begin{array}{r}50 \\
58 \\
54 \\
61 \\
57 \\
66 \\
58 \\
61 \\
55 \\
56.5 \\
48 \\
50.5 \\
48.5 \\
44.5\end{array}$ & $\begin{array}{r}13: 38 \\
11: 12 \\
9: 31 \\
14: 15 \\
11: 30 \\
14: 50 \\
11: 05 \\
15: 00 \\
12: 46 \\
11: 25 \\
10: 18 \\
11: 50 \\
12: 50 \\
13: 03\end{array}$ & Above river fork \\
\hline
\end{tabular}




\begin{tabular}{|c|c|c|c|c|c|c|}
\hline Stream & Site & Date & CFS & $\operatorname{Temp}(\mathbf{F})$ & Time & Comments \\
\hline Stone Creek & SC-5 & $\begin{array}{r}5 / 26 \\
6 / 2 \\
6 / 13 \\
6 / 26 \\
7 / 11 \\
11 / 18 \\
\end{array}$ & \begin{tabular}{r|}
0.2 \\
1.8 \\
1.6 \\
0.3 \\
-- \\
0.5 \\
\end{tabular} & $\begin{array}{r}67 \\
63.5 \\
64 \\
70 \\
\\
43.5 \\
\end{array}$ & $\begin{array}{r}11: 51 \\
9: 48 \\
14: 38 \\
12: 06 \\
12: 47 \\
\end{array}$ & $\begin{array}{l}\text { Above Bussell Rd } \\
\text { No water }\end{array}$ \\
\hline Russell Ck & $\mathrm{RC}-4$ & $\begin{array}{l}5 / 17 \\
5 / 30 \\
6 / 13 \\
6 / 26 \\
7 / 11 \\
7 / 20 \\
\end{array}$ & $\begin{array}{r}2.8 \\
1.2 \\
13.7 \\
1.9 \\
0.4 \\
--\end{array}$ & $\begin{array}{l}58 \\
56 \\
55 \\
59 \\
67\end{array}$ & $\begin{array}{l}11: 32 \\
12: 22 \\
12: 57 \\
10: 53 \\
15: 49\end{array}$ & $\begin{array}{l}\text { Above Depping Rd } \\
\text { No measurable flow }\end{array}$ \\
\hline Reser Ck & & $5 / 17$ & 0.3 & 63 & $11: 07$ & Reser Creek Rd \\
\hline Cottonwood Ck & CWC-5 & $\begin{array}{r}4 / 25 \\
5 / 15 \\
5 / 30 \\
6 / 13 \\
6 / 26 \\
7 / 11 \\
8 / 8 \\
8 / 22 \\
9 / 5 \\
10 / 4 \\
11 / 2 \\
11 / 17 \\
\end{array}$ & $\begin{array}{r}9.7 \\
13.1 \\
1.3 \\
30.8 \\
1.6 \\
1.2 \\
0.4 \\
0.2 \\
0.1 \\
2.2 \\
0.5 \\
2.4 \\
\end{array}$ & $\begin{array}{r}50 \\
70 \\
55.5 \\
64 \\
55 \\
60 \\
58 \\
57 \\
54 \\
49 \\
51 \\
42 \\
\end{array}$ & $\begin{array}{l}11: 25 \\
13: 21 \\
12: 49 \\
13: 23 \\
11: 10 \\
15: 32 \\
12: 20 \\
12: 21 \\
11: 26 \\
11: 25 \\
13: 35 \\
12: 41 \\
\end{array}$ & Braden Rd \\
\hline Doan Creek & DN-2 & $\begin{array}{r}5 / 25 \\
6 / 2 \\
6 / 14 \\
6 / 26 \\
7 / 11 \\
8 / 8 \\
8 / 21 \\
9 / 5 \\
9 / 18 \\
10 / 4 \\
10 / 19 \\
11 / 2 \\
11 / 17\end{array}$ & $\begin{array}{l}1.4 \\
2.1 \\
2.6 \\
1.9 \\
1.7 \\
0.6 \\
0.5 \\
0.7 \\
0.8 \\
0.5 \\
1.2 \\
1.5 \\
1.3\end{array}$ & $\begin{array}{r}59 \\
59 \\
61 \\
60 \\
63 \\
61 \\
62 \\
58.5 \\
60 \\
54 \\
53 \\
47 \\
43.2 \\
\end{array}$ & $\begin{array}{l}11: 24 \\
10: 58 \\
12: 50 \\
13: 57 \\
12: 15 \\
10: 30 \\
12: 52 \\
14: 09 \\
09: 43 \\
13: 28 \\
09: 55 \\
11: 06 \\
11: 28\end{array}$ & Whitman Mission \\
\hline Cold Creek & $\mathrm{CC}-2$ & $\begin{array}{r}5 / 26 \\
6 / 2 \\
6 / 14 \\
6 / 26 \\
7 / 11 \\
8 / 21 \\
9 / 5 \\
9 / 18 \\
10 / 4 \\
10 / 19 \\
11 / 2 \\
11 / 18\end{array}$ & $\begin{array}{l}1.2 \\
2.6 \\
1.0 \\
0.7 \\
0.7 \\
0.2 \\
0.6 \\
1.2 \\
2.2 \\
1.9 \\
1.8 \\
1.7\end{array}$ & $\begin{array}{r}61 \\
61 \\
63.5 \\
62 \\
62 \\
57 \\
56 \\
61.5 \\
52 \\
53 \\
44.5 \\
38.3\end{array}$ & $\begin{array}{l}12: 20 \\
10: 35 \\
12: 25 \\
13: 20 \\
13: 03 \\
13: 33 \\
14: 23 \\
10: 00 \\
14: 15 \\
10: 25 \\
11: 35 \\
12: 17\end{array}$ & Swegle Rd \\
\hline
\end{tabular}




\begin{tabular}{|c|c|c|c|c|c|c|}
\hline Stream & Site & Date & CFS & $\operatorname{Temp}(\mathbf{F})$ & Time & Comments \\
\hline $\begin{array}{l}\text { West Little Walla } \\
\text { Walla }\end{array}$ & WLW-1 & $\begin{array}{r}6 / 14 \\
6 / 26 \\
7 / 11 \\
8 / 7 \\
8 / 21 \\
9 / 5 \\
9 / 18 \\
10 / 4 \\
10 / 19 \\
11 / 2 \\
11 / 18 \\
\end{array}$ & $\begin{array}{l}8.1 \\
3.2 \\
1.5 \\
1.0 \\
2.1 \\
4.1 \\
5.5 \\
8.3 \\
8.3 \\
8.3 \\
4.0 \\
\end{array}$ & $\begin{array}{r}61 \\
63 \\
66 \\
66 \\
63 \\
58 \\
61 \\
50 \\
55 \\
49 \\
45.5 \\
\end{array}$ & $\begin{array}{l}12: 08 \\
13: 08 \\
13: 20 \\
11: 30 \\
14: 45 \\
14: 39 \\
14: 40 \\
10: 35 \\
12: 07 \\
13: 05 \\
13: 21 \\
\end{array}$ & Valley Chapel Rd \\
\hline $\begin{array}{l}\text { West Little Walla } \\
\text { Walla } \\
\end{array}$ & WLW-2 & $\begin{array}{r}5 / 15 \\
6 / 2 \\
\end{array}$ & $\begin{array}{l}2.7 \\
3.0 \\
\end{array}$ & $\begin{array}{l}61 \\
60 \\
\end{array}$ & $\begin{array}{l}12: 13 \\
10: 14 \\
\end{array}$ & Frog Hollow Rd \\
\hline Mud Creek & MC-2 & $\begin{array}{r}5 / 26 \\
6 / 2 \\
6 / 14 \\
6 / 26 \\
7 / 10 \\
8 / 7 \\
8 / 21 \\
9 / 5 \\
9 / 18 \\
10 / 4 \\
10 / 19 \\
11 / 2 \\
11 / 16 \\
\end{array}$ & $\begin{array}{l}1.6 \\
3.7 \\
6.2 \\
2.2 \\
1.5 \\
2.2 \\
7.1 \\
1.6 \\
3.1 \\
0.8 \\
0.1 \\
0.4 \\
4.8 \\
\end{array}$ & $\begin{array}{r}65 \\
64 \\
68 \\
76 \\
77 \\
78 \\
64 \\
59 \\
63.5 \\
52 \\
51 \\
43 \\
36.1 \\
\end{array}$ & $\begin{array}{l}12: 56 \\
11: 35 \\
13: 24 \\
15: 15 \\
15: 00 \\
14: 40 \\
11: 36 \\
11: 30 \\
11: 38 \\
11: 21 \\
08: 40 \\
09: 45 \\
12: 32 \\
\end{array}$ & Barney Rd \\
\hline Pine Creek & PC-1 & $\begin{array}{r}5 / 26 \\
6 / 14 \\
6 / 26 \\
7 / 10 \\
8 / 7 \\
8 / 21 \\
9 / 5 \\
10 / 4 \\
10 / 19 \\
11 / 2 \\
11 / 16\end{array}$ & $\begin{array}{r}7.5 \\
36.1 \\
2.5 \\
1.9 \\
0.1 \\
0.0 \\
-- \\
29.3 \\
1.2 \\
5.6 \\
15.2\end{array}$ & $\begin{array}{r}67 \\
68 \\
76 \\
78 \\
80 \\
65 \\
\\
52 \\
51 \\
43 \\
37.3\end{array}$ & $\begin{array}{l}13: 45 \\
13: 49 \\
15: 30 \\
14: 30 \\
14: 25 \\
11: 23 \\
10: 58 \\
08: 30 \\
09: 45 \\
12: 09\end{array}$ & $\begin{array}{l}\text { Sandpit Rd } \\
\text { No measurable flow }\end{array}$ \\
\hline
\end{tabular}




\section{Appendix C - Stream Temperature Graphs (تF) 2000.}



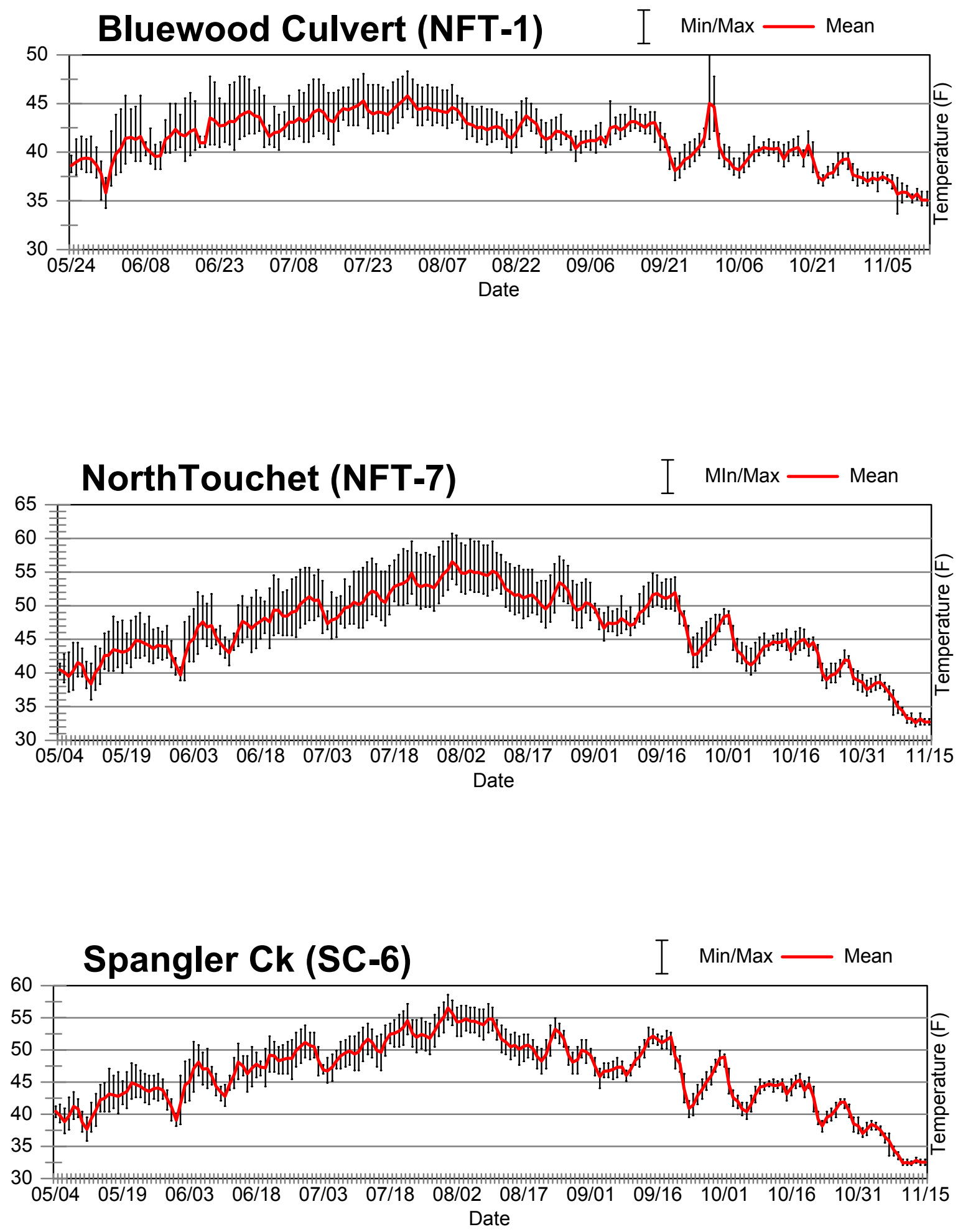

Assessment of Salmonids and Their Habitat Conditions in 

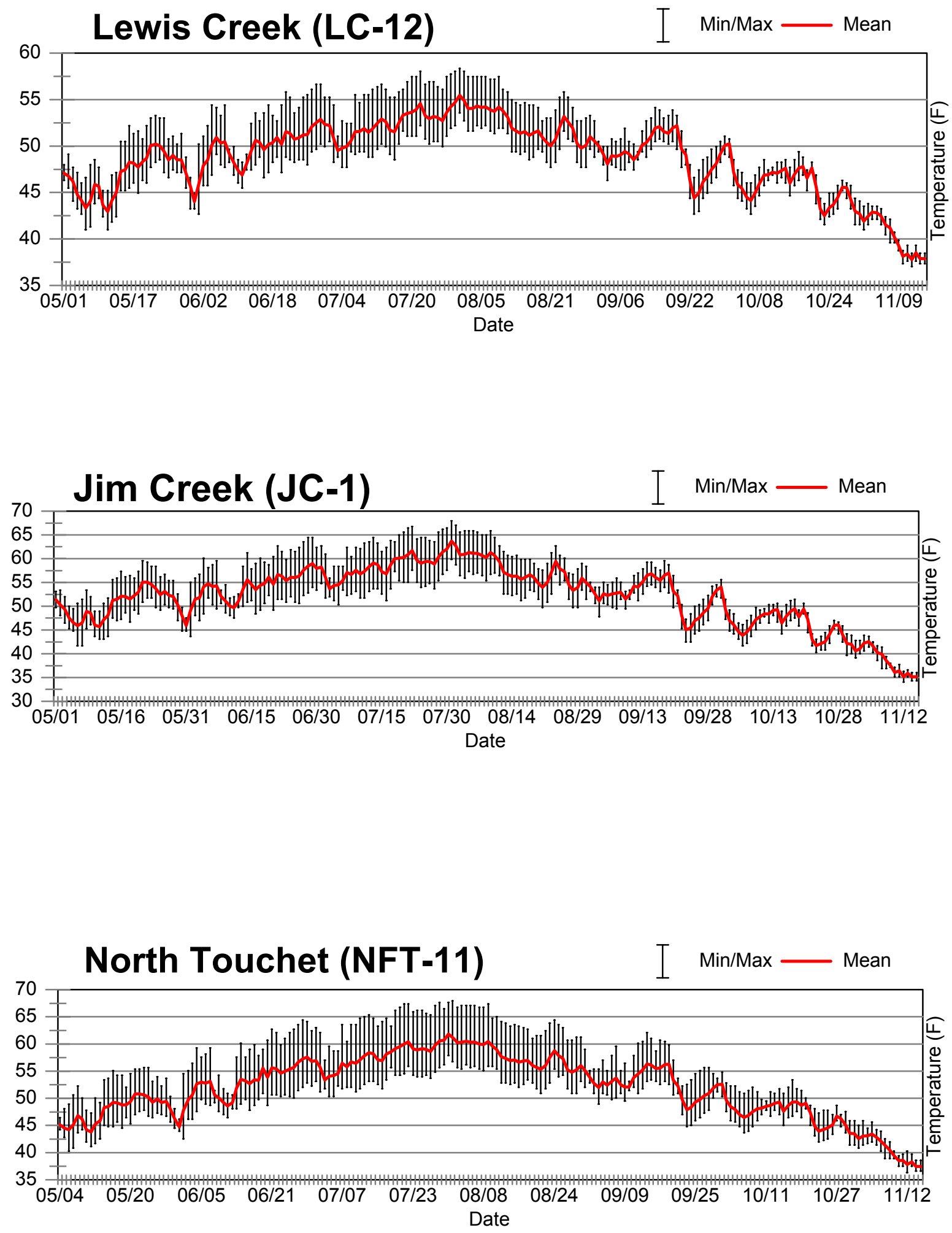

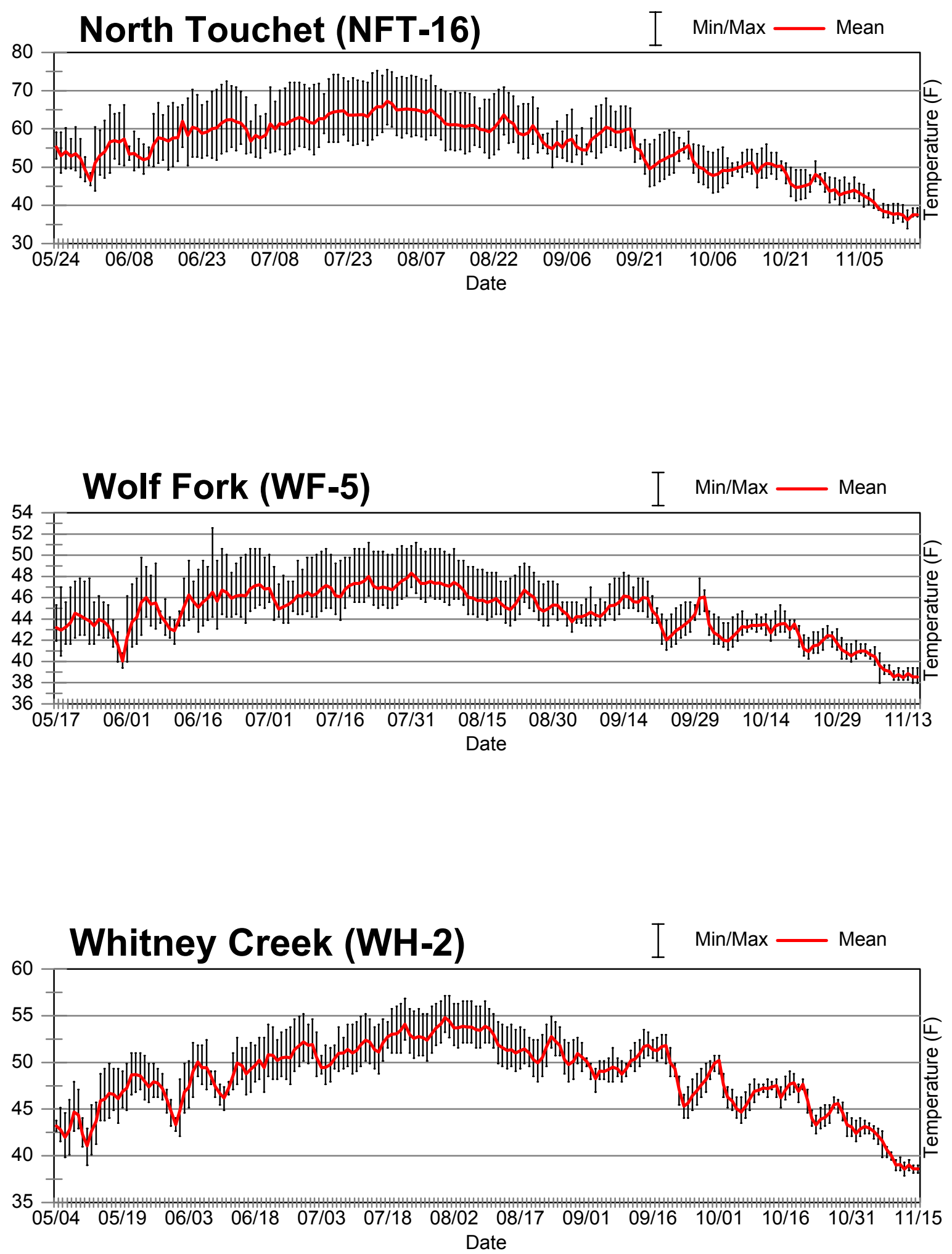

Assessment of Salmonids and Their Habitat Conditions in 

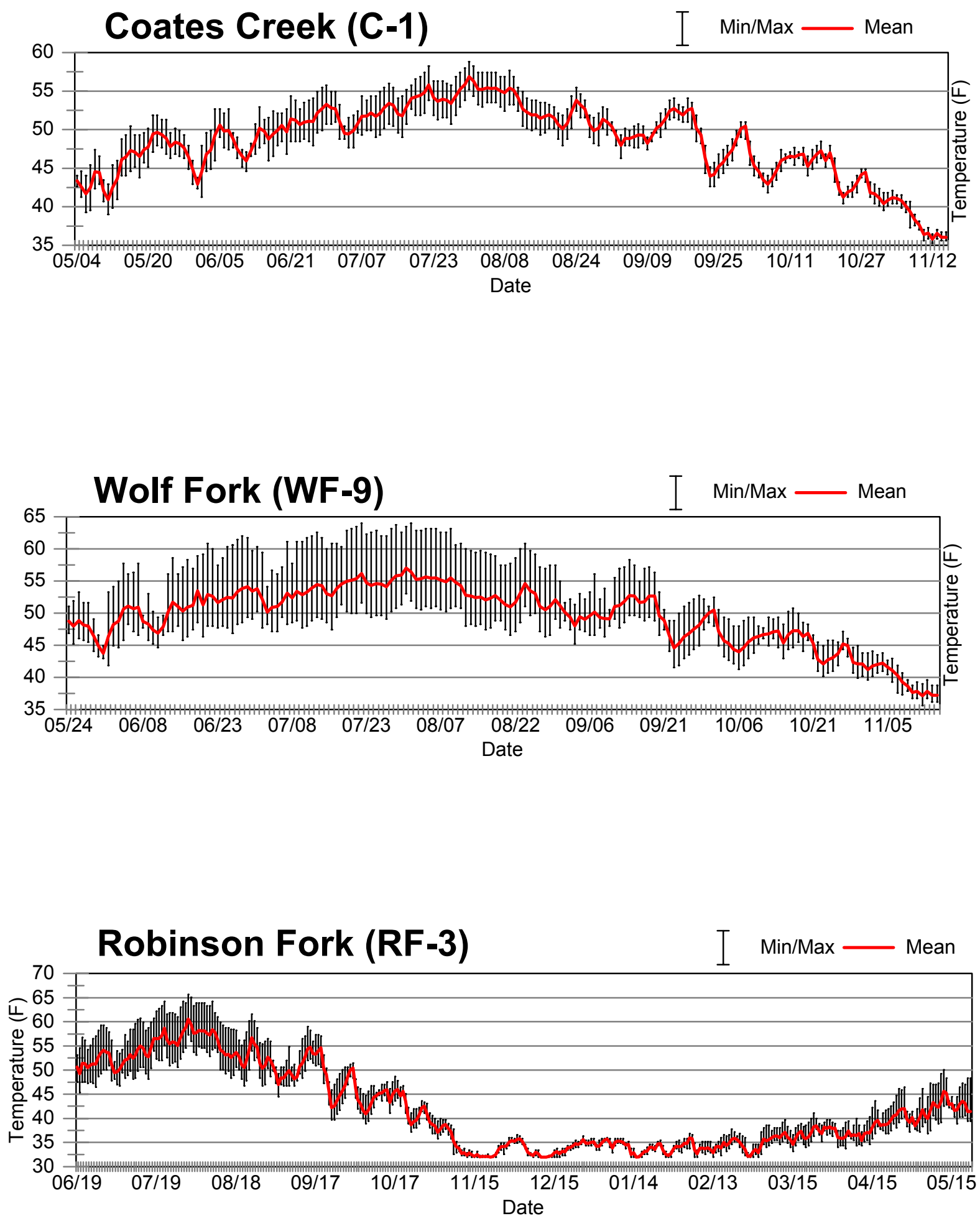

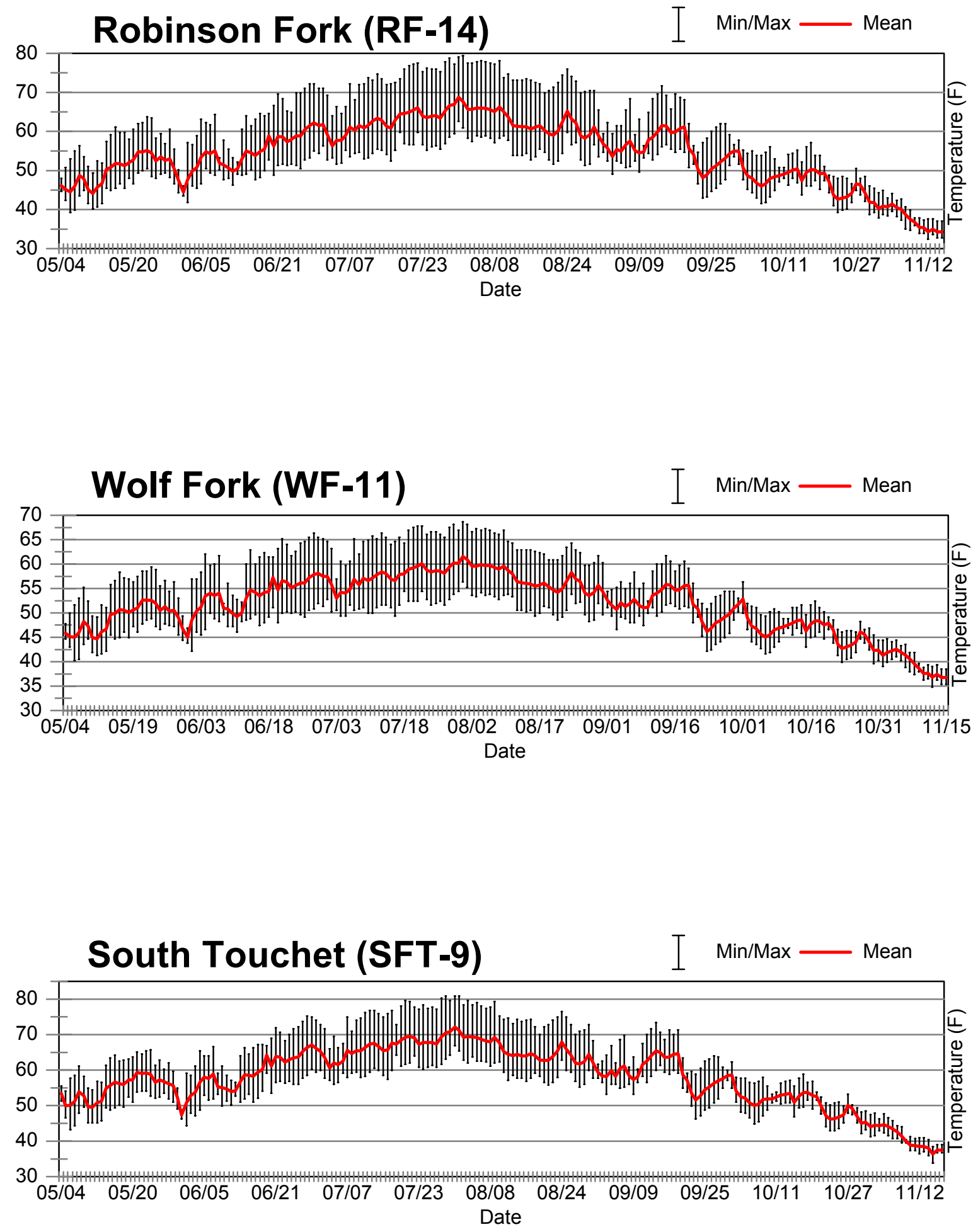

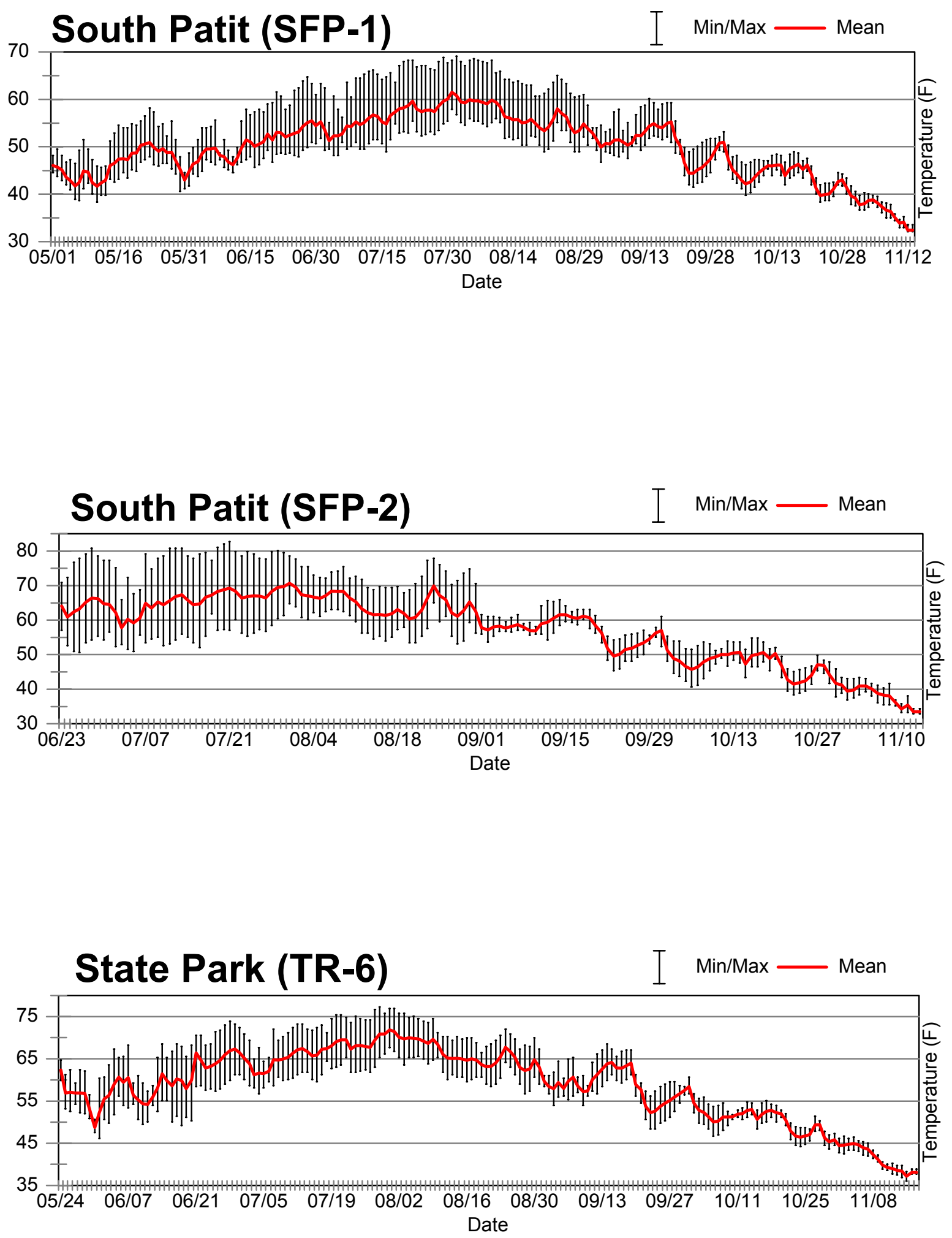
South Coppei (SFC-3) I Min/Max — Mean
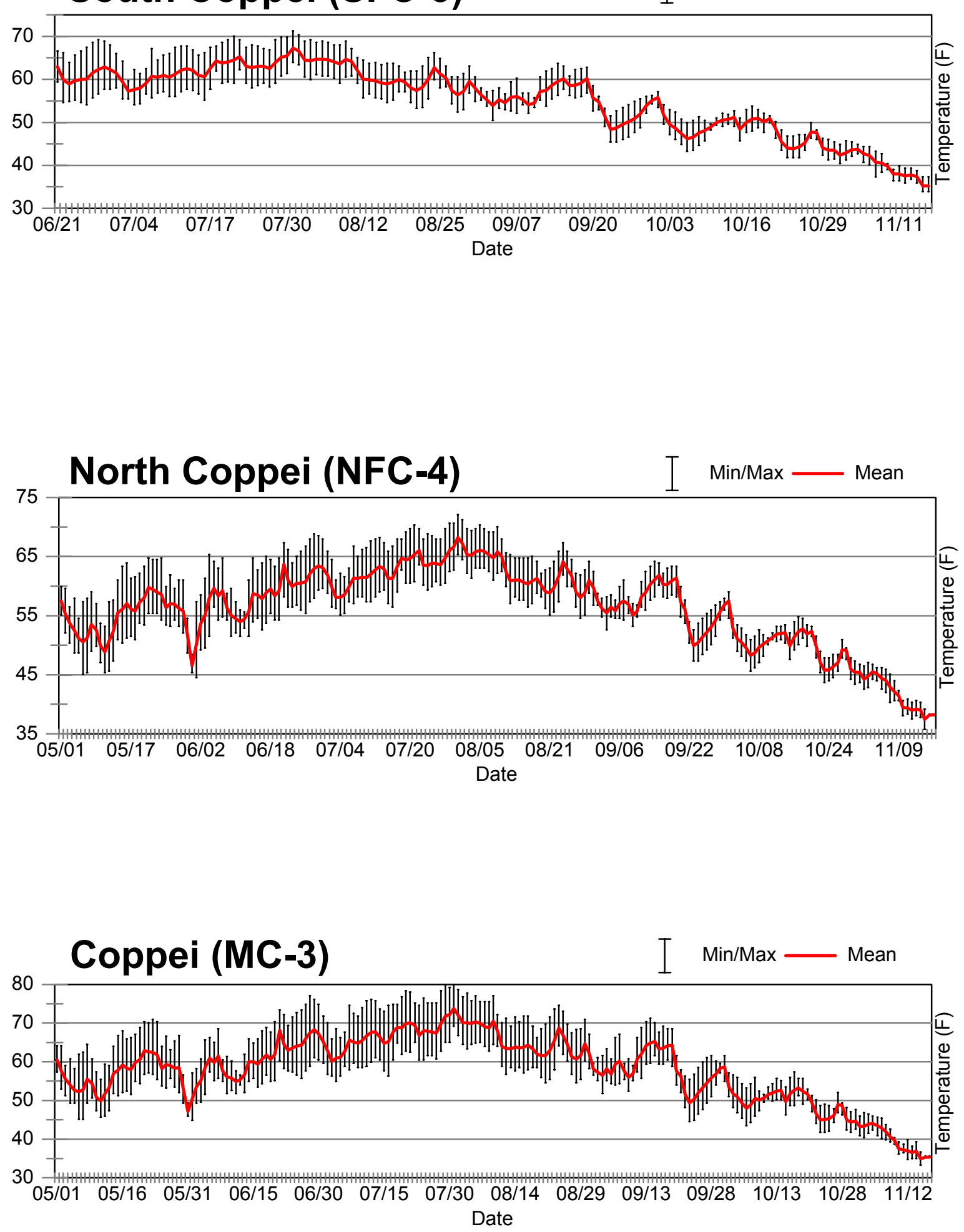

Assessment of Salmonids and Their Habitat Conditions in 

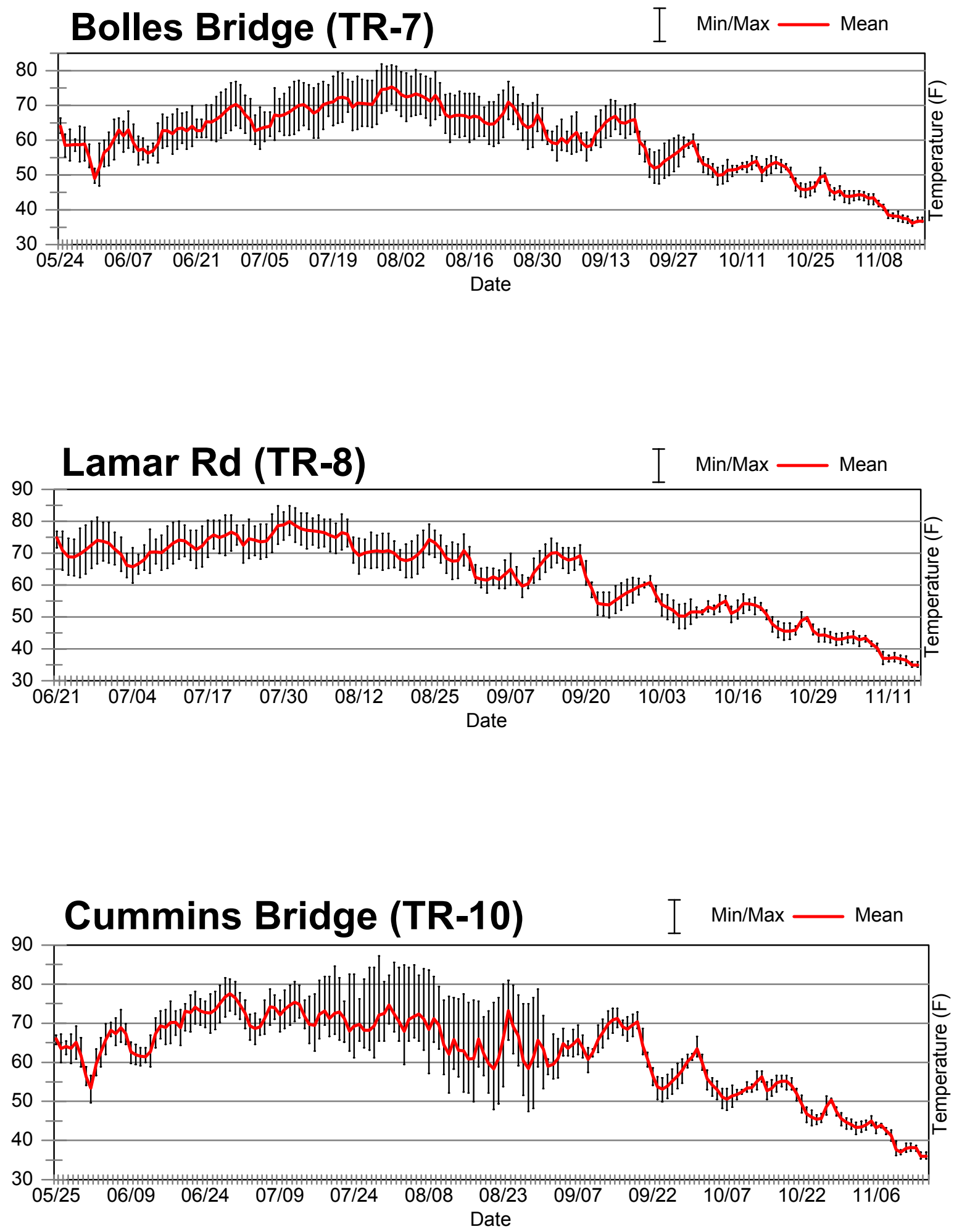

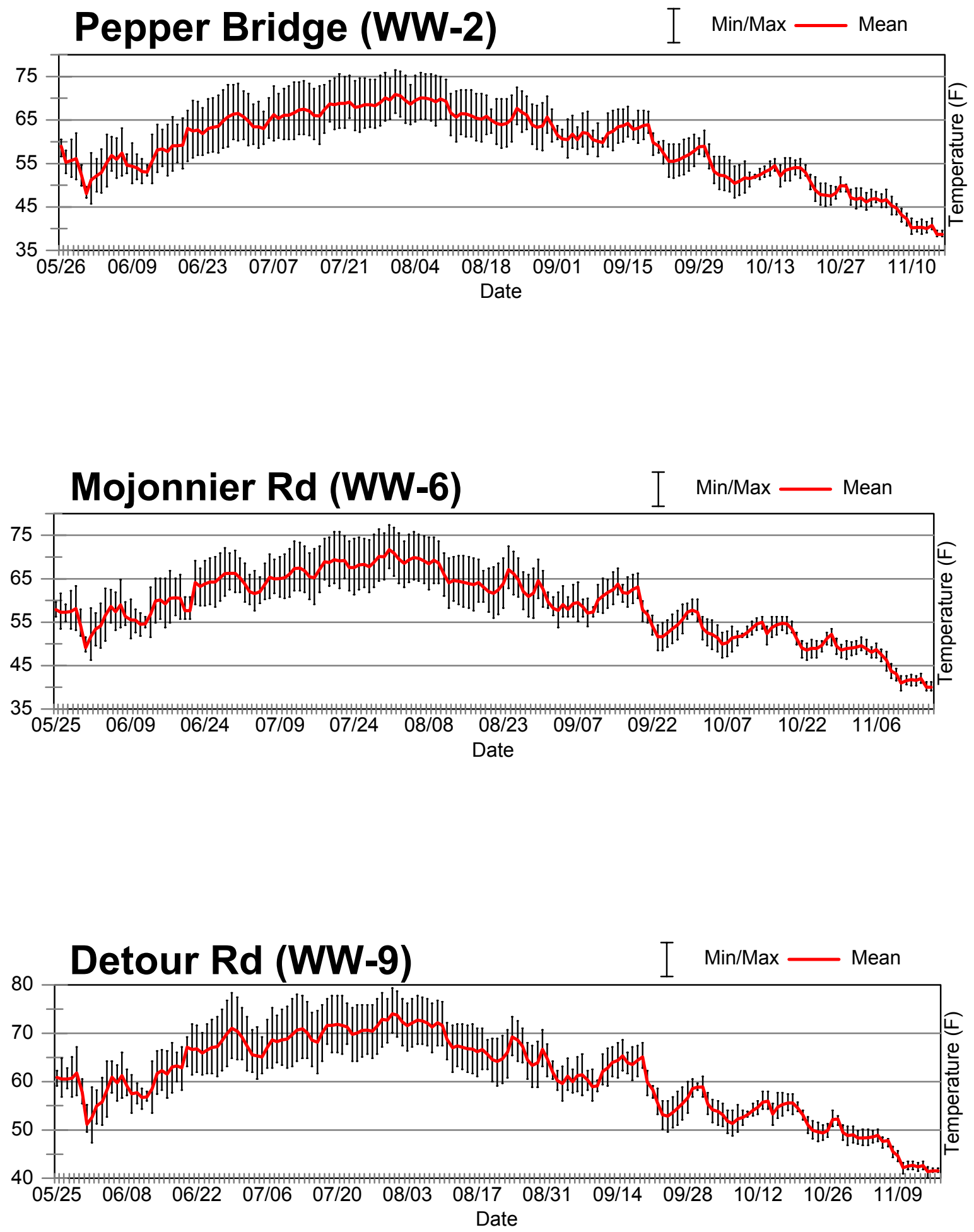

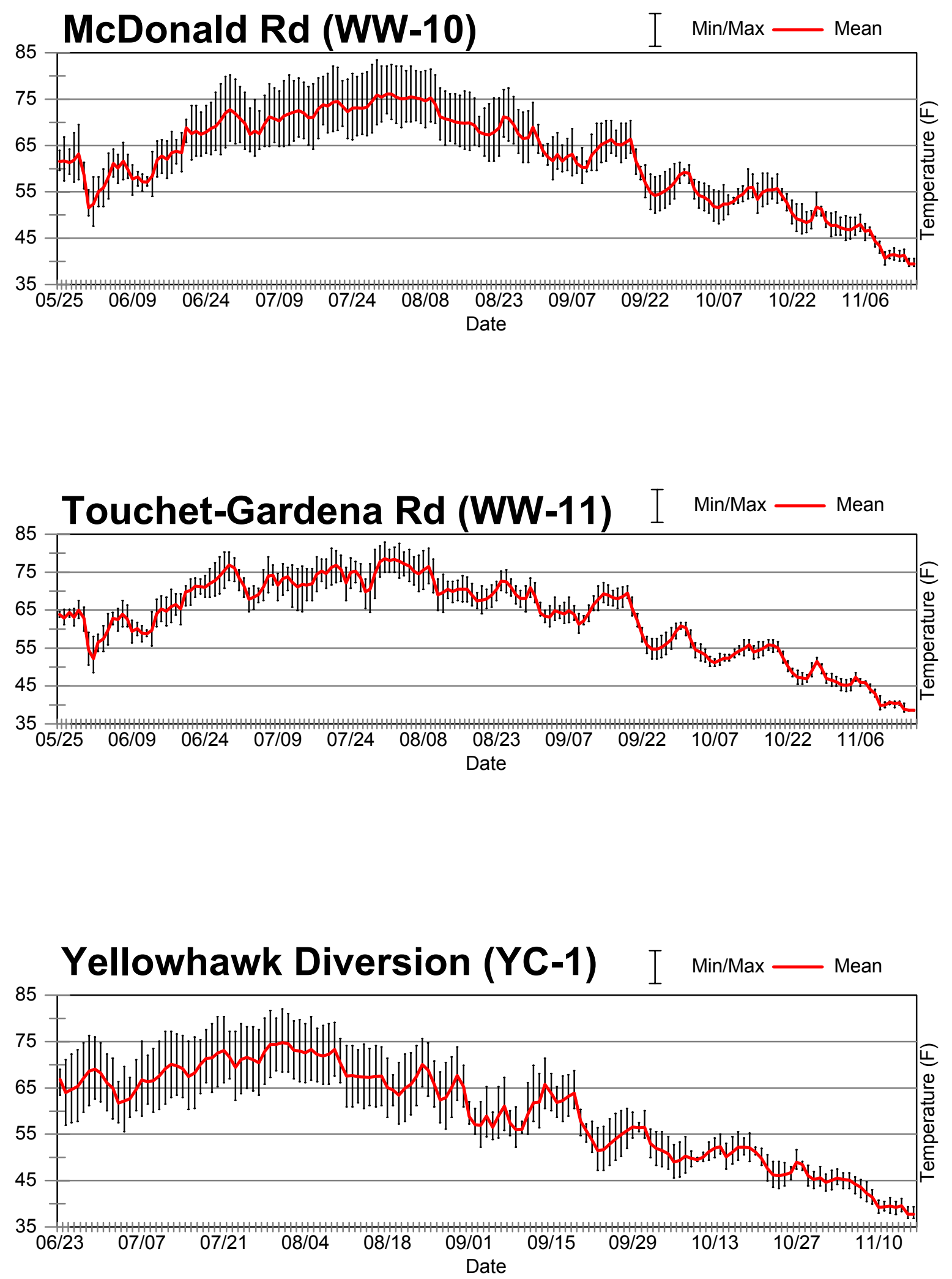

Assessment of Salmonids and Their Habitat Conditions in 

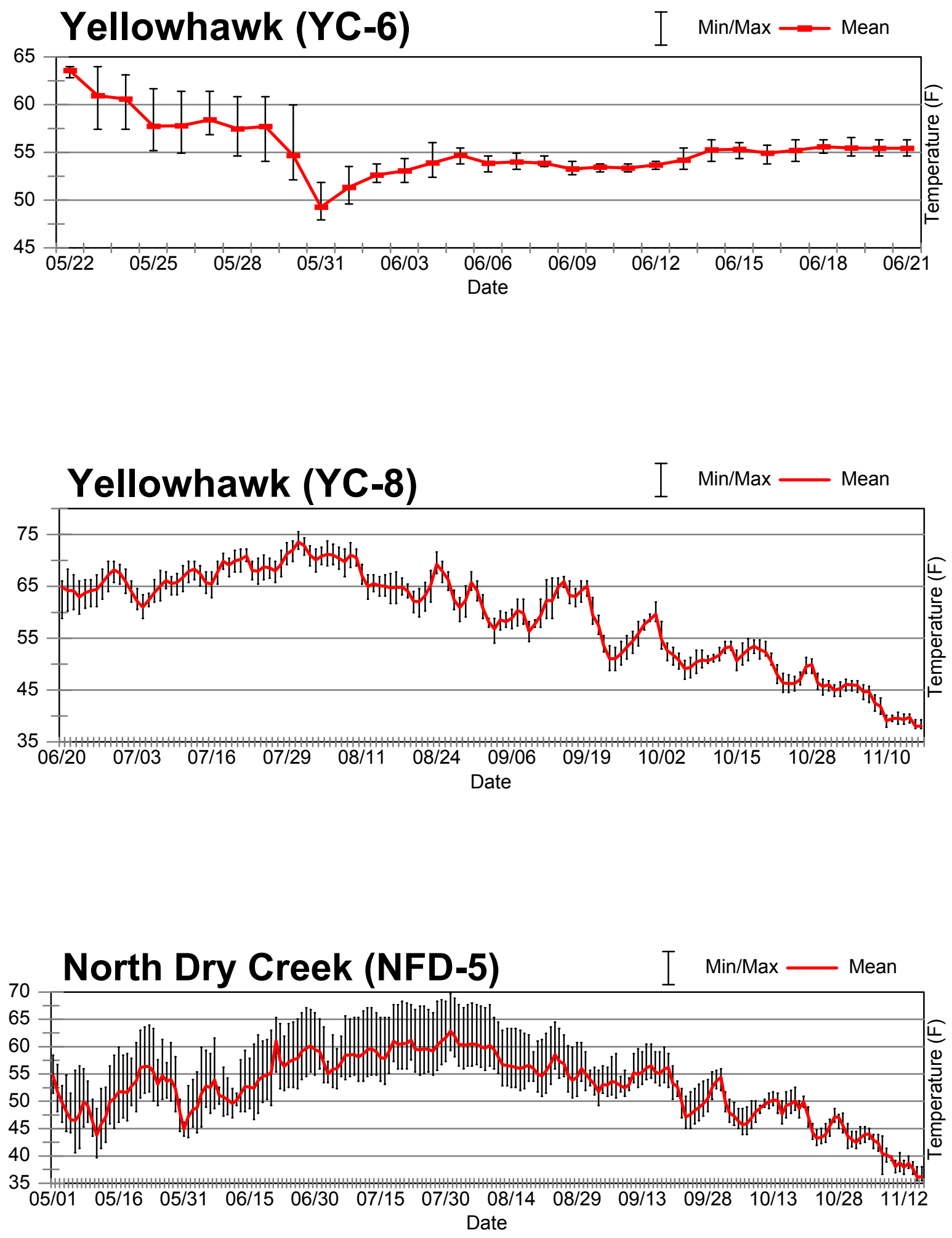

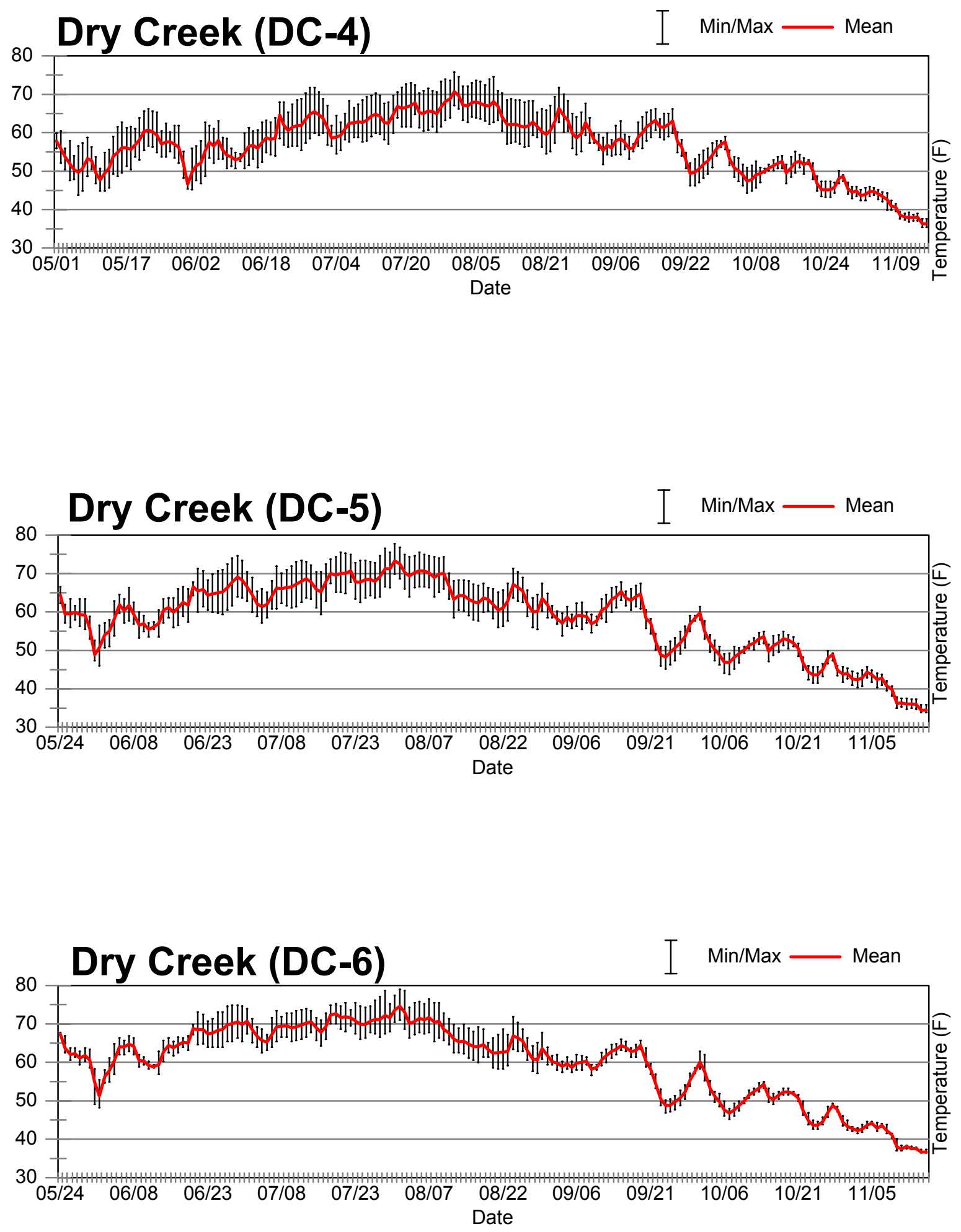


\section{Appendix C1. Additional Stream Temperature Graphs 2000 \\ Temperature data provided by Army Corp of Engineers (Ben Tice) and The Walla Walla Conservation District}




\begin{tabular}{|c|c|c|c|c|}
\hline \multicolumn{5}{|c|}{ COE/WWCD Temperature Monitors, 1999.} \\
\hline Stream Name & Site Name & Type & Location & Comments \\
\hline Birch Creek & Pepper Rd & Temp & T6N, R35E, S13, NW $1 / 4$ & In-7/13/1999 \\
\hline Caldwell Creek & S. Second & Temp & T7N, R36E, S33, SW $1 / 4$ & In-7/13/1999 \\
\hline Cold Creek & Last Chance Rd & Temp & T7N, R35E, S32, NE $1 / 4$ & In-7/13/1999 \\
\hline Cottonwood Creek 1 & Plaza Way & Temp & T6N, R36E, S6, SE $1 / 4$ & In-7/13/1999 \\
\hline Cottonwood Creek 2 & Hood Rd & Temp & T6N, R36E, S11, SW $1 / 4$ & In-7/13/1999 \\
\hline Doan Creek & Whitman Mission & Temp & T7N, R35E, S38, NE $1 / 4$ & In-7/13/1999 \\
\hline East Little Walla Walla & Springdale Rd & Temp & T6N, R35E, S38, SW $1 / 4$ & In-7/13/1999 \\
\hline Garrison Creek 1 & Majonnier & Temp & T6N, R35E, S3, SW1/4 & In-7/13/1999 \\
\hline Garrison Creek 2 & $\mathrm{Pi}-\mathrm{Hi}$ & Temp & T7N, R35E, S38, NE1/4 & In-8/5/1999 \\
\hline Mill Creek 1 & Whitman Mission & Temp & T7N, R36E, S21, SE1/4 & In-8/5/1999 \\
\hline Mill Creek 2 & 5-mile Rd & Temp & T7N, R37E, S18, NE $1 / 4$ & In-8/5/1999 \\
\hline Mud Creek 1 & Barney Rd & Temp & T7N, R34E, S31, SW1/4 & In-7/20/1999 \\
\hline Mud Creek 2 & Private drive off Locher & Temp & T6N, R35E, S7, NE $1 / 4$ & In-7/13/1999 \\
\hline Pine Creek 1 & Sand Pit Rd & Temp & T6N, R33E, S1, NW1/4 & In-7/20/1999 \\
\hline Pine Creek 2 & Stateline Rd & Temp & T6N, R34E, S17, NW1/4 & In-7/20/1999 \\
\hline Reser Creek & & & T7N, R36E, S34, SW1/4 & 7/13/99 Dry \\
\hline Russell Creek 1 & Plaza Way & Temp & T6N, R36E, S5, NW1/4 & In-7/13/1999 \\
\hline Russell Creek 2 & Russell Creek Rd & Temp & T7N, R37E, S29, SW $1 / 4$ & In-7/13/1999 \\
\hline Spring Creek & Rt 12 bridge & Temp & T7N, R37E, S5, NW1/4 & In-7/13/1999 \\
\hline Stone Creek & Tiatan,@3rd & Temp & T7N, R36E, S29, SW1/4 & In-7/13/1999 \\
\hline Titus Creek & 5-mile Rd & Temp & T7N, R37E, S18, NE $1 / 4$ & In-7/13/1999 \\
\hline West Little Walla Walla & Stoval Rd & Temp & T7N, R35E, S38, SW1/4 & In-7/13/1999 \\
\hline
\end{tabular}



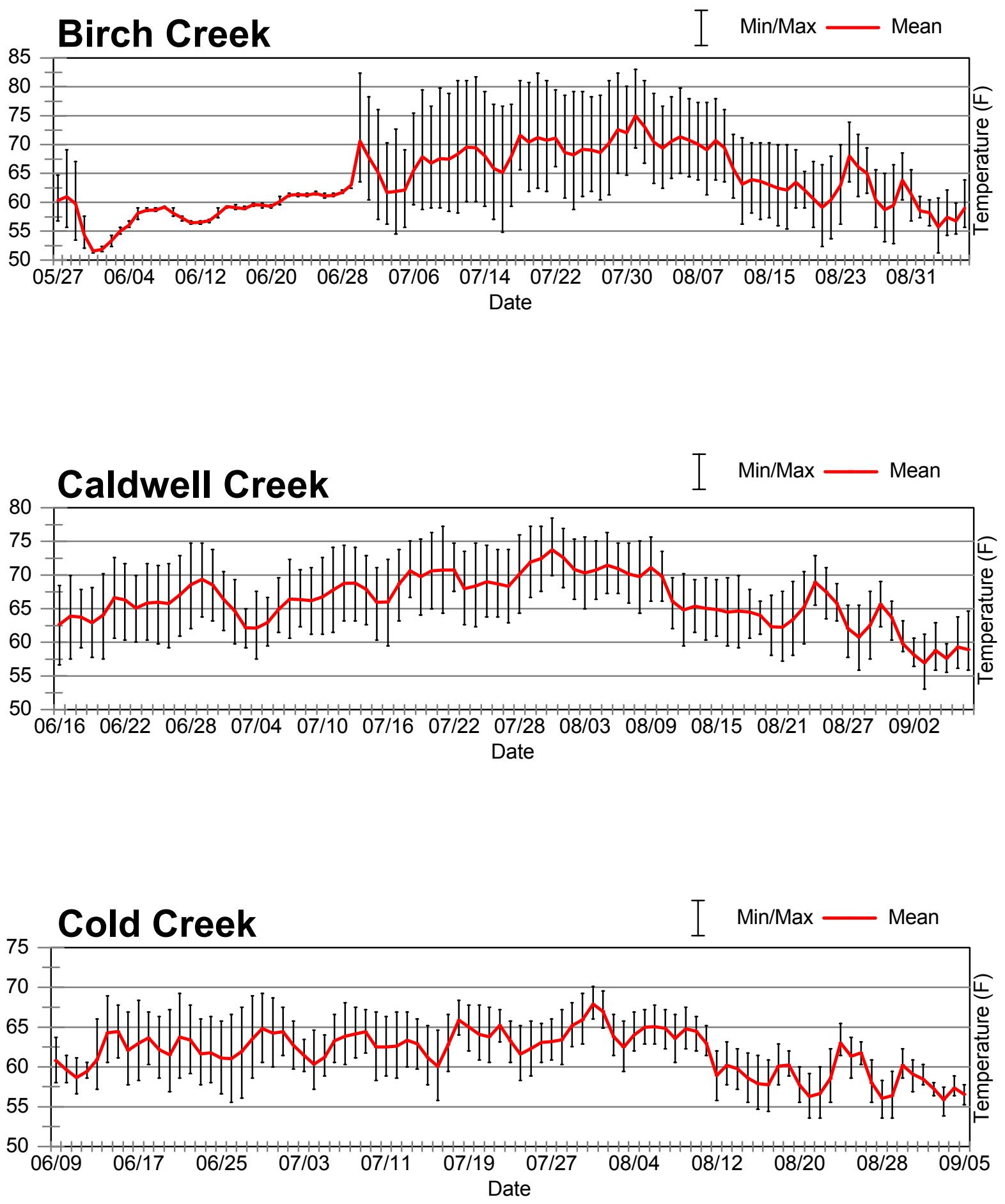

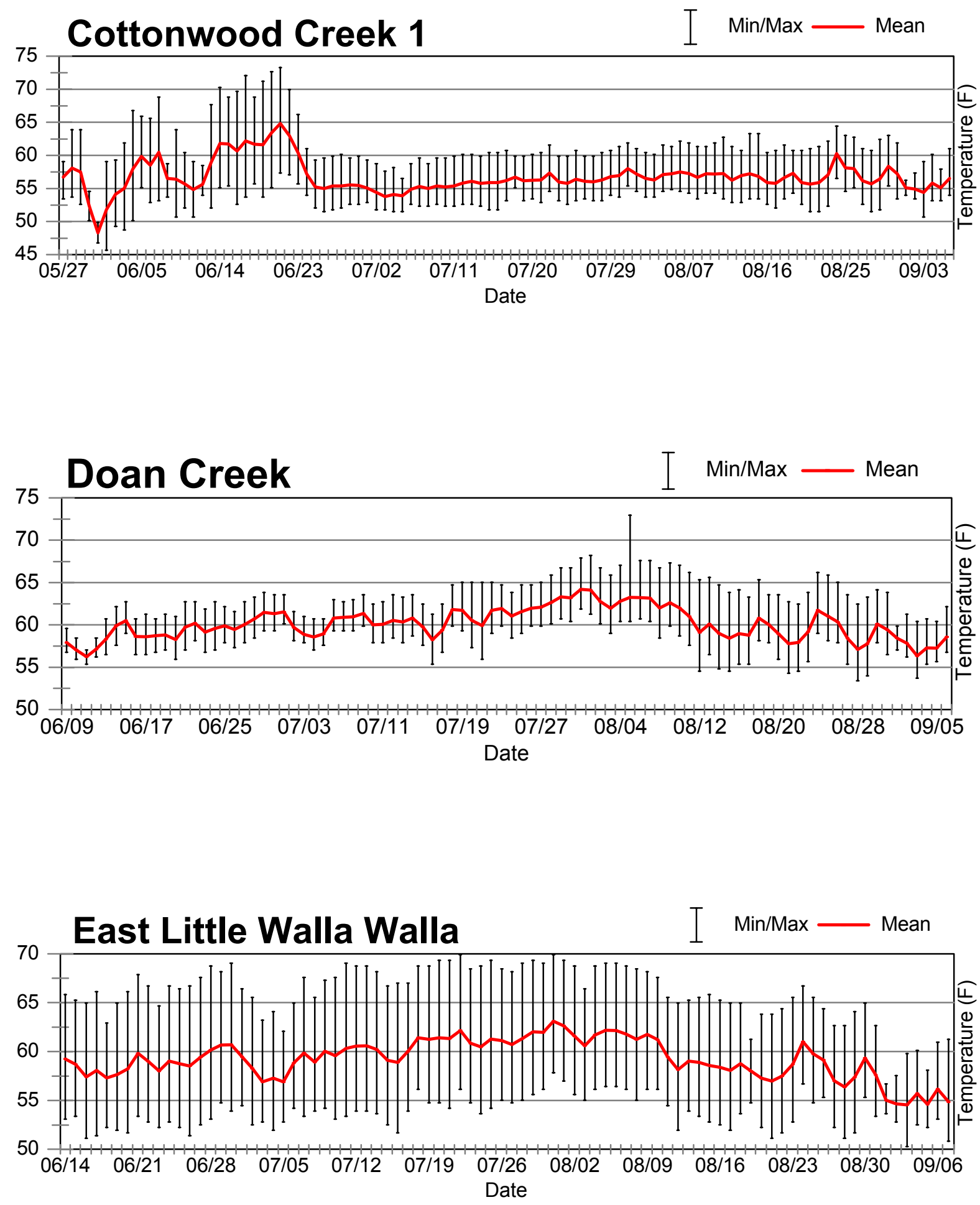

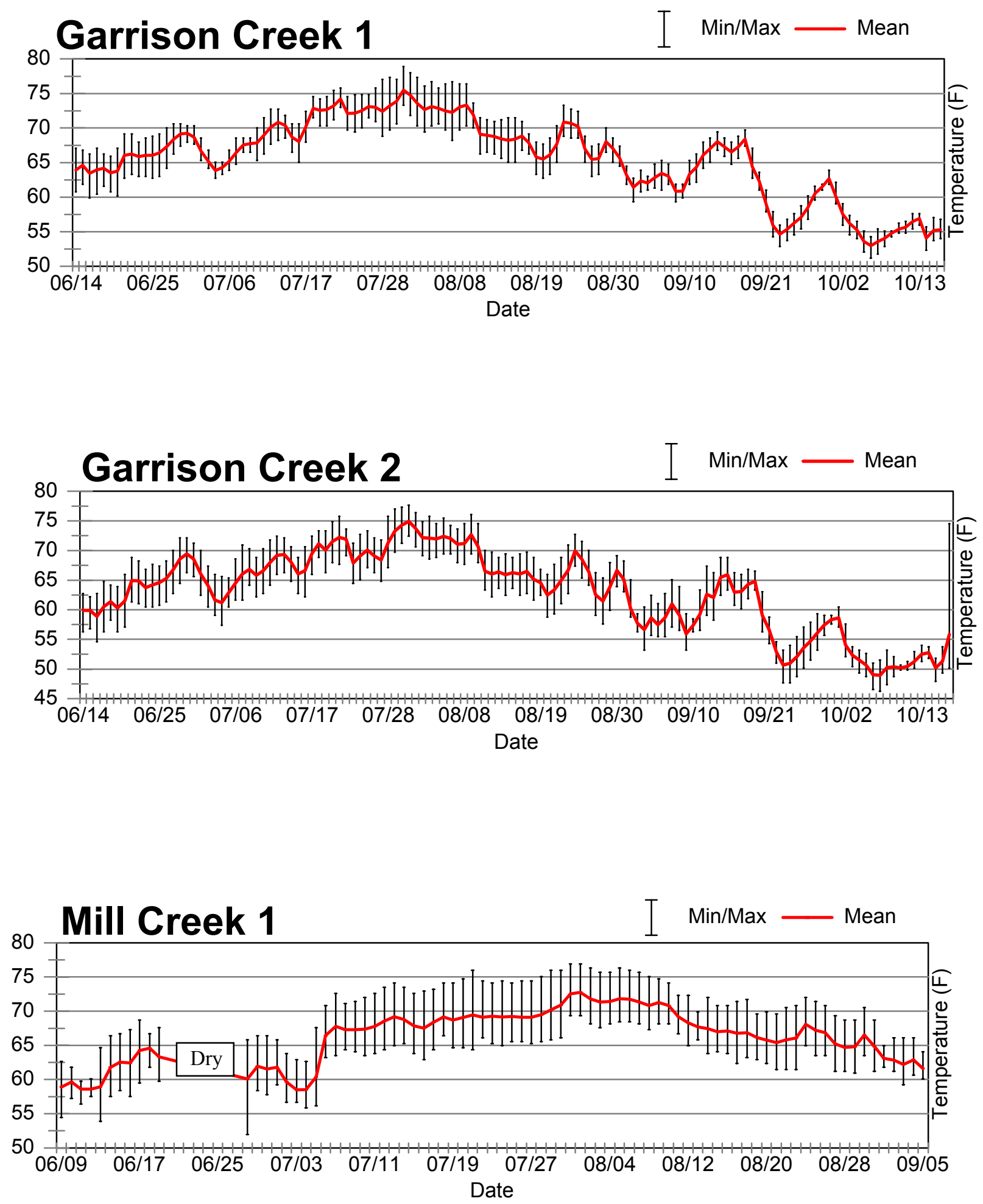

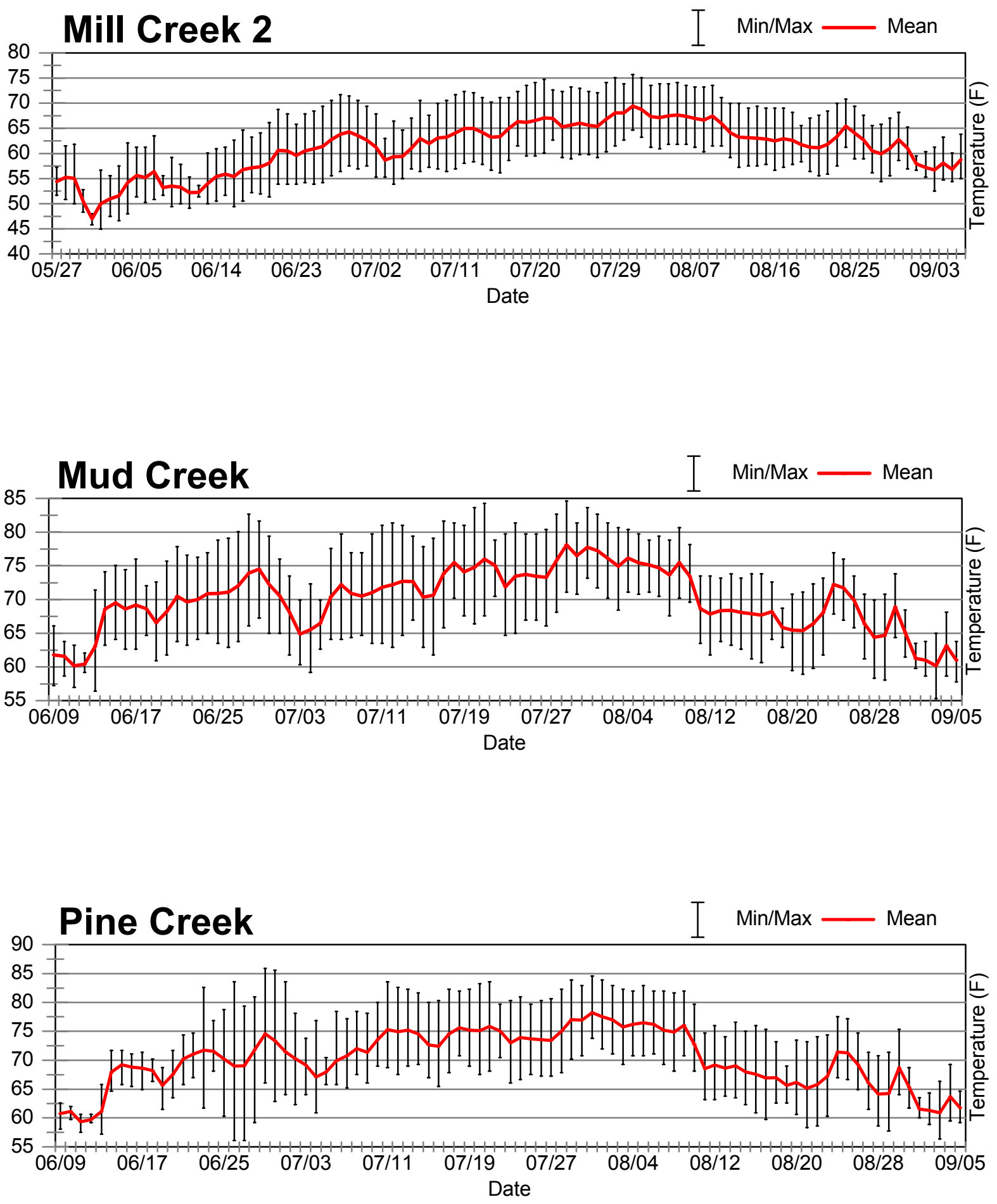

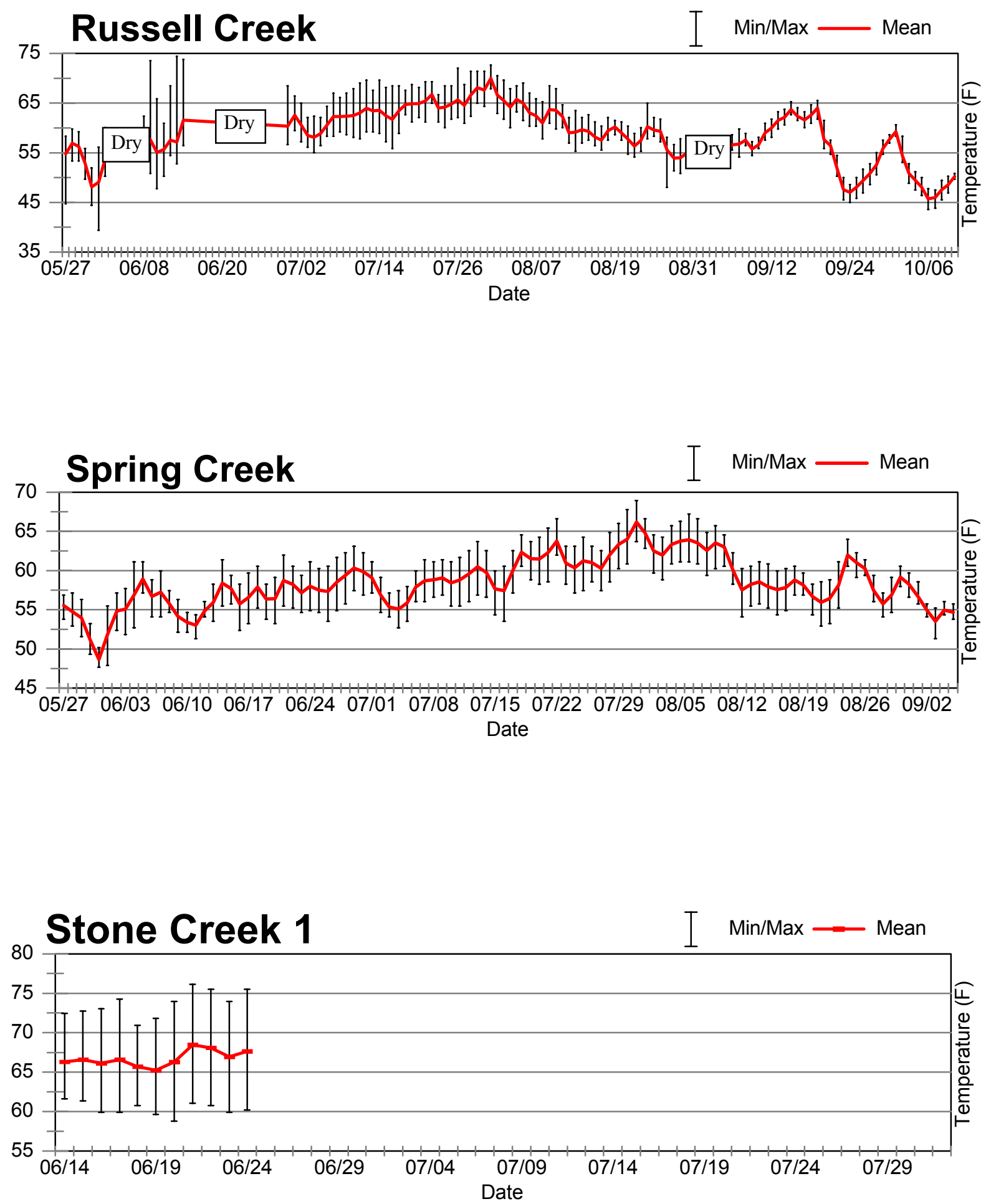

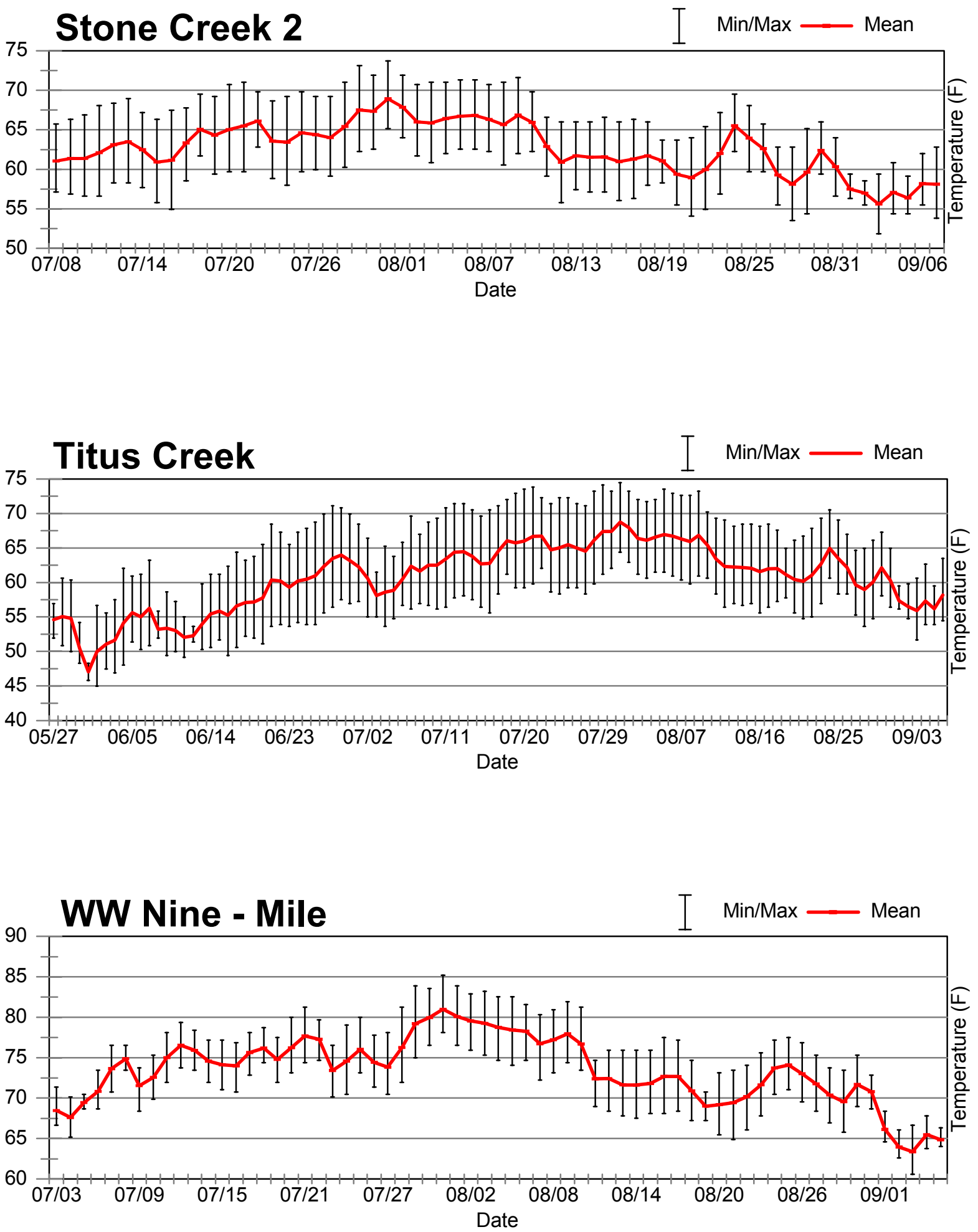

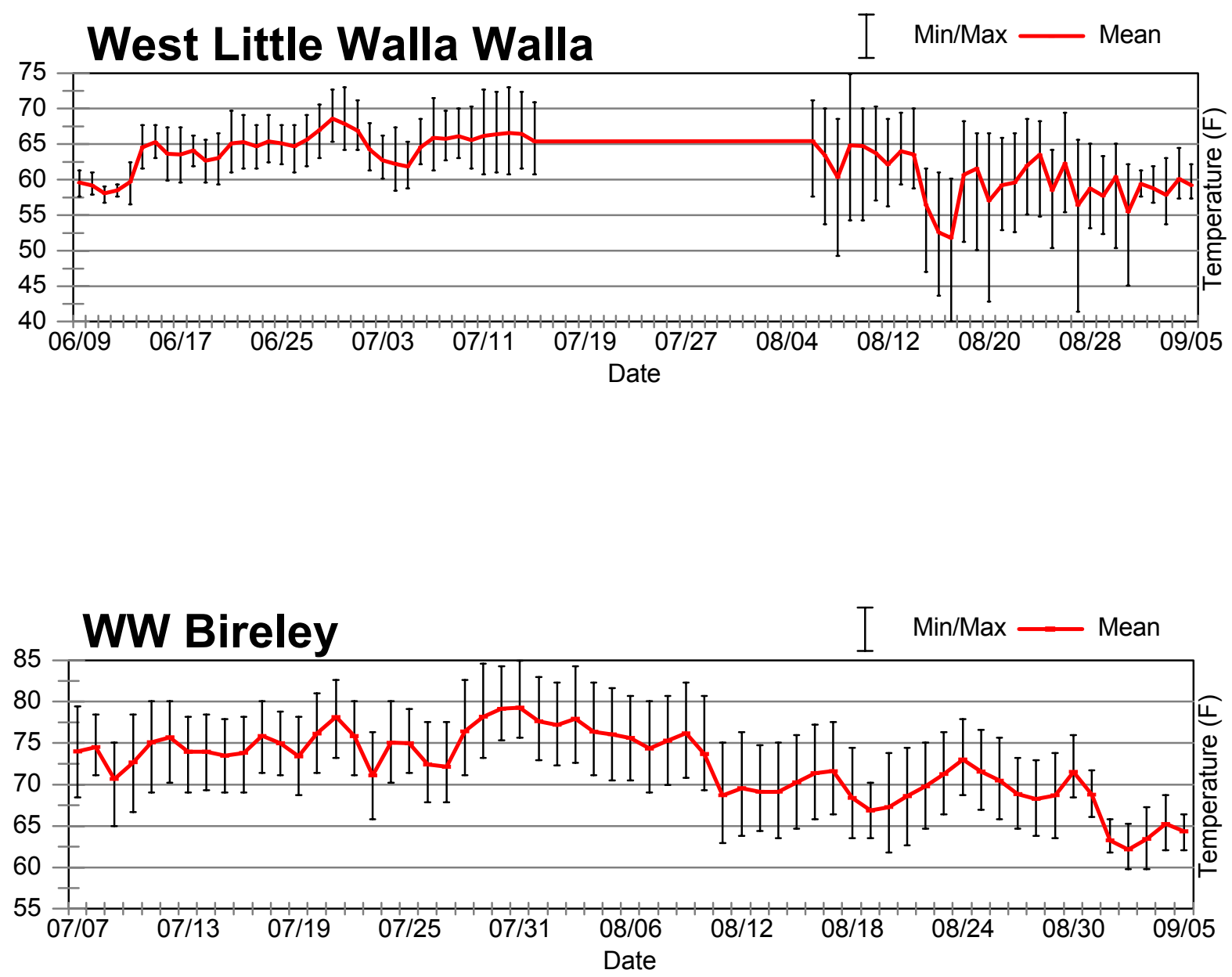


\section{Appendix D. Water Quality Data 2000}

Assessment of Salmonids and Their Habitat Conditions in 


\begin{tabular}{|c|c|c|c|c|}
\hline Data & Stream & Site \# & pH & Conductivity (umhos/cm) \\
\hline $6-27-00$ & SF Patit & SFP-1 & $\begin{array}{l}7.07 \\
\text { Temp (C) } 10.9 \\
\text { Time 9:30 }\end{array}$ & $\begin{array}{l}74.0 \\
\text { Temp 11.4 (C) } \\
\text { Time 9:30 }\end{array}$ \\
\hline $6-27-00$ & Spangler Ck & SC-6 & $\begin{array}{l}7.05 \\
\text { Temp (C) } 11.5 \\
\text { Time 10:52 }\end{array}$ & $\begin{array}{l}52.7 \\
\text { Temp (C) } 12.1 \\
\text { Time 10:52 }\end{array}$ \\
\hline $6-27-00$ & NF Touchet & NFT-7 & $\begin{array}{l}7.10 \\
\text { Temp (C) } 11.5 \\
\text { Time 11:17 }\end{array}$ & $\begin{array}{l}53.3 \\
\text { Temp (C) } 11.9 \\
\text { Time 11:17 }\end{array}$ \\
\hline $6-27-00$ & NF Touchet & NFT-11 & $\begin{array}{l}7.48 \\
\text { Temp (C) } 16.8 \\
\text { Time 12:26 }\end{array}$ & $\begin{array}{l}63.6 \\
\text { Temp (C) } 17.3 \\
\text { Time 12:26 }\end{array}$ \\
\hline $6-29-00$ & NF Touchet & NFT-16 & $\begin{array}{l}7.11 \\
\text { Temp (C) } 15.5 \\
\text { Time 9:25 }\end{array}$ & $\begin{array}{l}83.1 \\
\text { Temp (C) } 15.7 \\
\text { Time 9:25 }\end{array}$ \\
\hline $6-27-00$ & Lewis $\mathrm{Ck}$ & $\mathrm{LC}-12$ & $\begin{array}{l}7.35 \\
\text { Temp (C) } 13.1 \\
\text { Time - - }\end{array}$ & $\begin{array}{l}72.6 \\
\text { Temp (C) } 14.1 \\
\text { Time - - }\end{array}$ \\
\hline $6-27-00$ & $\mathrm{Jim}$ Ck & $\mathrm{JC}-1$ & $\begin{array}{l}7.55 \\
\text { Temp (C) } 17.0 \\
\text { Time - - }\end{array}$ & $\begin{array}{l}92.4 \\
\text { Temp (C) } 17.7 \\
\text { Time - - }\end{array}$ \\
\hline $6-27-00$ & Whitney Ck & WH-2 & $\begin{array}{l}7.23 \\
\text { Temp (C) } 13.8 \\
\text { Time 13:00 }\end{array}$ & $\begin{array}{l}64.3 \\
\text { Temp (C) } 14.1 \\
\text { Time 13:00 }\end{array}$ \\
\hline $6-27-00$ & Coates $\mathrm{Ck}$ & $\mathrm{C}-1$ & $\begin{array}{l}7.46 \\
\text { Temp (C) } 13.8 \\
\text { Time 13:26 }\end{array}$ & $\begin{array}{l}62.5 \\
\text { Temp (C) } 14.1 \\
\text { Time 13:26 }\end{array}$ \\
\hline $6-27-00$ & Wolf Fork & WF-9 & $\begin{array}{l}7.69 \\
\text { Temp (C) } 16.3 \\
\text { Time 13:53 }\end{array}$ & $\begin{array}{l}61.5 \\
\text { Temp (C) } 17.0 \\
\text { Time 13:53 }\end{array}$ \\
\hline $6-27-00$ & Wolf Fork & WF-11 & $\begin{array}{l}7.84 \\
\text { Temp (C) } 19.5 \\
\text { Time 14:50 }\end{array}$ & $\begin{array}{l}62.0 \\
\text { Temp (C) } 19.8 \\
\text { Time 14:50 }\end{array}$ \\
\hline $6-27-00$ & Robinson Fork & RF-14 & $\begin{array}{l}7.63 \\
\text { Temp (C) } 21.2 \\
\text { Time 14:15 }\end{array}$ & $\begin{array}{l}58.7 \\
\text { Time (C) } 21.7 \\
\text { Time 14:15 }\end{array}$ \\
\hline $6-29-00$ & SF Touchet & SFT-9 & $\begin{array}{l}7.28 \\
\text { Temp (C) } 17.9 \\
\text { Time 9:45 }\end{array}$ & $\begin{array}{l}108.7 \\
\text { Time (C) } 17.9 \\
\text { Time 9:45 }\end{array}$ \\
\hline
\end{tabular}




\begin{tabular}{|c|c|c|c|c|}
\hline Data & Stream & Site \# & pH & Conductivity (umhos/cm) \\
\hline $6-29-00$ & Touchet R & TR-4 & $\begin{array}{l}7.7 \\
\text { Temp (C) } 17.6 \\
\text { Time 10:20 }\end{array}$ & $\begin{array}{l}113.5 \\
\text { Temp (C) } 17.8 \\
\text { Time 10:20 }\end{array}$ \\
\hline $6-29-00$ & Touchet R & TR-7 & $\begin{array}{l}9.15 \\
\text { Temp (C) } 21.2 \\
\text { Time 10:55 }\end{array}$ & $\begin{array}{l}111.5 \\
\text { Temp (C) } 21.2 \\
\text { Time 10:55 }\end{array}$ \\
\hline $6-29-00$ & Touchet R & TR-9 & $\begin{array}{l}8.43 \\
\text { Temp (C) } 24.2 \\
\text { Time 11:50 }\end{array}$ & $\begin{array}{l}103.7 \\
\text { Temp (C) } 24.4 \\
\text { Time 11:50 }\end{array}$ \\
\hline $7-10-00$ & Touchet R & TR-9 & $\begin{array}{l}9.09 \\
\text { Temp (C) } 24.6 \\
\text { Time 13:25 }\end{array}$ & $\begin{array}{l}80.4 \\
\text { Temp (C) } 24.7 \\
\text { Time 13:25 }\end{array}$ \\
\hline $6-29-00$ & Touchet R & TR-10 & $\begin{array}{l}7.98 \\
\text { Temp (C) } 25.5 \\
\text { Time 12:20 }\end{array}$ & $\begin{array}{l}137.1 \\
\text { Temp (C) } 25.6 \\
\text { Time 12:20 }\end{array}$ \\
\hline $7-10-00$ & Touchet R & TR-10 & $\begin{array}{l}8.54 \\
\text { Temp (C) } 23.8 \\
\text { Time 14:00 }\end{array}$ & $\begin{array}{l}89.0 \\
\text { Temp (C) } 24.4 \\
\text { Time 14:00 }\end{array}$ \\
\hline $6-29-00$ & Walla Walla & WW-9 & $\begin{array}{l}8.78 \\
\text { Temp (C) } 25.2 \\
\text { Time 13:10 }\end{array}$ & $\begin{array}{l}229.0 \\
\text { Temp (C) } 25.3 \\
\text { Time 13:10 }\end{array}$ \\
\hline $7-10-00$ & Walla Walla & WW-9 & $\begin{array}{l}9.31 \\
\text { Temp (C) } 24.6 \\
\text { Time 16:00 }\end{array}$ & $\begin{array}{l}155.1 \\
\text { Temp (C) } 24.9 \\
\text { Time 16:00 }\end{array}$ \\
\hline $6-29-00$ & Walla Walla & WW-6 & $\begin{array}{l}8.26 \\
\text { Temp (C) } 22.5 \\
\text { Time 13:40 }\end{array}$ & $\begin{array}{l}125.5 \\
\text { Temp (C) } 22.7 \\
\text { Time 13:40 }\end{array}$ \\
\hline $6-29-00$ & Walla Walla & WW-2 & $\begin{array}{l}8.05 \\
\text { Temp (C) } 23.6 \\
\text { Time 14:30 }\end{array}$ & $\begin{array}{l}117.4 \\
\text { Temp (C) } 23.7 \\
\text { Time 14:30 }\end{array}$ \\
\hline $7-10-00$ & Walla Walla & WW-10 & $\begin{array}{l}9.46 \\
\text { Temp (C) } 28.7 \\
\text { Time 15:20 }\end{array}$ & $\begin{array}{l}170.2 \\
\text { Temp (C) } 29.0 \\
\text { Time 15:20 }\end{array}$ \\
\hline $7-10-00$ & Walla Walla & WW-8 & $\begin{array}{l}8.6 \\
\text { Temp (C) } 23.4 \\
\text { Time 16:15 }\end{array}$ & $\begin{array}{l}290 \\
\text { Temp (C) } 23.6 \\
\text { Time 16:15 }\end{array}$ \\
\hline
\end{tabular}




\begin{tabular}{|c|c|c|c|c|}
\hline Data & Stream & Site \# & pH & Conductivity (umhos/cm) \\
\hline $7-10-00$ & Pine $\mathrm{Ck}$ & PC-1 & $\begin{array}{l}8.76 \\
\text { Temp (C) } 24.4 \\
\text { Time 14:30 }\end{array}$ & $\begin{array}{l}354 \\
\text { Temp (C) } 25.6 \\
\text { Time 14:30 }\end{array}$ \\
\hline $7-10-00$ & Mud Ck & MC-2 & $\begin{array}{l}7.89 \\
\text { Temp (C) } 24.8 \\
\text { Time 15:00 }\end{array}$ & $\begin{array}{l}239 \\
\text { Temp (C) } 25.1 \\
\text { Time 15:00 }\end{array}$ \\
\hline
\end{tabular}




\section{Appendix E. Qualitative Electrofishing 2000}


Relative abundance for qualitative electrofishing sites in the Walla Walla River basin, 2000.

\begin{tabular}{|c|c|c|c|c|c|}
\hline Stream & Site \# & Date & $\begin{array}{l}\text { Approx Site } \\
\text { Length (m) } \\
\end{array}$ & Relative Abundance* & Comments \\
\hline \multirow[t]{4}{*}{ NF Touchet } & NFT-2 & $9 / 20$ & 100 & $\begin{array}{l}\text { Three } 1+\text { RBT's }(170-175 \mathrm{~mm}) \\
\& \text { three BT }(50-163 \mathrm{~mm}) \text { found } \\
\text { Tailed-frogs - common }\end{array}$ & $\begin{array}{l}\text { Moderate intensity survey, } \\
\text { sampling mostly pools. }\end{array}$ \\
\hline & NFT-3 & $8 / 29$ & 25 & $\begin{array}{l}\text { One } 55 \mathrm{~mm} \text { BT \& one } 28 \mathrm{~mm} \\
\text { RBT found; RBT \& BT - rare }\end{array}$ & $\begin{array}{l}\text { Moderate intensity survey. } \\
\text { Very few fish found }\end{array}$ \\
\hline & NFT-4 & $9 / 20$ & 100 & $\begin{array}{l}\text { Three BT }(55-63 \mathrm{~mm}) \text { found; } \\
\text { no other fish found }\end{array}$ & $\begin{array}{l}\text { Light survey effort, sampled } \\
\text { mainly pools }\end{array}$ \\
\hline & NFT-5 & $9 / 20$ & 100 & $\begin{array}{l}\text { Five } 0+(35-73 \mathrm{~mm}) \text {, one } 152 \\
\mathrm{~mm} \& \text { one } 215 \mathrm{~mm} \text { RBT found; } \\
\text { four BT }(55-70 \mathrm{~mm}) \text { found }\end{array}$ & $\begin{array}{l}\text { Light survey effort, sampled } \\
\text { mostly pools }\end{array}$ \\
\hline \multirow[t]{3}{*}{ Coppei Ck } & $\mathrm{MC}-1 *$ & $8 / 17$ & 45 & $\begin{array}{l}\text { Five 1+ RBT's for DNA, RBT, } \\
\text { SCP, dace - common }\end{array}$ & $\begin{array}{l}\text { Light effort, mainly } \\
\text { pools electrofished }\end{array}$ \\
\hline & $\mathrm{MC}-3 *$ & $8 / 17$ & 100 & $\begin{array}{l}\text { Five 1+ RBT's for DNA; two } \\
\text { age classes present. RSS, } \\
\text { SCP, BLS, dace - common }\end{array}$ & $\begin{array}{l}\text { Light effort with mainly } \\
\text { pools electrofished. Few } \\
\text { RBT's present for large area }\end{array}$ \\
\hline & $\mathrm{MC}-4^{*}$ & $8 / 17$ & 100 & $\begin{array}{l}\text { Two RBT's ( } 133,135 \mathrm{~mm}) \text {, } \\
\text { RSS \& crayfish - common, } \\
\text { dace - abundant. }\end{array}$ & $\begin{array}{l}\text { Light effort - mainly pools } \\
\text { electrofished. Very few } \\
\text { RBT's present for large area }\end{array}$ \\
\hline \multirow[t]{3}{*}{ NF Coppei } & NFC-1* & $8 / 17$ & 30 & $\begin{array}{l}\text { Three } 1+\& \text { one } 206 \text { mm RBT; } \\
\text { RBT's - uncommon, crayfish - } \\
\text { rare; SCP - common }\end{array}$ & $\begin{array}{l}\text { Light effort with mainly } \\
\text { pools electrofished }\end{array}$ \\
\hline & $\mathrm{NFC}-2 *$ & $8 / 17$ & $.1 \mathrm{mi}$ & $\begin{array}{l}\text { Five 1+ RBT's; two age classes } \\
\text { observed; RBT's - uncommon, } \\
\text { dace, SCP - common }\end{array}$ & Moderate effort \\
\hline & $\mathrm{NFC}-4 *$ & $8 / 17$ & 5 & $\begin{array}{l}\text { Five } 1+\text { RBT's, dace-common, } \\
\text { SCP - abundant, crayfish - } \\
\text { uncommon }\end{array}$ & $\begin{array}{l}\text { Only one pool electrofished } \\
\text { to collect DNA samples }\end{array}$ \\
\hline NF Dry Ck & NFD-1 & $8 / 24$ & 58 & $\begin{array}{l}\text { Thirteen } 1+\& \text { one } 237 \mathrm{~mm} \\
\text { RBT, RBT's, dace - uncommon; } \\
\text { SCP - common. }\end{array}$ & $\begin{array}{l}\text { Moderately intensive survey } \\
\text { Sampled mainly pools. } \\
\text { Little water in stream }\end{array}$ \\
\hline Walla Walla & WW-3 & $8 / 1$ & 38 & $\begin{array}{l}\text { Six } 1+\& \text { one } 273 \mathrm{~mm} \text { hatchery } \\
\text { RBT; dace, RSS - abundant; } \\
\text { RSS - abundant; sucker, NPM, } \\
\text { SCP - common }\end{array}$ & $\begin{array}{l}\text { Light survey - area deep and } \\
\text { wide - difficult to survey }\end{array}$ \\
\hline
\end{tabular}


Relative abundance for qualitative electrofishing sites in the Walla Walla River basin, 2000.

\begin{tabular}{|c|c|c|c|c|c|}
\hline Stream & Site \# & Date & $\begin{array}{l}\text { Approx Site } \\
\text { Length (m) }\end{array}$ & Relative Abundance* & Comments \\
\hline $\begin{array}{l}\text { E ast Little } \\
\text { Walla Walla }\end{array}$ & ELW-4 & $8 / 1$ & 72 & $\begin{array}{l}\text { One 290mm RBT(H) \& one } \\
\text { 127mm MTW found; NPM, } \\
\text { 3-spine stickleback, SCP - } \\
\text { common; dace - uncommon; } \\
\text { crayfish; RBT - rare }\end{array}$ & Moderate survey intensity \\
\hline \multirow[t]{4}{*}{$\begin{array}{l}\text { West Little } \\
\text { Walla Walla }\end{array}$} & WLW-1 & $7 / 18$ & 90 & $\begin{array}{l}\text { No RBT's found; two age classes } \\
\text { of RSS, dace, SCP - common; } \\
\text { suckers - uncommon }\end{array}$ & $\begin{array}{l}\text { Intense electrofishing effort. } \\
\text { Good habitat, no RBT's }\end{array}$ \\
\hline & WLW-2 & $7 / 12$ & 45 & $\begin{array}{l}\text { Two 0+ RBT's found; RBT's - } \\
\text { rare; dace, crayfish - common; } \\
\text { RSS - uncommon }\end{array}$ & $\begin{array}{l}\text { Moderate survey intensity. } \\
\text { Very little water with dense } \\
\text { grass }\end{array}$ \\
\hline & WLW-3 & $7 / 18$ & 100 & No fish found & $\begin{array}{l}\text { Light survey targeting } \\
\text { mostly pools. Difficult to } \\
\text { survey due to dense grass } \\
\text { and shrubs }\end{array}$ \\
\hline & WLW-4 & $7 / 12$ & 200 & $\begin{array}{l}\text { One } 1+\text { RBT found; shiner - } \\
\text { common; dace - uncommon; } \\
\text { crayfish - rare }\end{array}$ & $\begin{array}{l}\text { Light effort targeting } \\
\text { mostly pools due to dense } \\
\text { grass and shrubs. Little } \\
\text { water }\end{array}$ \\
\hline \multirow[t]{5}{*}{ Mill Ck } & MC-1 & $8 / 23$ & 19 & $\begin{array}{l}\text { Twenty-nine } 1+(68-196 \mathrm{~mm}) \& \\
\text { Twenty-one }>8 \text { in }(200-345 \mathrm{~mm}) \\
\text { RBT's found; dace, RSS, RBT - } \\
\text { common; SCP, ameocete - } \\
\text { uncommon }\end{array}$ & $\begin{array}{l}\text { Moderate intensity } \\
\text { electrofish }\end{array}$ \\
\hline & MC-2 & $8 / 23$ & 38 & $\begin{array}{l}\text { Nine } 1+(66-196 \mathrm{~mm}) \& \text { three } \\
>8 \text { in }(220-395 \mathrm{~mm}) \text { RBT's; RSS, } \\
\text { RBT, dace, sucker - common; } \\
\text { SCP - uncommon }\end{array}$ & $\begin{array}{l}\text { Moderate electrofishing } \\
\text { effort targeting mostly } \\
\text { below weirs }\end{array}$ \\
\hline & $\mathrm{MC}-3$ & $8 / 23$ & 38 & $\begin{array}{l}\text { Three } 1+(74,86 \& 184 \mathrm{~mm}) \& \\
\text { five }>8 \text { in }(201-370 \mathrm{~mm}) \text { RBT's; } \\
\text { dace, SCP, crayfish, RSS, } \\
\text { sucker - common; ameocete, } \\
\text { RBT - uncommon }\end{array}$ & $\begin{array}{l}\text { Moderate effort. Surveyed } \\
\text { mostly pools }\end{array}$ \\
\hline & MC-4 & $7 / 5$ & 37 & $\begin{array}{l}\text { No RBT's found; SCP, dace, } \\
\text { RSS - common }\end{array}$ & $\begin{array}{l}\text { Light effort - sampled } \\
\text { mainly below weirs }\end{array}$ \\
\hline & MC-6 & $8 / 23$ & 145 & $\begin{array}{l}\text { No RBT's found; two age classes } \\
\text { sucker, dace - abundant; two age } \\
\text { classes RSS \& SCP - common }\end{array}$ & $\begin{array}{l}\text { Moderate electrofishing } \\
\text { effort }\end{array}$ \\
\hline
\end{tabular}


Relative abundance for qualitative electrofishing sites in the Walla Walla River basin, 2000.

\begin{tabular}{|c|c|c|c|c|c|}
\hline$\underline{\text { Stream }}$ & Site \# & Date & $\begin{array}{l}\text { Approx Site } \\
\text { Length (m) }\end{array}$ & Relative Abundance* & Comments \\
\hline \multirow[t]{11}{*}{ Mill Ck } & MC-7 & $7 / 5$ & 148 & $\begin{array}{l}\text { Two 0+ (60 \& 64mm), two } 1+ \\
(163 \& 176 \mathrm{~mm}) \& \text { four }>8 \text { in } \\
\text { (203 - 291mm) RBT's; dace, } \\
\text { SCP, RSS - abundant }\end{array}$ & $\begin{array}{l}\text { Moderate effort - pools } \\
\text { below weirs sampled. Few } \\
\text { RBT's found for large area }\end{array}$ \\
\hline & MC-8 & $8 / 23$ & 152 & $\begin{array}{l}\text { Nine } 1+(70-197 \mathrm{~mm}) \& \text { nine } \\
\text { > } 8 \text { in }(226 \text { - 396mm) RBT's; } \\
\text { dace, sucker - abundant; shiners, } \\
\text { SCP - common }\end{array}$ & Intense electrofish survey \\
\hline & MC-8 & $7 / 5$ & 161 & $\begin{array}{l}\text { Three } 1+(175-187 \mathrm{~mm}) \& \text { two } \\
>8 \text { in }(236 \& 299 \mathrm{~mm}) \mathrm{RBT} \\
\text { three age classes of dace, sucker, } \\
\text { RSS, SCP - abundant }\end{array}$ & $\begin{array}{l}\text { Moderate survey effort with } \\
\text { mostly pools below weirs } \\
\text { sampled }\end{array}$ \\
\hline & MC-9 & $7 / 5$ & 55.5 & $\begin{array}{l}\text { Two } 0+\& \text { two } 1+\text { RBT's; dace, } \\
\text { SCP, RSS - abundant }\end{array}$ & $\begin{array}{l}\text { Intense survey. Area is } \\
\text { more like wetlands }\end{array}$ \\
\hline & MC-11 & $8 / 10$ & 30 & $\begin{array}{l}\text { Nine RBT's ( } 80-115 \mathrm{~mm}) \text {; RBT, } \\
\text { sucker, dace, SCP - common; } \\
\text { crayfish - uncommon }\end{array}$ & $\begin{array}{l}\text { Light effort. Mainly } \\
\text { electrofished to collect } \\
\text { weights }\end{array}$ \\
\hline & MC-12 & $8 / 10$ & $2 \mathrm{mi}$. & $\begin{array}{l}168 \text { RBT's } 64-119 \mathrm{~mm} \text { found; } \\
\text { RBT, dace, RSS, BLS, crayfish, } \\
\text { SCP - common; MTW, long-nose } \\
\text { dace - rare }\end{array}$ & $\begin{array}{l}\text { Intensive electrofishing } \\
\text { effort }\end{array}$ \\
\hline & MC-14 & $7 / 6$ & 93.8 & $\begin{array}{l}\text { One } 63 \mathrm{~mm} \text { RBT found; RBT, } \\
\text { SCP - rare; two age classes of } \\
\text { RSS, sucker, NPM - common; } \\
\text { dace - abundant; }\end{array}$ & $\begin{array}{l}\text { Moderate effort. Rt bank } \\
\text { void of life due to Chlorine } \\
\text { leak. Most fish had bumps } \\
\text { and lesions. }\end{array}$ \\
\hline & MC-15 & $7 / 6$ & 89.8 & $\begin{array}{l}\text { Two RBT's (58 \& 255mm); } \\
\text { RSS, dace, NPM - abundant }\end{array}$ & $\begin{array}{l}\text { Moderate effort. Strong } \\
\text { chlorine smell in river }\end{array}$ \\
\hline & MC-16 & $8 / 22$ & 50 & No fish found & $\begin{array}{l}\text { Moderate effort. Very strong } \\
\text { chlorine smell in river }\end{array}$ \\
\hline & MC-17 & $8 / 22$ & 189 & $\begin{array}{l}\text { Ten } 1+\text { and two }>8 \text { in RBT's } \\
\text { (223 \& } 363 \text { (hatchery) mm); } \\
\text { RBT's - rare; dace - abundant; } \\
\text { BLS, RSS - common, SCP - } \\
\text { uncommon }\end{array}$ & $\begin{array}{l}\text { Intense effort. Good } \\
\text { habitat, but chlorine smell } \\
\text { smell in river }\end{array}$ \\
\hline & MC-18 & $7 / 6$ & 76.5 & $\begin{array}{l}\text { Ten } 0+\text {, one } 1+, \& \text { one } 310 \mathrm{~mm} \\
\text { hatchery RBT found; dace, SCP } \\
\text { RSS - abundant; NPM, sucker - } \\
\text { common }\end{array}$ & Moderate survey intensity \\
\hline
\end{tabular}


Relative abundance for qualitative electrofishing sites in the Walla Walla River basin, 2000.

\begin{tabular}{|c|c|c|c|c|c|}
\hline Stream & Site \# & Date & $\begin{array}{l}\text { Approx Site } \\
\text { Length (m) }\end{array}$ & Relative Abundance* & Comments \\
\hline S Russell & SRC-1 & $7 / 11$ & 33 & No fish found. & Intensive survey \\
\hline \multirow[t]{7}{*}{ Russell } & $\mathrm{RC}-1$ & $7 / 11$ & 50 & No fish found. & Intensive survey \\
\hline & $\mathrm{RC}-2$ & $7 / 12$ & 23 & No fish found. & Intensive survey \\
\hline & $\mathrm{RC}-3$ & $7 / 11$ & 125 & $\begin{array}{l}\text { One } 190 \mathrm{~mm} \text { RBT found. No } \\
\text { other fish found. }\end{array}$ & Intensive survey \\
\hline & $\mathrm{RC}-4$ & $7 / 11$ & 80 & $\begin{array}{l}\text { 3-4 age classes of RBT. } \\
\text { Seventeen RBT’s ranging from } \\
63-252 \mathrm{~mm} \text {. Dace - common, } \\
\text { SCP \& RSSuncommon. }\end{array}$ & Intensive survey \\
\hline & $\mathrm{RC}-5$ & $7 / 11$ & 64 & $\begin{array}{l}\text { Seven RBT representing } \\
2 \text { age classes found. Dace - } \\
\text { common, SCP- uncommon } \\
\text { Lamprey - rare }\end{array}$ & Intensive survey \\
\hline & RC-6 & $7 / 12$ & 71 & $\begin{array}{l}\text { Two RBT found } 166 \mathrm{~mm} \& \\
182 \mathrm{~mm} \text {. SCP - common }\end{array}$ & Intensive survey \\
\hline & $\mathrm{RC}-7$ & $7 / 12$ & 58 & $\begin{array}{l}\text { Two 0+ RBT found. Dace \& } \\
\text { RSS abundant. SCP - common } \\
\text { Suckers - uncommon }\end{array}$ & Moderate \\
\hline \multirow[t]{5}{*}{ Garrison } & GC-2 & $7 / 20$ & 38 & $\begin{array}{l}\text { No RBT's found; RSS, dace, } \\
\text { ameocete - common; NPM - } \\
\text { rare; SCP - abundant }\end{array}$ & Light survey. Poor habitat \\
\hline & GC-3 & $7 / 20$ & 37.7 & $\begin{array}{l}\text { No RBT's found; crayfish \& } \\
\text { SCP - common }\end{array}$ & $\begin{array}{l}\text { Moderate survey intensity } \\
\text { with mostly pools } \\
\text { electrofished }\end{array}$ \\
\hline & GC-4 & $7 / 20$ & 37 & $\begin{array}{l}\text { No RBT's found; SCP - } \\
\text { abundant; RSS - rare }\end{array}$ & $\begin{array}{l}\text { Moderate survewy intensity } \\
\text { with mostly pools } \\
\text { electrofished }\end{array}$ \\
\hline & GC-5 & $7 / 20$ & 85.5 & $\begin{array}{l}\text { No RBT's found; NPM, SCP, } \\
\text { sucker, dace - common; RSS - } \\
\text { Abundant }\end{array}$ & Intense survey \\
\hline & GC-7 & $7 / 18$ & $.2 \mathrm{mi}$ & $\begin{array}{l}\text { No RBT's found; two age classes } \\
\text { dace - common }\end{array}$ & $\begin{array}{l}\text { Moderate survey intensity. } \\
\text { Difficult to survey due to } \\
\text { dense vegetation growth }\end{array}$ \\
\hline
\end{tabular}


Relative abundance for qualitative electrofishing sites in the Walla Walla River basin, 2000.

\begin{tabular}{|c|c|c|c|c|c|}
\hline Stream & Site \# & Date & $\begin{array}{l}\text { Approx Site } \\
\text { Length (m) }\end{array}$ & Relative Abundance* & Comments \\
\hline Caldwell & $\mathrm{CD}-2$ & $7 / 11$ & 56 & $\begin{array}{l}\text { No RBT's found; one age class } \\
\text { of dace - common }\end{array}$ & $\begin{array}{l}\text { Moderate survey effort. } \\
\text { Poor habitat - very muddy }\end{array}$ \\
\hline \multirow[t]{2}{*}{ Mud Ck } & MC-1 & $8 / 16$ & 20 & $\begin{array}{l}\text { No RBT's found; dace, 3-spine } \\
\text { stickleback - abundant; SCP - } \\
\text { common; crayfish - rare }\end{array}$ & $\begin{array}{l}\text { Moderate intensity survey. } \\
\text { Poor habitat with heavy } \\
\text { cattle use, mud botton and no } \\
\text { riparian }\end{array}$ \\
\hline & MC-2 & $8 / 16$ & 100 & $\begin{array}{l}\text { No RBT's found; 3-spine } \\
\text { stickleback - common; dace - } \\
\text { uncommon }\end{array}$ & $\begin{array}{l}\text { Light effort. Deep, slow } \\
\text { pools with no riparian. } \\
\text { Heavy cattle use }\end{array}$ \\
\hline Pine $\mathrm{Ck}$ & PC-1 & $8 / 28$ & 140 & $\begin{array}{l}\text { No RBT's found; dace - rare; } \\
\text { small mouth bass - uncommon; } \\
\text { madtom - rare }\end{array}$ & $\begin{array}{l}\text { Light survey intensity. Poor } \\
\text { habitat with deep pools and } \\
\text { no flow. Thick scum layer } \\
\text { on surface. }\end{array}$ \\
\hline \multirow[t]{4}{*}{ Stone Ck } & $\mathrm{SC}-1$ & $8 / 22$ & 30 & $\begin{array}{l}\text { No RBT's found; dace, SCP, } \\
\text { crayfish - common; ameocetes - } \\
\text { rare }\end{array}$ & $\begin{array}{l}\text { Moderate survey intensity. } \\
\text { Good riparian, but little flow } \\
\text { and mud substrate }\end{array}$ \\
\hline & $\mathrm{SC}-2$ & $7 / 20$ & 44.1 & $\begin{array}{l}\text { No RBT's found; crayfish, } \\
\text { SCP - uncommon; dace - rare }\end{array}$ & $\begin{array}{l}\text { Light survey intensity. Very } \\
\text { dense grass and cattails. } \\
\text { Very little water. Difficult } \\
\text { to electrofish. }\end{array}$ \\
\hline & $\mathrm{SC}-3$ & $7 / 21$ & 200 & $\begin{array}{l}\text { No RBT's found; two age } \\
\text { classes of dace, SCP - abundant; } \\
\text { ameocete, crayfish - common; } \\
\text { sucker, NPM - rare }\end{array}$ & $\begin{array}{l}\text { Intense survey. Wetland } \\
\text { like habitat with little open } \\
\text { water, dense grass and mud } \\
\text { bottom }\end{array}$ \\
\hline & $\mathrm{SC}-4$ & $7 / 20$ & 24 & $\begin{array}{l}\text { No RBT's found; dace - } \\
\text { abundant }\end{array}$ & $\begin{array}{l}\text { Light survey intensity. } \\
\text { Very poor habitat with little } \\
\text { flow and mud bottom. Poor } \\
\text { ripairian }\end{array}$ \\
\hline Doan Ck & $\mathrm{DN}-2$ & $8 / 15$ & 30 & $\begin{array}{l}\text { No RBT's found; SCP, dace - } \\
\text { common }\end{array}$ & $\begin{array}{l}\text { Light survey intensity. } \\
\text { Dense grass growth }\end{array}$ \\
\hline \multirow[t]{2}{*}{ Cold Ck } & $\mathrm{CC}-1$ & $8 / 22$ & 30 & $\begin{array}{l}\text { No RBT's found; three age } \\
\text { classes of dace - common; } \\
\text { two age classes of SCP - } \\
\text { common. }\end{array}$ & $\begin{array}{l}\text { Light survey intensity. } \\
\text { Good riparian, but no bank } \\
\text { stability and mud bottom }\end{array}$ \\
\hline & $\mathrm{CC}-2$ & $8 / 16$ & 30 & $\begin{array}{l}\text { No RBT's found; two age classes } \\
\text { of dace and SCP; dace - abundant } \\
\text { SCP - common }\end{array}$ & $\begin{array}{l}\text { Moderate survey intensity. } \\
\text { Very poor habitat with } \\
\text { heavy cattle use, no riparian \& } \\
\text { mud substrate }\end{array}$ \\
\hline
\end{tabular}


Relative abundance for qualitative electrofishing sites in the Walla Walla River basin, 2000.

\begin{tabular}{|c|c|c|c|c|c|}
\hline$\underline{\text { Stream }}$ & Site \# & Date & $\begin{array}{l}\text { Approx Site } \\
\text { Length (m) }\end{array}$ & Relative Abundance* & Comments \\
\hline \multirow[t]{2}{*}{ Cottonwood } & CCC-4 & $7 / 6$ & 117 & No fish found & $\begin{array}{l}\text { Light survey. Little water } \\
\text { with many dry spots. No } \\
\text { riparian and very little flow }\end{array}$ \\
\hline & CCC-5 & $7 / 5$ & 30.5 & $\begin{array}{l}\text { Fifteen } 0+(56-78 \mathrm{~mm}) \text {, seven } \\
1+(133-153 \mathrm{~mm}), \text { one } 221 \mathrm{~mm} \\
\text { RBT's found; dace - uncommon; } \\
\text { SCP - rare }\end{array}$ & $\begin{array}{l}\text { Moderate intensity survey. } \\
\text { Mainly pools sampled }\end{array}$ \\
\hline Whitney & WH-1 & $8 / 2$ & $3.5 \mathrm{mi}$ & $\begin{array}{l}\text { RBT's - common; T-frog - } \\
\text { common }\end{array}$ & $\begin{array}{l}\text { Moderate intense survey. } \\
\text { Some larger RBT's to 12in } \\
\text { found. }\end{array}$ \\
\hline \multirow[t]{3}{*}{ Corral Ck } & $\mathrm{CC}-1$ & $7 / 31$ & 200 & $\begin{array}{l}\text { Four BT (146-168mm) found; } \\
\text { Tailed frog - rare }\end{array}$ & $\begin{array}{l}\text { Light electrofish survey with } \\
\text { mostly pools sampled }\end{array}$ \\
\hline & $\mathrm{CC}-2$ & $7 / 31$ & 200 & $\begin{array}{l}\text { No fish found; Tailed frog - } \\
\text { rare }\end{array}$ & $\begin{array}{l}\text { Light electrofish survey with } \\
\text { mostly pools sampled }\end{array}$ \\
\hline & $\mathrm{CC}-3$ & $7 / 31$ & 40 & $\begin{array}{l}\text { Five BT }(121-157 \mathrm{~mm}) \text { found; } \\
\text { No RBT's found }\end{array}$ & Intense electrofish survey. \\
\hline $\begin{array}{l}\text { Mustard } \\
\text { Hollow }\end{array}$ & MH-1 & $8 / 21$ & 10 & $\begin{array}{l}\text { No RBT's found; one red-eared } \\
\text { sunfish captured }\end{array}$ & $\begin{array}{l}\text { Light electrofish survey. } \\
\text { Very poor habitat with dense } \\
\text { grass and mud substrate. }\end{array}$ \\
\hline $\begin{array}{l}\text { Cougar } \\
\text { Hollow } \\
\end{array}$ & $\mathrm{CH}-1$ & $8 / 21$ & 100 & No water & \\
\hline $\begin{array}{l}\text { Johnson } \\
\text { Hollow } \\
\end{array}$ & JH-1 & $8 / 21$ & 100 & No fish found & Almost no water \\
\hline $\begin{array}{l}\text { South Fk } \\
\text { Patit }\end{array}$ & SCP-3 & $8 / 21$ & 100 & $\begin{array}{l}\text { Three age classes of RBT's } \\
\text { found; RBT's abundant; } \\
\text { SCP - rare }\end{array}$ & $\begin{array}{l}\text { Moderate electrofish effort } \\
\text { sampling mostly pools }\end{array}$ \\
\hline $\begin{array}{l}\text { Patrick Sp } \\
\text { Creek }\end{array}$ & PSC-1 & $7 / 18$ & 30 & $\begin{array}{l}\text { One } 126 \mathrm{~mm} \text { RBT \& two BT } \\
\text { (93 \& 84mm); BT, RBT \& } \\
\text { tailed frog - rare }\end{array}$ & $\begin{array}{l}\text { Light electrofish effort. } \\
\text { Likely barrier in stream } \\
\text { with fish found below falls, } \\
\text { No fish found above }\end{array}$ \\
\hline Hatley Gulch & HG-1 & $8 / 21$ & 15 & $\begin{array}{l}\text { No fish found } \\
\text { Very little water. }\end{array}$ & Moderate electrofish effort. \\
\hline
\end{tabular}


Relative abundance for qualitative electrofishing sites in the Walla Walla River basin, 2000.

\begin{tabular}{|c|c|c|c|c|c|}
\hline Stream & Site \# & Date & $\begin{array}{l}\text { Approx Site } \\
\text { Length (m) }\end{array}$ & Relative Abundance* & Comments \\
\hline Davis Hollow & DH-1 & $8 / 21$ & & No water & \\
\hline \multirow[t]{2}{*}{ Titus Ck } & TC-1 & $9 / 11$ & 25 & $\begin{array}{l}\text { One } 281 \mathrm{~mm} \text { RBT found; RSS, } \\
\text { SCP, dace - common; BLS - } \\
\text { uncommon }\end{array}$ & $\begin{array}{l}\text { Light survey intensity due } \\
\text { to dense and shrub growth. } \\
\text { Difficult access }\end{array}$ \\
\hline & TC-3 & $9 / 11$ & 30 & $\begin{array}{l}\text { Three } 1+(120-143 \mathrm{~mm}) \& \\
\text { two > } 8 \text { in }(234 \& 243 \mathrm{~mm}) \\
\text { RBT's; SCP, BLS - uncommon; } \\
\text { dace - common; RSS - abundant }\end{array}$ & $\begin{array}{l}\text { Moderate intensity survey. } \\
\text { Very deep and muddy pools } \\
\text { with little flow. Difficult to } \\
\text { sample }\end{array}$ \\
\hline
\end{tabular}

Bryant Ck $\quad$ BC-1 $\quad 8 / 22 \quad 30$

One $97 \mathrm{~mm}$ RBT found; dace,

SCP - common

Intense electrofish survey.

grass and mud substrate

\begin{tabular}{|c|c|c|c|c|c|}
\hline \multirow[t]{2}{*}{ Burnt Fk } & $\mathrm{BF}-1^{*}$ & $8 / 7$ & 60 & $\begin{array}{l}\text { Six 0+ (58-64mm) \& twenty- } \\
\text { three } 1+(73-175 \mathrm{~mm}) \text { RBT's } \\
\text { found; twelve BT }(102-273 \mathrm{~mm}) \text {; } \\
\text { Tailed frog - uncommon }\end{array}$ & $\begin{array}{l}\text { Intense electrofish survey. } \\
\text { Excellent habitat }\end{array}$ \\
\hline & $\mathrm{BF}-2$ & $8 / 7$ & 30 & $\begin{array}{l}\text { Two 0+ }(62 \& 70) \text {, fourteen } \\
1+(99-192 \mathrm{~mm}) \& \text { one } 204 \mathrm{~mm} \\
\text { RBT found; RBT's - common; } \\
\text { tailed frog - rare; SCP - } \\
\text { uncommon }\end{array}$ & $\begin{array}{l}\text { Intense electrofish survey. } \\
\text { Excellent habitat }\end{array}$ \\
\hline \multirow[t]{5}{*}{ Robinson Fk } & $\mathrm{RF}-1 *$ & $8 / 15$ & 30 & $\begin{array}{l}\text { Five RBT's }(81-110 \mathrm{~mm}) \\
\text { sampled for DNA; RBT, SCP - } \\
\text { common }\end{array}$ & $\begin{array}{l}\text { Light effort. Little water } \\
\text { with small pools. Lots of } \\
\text { algae on rocks }\end{array}$ \\
\hline & $\mathrm{RF}-2 *$ & $8 / 15$ & 30 & $\begin{array}{l}\text { Five RBT's (94-128mm) } \\
\text { sampled for DNA; RBT, SCP - } \\
\text { common }\end{array}$ & $\begin{array}{l}\text { Light effort. Good habitat } \\
\text { with some large pools }\end{array}$ \\
\hline & $\mathrm{RF}-3^{*}$ & $8 / 15$ & 5 & $\begin{array}{l}\text { Five RBT's (56-119mm) } \\
\text { sampled for DNA; RBT, SCP - } \\
\text { common }\end{array}$ & $\begin{array}{l}\text { Light effort. Electrofished } \\
\text { two pools }\end{array}$ \\
\hline & RF-4 & $8 / 15$ & 16 & $\begin{array}{l}\text { Five RBT's (85-138mm) } \\
\text { sampled for DNA; RBT, SCP - } \\
\text { common; crayfish - uncommon }\end{array}$ & $\begin{array}{l}\text { Light survey. Surveyed } \\
\text { mostly pools. Good habitat }\end{array}$ \\
\hline & $\mathrm{RF}-5^{*}$ & $8 / 15$ & 12 & $\begin{array}{l}\text { Five RBT's (92-116mm) } \\
\text { sampled for DNA; RBT, SCP, }\end{array}$ & $\begin{array}{l}\text { Light survey. Sampled } \\
\text { mostly pools. Site exposed }\end{array}$ \\
\hline
\end{tabular}


Relative abundance for qualitative electrofishing sites in the Walla Walla River basin, 2000.

\begin{tabular}{|c|c|c|c|c|c|}
\hline Stream & Site \# & Date & $\begin{array}{l}\text { Approx Site } \\
\text { Length (m) }\end{array}$ & Relative Abundance* & Comments \\
\hline \multirow[t]{11}{*}{ Robinson Fk } & & & & crayfish - common & with no shade and no LOD \\
\hline & RF-6* & $8 / 15$ & 17 & $\begin{array}{l}\text { Five RBT's (83-143mm) } \\
\text { sampled for DNA; RBT, SCP, } \\
\text { crayfish - common }\end{array}$ & $\begin{array}{l}\text { Light survey. Sampled } \\
\text { mostly pools. Little shade } \\
\text { and poor riparian. }\end{array}$ \\
\hline & $\mathrm{RF}-7^{*}$ & $8 / 15$ & 18 & $\begin{array}{l}\text { Five RBT's (84-121mm) } \\
\text { sampled for DNA; RBT, SCP, } \\
\text { crayfish - common }\end{array}$ & $\begin{array}{l}\text { Light survey. Sampled } \\
\text { mostly pools }\end{array}$ \\
\hline & RF-8* & $8 / 15$ & 3 & $\begin{array}{l}\text { Five RBT's (103-127mm) } \\
\text { sampled for DNA }\end{array}$ & $\begin{array}{l}\text { Very light survey. Sampled } \\
\text { only one pool. }\end{array}$ \\
\hline & RF-8* & $8 / 15$ & 150 & $\begin{array}{l}\text { Nine RBT's (76-162mm) found; } \\
\text { RBT's - uncommon }\end{array}$ & Moderate intensity survey. \\
\hline & RF-9* & $8 / 15$ & 9 & $\begin{array}{l}\text { Five RBT's }(94-127 \mathrm{~mm}) \\
\text { sampled for DNA }\end{array}$ & $\begin{array}{l}\text { Very light survey. Only } \\
\text { sampled one pool. }\end{array}$ \\
\hline & $\mathrm{RF}-10^{*}$ & $8 / 15$ & 26 & $\begin{array}{l}\text { Five RBT's ( } 88-118 \mathrm{~mm}) \\
\text { sampled for DNA; RBT, SCP, } \\
\text { crayfish - common }\end{array}$ & $\begin{array}{l}\text { Light survey. Sampled } \\
\text { mostly pools. }\end{array}$ \\
\hline & RF-13* & $8 / 15$ & 13 & $\begin{array}{l}\text { Five RBT's (105-141mm) } \\
\text { sampled for DNA; RBT, SCP, } \\
\text { dace \& crayfish - common }\end{array}$ & $\begin{array}{l}\text { Very light survey. Sampled } \\
\text { two pools. Poor riparian. }\end{array}$ \\
\hline & $\mathrm{RF}-14^{*}$ & $8 / 15$ & 65 & $\begin{array}{l}\text { Five RBT's (91-117mm) } \\
\text { sampled for DNA; RBT, SCP, } \\
\text { crayfish - common }\end{array}$ & $\begin{array}{l}\text { Light survey. Sampled } \\
\text { mostly pools. Mostly larger } \\
\text { 1+ RBT's found }\end{array}$ \\
\hline & Pond trib & $7 / 19$ & 600 & $\begin{array}{l}\text { Nine RBT's ( } 30-225 \mathrm{~mm}) \text { found; } \\
\text { no other fish found; RBT's - } \\
\text { rare }\end{array}$ & $\begin{array}{l}\text { Intense electrofish survey. } \\
\text { Little water and heavy cattle } \\
\text { use, but good riparian }\end{array}$ \\
\hline & $\begin{array}{l}\text { Headwaters } \\
\text { down }\end{array}$ & $7 / 19$ & $\sim 3 \mathrm{mi}$ & $\begin{array}{l}\text { No RBT's found } 1^{\text {st }} \text { two miles; } \\
\text { tailed frogs - common; RBT's - } \\
\text { uncommon last mile; tailed } \\
\text { frogs- uncommon }\end{array}$ & $\begin{array}{l}\text { Moderate intensity survey. } \\
\text { Steep gradient and multiple } \\
\text { barriers on upper reach. } \\
\text { Lower reach has good habitat } \\
\text { with good riparian. }\end{array}$ \\
\hline
\end{tabular}




\begin{tabular}{|c|c|c|c|c|c|c|c|c|c|c|c|c|c|c|c|}
\hline \multicolumn{4}{|l|}{ Appendix F. Table 1.} & \multicolumn{9}{|c|}{ Relative Abundance - Non- Salmonids 2000} & \multicolumn{3}{|c|}{ Touchet River } \\
\hline Species & 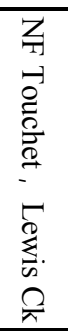 & 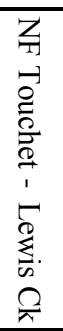 & 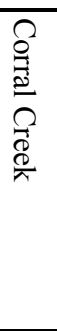 & 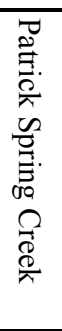 & 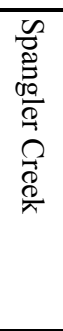 & 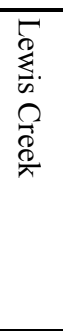 & 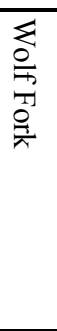 & 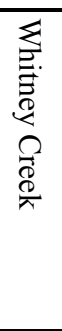 & 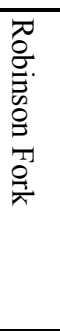 & 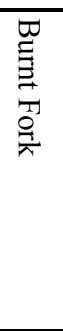 & 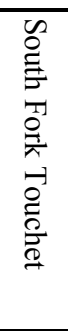 & $\begin{array}{l}\overrightarrow{+} \\
\stackrel{0}{0} \\
\stackrel{0}{\sigma} \\
\overrightarrow{0} \\
\stackrel{0}{9}\end{array}$ & 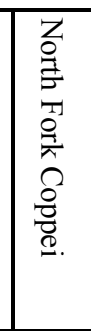 & 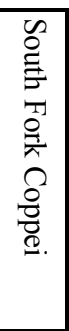 & $\begin{array}{l}\Omega \\
\frac{0}{2} \\
\mathbb{Q} \\
\Omega \\
\vec{D} \\
\frac{\mathbb{D}}{\pi}\end{array}$ \\
\hline $\begin{array}{r}\text { Petromyzontide } \\
\text { Lamprey }\end{array}$ & 1 & 1 & 0 & 0 & 0 & 0 & $\mathrm{P}$ & 0 & $\mathrm{P}$ & 0 & 1 & 1 & 1 & 1 & $\mathrm{P}$ \\
\hline $\begin{array}{r}\text { Cyprinidae } \\
\text { Speckled dace } \\
\text { Rhinichtys osculus }\end{array}$ & 4 & 3 & 0 & 0 & 0 & 0 & 1 & 0 & 2 & 0 & 4 & 4 & 3 & 3 & 4 \\
\hline $\begin{array}{r}\text { Chiselmouth } \\
\text { Acroheilus alutaceus }\end{array}$ & 0 & 0 & 0 & 0 & 0 & 0 & 0 & 0 & 0 & 0 & 0 & 0 & 0 & 0 & 0 \\
\hline $\begin{array}{r}\text { Redside shiner } \\
\text { Richardsonius balteatus }\end{array}$ & 0 & 1 & 0 & 0 & 0 & 0 & 0 & 0 & 0 & 0 & 2 & 2 & 0 & 0 & 2 \\
\hline $\begin{array}{l}\text { Northern pikeminnow } \\
\text { Ptychocheilus oregonesis }\end{array}$ & 0 & 0 & 0 & 0 & 0 & 0 & 0 & 0 & 0 & 0 & 0 & 0 & 0 & 0 & 1 \\
\hline $\begin{array}{r}\text { Catostomidae } \\
\text { Suckers }^{\mathrm{a}} \\
\text { Catostomus sp. }\end{array}$ & 0 & 0 & 0 & 0 & 0 & 0 & 0 & 0 & 0 & 0 & $\mathrm{P}$ & $\mathrm{P}$ & $\mathrm{P}$ & $\mathrm{P}$ & $\mathrm{P}$ \\
\hline $\begin{array}{r}\text { Gasterosteidae } \\
\text { Threespine stickleback } \\
\text { Gasterosteus aculeatus }\end{array}$ & 0 & 0 & 0 & 0 & 0 & 0 & 0 & 0 & 0 & 0 & 0 & 0 & 0 & 0 & 0 \\
\hline $\begin{array}{r}\text { Cottidae } \\
\text { Piute sculpin } \\
\text { Cottus beldingi }\end{array}$ & 3 & 3 & 0 & 0 & $\mathrm{P}$ & 1 & 3 & $\mathrm{P}$ & 3 & 1 & 3 & 3 & 2 & 3 & 0 \\
\hline $\begin{array}{l}\text { Margin sculpin } \\
\text { Cottus marginatus }\end{array}$ & 2 & 3 & 0 & 0 & $\mathrm{P}$ & 1 & 2 & 0 & 2 & 1 & 3 & 3 & 2 & 3 & 3 \\
\hline $\begin{array}{l}\text { Torrent sculpin } \\
\text { Cottus rhotheus }\end{array}$ & 0 & 0 & 0 & 0 & 0 & 0 & 0 & 0 & 0 & 0 & 0 & 2 & 0 & 0 & 0 \\
\hline $\begin{array}{l}\text { Tailed Frogs } \\
\text { Ascaphus truei }\end{array}$ & $3^{c}$ & 0 & $1^{\mathrm{b}}$ & $1^{\mathrm{b}}$ & 3 & 1 & $\mathrm{P}$ & $3^{b}$ & 1 & 1 & 0 & 0 & 0 & 0 & 0 \\
\hline $\begin{array}{r}\text { Crayfish } \\
\text { Pacifastacus Spp. }\end{array}$ & 2 & 3 & 0 & 0 & 0 & $\mathrm{P}$ & $\mathrm{P}$ & 0 & 3 & 0 & 2 & 3 & 1 & 1 & 3 \\
\hline
\end{tabular}




\begin{tabular}{|c|c|c|c|c|c|c|c|c|c|c|c|c|c|c|c|c|c|c|}
\hline Appendix F. Table 1. & \multicolumn{18}{|c|}{ Walla Walla River Basin } \\
\hline Species & 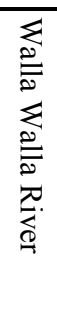 & 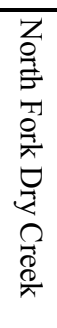 & 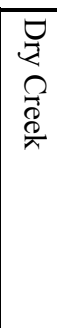 & 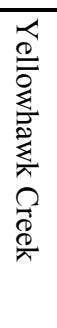 & 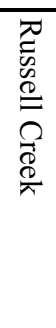 & 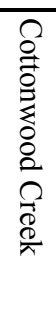 & 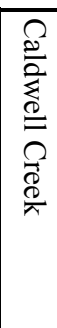 & 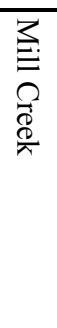 & 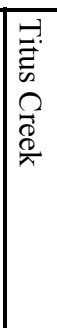 & 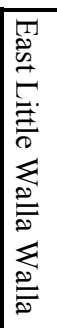 & 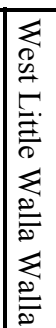 & 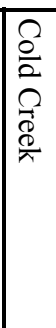 & 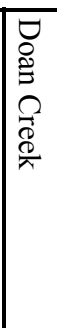 & 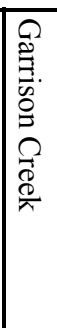 & 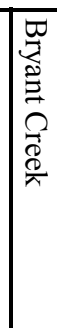 & 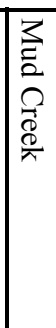 & 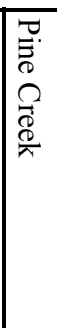 & 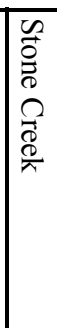 \\
\hline $\begin{array}{r}\text { Petromyzontide } \\
\text { Lamprey larvae }\end{array}$ & 1 & 0 & 1 & 1 & $P$ & 0 & 1 & 1 & 0 & 1 & $P$ & 0 & 0 & 1 & 0 & $P$ & $P$ & $1^{\mathrm{b}}$ \\
\hline $\begin{array}{r}\text { Cyprinidae } \\
\text { Speckled dace } \\
\text { Rhinichtys osculus }\end{array}$ & 4 & 1 & 3 & 3 & 4 & 3 & 3 & 4 & 3 & 3 & 3 & 4 & 3 & 3 & $3 b$ & $3^{\mathrm{b}}$ & $1^{\mathrm{b}}$ & $3^{\mathrm{b}}$ \\
\hline $\begin{array}{r}\text { Longnose dace } \\
\text { Rhinichtys cataractae }\end{array}$ & $\overline{0}$ & 0 & 0 & 0 & 0 & 0 & 0 & $\bar{P}$ & 0 & 0 & 0 & 0 & 0 & 0 & 0 & 0 & 0 & 0 \\
\hline $\begin{array}{r}\text { Chiselmouth } \\
\text { Acroheilus alutaceus }\end{array}$ & 3 & 0 & 0 & 0 & 0 & 0 & 0 & 1 & 0 & 0 & 0 & 0 & 0 & 0 & 0 & 0 & 0 & 0 \\
\hline $\begin{array}{r}\text { Redside shiner } \\
\text { Richardsonius balteatus } \\
\end{array}$ & 4 & 0 & 1 & 3 & 3 & 0 & 0 & 3 & 3 & 2 & 3 & 0 & 0 & 3 & 0 & 0 & 0 & 0 \\
\hline $\begin{array}{r}\text { Northern pikeminnow } \\
\text { Ptychocheilus } \\
\text { oregonesis }\end{array}$ & 3 & 0 & 0 & 1 & 0 & 0 & 0 & 2 & 0 & 2 & 0 & 0 & 0 & 0 & 0 & 0 & 0 & $1^{\mathrm{b}}$ \\
\hline $\begin{array}{r}\text { Ictaluridae } \\
\text { Tadpole Madtom } \\
\text { Notorus gyrinus }\end{array}$ & 0 & 0 & 0 & 0 & 0 & 0 & 0 & 0 & 0 & 0 & 0 & 0 & 0 & 0 & 0 & 0 & $1^{\mathrm{b}}$ & 0 \\
\hline $\begin{array}{r}\text { Catostomidae } \\
\text { Suckers } \\
\text { Catostomus sp. }\end{array}$ & 3 & 0 & $\mathrm{P}$ & 2 & 1 & $\mathrm{P}$ & 0 & 3 & 2 & 2 & 2 & 0 & 0 & 1 & 0 & 0 & 0 & $1^{\mathrm{b}}$ \\
\hline $\begin{array}{l}\text { Gasterosteidae } \\
\text { Threespine stickleback } \\
\text { Gasterosteus aculeatus }\end{array}$ & $\mathrm{P}$ & 0 & 0 & 0 & 0 & 0 & 0 & 0 & 0 & $\mathrm{P}$ & 0 & 0 & 0 & 0 & 0 & $4^{b}$ & 0 & 0 \\
\hline $\begin{array}{r}\text { Centrarchidae } \\
\text { Smallmouth Bass } \\
\text { Micropterus dolomieu }\end{array}$ & $\mathrm{P}$ & 0 & 0 & 0 & 0 & 0 & 0 & 0 & 0 & 0 & 0 & 0 & 0 & 0 & 0 & 0 & $2^{\mathrm{b}}$ & 0 \\
\hline $\begin{array}{r}\text { Cottidae } \\
\text { Piute sculpin } \\
\text { Cottus beldingi }\end{array}$ & 2 & 0 & 0 & 1 & 2 & 1 & 0 & 1 & 0 & 0 & 0 & 0 & 0 & 0 & 0 & 0 & 0 & 0 \\
\hline $\begin{array}{r}\text { Margin sculpin } \\
\text { Cottus marginatus } \\
\end{array}$ & 3 & 2 & 3 & 3 & 0 & 2 & 0 & 3 & 1 & 2 & 2 & 3 & 3 & 3 & $3^{b}$ & $2^{\mathrm{b}}$ & 0 & $3^{\mathrm{b}}$ \\
\hline $\begin{array}{l}\text { Torrent sculpin } \\
\text { Cottus rhotheus }\end{array}$ & 3 & 0 & 0 & 1 & 0 & 0 & 0 & $\mathrm{P}$ & 1 & 0 & 0 & 0 & 0 & 0 & 0 & 0 & 0 & 0 \\
\hline $\begin{array}{r}\text { Crayfish } \\
\text { Pacifastacus Spp. }\end{array}$ & 3 & 2 & 2 & 2 & 0 & $\mathrm{P}$ & 0 & 1 & 0 & 2 & 1 & 0 & $\mathrm{P}$ & $P$ & 0 & $\mathrm{P}$ & 0 & $3^{\mathrm{b}}$ \\
\hline
\end{tabular}

Table 2. Categories of relative abundance.

\begin{tabular}{lrr}
\hline Category & Count & Ranking Value \\
\hline Absent & 0 & 0 \\
Rare & $1-3$ & 1 \\
Uncommon & $4-10$ & 2 \\
Common & $11-100$ & 3 \\
Abundant & $100+$ & 4 \\
\hline
\end{tabular}

$\mathrm{P}=$ present,

a. Nnoted by genus only, not identified by species.

${ }^{\mathrm{b}}$. Relative abundance derive from qualitative electrofishing. 


\begin{tabular}{c}
\hline Appendix G. Preliminary Results of Instream Flow \\
Incremental Methodology for the Walla Walla River and \\
Mill Creek 1999
\end{tabular}




\section{Preliminary Results}

April 2, 2001
TO:
Glen Mendel
FROM:
Hal Beecher

SUBJECT: $\quad$ Instream flow study of the Walla Walla River and lower Mill Creek

The purpose of the study was to determine how summer flows in the Walla Walla River immediately downstream from the mouth of Mill Creek and in lower Mill Creek affect habitat conditions for juvenile steelhead. We used Physical Habitat Simulation (PHABSIM) models of the two study reaches to determine the distribution of water depths and velocities in relation to substrate and cover and to evaluate these distributions in relation to habitat selection preferences of juvenile steelhead.

The PHABSIM model does not address water temperature nor other aspects of water quality. It assumes a constant channel or at least one that is in dynamic equilibrium so that the frequency of mesohabitat types and microhabitat types will be represented by the model even if there are channel changes. The PHABSIM model does not address the following, which are important factors in maintaining or restoring watershed health:

-channel maintenance flows, including flushing flows

-flows to maintain riparian vegetation

-flows for outmigration of smolts.

\section{Site Selection}

We relied heavily on the judgement and experience of WDFW fish biologists (Glen Mendel and staff) who have worked in the Walla Walla River basin and know fish distribution and stream habitat characteristics throughout the basin. In October 1998, Glen Mendel and I flew the Walla Walla River from the Oregon border to the confluence with the Columbia River. We delineated reaches on a topographic map. Reaches were similar throughout in gradient, flow, and sinuosity. Based on the judgement of Glen Mendel, his staff, and my impressions, the reach selected was "typical" of a portion of the Walla Walla River considered important for steelhead because a few juveniles currently rear in this reach.

\section{Hydraulic Model Calibration}

We measured depths, velocities, and recorded substrate and cover at points along transects at our two sites in June and July, 1999 (Table 1). All measurements are in English units: flow is in cubic feet per second (cfs), depth in feet, and velocity in feet per second (fps).

\begin{tabular}{|l|l|l|}
\hline $\begin{array}{l}\text { Table 1. Transects and measured } \\
\text { flows in Walla Walla River and Mill } \\
\text { Creek }\end{array}$ & $\begin{array}{l}\text { Walla Walla River immediately } \\
\text { downstream from mouth of Mill Creek }\end{array}$ & $\begin{array}{l}\text { Mill Creek immediately upstream of } \\
\text { Wallula Road bridge }\end{array}$ \\
\hline number of transects & 9 & 8 \\
\hline date \& measured flow (cfs) & $6 / 4 / 99-182$ & $6 / 3 / 99-61$ \\
\hline date \& measured flow (cfs) & $6 / 11 / 99-70$ & $6 / 10 / 99-25$ \\
\hline date \& measured flow (cfs) & $7 / 7 / 99-21$ & $7 / 6 / 99-5$ \\
\hline
\end{tabular}

Surveying and measurement data were entered into the IFG4 hydraulic simulation model using velocity regression ("3-flow model”). We chose this hydraulic model because it has fewer assumptions than the alternative models and allows comparison of measured and simulated data.

The initial run was reviewed to compare measured and simulated water surface elevations and velocities at each of the three calibration flows. Velocity adjustment factors (VAFs) were also reviewed; VAFs were considered acceptable if they were between 0.80 and 1.20. The initial run was also used to simulate 2.5 times the high calibration flow and 0.4 times the low calibration flow. These upper and lower extrapolations were reviewed for extremely high and unlikely velocities or velocity distributions, as well as patterns of water surface elevation from transect to transect and VAFs. 
In comparisons of measured and simulated velocities, I tallied "good" matches and "poor" matches. A match between measured and simulated velocity was considered "good" if the simulated velocity was within $0.20 \mathrm{fps}$ or between $80 \%$ and $120 \%$ of the measured velocity at a point. Otherwise it was considered a "poor" match. This standard is based on the assumption that at velocities less than $1.0 \mathrm{fps}$ some error is acceptable without biological consequences: water is still relatively slow if it is 0.4 instead of $0.2 \mathrm{fps}$, but a $20 \%$ tolerance would allow almost no error at low velocity. At high velocities, a 20\% error can be substantial, e.g., $6 \mathrm{fps}$ instead of $5 \mathrm{fps}$, but both velocities are high for most fish.

Each "poor" velocity was reviewed along with the pattern for the other two measurements at that measurement point or vertical. Input velocities were modified from measured velocities where such changes were expected to improve the match at all three flows between measured and simulated velocities. Subsequent calibration runs were compared for the numbers of "good" and "poor" matches. A new calibration run was only accepted if the number of "good" matches at each transect was no less than the number in the previous calibration run. The upper and lower extrapolations were also reviewed at each calibration to determine if extrapolated velocities were reasonable; any simulated velocity over $10.0 \mathrm{fps}$ was considered unreasonable.

Calibration criteria are compared between initial and final calibration runs in Tables 2 and 3.

Walla Walla River

Water surface elevations (stage) are modeled reasonably well at the Walla Walla River below Mill Creek (Table 2). At the 3 calibration flows the simulated water surface elevations are within $0.05 \mathrm{ft}$ except for transect 9 . At transect 9 , water surface was underestimated by 0.06 feet at the high calibration flow ( $182 \mathrm{cfs}$ ), overestimated by $0.13 \mathrm{ft}$ at $70 \mathrm{cfs}$, and underestimated by $0.07 \mathrm{ft}$ at the low calibration flow $(21 \mathrm{cfs})$. All simulated water surface elevations increase in an upstream direction except at the low simulated flow $(10 \mathrm{cfs})$, where transect 2 is $0.07 \mathrm{ft}$ lower than the transect downstream (\#1) and transect 8 is 0.01 lower than transect 7. Transect 9 was the head of a pool at higher flows, but at low flow it included elevated riffle and pool.

Table 2. Walla Walla River water surface elevations: measured/simulated - run 5

\begin{tabular}{|c|c|c|c|c|c|c|c|c|}
\hline Transect & $450 \mathrm{cfs}$ & & $\mathrm{cfs}$ & & $\mathrm{cfs}$ & & $1 \mathrm{cfs}$ & $10 \mathrm{cfs}$ \\
\hline & meas simulated & leas & simulated & eas & simulated & meas & simulated meas & simulated \\
\hline 1 & 98.5 & 97.93 & 97.9 & 97.39 & 97.43 & 97.01 & 97 & 96.9 \\
\hline 2 & 98.74 & 98.1 & 98.07 & 97.48 & 97.53 & 97.07 & 97.05 & 96.83 \\
\hline 3 & 99.71 & 99.13 & 99.11 & 98.64 & 98.66 & 98.29 & 98.28 & 98.12 \\
\hline 4 & 100.18 & 99.56 & 99.53 & 98.99 & 99.03 & 98.6 & 98.59 & 98.39 \\
\hline 5 & 100.27 & 99.64 & 99.62 & 99.07 & 99.1 & 98.66 & 98.65 & 98.44 \\
\hline 6 & 100.66 & 99.92 & 99.88 & 99.25 & 99.3 & 98.84 & 98.82 & 98.62 \\
\hline 7 & 101.96 & 101.21 & 101.18 & 100.52 & 100.57 & 100.04 & 100.02 & 99.78 \\
\hline 8 & 102.16 & 101.31 & 101.29 & 100.58 & 100.61 & 100.04 & 100.03 & 99.77 \\
\hline 9 & 101.82 & 101.31 & 101.25 & 100.66 & 100.79 & 10.43 & 100.36 & 100.16 \\
\hline
\end{tabular}

Simulated velocities more closely matched measured velocities at the three calibration flows at the final simulation compared to the initial simulation (Table 3).

Table 3. Number of velocities within $20 \%$ and/or $0.20 \mathrm{fps}$ of measured velocity

- Walla Walla

Transect

initial

$130 / 32$

$182 \mathrm{cfs}$

$70 \mathrm{cfs}$

final initial

final

$21 \mathrm{cfs}$

$225 / 30$

$30 / 32$

$28 / 31$

$30 / 31$

initial final

$331 / 39$

$30 / 30$

$19 / 26$

$21 / 26$

23/25

$27 / 27$

$435 / 38$

$30 / 39$

$11 / 32$

$14 / 32$

$7 / 13$

$19 / 21$

$544 / 55$

$38 / 38$

29/30

$31 / 32$

$21 / 27$

$21 / 25$

$22 / 24 \quad 22 / 24$

$630 / 37$

$48 / 48$

$36 / 37$

$45 / 45$

$30 / 32$

$30 / 32$

$735 / 59$

$24 / 37$

$33 / 41$

$33 / 35$

$36 / 36$

$830 / 40$

$35 / 55$

$39 / 43$

$43 / 48$

$18 / 20$

$28 / 32$

$11 / 13 \quad 21 / 21$

Assessment of Salmonids and Their Habitat Conditions in 


$\begin{array}{llllll}954 / 59 & 48 / 48 & 53 / 58 & 39 / 44 & 40 / 43 & 42 / 46\end{array}$

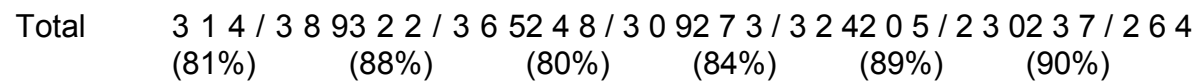

Velocity adjustment factors (VAFs) improved from the initial calibration run to the final calibration run (Table 4). However, based on the acceptable limits for VAFs, extrapolation of the model should be limited to 10-300 cfs.

Table 4. Walla Walla River velocity adjustment factors (VAFs) are quality indicators. VAFs should be between 0.80 and 1.20 .

\begin{tabular}{|c|c|c|c|c|c|c|c|c|c|c|}
\hline flow: & 10 & & 21 & & 70 & & 182 & & 450 & \\
\hline transect & initial & final & initial & final & initial & final & initial & final & initial & final \\
\hline $\mathrm{T} 1$ & 1.04 & 1.04 & 1.00 & 1.01 & 1.01 & 1.00 & .99 & .99 & .76 & .83 \\
\hline $\mathrm{T} 2$ & .87 & .88 & .98 & .96 & 1.08 & 1.06 & .97 & .97 & .40 & .60 \\
\hline $\mathrm{T} 3$ & 1.15 & 1.09 & 1.09 & 1.03 & 1.01 & .97 & .81 & .96 & .00 & .96 \\
\hline $\mathrm{T} 4$ & .97 & .97 & .97 & .97 & .99 & .99 & 1.00 & .99 & .67 & .91 \\
\hline T5 & .95 & .95 & .99 & .99 & 1.01 & 1.01 & .98 & .99 & .90 & .94 \\
\hline T6 & 1.06 & 1.07 & 1.01 & 1.02 & .96 & .99 & .93 & .99 & .87 & .95 \\
\hline $\mathrm{T} 7$ & .95 & .95 & .99 & .98 & 1.02 & 1.01 & .97 & .98 & .86 & .92 \\
\hline T8 & 1.21 & 1.19 & 1.09 & 1.00 & 1.03 & 1.02 & 1.02 & 1.03 & .93 & .93 \\
\hline T9 & 1.00 & .99 & 1.03 & 1.02 & 1.02 & .99 & .91 & .97 & .60 & .88 \\
\hline
\end{tabular}

\section{Mill Creek}

Water surface elevations (stage) are modeled reasonably well at Mill Creek (Table 5). At the 3 calibration flows the simulated water surface elevations are within $0.07 \mathrm{ft}$. All simulated water surface elevations increase in an upstream direction except transect 1 , which is $0.10 \mathrm{ft}$ higher than the next transect upstream (\#2) at the high simulated flow (135 cfs); 0.02 and $0.05 \mathrm{ft}$ at the high simulated and measured calibration flow (56 cfs), respectively; 0.015 and $0.02 \mathrm{ft}$ at the medium simulated and measured calibration flow ((22 cfs), respectively. These deviations from the expected pattern include surveyed water surface elevations at higher flows, and the differences are not considered a problem at the range of extrapolations.

Table 5. Mill Creek water surface elevations: measured/simulated - run 5

\begin{tabular}{|c|c|c|c|c|c|c|c|c|c|c|c|c|}
\hline Transec & 13 & $5 \mathrm{cfs}$ & $56 \mathrm{cf}$ & & 22 & & $6 \mathrm{cfs}$ & & $3 c$ & & & \\
\hline & meas & sim & meas & sim & meas & $\operatorname{sim}$ & meas & sim & meas & simulat & & \\
\hline 1 & & 98.87 & 98.55 & 98.55 & 98.31 & 98.31 & 98.10 & 98.10 & & 98.03 & & \\
\hline 2 & & 98.77 & 98.53 & 98.50 & 98.295 & & 98.29 & 98.11 & 98.11 & & 98.04 & \\
\hline 3 & & 99.13 & 98.88 & 98.86 & 98.605 & & 98.63 & 98.41 & 98.40 & & 98.30 & \\
\hline 4 & & 99.55 & 99.415 & & 99.39 & 99.195 & & 99.26 & 99.03 & 99.10 & & 99.04 \\
\hline 5 & & 99.84 & 99.635 & & 99.60 & 99.37 & 99.40 & 99.21 & 99.19 & & 99.10 & \\
\hline 6 & 100.04 & 100.72 & & $\begin{array}{c}100.50 \\
99.15\end{array}$ & & 100.47 & & 100.20 & & 100.26 & & 100.07 \\
\hline 7 & 101.41 & 101.79 & & $\begin{array}{l}101.69 \\
101.33\end{array}$ & 5101.68 & & 101.52 & & 101.56 & & 101.43 & \\
\hline 8 & 102.76 & 103.71 & & $\begin{array}{l}103.36 \\
102.63\end{array}$ & & 103.36 & & 103.07 & & 103.07 & & 102.76 \\
\hline
\end{tabular}

Simulated velocities more closely matched measured velocities at the three calibration flows at the final simulation compared to the initial simulation (Table 6).

Table 6. Number of velocities within $20 \%$ and/or 0.20 fps of measured velocity - Mill Creek 


\begin{tabular}{lllllll}
\multicolumn{2}{c}{ Transect } & $56 \mathrm{cfs}$ & \multicolumn{2}{c}{$22 \mathrm{cfs}$} & \multicolumn{2}{c}{$6 \mathrm{cfs}$} \\
& initial & final & initial & final & initial & final \\
1 & $22 / 26$ & $23 / 26$ & $21 / 26$ & $25 / 26$ & $24 / 26$ & $24 / 26$ \\
2 & $18 / 22$ & $19 / 22$ & $19 / 21$ & $18 / 21$ & $16 / 20$ & $18 / 20$ \\
3 & $22 / 27$ & $23 / 27$ & $15 / 25$ & $20 / 25$ & $20 / 21$ & $19 / 21$ \\
4 & $29 / 29$ & $28 / 29$ & $24 / 29$ & $23 / 29$ & $25 / 27$ & $25 / 27$ \\
5 & $25 / 28$ & $26 / 28$ & $18 / 25$ & $22 / 25$ & $19 / 21$ & $20 / 21$ \\
6 & $25 / 28$ & $24 / 28$ & $20 / 27$ & $21 / 27$ & $22 / 23$ & $23 / 23$ \\
7 & $29 / 32$ & $28 / 32$ & $21 / 25$ & $21 / 25$ & $13 / 14$ & $14 / 14$ \\
8 & $34 / 35$ & $35 / 35$ & $33 / 34$ & $32 / 34$ & $30 / 30$ & $30 / 30$ \\
Total & $169 / 182$ & $173 / 182$ & $176 / 202$ & $182 / 202$ & $204 / 227$ & $206 / 227$ \\
& $(90 \%)$ & $(95 \%)$ & $(85 \%)$ & $(90 \%)$ & $(93 \%)$ & $(91 \%)$
\end{tabular}

Velocity adjustment factors improved from the initial calibration run to the final calibration run (Table 7). However, the range of extrapolation for the Mill Creek model should be limited to 5-110 cfs, based on an acceptable range of 0.8 to 1.2.

Table 7. Mill Creek velocity adjustment factors (VAFs) are quality indicators. VAFs should be between 0.80 and 1.20 .

\begin{tabular}{lllllllllll} 
flow: & & $135 \mathrm{cfs}$ & $56 \mathrm{cfs}$ & \multicolumn{3}{c}{$22 \mathrm{cfs}$} & \multicolumn{7}{c}{$3 \mathrm{cfs}$} \\
transect & initial & final & initial & final & initial & final & initial & final & initial & final \\
T1 & 0.53 & 0.74 & 0.91 & 0.97 & 1.10 & 1.02 & 1.01 & 0.89 & 0.86 & 0.78 \\
T2 & 0.85 & 0.86 & 0.99 & 0.98 & 1.07 & 1.06 & 1.19 & 1.00 & 1.29 & 0.87 \\
T3 & 0.54 & 0.83 & 0.96 & 0.98 & 1.07 & 1.03 & 0.97 & 0.95 & 0.88 & 0.85 \\
T4 & 0.79 & 0.80 & 0.92 & 0.91 & 1.00 & 1.01 & 1.09 & 1.08 & 1.13 & 1.13 \\
T5 & 0.80 & 0.92 & 0.96 & 0.99 & 1.01 & 1.01 & 0.98 & 0.98 & 0.94 & 0.95 \\
T6 & 0.67 & 0.90 & 0.94 & 0.95 & 0.99 & 0.99 & 1.02 & 1.02 & 1.04 & 1.04 \\
T7 & 0.86 & 0.86 & 0.98 & 0.97 & 0.97 & 0.96 & 0.82 & 0.83 & 0.72 & 0.72 \\
T8 & 0.63 & 0.67 & 0.99 & 0.98 & 1.01 & 1.01 & 0.98 & 0.99 & 0.97 & 0.97
\end{tabular}

Results

Walla Walla River below mouth of Mill Creek

Habitat (WUA) for juvenile steelhead, the primary species and life-stage of interest at this site, increased consistently from the lowest simulated flow of $10 \mathrm{cfs}$ through the lowest measured flow $(21 \mathrm{cfs})$ through the highest measured $(182 \mathrm{cfs})$ and extrapolated (300 $\mathrm{cfs})$ flows (Tables 8 and 9). It should not be assumed that $300 \mathrm{cfs}$ maximizes juvenile steelhead WUA. Juvenile steelhead WUA increases most rapidly up to $100 \mathrm{cfs}$ (Table 10). WUA for juvenile steelhead at $100 \mathrm{cfs}$ is about $80 \%$ of the WUA at $300 \mathrm{cfs}$ (Table 9).

Although this reach of the Walla Walla River is not prime steelhead spawning habitat, we modeled steelhead spawning habitat in case future water and land management makes it a more suitable reach for steelhead spawning. Steelhead spawning WUA at this site peaks at $182 \mathrm{cfs}$, our high calibration flow (Tables 8 and 9).

Chinook salmon were extirpated from the Walla Walla River drainage about 1950, but some efforts are aimed at restoration of chinook runs in the Walla Walla. We modeled juvenile chinook salmon rearing habitat to provide information for use in chinook restoration. We do not anticipate any spring chinook spawning in this reach because of high temperatures and poor holding conditions, so we have not modeled chinook spawning habitat. Habitat (WUA) for juvenile chinook increased consistently from the lowest simulated flow of $10 \mathrm{cfs}$ through the lowest measured flow $(21 \mathrm{cfs})$ to the middle measured flow ( $70 \mathrm{cfs})$, declined slightly to $125 \mathrm{cfs}$, then increased through the highest measured (182 cfs) and extrapolated (300 cfs) flows (Tables 8 and 9 ). It should not be assumed that $300 \mathrm{cfs}$ maximizes juvenile chinook WUA. Juvenile chinook WUA increases most rapidly up to $30-50 \mathrm{cfs}$ (Table 10). WUA for juvenile chinook at $30 \mathrm{cfs}$ is about $71 \%$ and at $50 \mathrm{cfs}$ about $84 \%$ of the WUA at $300 \mathrm{cfs}$ (Table 9).

Table 8. Square feet of Weighted Usable Area (WUA) per thousand feet of stream in the Walla Walla River below the mouth of Mill Creek, as measured and modeled in June-July, 1999.

\begin{tabular}{|c|c|c|c|c|}
\hline Flow (cfs) & $\begin{array}{l}\text { teelhead } \\
\text { VUA }\end{array}$ & & $\begin{array}{l}\text { Chinook } \\
\text { salm on } \\
\text { WUA }\end{array}$ & $\begin{array}{l}\text { W e t t e d } \\
\text { channel }\end{array}$ \\
\hline & Ivenile & spawning & juvenile & gross area \\
\hline 10 & 224.27 & 26.65 & 667.21 & 27752 \\
\hline 21 & 495.2 & 496.34 & 1266.27 & 35020 \\
\hline 30 & 736.1 & 1360.15 & 1529.16 & 37303 \\
\hline
\end{tabular}




$\begin{array}{rrrrr}40 & 979.6 & 2861.47 & 1684.58 & 38651 \\ 50 & 1207.22 & 4779.38 & 1808.22 & 39684 \\ 60 & 1405.79 & 6468.45 & 1938.27 & 41360 \\ 70 & 1595.52 & 8142.4 & 1991.77 & 43792 \\ 80 & 1754.83 & 9674.53 & 1949.66 & 44771 \\ 100 & 1976.7 & 12362.91 & 1745.9 & 47282 \\ 125 & 2092.39 & 15086.33 & 1712.9 & 49378 \\ 150 & 2141.59 & 16780.77 & 1748.99 & 52528 \\ 182 & 2199.93 & 17197.71 & 1921.72 & 55959 \\ 200 & 2258.34 & 16748.66 & 1982.25 & 57952 \\ 250 & 2369.95 & 15007.43 & 1986.77 & 62839 \\ 300 & 2480.15 & 13341.04 & 2152.59 & 65424 \\ 350 & \text { X2612.76 X11940.86 } & \text { X2224.25 } & 68749 \\ 400 & \text { X2760.96 X10617.89 } & \text { X2333.52 } & 71629 \\ 450 & \text { X2943.85 X9879.21 } & \text { X2606.73 } & 74024\end{array}$

Table 9. Fraction of maximum modeled Weighted Usable Area at each flow of interest in the Walla Walla River below the mouth of Mill Creek.

\begin{tabular}{rrrr} 
Flow (cfs) $\begin{array}{l}\text { Steelhead } \\
\text { WUA }\end{array}$ & $\begin{array}{l}\text { Chino ok } \\
\text { s a I m o n } \\
\text { WUA }\end{array}$ \\
\multicolumn{2}{c}{ juvenile } & spawning & $\begin{array}{r}\text { juvenile } \\
10\end{array}$ \\
0.090 & 0.002 & 0.310 \\
21 & 0.200 & 0.029 & 0.588 \\
30 & 0.297 & 0.079 & 0.710 \\
40 & 0.395 & 0.166 & 0.783 \\
50 & 0.487 & 0.278 & 0.840 \\
60 & 0.567 & 0.376 & 0.900 \\
70 & 0.643 & 0.473 & 0.925 \\
80 & 0.708 & 0.563 & 0.906 \\
100 & 0.797 & 0.719 & 0.811 \\
125 & 0.844 & 0.877 & 0.796 \\
150 & 0.863 & 0.976 & 0.813 \\
182 & 0.887 & 1 & 0.893 \\
200 & 0.911 & 0.974 & 0.921 \\
250 & 0.956 & 0.873 & 0.923 \\
300 & 1 & 0.776 & 1
\end{tabular}

Table 10. Incremental rate of change of juvenile steelhead and chinook salmon WUA at each flow of interest at the Walla Walla River below Mill Creek.

Flow (cfs) juvenile steelhead juvenile chinook salmon

$\begin{array}{lll}10 & & \\ 21 & 0.0099 & 0.0253 \\ 30 & 0.0108 & 0.0136 \\ 40 & 0.0098 & 0.0072 \\ 50 & 0.0092 & 0.0057 \\ 60 & 0.0080 & 0.0060\end{array}$




$\begin{array}{rrr}70 & 0.0076 & 0.0025 \\ 80 & 0.0064 & -0.0020 \\ 100 & 0.0045 & -0.0047 \\ 125 & 0.0019 & -0.0006 \\ 150 & 0.0008 & 0.0007 \\ 182 & 0.0007 & 0.0025 \\ 200 & 0.0013 & 0.0016 \\ 250 & 0.0009 & 0.0000 \\ 300 & 0.0009 & 0.0015\end{array}$

Mill Creek

Habitat (WUA) for juvenile steelhead, the primary species and life-stage of interest at this site, increased consistently from the lowest simulated flow of $3 \mathrm{cfs}$ through the lowest measured flow ( $6 \mathrm{cfs})$ through the highest measured (56 cfs) and simulated (135 cfs) flows (Tables 11 and 12). Juvenile steelhead WUA increases most rapidly up to $20 \mathrm{cfs}$ (Table 13). WUA for juvenile steelhead at $20 \mathrm{cfs}$ is about half of the WUA at $135 \mathrm{cfs}$ (Table 12).

Steelhead spawning WUA at this site peaks at $65 \mathrm{cfs}$ (Tables 11 and 12).

Table 11. Square feet of Weighted Usable Area (WUA) per thousand feet of stream in Mill Creek, as measured and modeled in June-July, 1999. X - indicates model results are unreliable.

\begin{tabular}{|c|c|c|c|c|}
\hline Flow (cfs) & $\begin{array}{l}\text { Steelhead } \\
\text { WUA }\end{array}$ & & $\begin{array}{l}\text { Chinook } \\
\text { salm on } \\
\text { WUA }\end{array}$ & \\
\hline & uvenile & spawning & juvenile & gross area \\
\hline 3 & X 264.69 & $\times \quad 0$ & X 220.09 & 30631 \\
\hline 4 & X 288.9 & $x$ & X 262.83 & 31539 \\
\hline 5 & 311.48 & 0 & 316.35 & 32286 \\
\hline 6 & 334.21 & 0 & 412.36 & 32753 \\
\hline 8 & 386.93 & 0 & 710.77 & 33769 \\
\hline 10 & 446.36 & 7.84 & 952.95 & 34927 \\
\hline 12 & 506.42 & 22.6 & 1184.51 & 35509 \\
\hline 15 & 598.62 & 51.15 & 1415.67 & 36270 \\
\hline 18 & 692.59 & 110.94 & 1620 & 36810 \\
\hline 20 & 756.91 & 234.01 & 1712.37 & 37213 \\
\hline 22 & 816.41 & 412.68 & 1777.61 & 37517 \\
\hline 25 & 895.58 & 770.87 & 1848.03 & 38002 \\
\hline 30 & 1007.16 & 1548.34 & 1887.77 & 38784 \\
\hline 35 & 1100.4 & 2389.66 & 1913.86 & 39348 \\
\hline 40 & 1175.47 & 3297.82 & 1944.14 & 40045 \\
\hline 45 & 1232.58 & 4208.92 & 1879.43 & 40458 \\
\hline 50 & 1277.91 & 5115.74 & 1853.52 & 40820 \\
\hline 56 & 1318.2 & 6038.11 & 1868.46 & 41168 \\
\hline 60 & 1341.52 & 6494.74 & 1865.01 & 41355 \\
\hline 65 & 1361.18 & 6841.59 & 1792.83 & 41631 \\
\hline 70 & 1369.89 & 6819.24 & 1734.12 & 41817 \\
\hline 75 & 1383.15 & 6791.88 & 1690.99 & 42686 \\
\hline 80 & 1394.16 & 6747.55 & 1651.91 & 42920 \\
\hline 90 & 1412.81 & 6573.66 & 1599.22 & 43323 \\
\hline 100 & 1444.97 & 6331.55 & 1564.31 & 43653 \\
\hline 110 & 1465.55 & 6039.21 & 1554.41 & 43920 \\
\hline
\end{tabular}




$\begin{array}{llll}120 \times 1487.08 \times 5801 & \times 1503.08 & 44169 \\ 125 \times 1497.36 \times 5694.96 & \times 1500.93 & 44287 \\ 130 \times 1504.46 \times 5569.78 & \times 1492.33 & 44394 \\ 135 \times 1511.34 \times 5444.14 & \times 1484.42 & 44669\end{array}$

Chinook salmon were extirpated from the Walla Walla River drainage about 1950, but some efforts are aimed at restoration of chinook runs in the Walla Walla. We modeled juvenile chinook salmon rearing habitat to provide information for use in chinook restoration. We do not anticipate any spring chinook spawning in this reach because of high temperatures and poor holding conditions, so we have not modeled chinook spawning habitat. Habitat (WUA) for juvenile chinook at this site peaks at $65 \mathrm{cfs}$ (Tables 11 and 12). Juvenile chinook WUA increases most rapidly up to $8 \mathrm{cfs}$ (Table 13). WUA for juvenile chinook at 8 cfs is about $37 \%$ of the WUA at $65 \mathrm{cfs}$.

Table 12. Fraction of maximum modeled Weighted Usable Area at each flow of interest in Mill Creek.

\begin{tabular}{|c|c|c|c|}
\hline \multicolumn{3}{|c|}{$\begin{array}{c}\text { Flow (cfs) Steelhead } \\
\text { WUA }\end{array}$} & \multirow{3}{*}{$\begin{array}{c}\text { Chinook } \\
\text { salmon } \\
\text { WUA } \\
\text { juvenile } \\
0.163\end{array}$} \\
\hline & enile & spawning & \\
\hline 5 & 0.213 & 0 & \\
\hline 6 & 0.228 & 0 & 0.212 \\
\hline 8 & 0.264 & 0 & 0.366 \\
\hline 10 & 0.305 & 0.001 & 0.490 \\
\hline 12 & 0.346 & 0.003 & 0.609 \\
\hline 15 & 0.408 & 0.007 & 0.728 \\
\hline 18 & 0.473 & 0.016 & 0.833 \\
\hline 20 & 0.516 & 0.034 & 0.881 \\
\hline 22 & 0.557 & 0.060 & 0.914 \\
\hline 25 & 0.611 & 0.113 & 0.951 \\
\hline 30 & 0.687 & 0.226 & 0.971 \\
\hline 35 & 0.751 & 0.349 & 0.984 \\
\hline 40 & 0.802 & 0.482 & 1 \\
\hline 45 & 0.841 & 0.615 & 0.967 \\
\hline 50 & 0.872 & 0.748 & 0.953 \\
\hline 56 & 0.899 & 0.883 & 0.961 \\
\hline 60 & 0.915 & 0.949 & 0.959 \\
\hline 65 & 0.929 & 1 & 0.922 \\
\hline 70 & 0.935 & 0.997 & 0.892 \\
\hline 75 & 0.944 & 0.998 & 0.870 \\
\hline 80 & 0.951 & 0.986 & 0.850 \\
\hline 90 & 0.964 & 0.961 & 0.823 \\
\hline 100 & 0.986 & 0.925 & 0.805 \\
\hline 110 & 1 & 0.883 & 0.800 \\
\hline
\end{tabular}

Table 13. Incremental rate of change of juvenile steelhead and chinook salmon WUA at each flow of interest in Mill Creek. 


$\begin{array}{rrr}\text { Flow (cfs) } & \text { steelhead } & \text { chinook } \\ 5 & 0.0154 & 0.0275 \\ 6 & 0.0155 & 0.0494 \\ 8 & 0.0180 & 0.0767 \\ 10 & 0.0203 & 0.0623 \\ 12 & 0.0205 & 0.0596 \\ 15 & 0.0210 & 0.0396 \\ 18 & 0.0214 & 0.0350 \\ 20 & 0.0219 & 0.0238 \\ 22 & 0.0203 & 0.0168 \\ 25 & 0.0180 & 0.0121 \\ 30 & 0.0152 & 0.0041 \\ 35 & 0.0127 & 0.0027 \\ 40 & 0.0102 & 0.0031 \\ 45 & 0.0078 & -0.0067 \\ 50 & 0.0062 & -0.0027 \\ 56 & 0.0046 & 0.0013 \\ 60 & 0.0040 & -0.0004 \\ 65 & 0.0027 & -0.0074 \\ 70 & 0.0012 & -0.0060 \\ 75 & 0.0018 & -0.0044 \\ 80 & 0.0015 & -0.0040 \\ 90 & 0.0013 & -0.0027 \\ 100 & 0.0022 & -0.0018 \\ 110 & 0.0014 & -0.0005\end{array}$

\section{Discussion}

In 1999, flow at our study sites dropped to $21 \mathrm{cfs}$ in the Walla Walla River and $6 \mathrm{cfs}$ in Mill Creek by early July. The Walla Walla River flow included the Mill Creek inflow. These were not the lowest flows of 1999, according to Mendel, et al. (2000, Appendix B). At these low flows juvenile steelhead habitat was severely reduced (20-23\% of WUA at highest simulated flows). Juvenile steelhead WUA increases most rapidly up to $100 \mathrm{cfs}$ in the Walla Walla River and up to $20 \mathrm{cfs}$ in Mill Creek.

Two additional PHABSIM study sites upstream from our Walla Walla River study site are being modeled by Brad Caldwell and Jim Shedd of Washington Department of Ecology. We will combine analysis of all four sites in one report.

The Walla Walla River site below the mouth of Mill Creek is in T7N R35E S31, which corresponds to station WW-9 in the WDFW report \#FPA 00-18, Assessment of Salmonid Fishes and their Habitat Conditions in the Walla Walla River Basin of Washington: 1999 Annual Report, by Glen Mendel, David Karl, and Terrence Coyle. This site is at RM 32.9. Water temperatures at WW9 for June-November, 1999, (Appendix C, p. 65) were at or above $70 \mathrm{~F}$ during most of the summer, which are unfavorable for salmonid fishes. Flows at the site ranged from 4 cfs in early July, 1998, to no flow in early August, to $17 \mathrm{cfs}$ in mid September, and $86 \mathrm{cfs}$ in mid-November 1998, and in 1999 ranged from $15 \mathrm{cfs}$ in late June to $403 \mathrm{cfs}$ in early June (Appendix B, pp. 50). Age 0+and 1+ juvenile steelhead were observed at RM 32.9 and both upstream and downstream (Table 6, p. 28; Fig. 10, p. 30).

The report by Mendel et al. (2000) does not include sites on Mill Creek near our PHABSIM site. However, because it is upstream of WW9, it is certain to be inhabited by steelhead.

Flows modeled in this report do not ensure properly functioning habitat; they are part of what is needed for providing habitat. We report WUA, which is an index of physical microhabitat, but only if water quality is satisfactory. If temperature or dissolved oxygen or pollutants are unsatisfactory, providing abundant WUA will not ensure usable habitat for fish. Sufficient flows can contribute to favorable water quality. The flows modeled depend on channel condition and shape, including sediment distribution (gravels, sand, etc.), which in turn depend on high flows. Those high flows serve other ecological functions, including stimulation of upstream migration of adult salmonids, transport of juvenile anadromous salmonids (steelhead and salmon) to sea, and recharge of the water table. Water table recharge can contribute to riparian vegetation growth, which can shade the east-west flowing river and moderate temperature, where land use allows riparian trees to grow. 


\section{Literature Cited}

Mendel, G., D. Karl, and T. Coyle. 2000. Assessment of Salmonid Fishes and their Habitat Conditions in the Walla Walla River Basin of Washington: 1999 Annual Report. Washington Department of Fish and Wildlife, Fish Program, Fish Management Division. Report \#FPA 00-18.

cc: $\quad$ Brad Caldwell

Jim Shedd

Michelle Eames (USFWS)

Dale Bambrick (NMFS)

Carl Samuelson 